\title{
Un seul programme : Activités pour une approche pédagogique unifiée de la sexualité, du genre, du VIH et des droits humains
}

International Sexuality and HIV Curriculum Working Group

Nicole Haberland

Population Council

Deborah Rogow

Follow this and additional works at: https://knowledgecommons.popcouncil.org/departments_sbsr-pgy

Part of the Demography, Population, and Ecology Commons, Family, Life Course, and Society Commons, Gender and Sexuality Commons, International Public Health Commons, and the Medicine and Health Commons How does access to this work benefit you? Let us know!

\section{Recommended Citation}

International Sexuality and HIV Curriculum Working Group. 2011. "Un seul programme : Activités pour une approche pédagogique unifiée de la sexualité, du genre, du VIH et des droits humains," edited by Nicole Haberland and Deborah Rogow. New York: Population Council. 


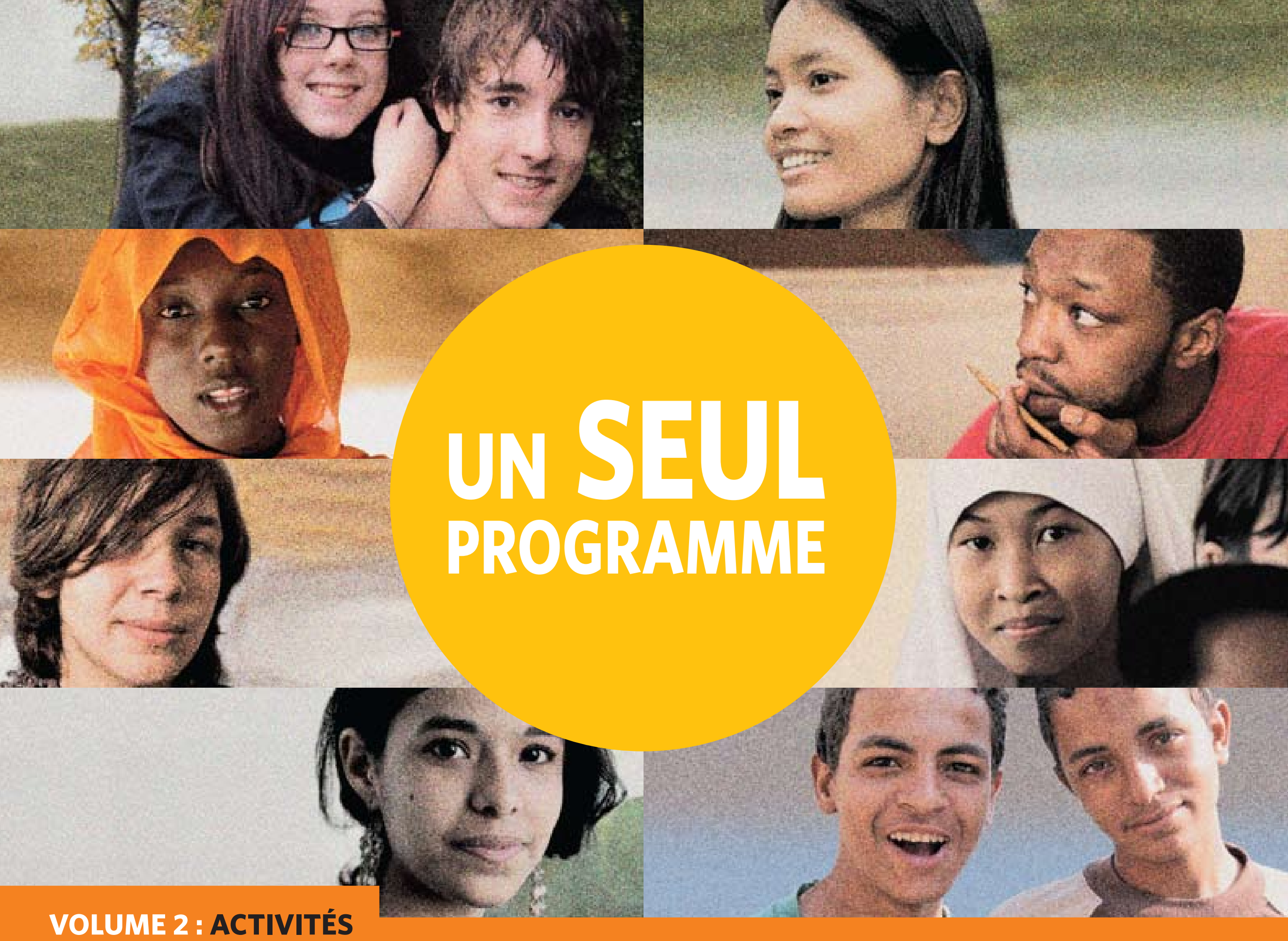

POUR UNE APPROCHE PÉDAGOGIQUE UNIFIÉE DE LA SEXUALITÉ, DU GENRE, DU VIH ET DES DROITS HUMAINS 



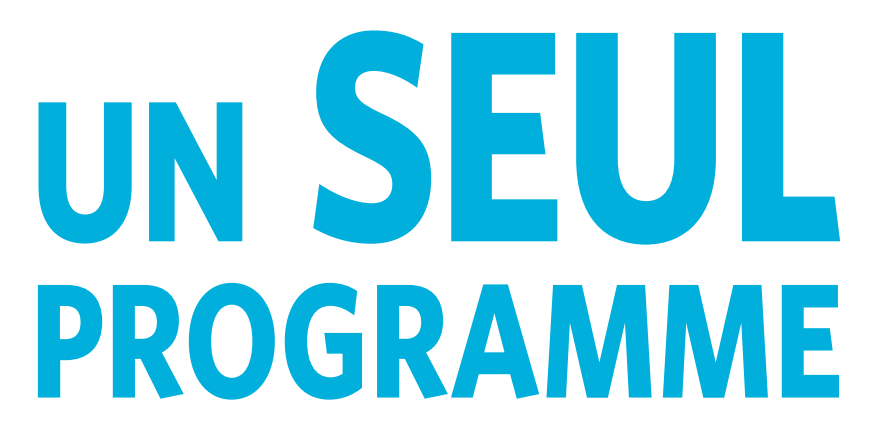

VOLUME 2 : ACTIVITÉS POUR UNE APPROCHE PÉDAGOGIQUE UNIFIÉE DE LA SEXUALITÉ, DU GENRE, DU VIH ET DES DROITS HUMAINS 

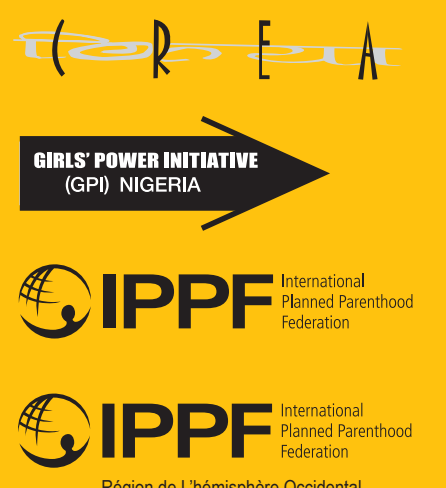

Région de L'hémisphère Occidental

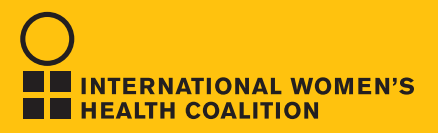

Mexfam:

(2) Population Council
Un seul programme : Guide et Activités pour une approche pédagogique unifiée de la sexualité, du genre, du VIH et des droits humains est le produit d'une initiative internationale réalisée par les représentants des organisations suivantes (par ordre alphabétique):

CREA (Inde) : Caroline Earle, Sunita Kujur, Geeta Misra

Girls Power Initiative (Nigeria) : Bene Madunagu, Grace Osakue

International Planned Parenthood Federation : Doortje Braeken

IPPF/Western Hemisphere Region : Jessie Clyde, Denise Kohn

International Women's Health Coalition : Kelly Castagnaro, Corinne Whitaker

Mexfam (Mexique) : Ofelia Aguilar

Population Council : Nicole Haberland, Deborah Rogow

L'initiative a rassemblé diverses perspectives et expertises techniques sur l'éducation des adolescents à la sexualité et prévention du VIH, dans l'espace droits et genre, de la gestion programmatique, de la recherche et du plaidoyer. Andrea Irvin a également participé à la conception de plusieurs sections. Cette traduction française a été réalisée avec le soutien de IPPF et a été révisée par Michelle Skaer.

\section{Rédaction : International Sexuality and HIV Curriculum Working Group Édition : Nicole Haberland et Deborah Rogow}

Traduction : Geneviève Haines

Coordination du projet: Michelle Skaer, Jonah Stuart Brundage, Amy Handler

Conception graphique : Emanuela Frigerio, Hyun Auh, C\&G Partners, New York City

Production : Mike Vosika, Christina Tse, Michelle Skaer, Edgar Peralta

Révision : Karen Tweedy-Holmes

Relecture : Gina Duclayan

Crédits photos des images de couverture (sens horaire, à partir du haut à gauche): Maura Carroll; Ronn Aldaman; Eugene Martin; Manoocher Ceghati, avec I'autorisation d'IRIN; Melissa May; Gabe Cooney, avec l'autorisation d'IPPF/WHR; Michael Newman; Neil Thomas, avec l'autorisation d'IRIN. Crédits photos des chapitres: Méthodes d'enseignement efficaces, Mark Tuschman, avec l'autorisation d'UNFPA Guatemala; Activités, Andrea Lynch, avec l'autorisation d'EMpower et CASA Mexico; Ressources complémentaires, Amy Joyce. Tous autres crédits identifiés en regard de l'image.

Publié par The Population Council, New York.

Téléchargement gratuit depuis: www.unseulprogramme.org

Copyright (c) 2011 The Population Council, Inc.

Toute partie de ce volume peut être photocopiée sans l'autorisation des auteurs ou de l'éditeur, sous mention expresse de la source et distribution gratuite des copies. Toute reproduction commerciale est soumise à autorisation préalable écrite du Population Council. Prière de mentionner aussi toutes sources originales indiquées pour les activités.

ISBN: Guide: 978-0-87834-124-5; Activités: 978-0-87834-125-2; Set: 978-0-87834-126-9 


\section{table des matières}

MÉTHODES D'ENSEIGNEMENT EFFICACES

ACTIVITÉS

17

DROITS HUMAINS SONT NÉCESSAIRES POUR LA SANTÉ SEXUELLE

ET LE BIEN-ÊTRE

activités 1-4

LE GENRE

activités 5-16

LA SEXUALITÉ

activités 17-26

LES RELATIONS INTERPERSONNELLES

activités $27-33$

APTITUDES À LA COMMUNICATION ET À LA DÉCISION

activités 34-39

LE CORPS, LA PUBERTÉ ET LA REPRODUCTION

activités $40-45$

LA SANTÉ SEXUELLE ET GÉNÉSIQUE

activités 46-52

PLAIDOYER POUR LA SANTÉ SEXUELLE, LES DROITS

ET L'ÉGALITÉ DE GENRE

activités 53-54

RESSOURCES COMPLÉMENTAIRES
Un seul programme est présenté en deux volumes. Le premier (GUIDE) contient une introduction, 8 unités de contenu et 22 fiches d'information.

Le second, celui-ci, est un volume

compagnon d'ACTIVITÉS. 


\section{MÉTHODES}

D'ENSEIGNE ENT

EFFICACES 


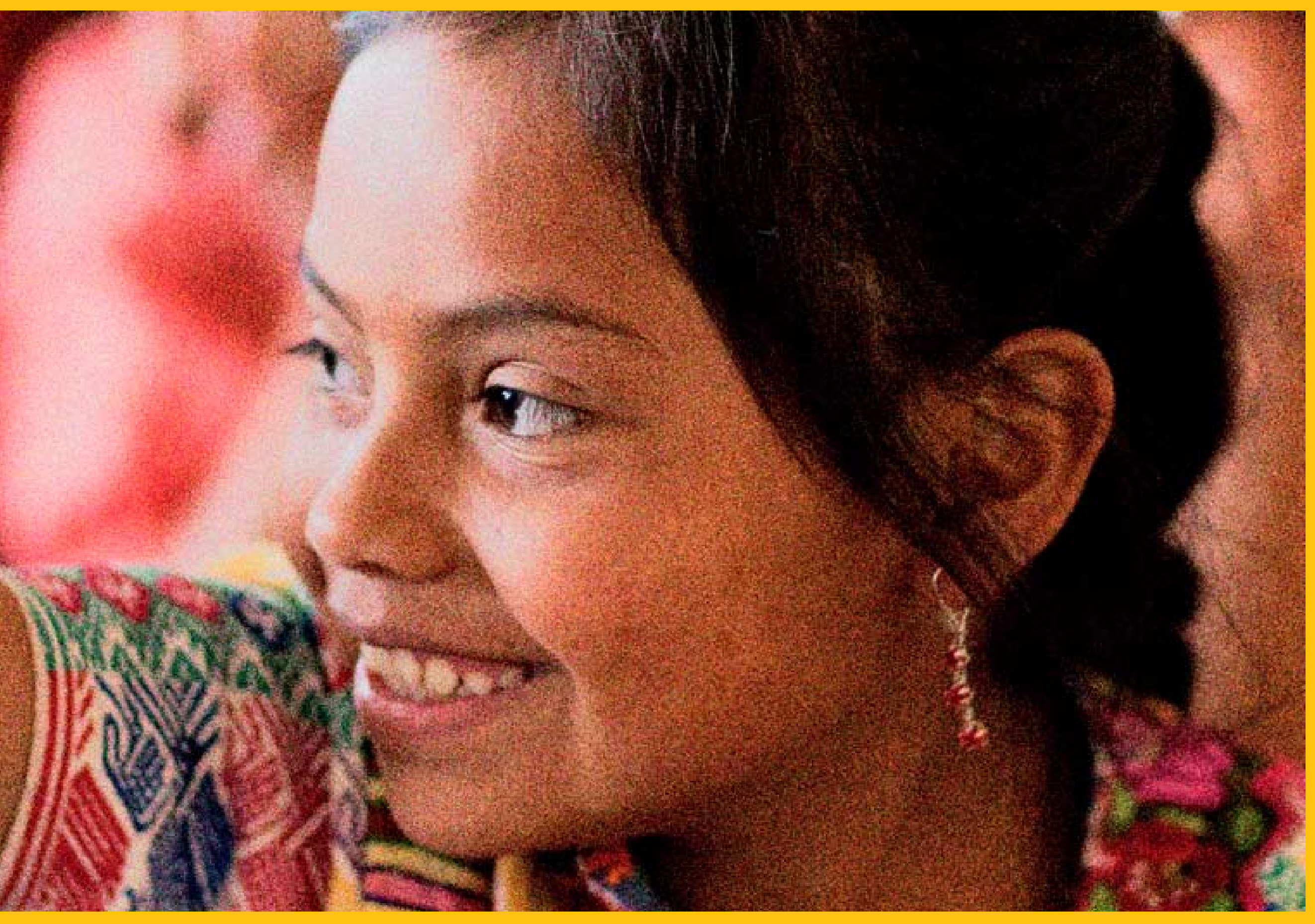




\section{remarques à l'intention des éducateurs et concepteurs de programmes d'enseignement}

Le contenu d'un enseignement est tout aussi important que son approche. Les méthodes d'enseignement les plus efficaces sur les thèmes de la sexualité, du VIH, du genre et des droits sont les méthodes participatives centrées sur l'apprenant. Ces méthodes préparent en effet les jeunes à vivre, de manière propice à leur santé et à leur bonheur, dans un monde complexe et changeant.

Pour pratiquer les méthodes participatives avec aisance et compétence, les enseignants doivent y être préparés et soutenus. Cette section présente un aperçu des principes fondamentaux de l'enseignement interactif centré sur l'apprenant, ainsi que quelques conseils pratiques d'application spécifique aux programmes sur la sexualité et le VIH. Elle propose aussi quelques directives de mise en œuvre de ces programmes dans le contexte scolaire et communautaire ${ }^{1}$.

Un seul programme est présenté en deux volumes. Celui-ci (ACTIVITÉs) propose une sélection de 54 activités de classe engageantes. Le premier volume (GUIDE) encourage aussi le recours aux méthodes participatives. Ses unités de contenu proposent notamment d'occasionnels " Points de réflexion " propices à l'analyse critique de certains sujets, tandis que l'unité 8 invite les élèves à l'expérience pratique. 


\section{derrière les méthodes d'enseignement interactives centrées sur l'apprenant}

L'apprentissage n'est pas un processus d'absorption passive. Il s'agit plutôt d'une collecte d'information, réinterprétée en fonction de la connaissance et du vécu de chaque individu. Pour optimiser ce processus, l'enseignant peut engager activement l'élève dans son propre apprentissage .

Ainsi, nombreux sont les chercheurs qui estiment que les méthodes d'enseignement interactives centrées sur l'apprenant produisent de meilleurs résultats d'apprentissage ${ }^{2}$. Elles éveillent l'intérêt de l'élève et stimulent l'analyse critique $^{3}$. En ce qui concerne surtout les thèmes qui affectent directement la vie des élèves, ces méthodes les aident à personnaliser l'information et à pratiquer de nouvelles compétences ${ }^{4}$.

Au fil du temps, les méthodes d'enseignement participatives sont devenues l'approche principale de nombreux programmes d'éducation à la sexualité et sur le VIH. De plus en plus d'enseignants recourent désormais à ces méthodes dynamiques, les complétant de projets « pratiques » appliqués à différentes branches de l'enseignement : études sociales, instruction civique, science et littérature. La participation et l'expression libre à l'école sont du reste propices aux attitudes démocratiques. Une enquête menée dans plusieurs pays révèle notamment que l'apprentissage dans ce contexte de classe ouverte favorise, parmi les élèves, une meilleure acceptation de l'égalité de genre ${ }^{5}$. Les enseignants eux-mêmes reconnaissent apprendre énormément à travers cette approche.

* Les programmes d'éducation relatifs à la sexualité et au VIH sont proposés dans les écoles, dans les centres communautaires et dans d'autres contextes. Aussi les termes « élève », « apprenant » et « participant » sont-ils utilisés de manière interchangeable dans ce document. 


\section{REMARQUES SPÉCIALES CONCERNANT L'ENSEIGNEMENT RELATIF AUX QUESTIONS DE GENRE, SEXUALITÉ, VIH ET SANTÉ SEXUELLE :}

Les élèves se sentent parfois mal à l'aise face à la nature sensible du sujet abordé ou à leur propre situation familiale. Veillez à préparer vos échanges pour éviter les souffrances inutiles.

Vos élèves vous parleront peutêtre d'une grossesse non planifiée, de leur expérience de la violence ou d'autres problèmes personnels ou familiaux. Réfléchissez à la manière dont vous pouvez les aider tout en maintenant des limites appropriées. Renseignezvous sur les programmes/services (refuge pour jeunes sans foyer, services pour filles violentées ou autres) proposés localement. Dans les cas de maltraitance ou de mise en danger, soyez conscient des règles de signalisation aux autorités.

Êtes-vous vous-même à l'aise face aux sujets traités? Ne manquez pas de demander aide et conseil.

Un seul programme repose sur les valeurs d'égalité, de respect et de droits humains. Pensez à vos valeurs personnelles ou religieuses et soyez sûr de pouvoir honorer celles du programme en ce qu'elles touchent au genre et à la sexualité.

\section{principes d'un enseignement interactif centré sur l'apprenant}

\section{1 Établir un contexte d'apprentissage basé sur l'égalité, le respect et les droits humains.}

- Créez un milieu favorable à l'apprentissage. Tous les élèves doivent se sentir impliqués, écoutés, à l'aise et à l'abri du ridicule, surtout quand ils prennent le risque d'émettre de nouvelles idées. Encouragez la participation, en particulier parmi ceux qui se sentent isolés ou intimidés. (Les raisons de ces sentiments sont multiples : elles sont liées, notamment, aux différences de pertinence sociale associées au genre, à la classe sociale ou à l'âge, à la connaissance limitée de la langue parlée à l'école, ou au handicap physique ou autre besoin spécial.) Les activités qui valorisent le respect d'autrui et l'esprit d'équipe, de même que le partage des occasions de leadership, favorisent la participation de la classe. Bien sûr, il est important aussi d'assurer la sécurité et le caractère privé de l'espace physique.

- En début de programme, invitez vos élèves à élaborer ensemble les directives, fondées sur le respect, qui guideront leur conduite en classe ${ }^{6}$.

- Priez-les de respecter la vie privée les uns des autres et rappelez-leur de ne pas divulguer ce qu'ils estiment appartenir strictement à leur vie privée. N’oubliez pas que certains élèves risquent d'en intimider ou même maltraiter d'autres après la discussion de sujets sensibles ${ }^{7}$. Assurez à vos élèves que vous-même, leur enseignant, garderez toutes les discussions confidentielles. Vous leur montrerez ainsi l'exemple du respect de la vie privée . $^{8}$

- Veillez à ce que les élèves comprennent bien qu'ils ont le droit de ne pas participer ou parler de leur expérience si l'idée les met mal à l'aise.

- Encouragez tous les élèves à partager leurs pensées. Ne jugez pas les idées émises. 
2 Favoriser un processus qui tire parti du vécu des élèves, en intégrant de nouvelles informations et idées dans ce qu'ils savent ou pensent déjà.

- N’oubliez pas que tous les participants ont déjà une certaine connaissance et un certain vécu. Écoutez et posez les questions propices à leur exposition.

- Écoutez les inquiétudes exprimées par les élèves et posez des problèmes hypothétiques qui reflètent la réalité de leur vie. Engagez-les dans la résolution de ces problèmes, en les amenant à opérer leurs propres choix et, ce faisant, à développer leurs idées.

- Encouragez vos élèves à explorer le sens concret, dans leur propre vie, d’un sujet abordé.

- Envisagez votre rôle comme celui d'un médiateur de débats sur les idées de vos élèves, plutôt que d'un professeur ex cathedra ou d'une simple source d'information. Au besoin, corrigez leurs erreurs factuelles ou aidez-les à reconnaître les commentaires qui manquent de respect.

- De temps à autre, il pourra vous être utile de partager un épisode pertinent de votre propre expérience. Veillez cependant toujours à maintenir les limites appropriées par rapport à vos élèves.

\section{Recourir à un vaste éventail de stratégies pour engager les élèves.}

- Préparez différentes méthodes et approches adaptables aux besoins d’apprentissage de vos élèves ${ }^{9}$. [Voir les méthodes d'enseignement interactives décrites aux pages 8 et 9.]

- Si une leçon ou une activité ne vous est pas familière, commencez par lire attentivement l'information la concernant et passez soigneusement en revue les objectifs poursuivis. Préparez à l'avance tout le matériel nécessaire !

- Préparez un plan de rechange qui vous permette de faire aisément face aux circonstances imprévues (temps disponible, météo ou réaction des élèves, par exemple).

- Consultez la liste de ressources complémentaires, en fin de volume, pour d'autres idées de méthodes et leçons d'enseignement expérientiel.

- Dans la mesure du possible, choisissez des activités amusantes !
REMARQUES SPÉCIALES

CONCERNANT L'ENSEIGNEMENT

RELATIF AUX QUESTIONS DE

GENRE, SEXUALITÉ, VIH ET

SANTÉ SEXUELLE :

Dans les contextes d'enseignement mixte, essayez de séparer les garçons des filles pour certaines activités ou discussions. Cette approche peut favoriser un sentiment d'entendement partagé et de franchise, avant de réunir les deux groupes pour la suite des débats. Au besoin, séparez la classe en deux dans un même espace physique. 


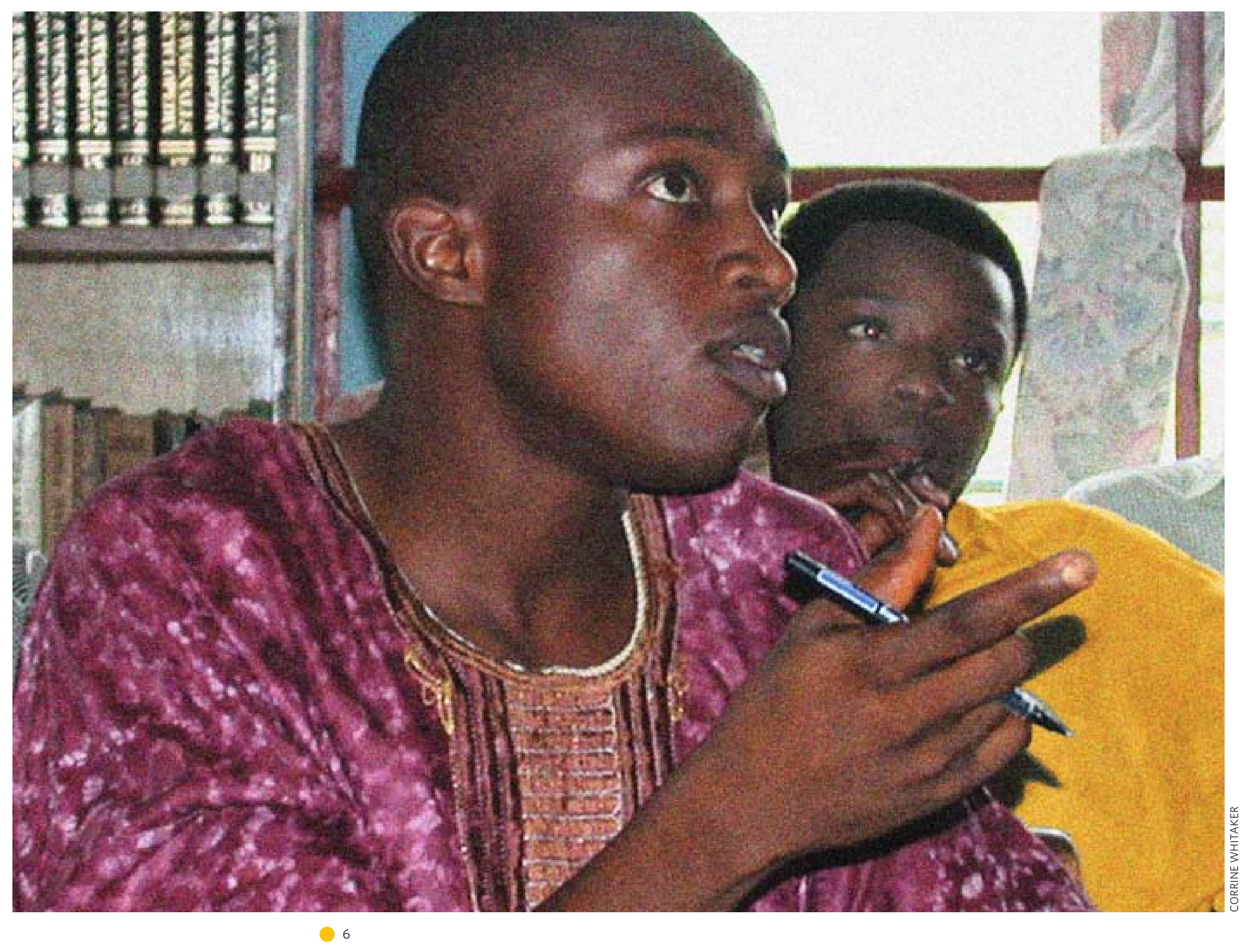


4 Renforcer les capacités de réflexion, d'étude et d'analyse critique des élèves, sur leur propre vie et sur le monde qui les entoure, ainsi que de résolution des problèmes rencontrés.

- Encouragez les élèves à remettre en question la sagesse conventionnelle. Demandez-leur de réfléchir à leurs croyances et aux normes de leur communauté. Invitez-les à explorer, respectueusement, d'autres opinions.

- Commencez chaque échange par une question ouverte. Appliquez la méthode de la maïeutique, en posant la question «pourquoi ? » pour aider vos élèves à explorer leurs contradictions et à découvrir de plus profondes vérités. Les unités 1 à 7 du volume GUIDE proposent des «points de réflexion » dont les questions encouragent spécifiquement l'analyse critique et la discussion.

- Encouragez la créativité.

- Encouragez les élèves à prendre des risques dans leur réflexion et à ne pas craindre l'erreur. Soyez prêt à démontrer vous-même cette approche.

- Organisez la classe en petits groupes d'exploration thématique et d'activité collective.

- Encouragez l'analyse des problèmes et l'apport de solutions possibles sous différents points de vue.

\section{Encourager les élèves à appliquer ce qu'ils ont appris dans leur vie et dans leur} communauté pour les aider, en somme, à devenir des citoyens actifs et la force d'un changement positif.

- Sélectionnez une matière pertinente et des méthodes d'enseignement qui invitent la réflexion critique. Invitez constamment vos élèves à mettre la matière en rapport avec leur propre vie et le monde dans lequel ils évoluent. Encouragez-les à considérer la matière à la lumière des principes d'équité et de justice sociale.

- Proposez des occasions d'apprentissage qui impliquent une recherche et une action dans le domaine des services ou dans la communauté. Veillez cependant à considérer la culture environnante et évitez de mettre vos élèves en danger.

- Considérez si et comment vous devrez défendre, au sein de votre établissement (et peut-être aussi de votre communauté), la capacité et le droit des jeunes de parler de leurs idées et de les mettre en pratique.

- N’oubliez pas que les jeunes s'inspirent souvent de leurs enseignants et des leaders de leur communauté.

\section{REMARQUES SPÉCIALES}

CONCERNANT L'ENSEIGNEMENT RELATIF AUX QUESTIONS DE GENRE, SEXUALITÉ, VIH ET SANTÉ SEXUELLE :

Soyez sensible au fait que les élèves repensent peut-être les idées reçues et jusque là admises. Aidez-les à trouver l'équilibre entre leurs idéaux et leurs ambitions, d'une part, et le maintien de la stabilité et de leur sécurité dans leur foyer et leur communauté, d'autre part.

Peut-être rencontrerez-vous une certaine résistance à l'enseignement des sujets abordés. Identifiez vos alliés au sein de votre organisation et de la communauté. Sachez aussi d'où pourra venir l'opposition à vos efforts. Recherchez appui et conseils. 


\section{MÉTHODES D'ENSEIGNEMENT INTERACTIVES APTES À ENRICHIR UN PROGRAMME}

La diversité des méthodes utilisées est la clé de l'engagement d'élèves sinon très différents les uns des autres. Elle aide aussi les élèves à établir les liens entre les thèmes abordés. Quelques méthodes propices à l'éveil de l'intérêt des élèves sont décrites ici, avec quelques conseils pratiques élémentaires.

Stimulants - Brèves activités de groupe propices à l'éveil de sentiments positifs à l'égard du groupe. Les stimulants concentrent l'énergie du groupe et peuvent servir à l'introduction de nouvelles idées. Ils favorisent la réflexion créative et sont particulièrement utiles en début de session ou pour changer le cap d'une conversation. Par exemple : jeux destinés à briser la glace, chants et exercices physiques.

Déclencheurs de discussion - Activités servant à soulever des problèmes et formuler les questions d'exploration. Ces activités sont généralement les plus efficaces en début de leçon. Par exemple : brainstorming, études de cas, questions ou déclarations invitant une réponse écrite, pour ou contre, définition d'un problème, présentation d'un petit film ou brève lecture.

Jeu créatif - Activités créatives de motivation. Ces activités stimulent la réflexion et les nouvelles idées. Elles permettent de plus aux élèves qui apprennent et communiquent le mieux sous ce format de participer utilement. Par exemple : jeux, projets artistiques, jeux de rôle, art dramatique, poésie et création littéraire. 
Discussions de groupe - Occasion pour les élèves de s'exprimer, d'être entendus et d'entendre les autres. Ces discussions les aident à développer leurs qualités d'écoute et d'expression, tout en raffinant leur réflexion et en élargissant leurs idées et leurs connaissances. Elles permettent de donner à chacun l'occasion de participer et elles favorisent l'établissement d'une culture et de valeurs démocratiques. Par exemple : dialogues informels, tribunes et débats.

Réflexion participative et analyse - Division de la classe en groupes de travail pour la résolution de problèmes. Ces activités favorisent l'unité du groupe et la réflexion critique. Par exemple : " arborescences », projets de recherche et analyse de messages médiatiques.

Réflexion personnelle - Activités utiles à la réflexion profonde des élèves sur leurs propres expériences. Ces activités renforcent la maturité et le jugement. Elles peuvent aussi ouvrir la voie à de nouvelles attitudes et de nouveaux comportements. Par exemple : tenue d'un journal, souvenirs guidés et projets d'art.

Autres outils d'enseignement participatif — Définition d'objectifs de groupe et individuels, affectation de rôles de participation à la gestion de la classe (rapporteur du jour, lecteur de citations, chronométreur, leader du jour) et révision de chaque leçon et de la matière apprise. 


\section{considérations spéciales d'élaboration et mise en œuvre d'un programme}

Un seul programme s'adresse à un public mondial. L'approche repose sur des principes universels. Inspirez-vous-en pour développer un programme ou une unité d'étude efficace adaptée à votre situation ou population locale, en prêtant attention aux facteurs pédagogiques et programmatiques de compétences des enseignants, niveau des élèves, acceptation des parents, valeurs communautaires et temps de classe disponible. Les questions ci-dessous vous guideront dans votre considération de ces facteurs. Certaines relèvent peut-être d'autres autorités : le personnel de l'organisation responsable de la mise en ouvre, par exemple, ou les partisans du programme au sein de la communauté. Elles n'en sont pas moins reprises ici car leur considération pourrait bien être la clé d'un programme réussi sur la sexualité ou la prévention du VIH. (Des références et liens à une documentation plus détaillée sur ces questions sont proposés dans les Ressources complémentaires.)

\section{Considérations d'ordre politique}

Quelles sont les politiques et ressources nationales et locales susceptibles d'affecter le succès de l'établissement du programme? Quelles sont notamment les lois ou les politiques qui régissent l'éducation relative à la sexualité et/ou à la prévention du VIH dans les écoles ? Comment y assure-t-on l'accès des jeunes handicapés ? Les responsables du programme sont-ils au courant de la recherche qui établit le lien entre les grossesses d'adolescentes, les taux d'IST, l'égalité de genre et les droits humains ? Ont-ils tenu compte de ces liens dans la conception du programme ? Votre programme ou votre établissement scolaire pourra-t-il bénéficier de sources de financement diverses ? Vous serait-il utile de cibler vos efforts de plaidoyer sur les décideurs clés de la communauté, le personnel de votre organisation ou autre? Qui sont vos alliés? 


\section{Considérations de planification}

Le programme s'intègre-t-il dans les objectifs pédagogiques plus larges de la communauté ? Quelles sont les principales questions de planification? Affecteront-elles le succès de la mise en œuvre du programme ? Quelles mesures de préparation ont été (ou doivent être) prises ? Comment assurer, par exemple, que les membres du personnel et vos partenaires clés comprennent bien l’objectif du programme et sont résolus à le voir réussir? Comment les organisateurs du programme peuvent-ils obtenir et renforcer l'appui des parents et de la communauté ? Quelles sont les ressources disponibles pour aider les parents à communiquer efficacement avec leurs enfants sur les questions de santé sexuelle? Comment profiter de l'expérience d'efforts similaires ? Comment identifier et former les enseignants et autres personnels? Quels sont les meilleurs moyens d'établir le contact avec les services de santé, de conseil et autres existants? Quels seraient les membres potentiels d'un comité directeur ou d'un groupe de travail participatif à l'élaboration du programme ? L'apport de membres de la communauté, de jeunes, de parents, de représentants du corps enseignant et de responsables des établissements scolaires et des ONG, notamment, pourrait être utile. Ce processus vous aidera à identifier et rassembler les ressources disponibles. Il renforce le programme et l'adapte davantage au contexte local, tout en donnant aux membres clés de la communauté un sentiment d'intérêt direct. Quelles sont les occasions de communication et de sensibilisation des membres de la communauté à l'égard du programme? Quelles sont les meilleures approches d'appel d'idées durant les premières phases de développement du programme ? Pensez, par exemple, aux réunions individuelles avec les leaders, à la formation de groupes de discussion, ou à la conduite d'enquêtes auprès des parents et des enseignants. Comment se préparer à faire face aux questions et inquiétudes de la communauté ?

\section{Élaboration d'un programme adapté aux situations extrêmement limitées en} ressources

Quelles sont les ressources disponibles dans le contexte considéré ? Comment adapter le contenu pour tenir compte de l'interaction entre la pauvreté et le genre, les droits, la sexualité et la santé sexuelle ? Comment adapter le programme quand les ressources sont faibles? Peut-on, par exemple, le limiter aux stratégies d'enseignement oral ou en espace extérieur ? Est-il possible d'obtenir une aide en nature de la part des ONG ou des membres de la communauté?

\section{FAIT}

L'attention aux considérations de nature politique et pédagogique joue un rôle important dans l'élaboration réussie de programmes d'éducation relatifs à la sexualité et au VIH. 


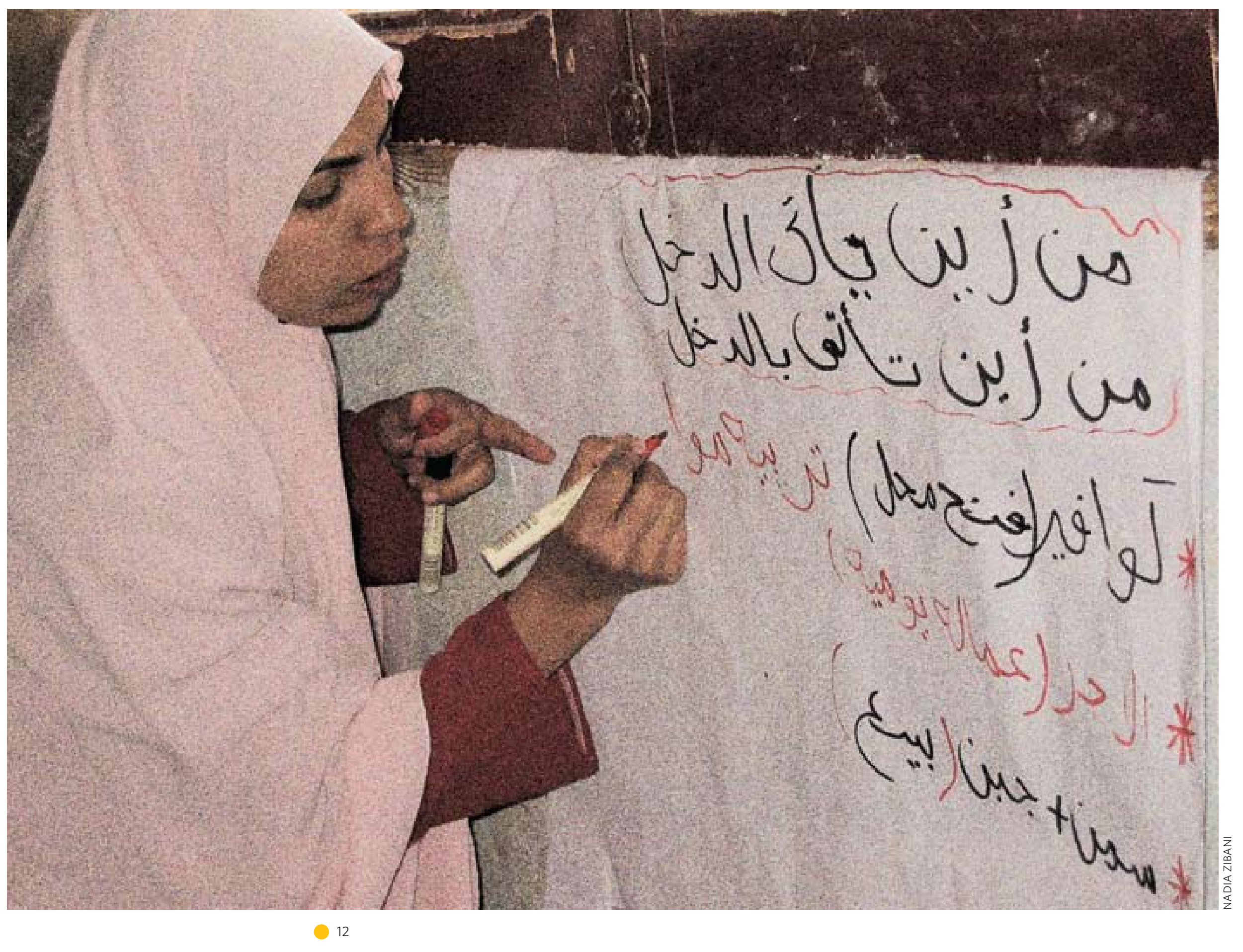




\section{Liaison du programme aux normes d'apprentissage scolaire}

Chaque activité décrite dans ce volume est associée à un objectif scolaire au sens large (exposé ou narration écrite, résolution de problème et analyse, planification et organisation d'un simple projet de recherche, ou expression verbale). Cette approche facilite l'inclusion de l'éducation relative à la sexualité et au VIH dans les matières autres que l'hygiène et la biologie, tout en précisant la manière dont ces activités peuvent aider les enseignants et les écoles à réaliser leurs objectifs pédagogiques. Durant la phase conceptuelle dans un cadre scolaire, on considérera les points suivants : quelles sont les normes d'apprentissage et indicateurs d'évaluation existants pour une matière donnée ? Compte tenu des objectifs scolaires des activités proposées, quelles sont celles qui s'inscrivent le mieux dans la poursuite de ces normes ? Quelles sont les possibilités de collaboration pour une approche interdisciplinaire de la prévention du VIH ou de la promotion de l'égalité de genre ? Plutôt que d'entrer en concurrence avec le programme général, comment une approche créative de l'éducation à la sexualité et de la sensibilisation au VIH peut-elle enrichir la qualité générale de l'enseignement?

\section{Adaptation applicable aux populations particulières}

Comment les enseignants peuvent-ils assurer la pertinence du contenu et des activités aux besoins de populations particulières? Comment modifier, par exemple, les activités pour les groupes faiblement instruits ou de l'enseignement spécial ? Peut-on assurer que le contenu reflète les besoins spéciaux de groupes tels que les jeunes travailleurs migrants, les adolescentes mariées, les soldats ou les orphelins ? Comment relever les défis pratiques de l'apprentissage de ces populations ? Pouvez-vous offrir un espace sécurisé ? Adapter les classes en fonction d'un calendrier plus limité ? Comment atteindre les élèves moins liés aux institutions de la communauté ? De quelles ressources et services d'orientation de la communauté auront-ils vraisemblablement le plus besoin ?

\section{Préparation des enseignants}

Les enseignants sont-ils prêts à adopter les approches interactives centrées sur l'apprenant? Auront-ils l'occasion d'acquérir et de développer ces compétences? Quel est le meilleur moyen d'adapter le cours à ces compétences ? Quel type de formation permettra aux enseignants d'examiner leurs propres convictions personnelles et religieuses sur la sexualité et le genre, pour mieux les préparer à enseigner ces sujets de manière conforme aux principes des droits humains ? Quelle sera la clé de l'efficacité des enseignants dans les contextes les plus conservateurs? 


\section{Évaluation}

Beaucoup d'enseignants désireront évaluer les effets de leur programme sur la sexualité et le VIH. Quels résultats ou indicateurs montreraient le mieux les progrès réalisés vers les objectifs du programme ? S'agira-t-il, par exemple, d'un changement cognitif, d'attitudes et de comportements spécifiques de la part des élèves ? Le programme amoindrira-til la contrainte et la violence dans les relations intimes? Aidera-t-il les élèves à rester à l'école ou dans un programme d'apprentissage? Accentuera-t-il la participation civique? Les nouveaux programmes prévoiront-ils une phase pilote, adaptable en fonction des premières leçons tirées?

\section{Accompagnement et dynamisme}

Vous allez créer et établir un nouveau programme d'enseignement sur la sexualité, le genre et les droits humains. Votre travail pourra susciter le scepticisme, voire l'opposition de beaucoup. Pour surmonter ces conflits, veillez à établir vos alliances dans la communauté et les institutions. Impliquez les membres clés de la communauté - y compris les parents - de manière utile avant même de commencer. Entretenez avec eux un dialogue franc et honnête. Il est essentiel que vous et vos partenaires gardiez le moral. Prenez le temps de réfléchir au programme. Progresse-t-il bien ? Atteint-il ses objectifs ? Serait-il bon de l'ajuster?

\section{Célébrez vos accomplissements et ne vous découragez surtout pas !}




\section{NOTES}

1 Ce document présente une brève introduction à la pédagogie interactive centrée sur l'apprenant. II ne prétend nullement comprendre ni remplacer un guide, atelier de formation ou une introduction formelle sur la question. Une bibliographie d'ouvrages consacrés à la question est proposée dans les Ressources complémentaires, en fin de volume.

2 Partout dans le monde, l'enseignement interactif centré sur l'apprenant s'inspire largement de la théorie de la « pédagogie critique » de Paulo Freire. Pour toutes données de résultats, en termes de réussite scolaire, attitudes des élèves, rétention et estime de soi, voir : Gross Davis, Barbara. 1993. Tools for Teaching. San Francisco: Jossey-Bass Publishers Manswell Butty, Jo-Anne. 2001. "Teacher instruction, student attitudes, and mathematics performance among 10th and 12th grade Black and Hispanic students," Journal of Negro Education 70 (1/2): 19-37; Prince, Michael. 2004. "Does active learning work? A review of the research," Journal of Engineering Education 93(3): 223-231; Slavin, Robert. 1980.

"Cooperative learning," Review of Educational Research 50(2): 315-342.

${ }^{3}$ Dans certains contextes, les éducateurs désignent l'esprit critique sous le nom de métacognition. Pour plus de détails à ce sujet et concernant I'approche dans le milieu scolaire, voir Martinez, Michael. 2006. "What is metacognition?" Phi Delta Kappan 87(9): 696-699.

4 Kirby, Doug. 2007. “Emerging Answers 2007: Research Findings on Programs to Reduce Teen Pregnancy and Sexually Transmitted Diseases." Washington, DC: National Campaign to Prevent Teen and Unplanned Pregnancy.

5 Pettersson, T. 2003. "Basic Values and Civic Education: A Comparative Analysis of Adolescent Orientations Towards Gender Equality and Good Citizenship." World Values Survey. <www.worldvaluessurvey.org/ library/>. Site consulté le 13 mai 2009.
6 Une simple introduction, où les participants définissent leurs règles de conduite et de discussion, est importante. Certains enseignants décrivent l'approche comme la « charte de la classe ». Voir, par exemple, "Making guiding rules », au chapitre 1 de chacune des trois séries Our Future de I'HIV/AIDS Alliance (programme d'éducation à la sexualité et à la dynamique de vie en Zambie, en anglais) : <www.aidsalliance.org/ custom_asp/publications/view. asp?publication_id=211\&language $=$ en $>$. Site consulté le 13 mai 2009

7 Voir, par exemple, le chapitre 1 de chacune des trois séries Our Future de l'HIV/AIDS Alliance (programme d'éducation à la sexualité et à la dynamique de vie en Zambie, en anglais) : <www.aidsalliance.org/ custom_asp/publications/view.asp?publication_id=211\&language=en> (activités « Trust and keeping secrets » et « Working together with respect »).

${ }^{8}$ Comme indiqué dans les conseils à l'enseignant de l'unité 3 du volume GUIDE, vous pouvez être tenu à une obligation légale de signalisation si un élève vous divulgue, notamment, des sévices sexuels. Informez-vous des conditions applicables à votre contexte.

9 La recherche a documenté l'immense variation entre les capacités, les styles et les besoins d'apprentissage. Par exemple, l'idée d'intelligences multiples défie la notion d'une forme unique d'intelligence (Gardner, Howard. 2006. Multiple Intelligences: New Horizons. 2nd revised edition. New York: Basic Books. <http://books.google.com/books?id=qEEC8lyAw WoC\&printsec $=$ frontcover\&source $=g b s \_g e \_s u m m a r y \_r \& c a d=0 \# v=o n e$ page\&q\&f=false $>$ ) 


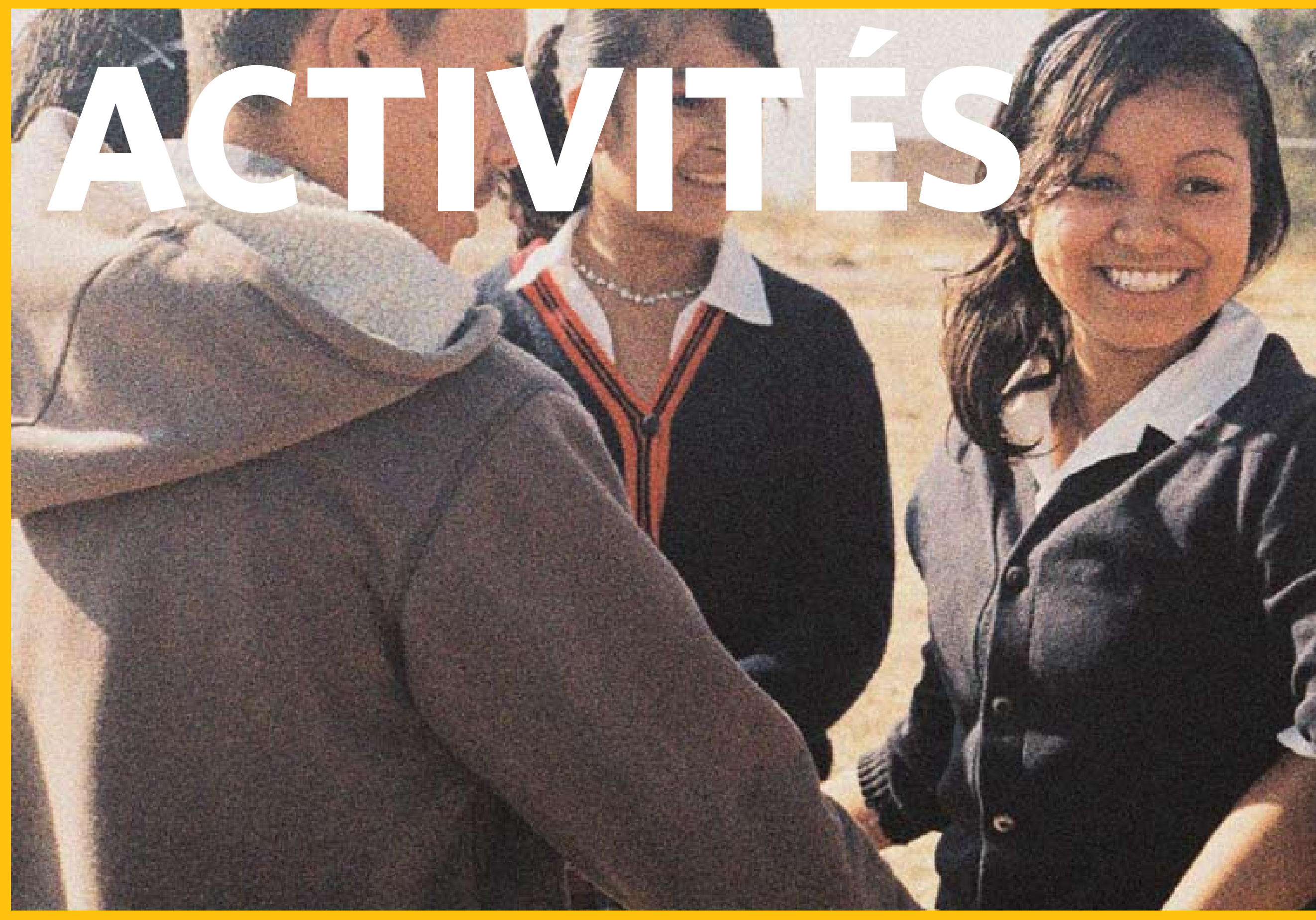




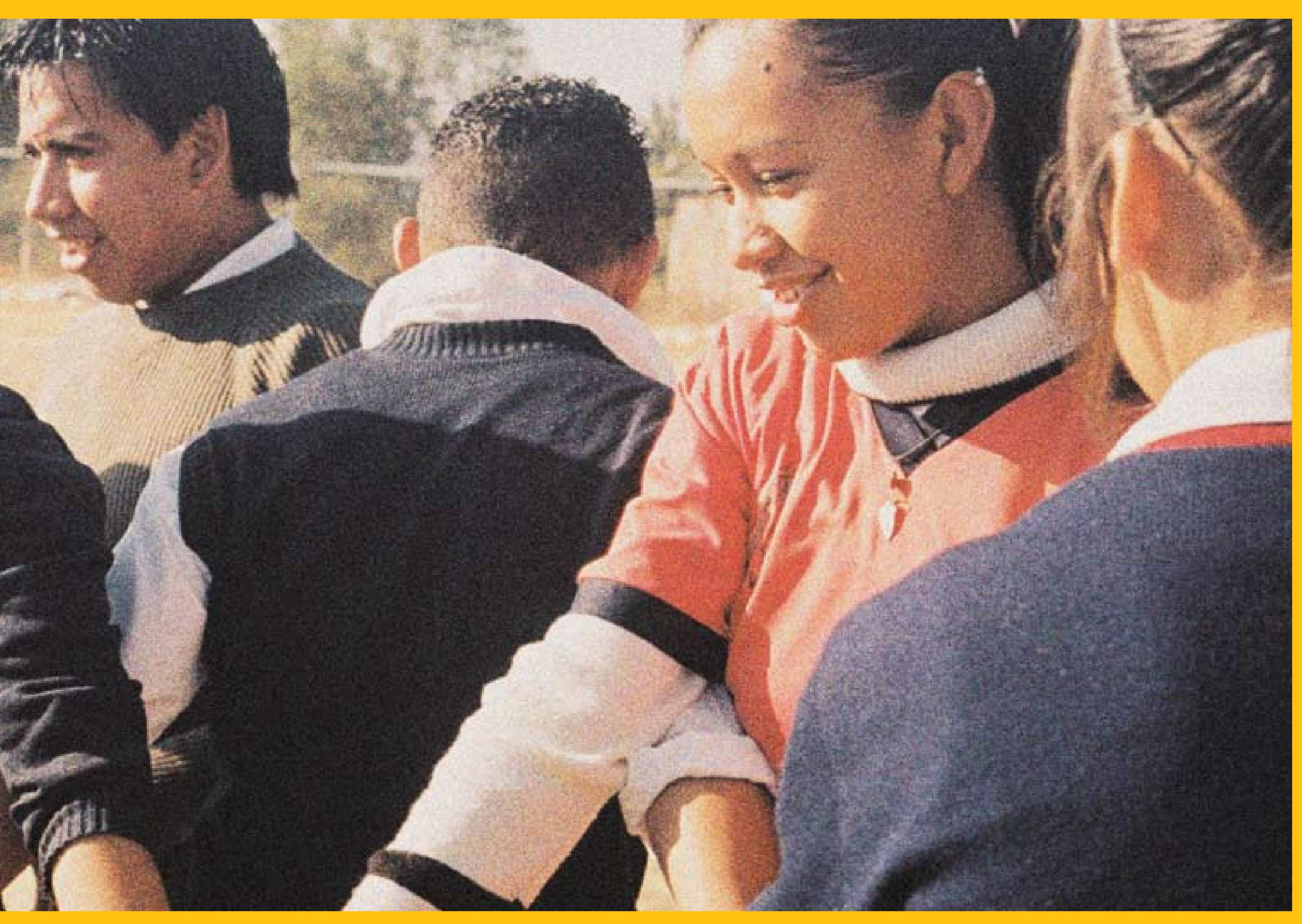




\section{bienvenue aux activités d'Un seul programme !}

\section{ÉLĖVES DE MOINS DE 15 ANS}

Cet ouvrage s'adresse principalement aux adolescents plus âgés, mais beaucoup d'activités conviennent aussi ou peuvent être adaptées, suivant le contexte, aux besoins d'enfants aussi jeunes que 10 ans.

Pour ces élèves plus jeunes, les 21 activités suivantes sont sans doute les plus utiles :

unité 1 activité 1 unité 2 activités 5-6, 8-11, 15 unité 3 activités 18, 20 unité 4 activités 27, 29, 32 unité 5 activité 36 unité 6 activités 40-43 unité 7 activités 49-50 unité 8 activité 53

\section{Ces activités ont été conçues dans un esprit pratique et d'efficacité.}

Chacune vise un objectif d'apprentissage global car, après tout, l'éducation sexuelle et VIH peut et devrait renforcer la réussite scolaire globale.

Dans cette optique, les activités s'organisent autour de deux objectifs d'apprentissage : (1) l'apprentissage relatif au thème en soi et (2) le renforcement d'aptitudes spécifiques telles que la réflexion critique, l'expression écrite, la recherche, l'art de s'exprimer en public et la planification et résolution coopératives. Comme ces activités sont de plus concrètes et souvent amusantes, elles peuvent favoriser aussi l'engagement et la connexité des élèves à l'école.

\section{Chaque activité est liée à un thème spécifique du GUIDE.}

Plusieurs impliquent notamment le recours aux fiches d'information du GUIDE, accessible en ligne sur $<$ www.unseulprogramme.org $>$.

La série complète d'activités s'adresse aux jeunes de 15 ans et plus. Beaucoup d'activités individuelles conviennent cependant aussi aux enfants plus jeunes.

Voir ci-contre (marge gauche) la liste des activités également appropriées pour les jeunes de 10 à 14 ans.

Les activités prévoient toutes les feuilles d'accompagnement et feuilles d'exercices à distribuer aux élèves, les clés de correction et l'information nécessaire aux enseignants.

Tous ces outils pratiques les rendent fondamentalement prêtes à l'emploi. Pour vous assurer qu'elles répondent aux besoins particuliers de vos élèves, ne manquez pas de lire les remarques à la page suivante. 
ÉTABLISSEMENTS SCOLAIRES : Pour optimiser leur utilité scolaire, la plupart des activités sont conçues en fonction de sessions (une, deux ou trois) de 40 minutes. Comme indiqué à la page précédente, ces activités ont des objectifs scolaires. Leur caractère personnel et agréable peut aussi favoriser la connexité des élèves à l'école.

\section{PROGRAMMES COMMUNAUTAIRES : LeS} activités présentées ici parlent d'« élèves » plutôt que de "participants». Nous avons choisi ce terme pour des raisons de simplicité, mais il ne fait pas strictement référence au contexte scolaire. Nous sommes tous " élèves », à l'école ou non!

\section{FAIBLE NIVEAU D'INSTRUCTION : Beaucoup des} activités impliquent l'écriture, mais la plupart peuvent être adaptées au niveau oral dans les situations où les apprenants sont peu ou non instruits. Beaucoup se prêtent notamment à l'animation de la discussion par présentation d'images (cartes illustrées).

CONTEXTES CULTURELS VARIÉS : Les activités ont été conçues en fonction de nombreux contextes. Vous en trouverez peut-être certaines mieux adaptées que d'autres à vos circonstances. Les instructions suggèrent parfois aussi de changer les noms ou les situations donnés à titre d'exemple pour les rendre plus abordables ou pour mettre les élèves plus à l'aise.
POUR FACILITER LA PLANIFICATION : Chaque activité présente un bref aperçu de l'activité, ses objectifs d'apprentissage, la durée et le matériel requis, ainsi que la préparation éventuellement nécessaire. Des instructions détaillées sont également incluses à lire aux élèves ; celles qui peuvent être adaptées comme vous jugez pratique et confortable.

En lisant deux fois l'activité avant de la présenter, vous vous ferez une idée de la manière dont vous désirez agencer votre leçon. Il vous sera peut-être utile de « faire un essai » sur un groupe de collègues ou d'amis pour vous habituer au format et à l'approche de l'activité. $\mathrm{Ne}$ manquez bien sûr pas de vous familiariser avec le contenu correspondant du GUIDE (ou de votre propre programme).

\section{CONCERNANT LA SÉLECTION D'ACTIVITÉS: CeS} activités ne forment pas un programme en soi. Il s'agit plutôt d'activités types à introduire ou adapter dans votre propre programme ou vos plans de leçon. Sélectionnez les activités qui complètent le mieux votre présentation, documentation écrite, film ou autre composant de votre matière. Les ressources complémentaires proposées en fin de volume incluent des liens à d'autres programmes aussi.

CONCERNANT LE MATÉRIEL : Beaucoup d'activités mentionnent tableau noir et craie comme matériel nécessaire à l'activité. Papier et marqueurs, tableau blanc ou autre surface de taille adéquate peuvent bien sûr s'y substituer.

CONCERNANT LA DURÉE : La durée des activités peut varier suivant la taille et les caractéristiques du groupe et le temps consacré à la discussion. Si les instructions le prévoient, le temps de discussion recommandé en fin d'activité est important. 


\section{pourquoi l'équité compte}

APERCQU : Les élèves réfléchissent à la question de l'équité à travers leur réaction aux citations de quelques grandes personnalités.

OBJECTIFS: Aider les élèves à comprendre l'importance de l'équité et le lien entre l'équité et les droits humains; renforcer l'esprit critique et les aptitudes à l'expression écrite.

\section{DURÉE :}

Étapes $1-2: 15$ minutes (peuvent être données comme devoir la veille de l'étape 3)

Étapes 3-7: 40 minutes

\section{MATÉRIEL :}

Tableau noir + craie

\section{PRÉPARATION :}

Passez en revue les citations proposées. Si vous avez accès à une photocopieuse, faites en une copie pour chaque élève. Écrivez sinon toutes les citations au tableau avant la classe. Décidez éventuellement de donner les étapes 1 et 2 comme devoir avant l'étape 3.

\section{INSTRUCTIONS}

1 Expliquez que ces citations sont celles de personnalités originaires du monde entier. Invitez les élèves à les lire à haute voix, une à la fois, sans les discuter à ce stade.

\section{La justice, c'est l'équité.}

Les femmes ne meurent pas à cause de maladies que l'on ne peut traiter; elles meurent parce que les sociétés n'ont pas encore décidé que leur vie vaut la peine d'être sauvée.

L'avenir aura pour guide notre équité envers les enfants des autres.

Fais preuve de gentillesse envers tous ceux que tu rencontres, leur combat est peut-être plus dur que le tien.

Sois juste envers les autres, mais qu' ils soient justes envers toi aussi.

C'est l'équité qui fait briller les étoiles.

Vis pour que ton nom devienne synonyme d'équité et de respect.

Dans nos coeurs et dans nos lois, nous devons traiter tous les êtres avec équité et dignité, quels que soient leur race, leur genre, leur âge, leurs convictions politiques ou leur religion.

2 Lisez ces instructions à vos élèves :

- Choisissez une citation qui vous inspire.

- Rédigez au moins une page pour expliquer ce que cette citation veut dire pour vous et pourquoi elle vous inspire.

- Incluez un exemple tiré de votre propre vie, ou une situation qui s'est présentée dans votre communauté ou ailleurs, où ce message aurait été utile. Y a-t-il, par exemple, un moment où vous avez été maltraité, où vous n'avez pas fait preuve d'équité envers quelqu'un, ou bien avezvous peut-être observé un échange entre d'autres personnes?

- En quoi le message de la citation choisie aurait-il pu être utile?

- Si vous le désirez, l'occasion vous sera donnée de lire votre composition devant le reste du groupe.

- Y a-t-il des questions sur la tâche à accomplir? 
3 Invitez des volontaires à lire à haute voix leur rédaction sur la citation choisie. (Si vous avez déjà relevé et lu les copies, sélectionnez, peut-être, un ou deux élèves pour lancer l'échange.) Remerciez chaque élève de sa participation.

4 Si les deux ou trois premiers élèves ont tous parlé d'un moment où ils n'ont pas été traités équitablement, demandez si quelqu'un a un exemple où une autre personne a été traitée inéquitablement.

5 Remerciez les volontaires et demandez au groupe :

- Est-ce qu'il est toujours facile d'être équitable?

- Regardez la dernière citation (« Dans nos coeurs...»)

- Dans nos cœurs, traitons-nous vraiment tout le monde avec équité et dignité, sans tenir compte de ces différences? Et nos lois, traitent-elles tout le monde avec équité et dignité ? Pouvez-vous en donner un exemple? [Si possible, encouragez les élèves à penser aux exemples de leur propre société, ainsi qu'à d'autres, basés sur ce qu'ils ont entendu dire d'autres pays.]

6 Dites aux élèves :

- Tout le monde n'est pas toujours d'accord sur ce que c'est que de se traiter les uns les autres avec équité et dignité ou sur la manière dont chacun a le droit d'être traité. Depuis 50 ans pourtant, la communauté mondiale reconnaît que chaque personne a le droit d'être traitée avec respect et dignité.

- Au nom de l'équité, chaque personne mérite une série de protections et droits fondamentaux. Ces droits sont appelés les « droits humains». Ils concernent la manière dont nous nous traitons les uns les autres en tant qu'individus et que membres de la société, simplement parce que nous sommes des êtres humains.

7 Posez les questions suivantes et ouvrez la discussion :

- Pouvez-vous penser à un droit fondamental que chaque personne devrait avoir? [Sondez : nourriture, abri, éducation et sécurité.]

- Est-ce que tout le monde bénéficie effectivement de ces droits fondamentaux?

- Revenez un moment à la citation que vous avez choisie. Cette citation sur l'équité s'applique-t-elle à l'idée plus large des droits humains ?

\section{INFO ENSEIGNANT}

Les citations proposées pour cette activité reviennent (dans l'ordre de leur apparition sous le point 1): au juge américain Potter Stewart ; au médecin égyptien Mahmoud Fathalla ; à la militante des droits de l'enfant et défenseur des droits civiques Marian Wright Edelman ; au philosophe grec Platon; à l'acteur américain Alan Alda ; anonyme; à l'écrivain américain H. Jackson Brown, Jr ; et à l'ancien président des États-Unis Bill Clinton. 


\section{introduction aux droits humains}

\section{DURÉE :}

Étapes 1-2: 40 minutes

Étapes 3-5 : 45 minutes

\section{MATÉRIEL :}

Tableau noir + craie ; au moins un exemplaire des feuilles « Info enseignant » sur la Déclaration universelle des droits de I'homme.

\section{PRÉPARATION :}

Cette activité peut être utile après l'étude des valeurs reflétées dans les droits humains, telles que l'égalité et la non discrimination. Lisez la Déclaration universelle des droits de l'homme et veillez à bien comprendre le sens de chaque droit.
APERC̣U : Les élèves dressent la liste des droits qu'ils voudraient voir dans un nouveau pays imaginaire et la compare à la Déclaration universelle des droits de l'homme. Ils interviewent des adultes sur ce qu'ils entendent par « droits humains ».

OBJECTIFS : Apprendre aux élèves à décrire le concept des droits humains et à en citer plusieurs fondamentaux; renforcer la réflexion abstraite.

\section{INSTRUCTIONS}

1 Répartissez les élèves en groupes de quatre ou cinq personnes et dites-leur :

- Vous allez vivre sur une nouvelle île où tout existe pour permettre la vie humaine. Cette île était jusque là inhabitée. Elle n’a aucune loi ni aucune histoire.

- Vous devez dresser une liste de 10 droits humains qui s'appliqueront automatiquement à tous les habitants de l'île.

- Aucun d'entre vous ne sait quels seront votre titre, votre genre, votre ethnicité ou race, votre classe sociale ou votre orientation sexuelle. Vous avez 10 minutes pour dresser votre liste.

2 Invitez chaque groupe à présenter sa liste. Compilez une liste globale incluant tous les droits mentionnés.

3 Présentez la Déclaration universelle des droits de l'homme. Expliquez qu'il s'agit d'une liste des droits applicables à tous les habitants du monde. Invitez chaque élève à lire un " article » à haute voix. En fin de liste, demandez s'il y a des questions.
4 Redivisez les élèves en petits groupes et donnez-leur 10 minutes de réflexion sur ces questions :

- Quelles sont les similarités entre notre liste et celle de la Déclaration des droits de l'homme?

- À quels droits de la Déclaration universelle n’avons-nous pas pensé?

- Aimerions-nous ajouter des droits à notre liste?

- Y a-t-il sur notre liste des droits non repris dans la Déclaration universelle?

5 Rassemblez la classe et passez en revue les réponses des groupes aux questions de l'étape 4 . Demandez l'importance d'une liste de droits humains universellement acceptée et applicable.

Devoir : Interviewez deux adultes sur la question : "Que veut dire pour vous l'expression droits humains? Pouvez-vous me donner un exemple de problème de droits humains dont vous avez entendu parler? » Par écrit, résumez la question soulevée par la personne, ce que vous avez appris et quelle intervention vous paraîtrait utile. 


\section{la Déclaration universelle des droits de l'homme en langage courant}

Cette liste explique chaque droit humain en langage courant.

Texte officiel en français sur <www.ohchr.org/EN/UDHR/Pages/Language.aspx?LangID=frn>.

ARTICLE 1: Quand les enfants naissent, ils sont libres et tous doivent être traités de la même manière. Ils sont doués de raison et de conscience, et doivent agir les uns envers les autres de façon amicale.

ARTICLE 2: Les droits de la Déclaration sont reconnus à tout le monde, sans différence de :

- genre ;

- couleur de peau ;

- langue ;

- convictions politiques;

- religion ;

- richesse ou pauvreté ;

- groupe social;

- pays de naissance et

- état indépendant ou non de son pays.

ARTICLE 3: Vous avez le droit de vivre, et de vivre libre et en sécurité.

ARTICLE 4: Personne n'a le droit de vous prendre comme esclave et vous ne pouvez prendre personne comme esclave.

ARTICLE 5: Personne n'a le droit de vous torturer.

ARTICLE 6: Vous devez être protégé par la loi de la même manière, partout et comme tout le monde.

ARTICLE 7 : La loi est la même pour tous ; elle doit être appliquée de la même manière pour tous.

ARTICLE 8: Vous devez pouvoir demander la protection de la justice quand les droits que votre pays vous reconnaît ne sont pas respectés.

ARTICLE 9: Personne n'a le droit de vous mettre en prison, de vous y garder ou de vous renvoyer de votre pays injustement ou sans raison.

ARTICLE 10 : Si vous devez être jugé, ce doit être publiquement. Ceux qui vous jugent doivent être libres de toute influence. 
la Déclaration universelle des droits de l'homme en langage courant

ARTICLE 11: Vous devez être considéré innocent jusqu'à preuve du contraire. Si vous êtes accusé d'un crime, vous devez toujours avoir le droit de vous défendre. Personne n’a le droit de vous condamner ou de vous punir pour quelque chose que vous n'avez pas fait.

ARTICLE 12: Vous avez le droit de demander à être protégé si quelqu'un essaie de porter atteinte à votre réputation, d'entrer dans votre maison, d'ouvrir votre courrier ou de vous importuner, vous ou votre famille, sans raison.

ARTICLE 13: Vous avez le droit de circuler comme bon vous semble dans votre pays. Vous avez le droit de quitter votre pays pour vous rendre dans une autre, ainsi que d'y revenir si vous le désirez.

ARTICLE 14 : Si quelqu'un vous fait du mal, vous avez le droit de chercher asile dans un autre pays. Vous pouvez perdre ce droit si vous commettez une infraction grave aux droits humains.

ARTICLE 15 : Vous avez le droit d'appartenir à un pays et personne ne peut vous empêcher sans raison de changer de nationalité si vous le désirez.

ARTICLE 16: Dès que la loi vous le permet, vous avez le droit de vous marier et de fonder une famille. Ni la couleur de votre peau, ni votre pays d'origine, ni votre religion ne peuvent y faire obstacle. Les hommes et les femmes ont les mêmes droits quand ils sont mariés et aussi quand ils se séparent. On ne peut forcer personne à se marier. Le gouvernement de votre pays doit protéger votre famille et ses membres.

ARTICLE 17 : Vous avez le droit de posséder quelque chose et personne n’a le droit de vous le prendre sans raison.

ARTICLE 18: Vous avez le droit de choisir votre religion, d'en changer et de la pratiquer seul ou avec d'autres personnes.

ARTICLE 19: Vous avez le droit de penser et de dire ce que vous voulez, et personne ne peut vous l'interdire. Vous avez le droit d'échanger librement vos idées, y compris avec les habitants d'autres pays.

ARTICLE 20 : Vous avez le droit d'organiser des réunions pacifiques ou de participer à de telles rencontres. Personne n'a le droit de vous forcer à devenir membre d'un groupe.

ARTICLE 21: Vous avez le même droit que toute autre personne de participer à la vie politique de votre pays, en devenant vous-même membre du gouvernement ou en choisissant des représentants qui ont les mêmes idées que vous. Les gouvernements doivent être élus régulièrement et le vote doit être secret. Vous devez être autorisé à voter et toutes les voix doivent avoir la même valeur. 


\section{la Déclaration universelle des droits de l'homme en langage courant}

ARTICLE 22: La société dans laquelle vous vivez doit vous aider à profiter de tous les avantages (culture, emploi, protection sociale) offerts à tous et à toutes dans votre pays et à les développer.

ARTICLE 23 : Vous avez le droit de travailler, de choisir librement votre travail et de toucher un salaire qui vous permette de vivre et de subvenir aux besoins de votre famille. Un homme et une femme qui accomplissent un même travail doivent toucher un salaire égal. Tous ceux qui travaillent ont le droit de s'unir dans le but de protéger et de défendre leurs intérêts.

ARTICLE 24 : La durée de chaque jour de travail ne doit pas être excessive, car chacun a droit au repos et à des congés payés périodiques.

ARTICLE 25: Vous avez le droit, vous et votre famille, d'avoir ce qu'il faut pour ne pas tomber malade, manger à votre faim, vous habiller et vous loger et vous avez le droit d'être aidé(e) si vous êtes au chômage, malade, âgé(e), veuf(ve) ou que vous ne pouvez pas gagner votre vie pour d'autres raisons indépendantes de votre volonté. Une mère qui va avoir un enfant et son enfant doivent bénéficier d'une assistance particulière. Tous les enfants ont les mêmes droits, que leur mère soit mariée ou non.

ARTICLE 26: Vous avez le droit d'aller à l'école et tous devraient pouvoir y aller. L'école primaire doit être gratuite. Vous devez pouvoir apprendre un métier ou une profession ou faire les études que vous désirez. À l'école, vous devez pouvoir développer toutes vos aptitudes et apprendre à vous entendre avec les autres, quelles que soient leur race, leur religion ou leurs origines. Vos parents ont le droit de choisir ce qui vous est enseigné et comment.

ARTICLE 27: Vous avez le droit de participer à la vie artistique et scientifique de votre communauté, ainsi qu'à ses bienfaits. Si vous êtes artiste, auteur ou scientifique, vos travaux doivent être protégés et vous devez pouvoir en tirer profit.

ARTICLE 28: Pour assurer le respect de vos droits, un « ordre» doit être établi dans le but de les protéger. Cet « ordre » doit régner au niveau local aussi bien que mondial.

ARTICLE 29: Vous avez des devoirs envers votre communauté. La loi doit garantir vos droits humains. Elle doit permettre à chacun de respecter les autres et d'être respecté.

ARTICLE 30 : Aucune société et aucun être humain, nulle part au monde, ne peut se permettre de détruire les droits énumérés ici.

Cette activité est adaptée de ABC : L'enseignement des droits de l'homme - Activités pratiques pour les écoles primaires et secondaires (Bureau du Haut Commissariat aux Droits de I'Homme. 2004. New York et Genève ONU). Cette liste (adaptée en partie de celle de l'Association mondiale pour l'école instrument de paix de I'Université de Genève) explique chaque droit humain en langage courant. Le texte officiel en français peut être consulté sur < $w$ ww. ohchr. org/EN/UDHR/Pages/Language. aspx? Lang $\mid D=f r n>$. 


\section{pouvoir, privilège et égalité}

\section{DURÉE :}

45 minutes

\section{MATÉRIEL :}

Tableau noir + craie

\section{PRÉPARATION :}

Ajoutez à la liste de droite tous les groupes pertinents de votre communauté. Achevez l'étape 1 avant la réunion de la classe.
APERCQU : Les élèves réfléchissent au rapport entre le pouvoir social et le privilège ou la discrimination.

OBJECTIFS : Aider les élèves à parler de l'agencement du pouvoir dans la société, à identifier la manière dont le pouvoir affecte le privilège, la discrimination et l'oppression, et à rapporter ces questions à leur propre vie ; renforcer leur esprit d'analyse et leur aptitude à la résolution de problèmes.

\section{INSTRUCTIONS}

1 Tracez un tableau en deux colonnes au tableau noir. Intitulez la première colonne «Plus de pouvoir/ privilège » et la seconde, «Moins de pouvoir/ privilège ». Expliquez aux élèves qu'ils vont discuter les concepts du pouvoir, du privilège et de l'égalité dans leur vie et dans la société.

2 Dans la liste « Groupes ayant généralement un pouvoir inégal dans la société », lisez le premier exemple (les riches et les pauvres).

3 Demandez: Quel groupe faut-il inscrire sous «Plus de pouvoir »? Et sous « Moins de pouvoir »? Rappelez aux élèves que ces caractérisations ne sont pas toujours vraies ni absolues.

4 Inscrivez la réponse des élèves dans la colonne appropriée et ainsi de suite pour chaque élément de la liste. (Pour les groupes ethniques, raciaux et religieux, mentionnez-les par leur appellation spécifique dans votre région.)
Groupes ayant généralement un pouvoir inégal dans la société :

Riches / Pauvres

Hommes / Femmes

Hétérosexuels / Homosexuels

Patrons / Ouvriers

Hommes politiques / Membres de la communauté

Citoyens / Réfugiés

Non-handicapés / Handicapés

Majorité ethnique / Minorité ethnique

Majorité religieuse / Minorité religieuse

Personnes jugées belles / Personnes jugées laides 


\section{Demandez:}

- Considérez la liste des groupes généralement plus privilégiés. Y voyez-vous un groupe auquel vous vous identifiez personnellement ? Pensez-vous que ce groupe a généralement plus de pouvoir dans la société ? En quelques phrases, décrivez une situation où vous avez observé ou entendu parler de cette différence de pouvoir. [Prévoyez 3 à 5 minutes de temps de rédaction.]

- Considérez la liste des groupes généralement moins privilégiés. Y en a-t-il un avec lequel vous vous identifiez personnellement? Pensez-vous qu'il est en effet moins privilégié ? Rédigez un paragraphe décrivant une situation où vous avez vécu ou entendu parler de cette différence. [Prévoyez 3 à 5 minutes de temps de rédaction.]

- Levez la main si vous vous identifiez avec au moins un groupe de chaque liste. Savons-nous, pour la plupart, ce que c'est que d'être À LA FOIS plus privilégiés et moins privilégiés?

6 Invitez quelques volontaires à lire leur récit d'appartenance à un groupe moins privilégié. ( $\mathrm{Ne}$ forcez personne à participer.)

7 Discussion (prenez 1-2 réponses par question) :

- Que remarquez-vous sur le traitement reçu par les personnes des groupes moins privilégiés? [Sondez : ceux qui ont moins de pouvoir sont souvent victimes de discrimination ou d'oppression.]

- Quelles émotions ce traitement peut-il susciter chez ceux moins puissants? [Listez les émotions citées au tableau. Veillez à écrire des noms

d'émotion, pas leur description.]

- Que remarquez-vous dans les sentiments ou les émotions listés?

8 Revenez aux deux listes et demandez:

- Pensez au concept de l'égalité. Prenez la première paire (riches / pauvres), par exemple. Comment pourrait-on achever la phrase : «L'égalité entre les riches et les pauvres___ " ? [Plusieurs réponses peuvent être correctes.]

- Qui pourrait achever la même phrase pour une autre paire? Essayez des mots différents, ou développez une autre idée. [Répétez pour plusieurs paires, suivant le temps disponible.]

- Qui se souvient du premier article de la Déclaration universelle des droits de l'homme? [ = le droit à l'égalité. Écrivez cet article au tableau: "Tous les êtres humains naissent libres et égaux en dignité et en droits. Ils sont doués de raison et de conscience et doivent agir les uns envers les autres dans un esprit de fraternité. »]

9 Clôturez la discussion sur la question suivante (ou encouragez simplement les élèves à y réfléchir au moment de quitter la classe) : Que faut-il pour assurer l'égalité et le droit de tous à la dignité?

Devoir : Répondez par écrit à la question : Que pourriez-vous faire dans votre vie de tous les jours pour promouvoir l'égalité et reconnaître la dignité de chaque être humain?
Adaptation de Helping Teens Stop Violence: A Practical Guide for Counselors, Educators, and Parents (Allan Creighton et Paul Kivel. 1990, 1992. Alameda, CA: Hunter House Inc.). Pour commander (en anglais): $<$ www.hunterhouse.com/shopexd. asp?id $=308 \& b c=$ no $>$. 


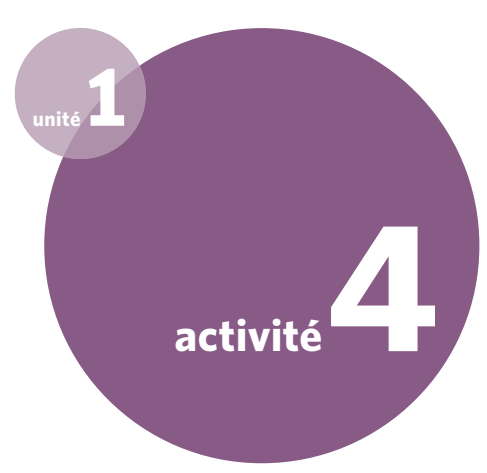

\section{DURÉE :}

40-60 minutes, suivant que deux ou trois études de cas sont analysées.

\section{MATÉRIEL :}

Tableau noir + craie ; un exemplaire de chaque cas à analyser (avec, éventuellement,

la première partie au recto

et la seconde au verso).

\section{PRÉPARATION :}

Cette activité est utile après la présentation aux élèves de la notion de droits sexuels et génésiques. Sélectionnez trois études de cas à la page suivante ou choisissez des cas propres à votre pays. Écrivez à l'avance les questions de l'étape 2 au tableau noir.

\section{études de cas sur les droits sexuels et génésiques}

APERC̣U : Les élèves analysent et discutent des histoires vécues de violation des droits sexuels ou génésiques.

OBJECTIFS : Apprendre aux élèves à décrire combien la capacité d'exercer ses droits humains affectent les relations intimes et la sexualité ; renforcer la réflexion critique.

\section{INSTRUCTIONS}

1 Nous allons discuter aujourd'hui quelques histoires vécues sur la question des droits sexuels et génésiques. Invitez un volontaire à lire à voix haute la première partie (seulement) de l'histoire d'Alicja.

2 Cela fait, invitez les élèves à répondre par écrit aux questions suivantes. Lisez à haute voix les questions que vous avez écrites au tableau noir :

- Quelle est votre réaction à ce récit ?

- Quels sont les droits sexuels ou génésiques impliqués?

- S'agit-il d'une violation des droits humains? Pourquoi?

- Qui est responsable ? Dressez la liste de tous les responsables de la situation.

3 Pour chaque question, invitez un ou deux volontaires à lire leur réponse puis invitez les commentaires.

4 Demandez au lecteur du récit de lire la deuxième partie («Qu'est-il arrivé ? ) et lancez la discussion :

- Que pensez-vous du résultat?
- Comment les choses auraient-elles pu être différentes si la victime avait été riche (ou de sexe masculin, ou hétérosexuelle) ?

- Quelles sont les attitudes qui doivent changer si l'on veut protéger ce droit sexuel?

5 Répétez ces étapes pour chaque étude de cas sélectionnée. (Prévoyez 10 minutes par cas.)

6 Après le dernier cas, posez les questions suivantes pour conclure la leçon :

- Êtes-vous au courant de cas similaires qui se sont produits ici ? Quels droits ont été violés?

- Avez-vous entendu parler d'autres types de violations des droits sexuels dans notre pays ou ailleurs?

- Comment mettre fin à ces violations ?

- Quelle est la conclusion à tirer au sujet du rapport entre les droits humains et notre vie intime, amoureuse et sexuelle? [Écrivez la conclusion finale au tableau noir.] 


\section{études de cas sur les droits sexuels et génésiques}

\section{ALICJA}

Première partie : L'histoire d'Alicja : Quand Alicja, une Polonaise souffrant de problèmes de vision depuis l'enfance, se retrouve enceinte, plusieurs médecins lui disent que sa grossesse et l'accouchement risquent de lui endommager irréversiblement la vue. En Pologne, la loi autorise l'avortement quand la santé de la femme est en danger. Les médecins refusent cependant de donner à Alicja le certificat nécessaire et elle n’a donc d'autre choix que de mener sa grossesse à terme.

\section{Deuxième partie : Qu'est-il arrivé à Alicja ? La} naissance a provoqué une plus grande détérioration de sa vue. Il lui est devenu impossible de travailler et de s'acquitter, seule, de ses tâches journalières et des soins de ses enfants. Elle est devenue totalement dépendante de l'assistance publique. La cause d'Alicja a été entendue devant la Cour européenne des Droits de l'homme, qui a statué que les États sont tenus d'établir des mécanismes d'accès à l'avortement là où il est légal. Reconnaissant la souffrance imposée à Alicja, la cour lui a accordé d'importants dommages-intérêts.

\section{AMINA}

Première partie : L'histoire d'Amina : Amina, Nigériane divorcée mère de trois enfants, rencontre Mohammed. Au bout de 11 mois, il lui demande des rapports sexuels et lui promet de l'épouser. Elle accepte et se retrouve enceinte. Mais Mohammed ne tient pas parole et elle devient mère célibataire, accusée d'adultère par la loi religieuse. Mohammed jure qu'il n'est pas le père et est acquitté. Amina est, elle, reconnue coupable et condamnée à mort par lapidation. Elle fait appel mais le verdict est confirmé. Son exécution est différée pendant deux ans pour lui permettre d'allaiter sa fille.

Deuxième partie : Qu'est-il arrivé à Amina ? En second appel, Amina est acquittée et sa sentence est révoquée. Les juges reconnaissent qu'il ne lui a pas été donné de défendre suffisamment sa cause. Le gouvernement nie la sentence de lapidation. Amina s'est depuis lors remariée. 


\section{études de cas sur les droits sexuels et génésiques}

\section{FATIMA}

Première partie : L'histoire de Fatima : Fatima, fillette ouest-africaine de 11 ans, entend ses parents parler de son excision. Elle prend peur car elle a vu sa sœur aînée, souffrante et malheureuse, au retour de sa cérémonie. Elle pense aussi à sa meilleure amie, en proie à de graves infections depuis son excision. Elle ne veut pas vivre l'expérience endurée par tant de ses semblables et elle supplie ses parents de ne pas l'y forcer. Ceux-ci hésitent à l'écouter car ils craignent qu'elle ne soit pas mariable si elle n'est pas excisée. Ils ne pensent pas non plus que le choix doive être laissé à un être aussi jeune et inexpérimenté. Or la sœur de Fatima a entendu parler d'une organisation locale qui informe les familles sur les dangers et les risques pour la santé de la mutilation génitale des filles (MGF). Elle invite une représentante de l'organisation à rendre visite à ses parents et à leur parler de la situation de Fatima.

Deuxième partie : Qu'est-il arrivé à Fatima ? La représentante de l'organisation a su convaincre les parents de Fatima des dangers posés par l'excision, leur présentant d'autres façons de célébrer le rite de passage de leur fille à l'état de femme. Fatima est aujourd'hui mariée et heureuse, reconnaissante à ses parents d'avoir fait preuve d'une telle ouverture d'esprit. Elle travaille elle-même pour l'organisation qui l'a aidée à éviter la MGF, éduquant les écolières sur la manière de parler de l'excision à leurs parents.

\section{MATTHEW}

Première partie : L'histoire de Matthew. Matthew était un étudiant homosexuel, dans une université américaine. Un soir, deux jeunes hommes ont fait semblant d'être homosexuels aussi et lui ont proposé de le ramener chez lui après avoir pris un verre dans un bar. Mais ils l'ont emmené dans un endroit isolé et, l'ayant attaché à une barrière, l'ont battu violemment et l'ont torturé, le laissant pour mort. Matthew a été retrouvé 18 heures plus tard, toujours lié à la barrière, par un cycliste qui avait d'abord cru voir un épouvantail. Matthew vivait encore, mais il était dans le coma.

Deuxième partie : Qu'est-il arrivé à Matthew? Le crâne fracturé et le cerveau gravement atteint, Matthew n'a jamais repris conscience et est mort cinq jours plus tard. Ses agresseurs ont été arrêtés, et finalement condamnés, chacun, à deux peines consécutives d'incarcération à vie. L'histoire de Matthew a attiré l'attention nationale des États-Unis sur le problème des crimes haineux. Une loi adoptée sous le nom de Matthew a étendu la législation relative aux crimes haineux à ceux perpétrés à l'encontre des homosexuels, des femmes et des personnes handicapées. La mère de Matthew a créé en son nom la Fondation Matthew Shepard, qui cherche à « remplacer la haine par la compréhension, la compassion et l'acceptation » à travers l'éducation, la sensibilisation et le plaidoyer. 


\section{études de cas sur les droits sexuels et génésiques}

\section{LAKSHMI}

Première partie : L'histoire de Lakshmi. Lakshmi, jeune Népalaise forcée à se marier à l'âge de 12 ans, est ainsi tombée sous le joug de la famille de son mari. Incapable de supporter cette exploitation, elle s'est enfuie et a cherché refuge chez ses parents, qui l'ont aussitôt renvoyée à son mari. «J'ai réussi à m'échapper en chemin, » dit-elle, « avec l'aide d'une gentille dame dont la sœur travaillait dans une usine de vêtements et que j'aurais pu aider». Mais Lakshmi allait en fait être droguée et emmenée en Inde. «J'ai appris que j’avais été vendue pour 15 mille roupies ", se souvientelle. «On m’a battue quand j'ai refusé de devenir une travailleuse du sexe. Pendant un an, je suis restée prisonnière dans une maison close. Un beau jour, la police a fait une descente ; j'ai été libérée et renvoyée au Népal. J’avais 14 ans.»

Deuxième partie : Qu'est-il arrivé à Lakshmi ? À son retour, les parents de Lakshmi ont refusé de l'accueillir. Elle s'est remariée mais un test de dépistage l'a révélée séropositive à VIH. Il n'a pas été établi si elle avait contracté le virus pendant son année de prostitution ou après son mariage. 


\section{toiles de mots homme-femme}

\section{DURÉE :}

45 minutes

\section{MATÉRIEL :}

Tableau noir + craie

\section{PRÉPARATION :}

Décidez s'il convient d'ajouter d'autres caractéristiques propres à votre communauté.
APERCQU : En petits groupes, les élèves créent des toiles de mots que la société associe à la « masculinité » ou à la « féminité » et ils discutent le fondement de leurs idées. Ils découvrent la notion de genre.

OBJECTIFS: Aider les élèves à définir le « genre » et à distinguer les caractéristiques masculines et féminines biologiques de celles dictées par les normes sociales; renforcer la réflexion critique et les aptitudes à l'expression écrite.

\section{INSTRUCTIONS}

1 Divisez la classe en groupes de quatre ou cinq élèves (de même sexe ou non).

2 Expliquez:

- Nous allons parler aujourd'hui du « genre» (définition sociale de l'homme et de la femme). Chaque groupe va créer des toiles de mots souvent associés au fait d'être un homme ou une femme.

Pour clarifier le concept de " toile de mots», donnez-en un exemple au tableau, sur un autre sujet. Voir l'exemple illustréci-dessous pour le mot «chien».

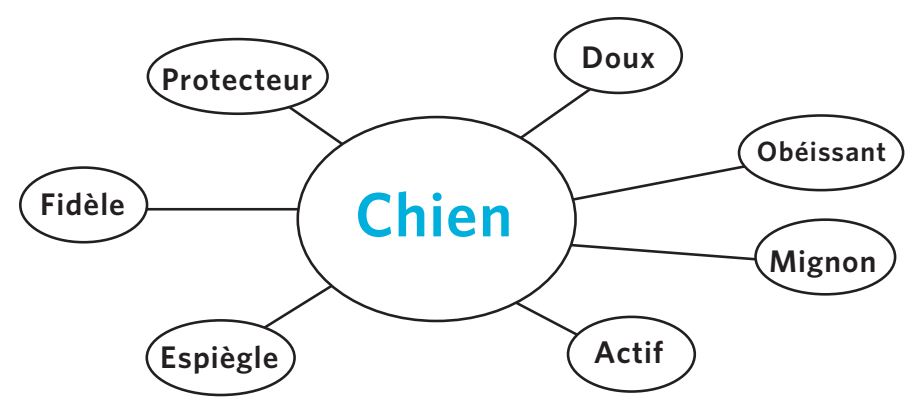

3 Donnez à chaque groupe deux à trois minutes pour la création d'une toile « homme», puis deux à trois minutes pour celle d'une toile «femme ».

4 Écrivez «Femme » et « Homme » au tableau et tracez sous chaque mot deux colonnes intitulées «biologique » et « social ». Commencez par un

- Donnez-moi une caractéristique que vous avez associée sur votre toile au mot « homme».

- S'agit-il d’une caractéristique « biologique » ou «sociale»?

- Si les élèves placent une caractéristique « sociale " dans la catégorie « biologique », corrigez-les en demandant: si un garçon ou un homme ne possède pas cette caractéristique, est-il toujours de sexe masculin?

5 Ajoutez une nouvelle caractéristique proposée par chaque groupe jusqu'à obtenir toutes celles qui définissent l'homme. Assurez-vous de la présence de beaucoup des mots suivants. (Au groupe et demandez : 
besoin, sondez pour obtenir les réponses voulues.)

Exemples courants de caractéristiques associées à la masculinité :

- Force physique

- Absence d'expression affective

- Prédateur sexuel

- Hétérosexuel

- Réussite financière

- Chef de famille

- Décontracté

- Père

- Fier

- Puissant

- Athlétique

- Courageux

- Violent

- Plein d'humour

- Loyauté envers ses amis

6 Répétez l'approche pour les caractéristiques associées à la féminité. Exemples courants :

- Attentionnée

- Silencieuse

- Soumise

- Bavarde

- Bonne communicatrice

- Apparence soignée

- Psychologiquement forte

- Bien organisée/capable de faire plusieurs choses à la fois

- Pratique

- Non violente

- Modeste
- Bien balancée

- Physiquement faible par rapport à l'homme

- Aimante

- Mère

7 Réservez 10 minutes à la discussion au complet :

- Quelques caractéristiques masculines et féminines sont de nature biologique : le fait d'être père ou de pouvoir allaiter, par exemple.

- La plupart sont cependant déterminées par la société, sans fondement biologique.

- Les rôles masculins et féminins d'origine sociale sont des « rôles de genre ». Qui a déjà entendu cette expression?

- Que pensez-vous des rôles de genre dans notre société? Êtes-vous d'accord avec tous les aspects de la façon dont les femmes sont censées agir et vivre? Et les hommes ?

- Que veut dire, selon vous, l'égalité de genre?

- Dans chaque communauté et chaque société, certaines personnes ont des attitudes de genre et d'égalité non conventionnelles.

- L'évolution sociale, dans le temps et dans l'espace, s'accompagne de celle des attitudes à l'égard des rôles de genre.

Devoir : Achever et développer l'énoncé suivant, sous forme de liste, de lettre ou de poème : «Être fille (ou garçon), ce n'est pas facile parce que...»
Adaptation de What's the Real Deal About Masculinity? (2008. Scenarios USA.) Pour commander (en anglais) $<$ www.scenariosusa.org/getinvolved/ MasculinityCurriculum2009.html> 


\section{souvenirs d'enfance : I'apprentissage du genre}

activité

\section{DURÉE :}

45 minutes (l'étape 5 peut être donnée comme devoir)

\section{MATÉRIEL :}

Tableau noir + craie

\section{PRÉPARATION :}

Plongez-vous vous-même dans vos souvenirs d'enfance avant de mener cette activité. Les appels au souvenir peuvent réveiller des sentiments pénibles pour certains élèves. Réfléchissez à la meilleure façon d'y répondre - et à qui vous adresser si un élève a besoin d'aide.
APERCU : Les élèves partagent un souvenir d'un moment où ils ont été traités d'une certaine manière en raison de leur sexe et évoquent leurs sentiments.

OBJECTIFS : Aider les élèves à produire des exemples de la manière dont les enfants absorbent les messages relatifs aux rôles de genre ; considérer ces messages d'un point de vue personnel et dans l'optique des droits humains; renforcer la réflexion critique.

\section{INSTRUCTIONS}

1 Divisez la classe en groupes de quatre à cinq élèves de même sexe. Dites-leur de prendre un stylo et du papier.

2 Expliquez:

- Nous allons explorer aujourd'hui ce que c'est que de grandir garçon ou fille. Commençons par un bref retour dans le temps. Oubliez un instant vos stylos et installez-vous confortablement.

- Y a-t-il, dans vos souvenirs, un moment où vous avez été traités ou traitées différemment en raison de votre sexe ? Écrivez ce qui vous revient.

- Quand vous vous rappelez de cette expérience, quels sentiments fait-elle naître en vous? Décrivez ces émotions. L'occasion vous sera donnée de partager votre souvenir.

3 Après quelques minutes, dites:

- Dans votre groupe, passez quelques minutes à partager vos expériences ou vos sentiments, mais seulement si vous le désirez.
4 Au bout de cinq ou 10 minutes, demandez :

- Que nous disent ces expériences sur les attitudes et les normes sociales qui dictent la valeur et le rôle des filles et des femmes? Et des garçons et des hommes?

- Dans l'optique des droits humains, ces attitudes et ces normes vous semblent-elles justes ou équitables ? Pourquoi ou pourquoi pas?

- Quels changements faudrait-il adopter pour atteindre l'égalité entre les deux sexes?

5 [Remarque: Si vous manquez de temps, donnez cette étape comme devoir.] Rappelez aux élèves qu'une situation peut avoir différents résultats. Donnez leur l'occasion de changer la fin d'une histoire :

- Revenez au souvenir d'une situation qui vous a paru injuste - celui que vous avez vous-même inscrit sur votre papier ou un autre partagé avec le groupe.

- Rédigez une nouvelle fin à l'histoire, qui vous paraisse plus juste. 


\section{images de père}

APERCU : Les élèves rédigent une lettre à leur père ou tuteur. Ils discutent la manière dont les rôles de genre limitent la paternité et ils décrivent le type de père qu'ils espèrent devenir ou, pour les filles, avoir pour leurs enfants (ou pour d'autres).

OBJECTIFS : Aider les élèves à évaluer leur expérience et leurs valeurs concernant la paternité ; renforcer la réflexion personnelle et les aptitudes à l'expression écrite.

\section{INSTRUCTIONS}

1 Présentez le sujet :

- Nous allons parler aujourd'hui de la paternité et de modèles de rôles masculins.

- J'aimerais que vous commenciez tous par écrire une courte lettre à votre père, tuteur ou autre figure paternelle importante. Vous ne devrez pas partager cette lettre. Commencez votre lettre de l'une des façons suivantes:

Cher Papa, Tu crois que j'ai oublié, mais je me souviens... ou bien

Cher Papa, Je voudrais que tu...

2 Au bout d'environ cinq minutes, demandez:

- Quel genre de personne est ou était votre père, tuteur ou autre homme important dans votre vie ? Écrivez au moins 10 adjectifs qui le décrivent. Ceci restera confidentiel aussi. Vous ne devrez pas le partager.

- Listez maintenant 10 adjectifs que vous aimeriez voir inclus dans le rôle du père, qui décrivent pour vous un excellent père.
- Considérez votre seconde liste. Cochez les adjectifs qui décrivent la plupart des pères. Entourez ceux plus rares, qui décrivent peu de pères.

- Les hommes trouvent-ils que les rôles masculins traditionnels leur facilitent ou non la tâche quand il s'agit d'être le père qu'attendent leurs enfants?

- Connaissez-vous un exemple de père ou figure paternelle qui agit au défi des rôles de genre traditionnels?

3 Pour conclure, demandez: Comment aimeriezvous voir changer la paternité dans le futur?

Devoir : Interviewez un homme adulte que vous connaissez. Demandez-lui de vous décrire ce qu'il pense être de bonnes qualités de père.

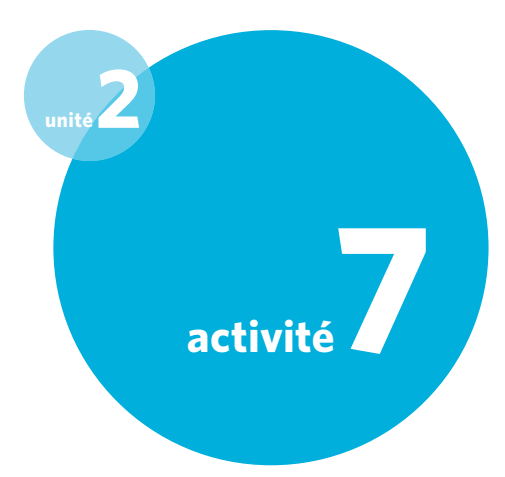

DURÉE :

45 minutes

\section{MATÉRIEL :}

Tableau noir + craie

\section{PRÉPARATION :}

Écrivez les questions et énoncés de l'étape 1 au tableau ou faites en une feuille d'exercice. Pensez à l'impact émotionnel que cette activité peut avoir sur certains élèves (surtout ceux qui éprouvent des sentiments ambivalents ou négatifs à l'égard de leur père ou dont le père est absent). Ne procédez pas sauf si vous pouvez offrir du soutien adéquat aux élèves quand ils en ont besoin. 


\section{projet de recherche : le genre à l'école}

APERCQU : Les élèves mènent une recherche sur le rôle du genre à l'école et présentent leurs conclusions. (Temps de classe + hors classe requis.)

OBJECTIFS: Aider les élèves à discuter la manière dont le milieu scolaire peut renforcer ou défier les normes de genre; renforcer les aptitudes de recherche.

\section{DURÉE :}

Étapes 1-5: 40 minutes

Étape $6: 4$ à 5 heures

Étapes $7-8: 90$ à 120 minutes

\section{MATÉRIEL :}

Tableau noir + craie ; copies des feuilles à distribuer aux élèves ; ruban adhésif

\section{PRÉPARATION :}

Obtenez la permission de la direction de l'école pour la recherche. Inscrivez chaque approche (étape 2) sur une feuille de papier différente. (Au besoin, changez la liste de sujets en fonction de votre contexte scolaire.) Déterminez la manière dont vous allez répartir les élèves par sujet à l'étape 3. Si possible, faites une copie de la feuille à distribuer pour chaque élève.

\section{INSTRUCTIONS}

1 Affichez les feuilles de recherche (par approche) en différents endroits de la salle.

2 Expliquez:

- Vous allez, cette semaine, effectuer une recherche sur la question suivante : «Comment les écoles renforcent ou défient-elles les rôles de genre traditionnels?»

- Chacune de quatre équipes évaluera une approche différente de la question : comportement en classe, activités parascolaires, programmes et manuels et statistiques scolaires.

- Identifiez deux approches qui vous intéressent.

3 Pour former les équipes, invitez les élèves à s'inscrire sur la feuille de l'approche qui les intéresse. (Ou bien désignez vous-même les équipes.)

4 Distribuez et expliquez les instructions. Veillez à ce que tout le monde les comprennent bien.

5 Distribuez les feuilles d'équipe. Invitez les groupes à discuter la manière dont ils entendent mener leur recherche. Circulez parmi les groupes pour les aider.
6 Accordez jusqu'à deux semaines à la phase de recherche. Continuez à offrir l'aide demandée.

7 La phase de recherche terminée, invitez les élèves à présenter leurs résultats, sous forme d'exposés de 15 minutes chacun. Après chaque exposé, demandez aux autres élèves :

- Avez-vous des questions à poser à cette équipe ?

- Qu'est-ce qui vous parait particulièrement intéressant ou surprenant?

- Êtes-vous d'accord avec leurs conclusions? Si non, pourquoi?

- Quelles seraient vos recommandations?

8 Après tous les exposés, demandez à la classe :

- Comment le milieu scolaire renforce-t-il les normes de genre traditionnelles?

- Et comment les défie-t-il ?

- Citez cinq changements qui rendraient l'école plus juste en termes de genre, plus accueillante, respectueuse et sûre pour tous.

- Comment pouvez-vous concrétiser ce changement? 


\section{instructions pour toutes équipes}

L'objet général du projet est : « Le rôle du genre à l'école ». Chaque équipe va mener sa recherche sur un aspect différent de ce thème, selon la procédure suivante :

1 Lisez attentivement les questions avec votre équipe.

2 Discutez la meilleure façon d'y trouver la réponse. N'hésitez pas à demander l'aide de votre instructeur.

3 Décidez si chaque membre de l'équipe doit assumer la responsabilité d'une question différente, ou si tous mèneront en coopération la recherche relative à certaines questions.

4 Collectez l'information nécessaire pour répondre aux questions. N'hésitez pas, s'il le faut, à demander de l'aide.

5 Après avoir recueilli vos données, rédigez votre rapport en y incluant les sections suivantes :

a La ou les questions auxquelles vous avez essayé de répondre

b Vos méthodes de recherche (comment vous avez mené votre recherche)

c Vos résultats (ce que vous avez appris)

d Votre conclusion (ce que vous pensez de vos résultats, quelles conclusions vous en tirez)

e Vos recommandations (les changements qui rendraient le milieu scolaire plus juste en termes de genre et plus sûr et accueillant pour les filles comme pour les garçons)

Votre équipe doit présenter un exposé de 15 minutes à la classe sur la base de son rapport. Préparez votre exposé. Veillez à ce que tous les membres de l'équipe y participent. 


\section{ÉQUIPE 1}

Pensez-vous que nos manuels sont sexistes ou qu'ils favorisent l'égalité de genre?

\section{Questions types:}

- Comment les filles, les garçons, les hommes et les femmes sont-ils représentés dans nos manuels?

- Combien de filles/femmes figurent sur les images et combien de garçons/hommes?

- Les accomplissements historiques, intellectuels, scientifiques, littéraires et artistiques des femmes sont-ils pleinement représentés?

- Dans les récits et la littérature, qui représente l'action? Et la subordination?

- Quel est le point de vue du manuel ? Son auteur estil un homme ou une femme?

- De quelles autres manières les stéréotypes de genre sont-ils reflétés et renforcés ou défiés?

- Y a-t-il d'autres groupes stéréotypés dans le manuel?

\section{Méthodes de recherche:}

Prenez au moins deux manuels scolaires (ceux d'études sociales, d'histoire ou de littérature de l'enseignement primaire ou secondaire, par exemple). Analysez le contenu et les images pour répondre aux questions ci-dessus.

\section{ÉQUIPE 2}

Comment la classe renforce ou défie-telle les rôles de genre conventionnels?

\section{Questions types:}

- Qui parle le plus souvent, et le plus longtemps, en classe, les garçons ou les filles? Qui interrompt le plus souvent? Qui sont (éventuellement) les chefs de classe, des garçons ou des filles?

- Comment les enseignants défient ou renforcent-ils les modèles de comportement genrés?

- Certains élèves sont-il favorisés ou défavorisés en raison d'autres stéréotypes (d'ethnicité ou d'identité sexuelle, notamment)?

\section{Méthodes de recherche :}

Observez, si possible, les classes de différents instructeurs. Avant l'observation, décidez avec votre équipe comment vous allez «mesurer » et documenter ce que vous voyez. Il peut être utile d'élaborer un simple formulaire à remplir lors de l'observation. $\mathrm{Si}$ vous avez une montre, vous pouvez mesurer le temps de parole des filles et des garçons. 


\section{ÉQUIPE 3}

Comment les normes de genre affectent-elles l'accès aux programmes parascolaires et aux installations?

\section{Questions types:}

- Les garçons et les filles participent-ils à parts égales à toutes les activités parascolaires? Si non et si les activités sont différentes, lesquelles attirent plus les garçons? Et les filles?

- Dans l'ensemble, même s'ils participent à différentes activités, les garçons et les filles participent-ils de manière égale aux programmes parascolaires?

- L'école donne-t-elle un accès égal à ses installations (toilettes, salles de gym et terrains de sport), équipements, uniformes et fournitures aux activités plus féminines ou plus masculines?

- Pour les activités à participation mixte, qui assume généralement les rôles de leadership ?

\section{Méthodes de recherche:}

Dressez la liste de toutes les activités parascolaires (sports, clubs, gouvernement des élèves et autres). Observez chaque activité, interviewez les garçons et les filles qui y participent, et interviewez aussi l'instructeur-conseil du groupe.

\section{ÉQUIPE 4}

Quel est l'équilibre général de genre à l'école? Qu'en disent les statistiques?

\section{Questions types:}

- Les enseignants sont-ils principalement hommes ou femmes? Et la direction ? Quels enseignants (hommes ou femmes) ont les meilleures chances d'être promus aux postes de direction?

- Qui, des hommes ou des femmes, est plus vraisemblablement prof de math, de littérature, d'art?

- Qui enseigne dans les classes inférieures ? Et aux élèves plus âgés?

- Et les élèves ? Les filles et les garçons sont-ils inscrits à niveaux égaux ? Si non, pourquoi ?

- Les filles et les garçons s'inscrivent-ils à parts égales dans toutes les classes? Se sentiraient-ils tous également bienvenus dans toutes?

\section{Méthodes de recherche:}

La direction de votre école pourra sans doute vous procurer beaucoup de cette information par genre. Interrogez sinon autant d'enseignants que possible sur leurs classes et sur leurs collègues enseignant les mêmes branches. Si possible, obtenez aussi des données nationales sur la manière dont les normes de genre affectent les résultats scolaires, y compris les niveaux d'instruction atteints. 


\section{vente de stéréotypes : analyse de publicité}

APERC̣U : Les élèves créent un collage d'images publicitaires de femmes et d'hommes. Ils analysent les messages de genre et créent une publicité non stéréotypée.

OBJECTIFS : Aider les élèves à reconnaître et décrire le rôle de la presse écrite dans la création et le maintien des stéréotypes de genre; renforcer la réflexion critique.

\section{DURÉE :}

40 minutes

\section{MATÉRIEL :}

Tableau noir + craie ; magazines ou journaux avec publicité, ciseaux ; grandes feuilles de papier ; colle ou ruban adhésif.

\section{PRÉPARATION :}

Rassemblez suffisamment de magazines et journaux contenant des images publicitaires pour tout le groupe ou demandez aux élèves d'apporter des magazines qu'ils pourront découper.

\section{INSTRUCTIONS}

1 Distribuez les magazines et expliquez:

- Aujourd'hui nous allons réaliser des collages, au moyen d'images découpées dans des magazines et collées sur une feuille de papier.

- Vous allez chacun faire deux collages : images de femmes et images d'hommes.

2 Donnez aux élèves 10-15 minutes pour créer leurs deux collages, puis décrivez la suite :

- En commençant par le collage d'hommes, listez dans votre cahier les caractéristiques des hommes représentés dans les publicités.

- Faites-en de même pour les images de femmes.

3 Au tableau, tracez deux grands cercles intersectés. Marquez-en un «Hommes", l'autre «Femmes » et l'intersection « Les deux » et demandez:

- Quelles caractéristiques figurent seulement dans la publicité masculine? [Inscrivez-les dans le cercle «Hommes»].

- Et dans les images publicitaires de femmes? [Listez-les dans le cercle "Femmes»].
- Y en a-t-il qui figurent dans les deux? [Inscrivezles à l'intersection des deux cercles].

- Que remarquez-vous dans ce diagramme? Quelle est l'idée avancée au sujet des femmes ? Et des hommes?

- Comment ces images créent ou renforcent-elles les stéréotypes de genre? Quelles valeurs vendentelles aux femmes? Et aux hommes?

- Ces images reflètent-elles la réalité ?

- À quel point les images médiatiques deviennentelles des stéréotypes? Les médias en créent-ils de nouveaux ou reproduisent et renforcent-ils simplement ceux qui existent déjà ? Pourquoi ?

- Comment les jeunes sont-ils influencés par les images des médias? Avez-vous jamais douté de vous-mêmes après avoir regardé un magazine ou une publicité à la télé ? Que peut-on faire si on trouve une publicité blessante ou qu'on désapprouve ses images stéréotypées?

Devoir : Créez une publicité qui brise les stéréotypes de genre. [Si possible, invitez les élèves à afficher leur ceuvre ou à la présenter à la classe.] 


\section{l'espace-genre}

APERCQU : Les élèves tracent un plan de leur communauté et y indiquent les espaces sûrs et accessibles aux personnes de chaque sexe. Ils analysent l'effet des rôles de genre sur la participation à la vie civique. (Remarque : À l'instructeur de décider si cette activité convient ou non dans le contexte de sa communauté.)

OBJECTIFS : Apprendre aux élèves à analyser comment le genre affecte la mobilité, la sécurité et l'accès aux espaces communautaires; à citer au moins trois façons dont les normes de genre peuvent affecter la participation féminine à la vie civique ; renforcer la réflexion abstraite et les aptitudes à la représentation géographique de données.

\section{INSTRUCTIONS}

1 Expliquez aux élèves que vous allez dessiner une « carte de genre » de la communauté, en y indiquant les espaces extérieurs au foyer où se retrouvent principalement les hommes et les garçons (lieux sûrs de rencontre, jeux et autres activités), principalement les femmes et les filles, et les deux.

2 Répartissez les élèves en petits groupes (de même sexe ou non) et donnez à chacun une copie du plan général de votre communauté. (Pour les groupes avancés, prévoyez plus de temps et invitez-les à tracer leur propre plan.)

3 Donnez trois crayons (ou marqueurs) de couleur aux élèves et une liste des endroits suivants de la communauté. (Invitez les groupes avancés à dresser leur propre liste.)

\section{LISTE D'ENDROITS}

\section{Marchés}

Lieux de culte

École

Centre communautaire

Bibliothèque

Salle de jeux vidéo

Cinéma

Voie publique, le soir

Cafés ou salons de thé

Terrains de sport/basketball

Bars

Gare/terminus/aérogare

Mairie/bureaux municipaux

Lavoir (launderette, rivière)
DURÉE :

Étapes 1-5: 45 minutes (plus si les groupes doivent tracer leur propre plan et dresser leur propre liste d'endroits)

Étapes 6-7 : 45 minutes

\section{MATÉRIEL :}

Tableau noir + craie ; ruban adhésif ; 5 ou 6 copies d'un plan simple de la communauté tracé à l'avance (ou grandes

feuilles de papier sur lesquelles les élèves plus avancés peuvent tracer leur propre plan); crayons ou stylos de couleur.

\section{PRÉPARATION :}

Passez en revue la liste d'endroits et modifiez-la pour qu'elle reflète votre communauté. 
4 Décrivez le projet:

- Discutez brièvement avec le groupe si chaque emplacement est fréquenté principalement par les hommes et les garçons, les femmes et les filles ou les deux, et s'il est accueillant et sûr.

- Choisissez une couleur pour les endroits masculins, une autre pour les endroits féminins et une troisième pour les endroits mixtes, accueillants et sûrs pour les deux sexes. Marquez chaque emplacement sur la carte, dans la couleur choisie pour indiquer la nature « genrée » de l'espace.

- Cela fait, parlez de ce que votre plan dit de la mobilité, de la sécurité et de l'accès à l'espace public dans la communauté.

5 Circulez parmi les groupes tandis qu'ils préparent leurs cartes et animez leurs discussions.

6 Faites afficher les cartes au mur, puis invitez chaque groupe à présenter la sienne ou invitez les élèves à circuler et à observer chaque carte.

7 Posez les questions suivantes au groupe au complet (et inscrivez les réponses au tableau) :

- Quelles sont vos observations? Les filles peuventelles être aussi mobiles que les garçons? Qui a le plus accès à l'espace public?

- La sécurité en public est-elle la même pour les garçons et les filles?
- Ces conditions changent-elles quand les garçons grandissent ? Et les filles?

- Quelles sont les raisons souvent données pour expliquer ces différences?

- Que pensez-vous de la nature "genrée » de l'espace public?

- Pour participer activement à la vie civique, de quel accès à l'espace public sûr faut-il disposer?

- Dans quelle mesure l'accès limité des filles et des femmes à un espace sûr limite-t-il leur capacité d'exercer pleinement leur citoyenneté ? [Demandez aux élèves combien de femmes ils connaissent dans des fonctions publiques de décision? Par rapport aux hommes, combien détiennent un pouvoir public? Le gouvernement local est-il dirigé par des hommes ou des femmes?]

- Les garçons et les hommes sont-ils affectés de la même manière ? Pourquoi ou pourquoi pas?

Devoir : Choisissez un endroit où les femmes et les filles ne se sentent pas en sécurité et bienvenues. Décrivez ce qui devrait être différent pour que les choses changent. Comment procéder? 


\section{femmes d'action : projet livre d'enfants}

APERCU : Les élèves créent un livre d'enfants consacré à une femme illustre. (Remarque : Cette activité nécessite l'accès à une bibliothèque ou à Internet.)

OBJECTIFS: Aider les élèves à découvrir les femmes-leaders et à rapporter le courage et les accomplissements des femmes à leur propre vie ; renforcer les aptitudes de recherche.

\section{INSTRUCTIONS}

1 Distribuez la feuille d'accompagnement (ou copiez-la au tableau).

2 Divisez la classe en groupes de trois ou quatre élèves et expliquez:

- Aujourd'hui, nous allons commencer un projet qui passe par la découverte de femmes leaders de la justice sociale.

- Chaque groupe va faire des recherches sur la vie et l'œuvre d'une grande femme.

- Le groupe rédigera ensuite une histoire pour enfants au sujet de cette femme et en fera un livre relié. [Mentionnez les dispositions que vous avez prises pour présenter les livres aux enfants d'une école primaire proche.]

3 Passez en revue la liste de femmes proposée. [Expliquez comment le choix de chaque groupe sera opéré pour éviter les redoublements.] Demandez:

- Avez-vous entendu parler de ces femmes?

- Vous pouvez, avec mon accord, en proposer une autre pour le projet.
4 Passez en revue les questions-guides (sur la feuille distribuée ou à copier du tableau).

5 Expliquez: 1) si les groupes travailleront en classe ou à domicile ; 2) combien de temps ils ont pour achever le projet et vous le remettre; et 3 ) où ils peuvent rechercher l'information nécessaire.

6 Expliquez qu'après la phase de recherche, chaque membre du groupe devra assumer au moins un aspect de la réalisation du livre : rédaction ; révision ; illustrations ; couverture; reliure (couture, agrafage ou autre).

7 Consacrez le temps restant à la sélection de la « femme d'action » de chaque groupe et à la délégation des tâches (voir la feuille distribuée). Circulez parmi les groupes pour les aider.

8 Présentation des livres: Invitez chaque groupe à lire son livre au reste de la classe et, si possible, à de jeunes enfants. Demandez ensuite :

- Quelle femme admirez-vous particulièrement?

- Pensez-vous que vous pourriez jamais ressembler, par vos actions, à ces femmes?

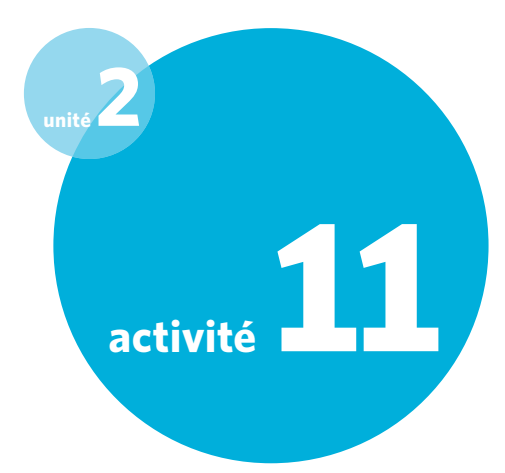

\section{DURÉE :}

Étapes 1-7: 45 minutes

Réalisation : 2 à 3 heures en classe ou comme devoir.

Étape $8: 45$ minutes

\section{MATÉRIEL :}

Papier ; marqueurs ; aiguille/fil, ruban adhésif, agrafeuse ou autre matériel ; « Feuille à distribuer aux élèves (instructions)»

\section{PRÉPARATION :}

Passez en revue la liste des femmes d'action proposée pour assurer leur pertinence dans votre contexte. Veillez à disposer d'une liste suffisamment longue pour que chaque groupe de trois ou quatre élèves puisse travailler sur une femme différente. Si possible, photocopiez la feuille pour vos élèves. Considérez d'organiser la présentation des livres finis dans une école primaire où vos élèves pourraient les lire à des enfants plus jeunes. 


\section{femmes d'action : projet livre d'enfants - instructions}

\section{FEMMES D'ACTION}

\section{Rigoberta Menchú}

Leader guatémaltèque des droits humains

\section{Rosa Parks}

Leader des droits civils aux ÉtatsUnis

\section{Mukhtaran Bibi}

Leader pakistanaise de la lutte contre la violence sexuelle

\section{Aung San Suu Kyi}

Leader politique au Myanmar

\section{Wangari Maathai}

Leader kenyane pour

l'environnement

\section{Michelle Bachelet}

Leader politique chilienne

\section{Arundhati Roy}

Auteur indienne

\section{Sophie Scholl ou Martha}

\section{Wertheimer}

Résistantes contre le nazisme

\section{Hillary Clinton}

Leader politique américaine

\section{Vera Ngassa ou Beatrice Ntuba} Juges camerounaises

\section{Louise Arbour}

Ancienne Haut-Commissaire aux droits humains de l'ONU

\section{Questions guides de recherche}

1 Quel est le nom de la femme sur laquelle vous effectuez votre recherche?

2 Recherchez un fait frappant ayant marqué le début de sa vie.

3 Quel problème social a-t-elle observé ? Comment l'a-t-elle remarqué ?

4 Comment savez-vous qu'elle se souciait de ce problème?

5 Qu'a-t-elle fait pour le résoudre?

6 Quil'a aidée?

7 Racontez une histoire au sujet d'un moment où elle a dû faire preuve d'une qualité personnelle que vous admirez : courage, persévérance, intégrité, patience, créativité, intelligence, bonnes relations avec les autres, vision du futur ou autre qualité.

8 Décrivez la différence qu'elle a faite dans le monde par ses actions.

9 Incluez, si possible, une citation qui lui est attribuée.

10 Donnez à votre livre un titre comportant, plus que le nom de votre héroïne, le thème qui lui est ou était cher, ou un autre point intéressant que vous avez découvert sur ses qualités personnelles.

Dans votre groupe, qui sera responsable de ...

la rédaction de l'histoire (peut être plusieurs personnes) :

la révision de l'histoire :

la relecture du texte :

l'illustration de l'histoire :

la conception et la création de la couverture :

la reliure du livre : 


\section{changer son corps}

APERCU : Les élèves identifient l'apparence féminine et masculine idéalisée dans leur culture et examinent les pratiques adoptées pour s'y conformer. Ils découvrent les conséquences de ces pratiques sur leur santé.

OBJECTIFS : Remettre en question la pression de conformité aux apparences « idéales » dictées par la culture ; décrire les pratiques, des femmes et des filles surtout, visant à atteindre cet idéal et les préjudices causés par ces pratiques ; renforcer la réflexion critique.

\section{INSTRUCTIONS}

1 Posez ces questions pour introduire le sujet:

- Quelles pressions pèsent sur les filles et les garçons d'avoir une certaine apparence?

- Ces apparences désirées sont-elles généralement réalistes ou idéalisées?

- Comment se sentent ceux non conformes à l'idéal ?

- Que fait-on (ou que fait-on faire à ses enfants) pour se conformer aux images idéalisées de la beauté ? Pensez aux cheveux, à la peau, à la forme du corps? [Écrivez les réponses au tableau.]

2 Ajoutez les pratiques dont vous désirez parler : Engraissement ou régime extrême Musculation extrême Bronzage ou blanchissement de la peau Maquillage, henné ou ongles vernis Perçage, scarification ou tatouage Rasage ou épilation Chirurgie esthétique Mutilation génitale des filles ou excision ${ }^{*}$

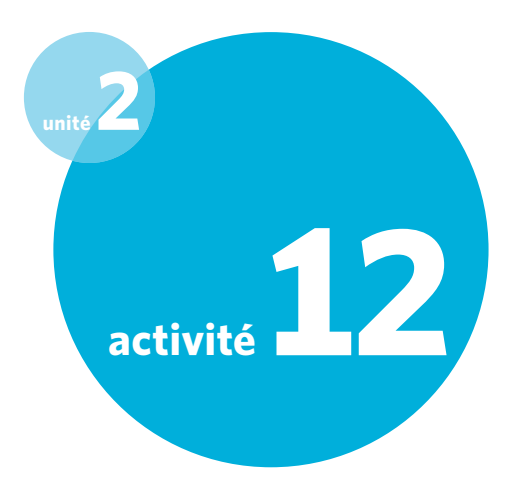

DURÉE :

45 minutes

\section{MATÉRIEL :}

Tableau noir + craie

3 Demandez:

- Que pensez-vous des pratiques listées? Lesquelles sont anodines, purement expressives, et lesquelles peuvent être nuisibles?

- Que penseraient les membres d'autres cultures des pratiques listées ici?

- D’où viennent ces idéaux ? La pression de se conformer à un type de corps idéalisé est-elle plus forte sur les femmes? Qui en profite et qui en souffre? Dans quelle mesure cette pression affecte-t-elle l'estime de soi ?

- Faut-il mettre fin aux pratiques nuisibles d'altération du corps ? Quelle pratique aimeriezvous voir disparaître?

- Quand vous étiez plus jeunes, vous a-t-on dit que cette pratique était dangereuse et devrait disparaitre?

- Avez-vous parlé à quelqu'un de ce que vous en savez et en pensez?

- Comment pourriez-vous le faire?

\section{PRÉPARATION :}

Pensez aux façons dont certains changent leur apparence, dans votre communauté. Quelles pratiques ont des conséquences sur la santé ? Passez en revue la liste proposée et considérez les pratiques que vous désirez discuter avec votre classe.

\footnotetext{
* La circoncision masculine est aussi une pratique courante qui implique l'altération du corps. Certains avantages de santé ont cependant été démontrés : elle protège partiellement l'homme contre certaines IST, y compris le VIH.
} 


\section{de la violence au respect dans les relations intimes}

APERCQU : Les élèves explorent le respect et la violence dans les relations intimes. Ils discutent comment favoriser le respect dans leurs propres relations.

OBJECTIFS: Aider les élèves à discuter les attitudes relatives à la violence dans les relations intimes et la promotion de relations basées sur le respect; renforcer l'expression créative écrite et art dramatique.

\section{DURÉE :}

90 minutes (l'activité peut être divisée en deux sessions)

\section{MATÉRIEL :}

Tableau noir + craie

\section{PRÉPARATION :}

Informez-vous sur la violence et réfléchissez-y dans votre contexte. N'oubliez pas qu'il s'agit d'un sujet difficile à aborder pour beaucoup car il touche à l'expérience personnelle ou à l'idée que la question doit rester privée. Veillez à ne jamais mettre vos élèves dans l'embarras.

\section{INSTRUCTIONS}

1 Présentez ce sujet comme très sérieux :

- Pour cette activité, nous allons discuter et analyser les différents types de violence qui affectent parfois les relations intimes.

- En un quart d'heure, vous allez préparer un petit sketch concernant une relation intime : couple marié, hétérosexuel non marié ou homosexuel.

- Le but est de nous aider à réfléchir sur la manière d'assurer une relation basée sur le respect.

2 Répartissez les élèves en quatre groupes (ou moins suivant le nombre total d'élèves) de cinq ou six membres chacun.

Dites à deux groupes: Préparez un petit sketch (moins de 5 minutes) présentant une relation intime empreinte de violence (physique ou psychologique). Essayez d'être réalistes, en prenant des exemples d'incidents dont vous avez été témoins ou dont vous avez entendu parler dans la communauté.
Dites aux deux autres groupes: Préparez un petit sketch (moins de 5 minutes) présentant une relation intime basée sur le respect mutuel. Montrez comment le couple résout un conflit ou une différence d'opinion sans recourir à la violence.

3 Circulez parmi les groupes tandis qu'ils préparent leur sketch et offrez-leur votre aide.

4 En commençant par les sketchs «violents », invitez chaque groupe à présenter son sketch à la classe. Après chaque sketch, demandez aux spectateurs s'ils ont des questions, puis invitez-les à identifier les caractéristiques individuelles ou d'une relation qui mènent à la violence.

5 Procédez de même pour les sketchs qui démontrent le respect, en identifiant cette fois les caractéristiques individuelles ou de la relation qui la rendent saine et non violente. 
6 Animez la discussion en posant les questions suivantes :

- Quelles sont les différentes formes de violence dans les relations intimes? [Sondez: contrôle, contrainte, éclats de voix, menace et violence physique.]

- Quelles sont les caractéristiques d'une relation violente? [Écrivez les réponses au tableau sous le titre "Relations violentes».]

- Pourquoi tant de personnes, surtout jeunes, se sentent-elles impuissantes face à la violence familiale ? Le sont-elles vraiment ?

- Quelles sont les caractéristiques d'une relation saine? Quels sont les ingrédients d'une relation basée sur le respect? [Écrivez les réponses au tableau sous le titre "Relations respectueuses ».]
- Les exemples présentés dans les sketchs sont-ils réalistes? Observez-vous de telles situations dans votre vie journalière ? Et les exemples de relations saines? En voyez-vous dans votre vie quotidienne?

- La violence physique est-elle principalement le fait des hommes à l'encontre des femmes, ou les femmes sont-elles tout aussi violentes envers les hommes? Quelles sont selon vous les causes de la violence intime? Quand vous en êtes témoin, que faites-vous généralement? Que pourriez-vous faire?

- Pensez-vous qu'il est possible d'avoir une relation intime basée sur le respect? Que pouvons-nous faire, chacun et chacune, pour avoir des relations intimes saines? 


\section{action contre la violence de genre}

APERCQU : En petits groupes, les élèves développent des stratégies contre la violence faite aux femmes et les présentent sous forme de journal télévisé.

OBJECTIFS : Apprendre aux élèves à énoncer au moins deux idées d'action contre la violence sexiste ; renforcer les aptitudes de résolution de problèmes et d'expression en public.

\section{DURÉE :}

Étapes 1-4:1 heure

Étapes 5-7:1 heure

\section{MATÉRIEL :}

Tableau noir + craie

\section{PRÉPARATION :}

Les élèves doivent être déjà informés sur la question de la violence faite aux femmes.

\section{INSTRUCTIONS}

1 Répartissez les élèves en groupes de quatre ou cinq et expliquez que la moitié va préparer un journal télévisé et l'autre, un exposé aux autorités gouvernementales.

2 Demandez : «À qui revient la responsabilité de mettre fin à la violence contre les femmes et les filles?»

\section{Expliquez:}

- Imaginez quelques stratégies ou programmes visant à réduire la violence faite aux femmes.

- Vous avez 40 minutes pour préparer une présentation de 5 à 10 minutes (un journal télévisé ou un exposé aux autorités locales ou nationales, suivant votre groupe).

- Partez de l'idée que votre public est déjà au courant du problème de la violence. Votre tâche est de présenter les mesures positives prises pour $\mathrm{y}$ faire face.
- Essayez d'inclure ce qui a été fait (ou peut être fait) par les jeunes et les adultes dans la vie de tous les jours; par les organisations locales et les communautés ; par l'État ou par l'ONU.

- Considérez aussi comment impliquer les hommes et les garçons dans cet effort.

Pour les exposés aux autorités locales/nationales, expliquez: Vous allez informer vos responsables gouvernementaux des mesures prises par certains groupes et individus. Précisez la réponse attendue du gouvernement. Certains élèves peuvent jouer le rôle de responsables gouvernementaux, d'autres celui d'experts ou de citoyens.

Pour le journal télévisé, expliquez: Votre tâche est d'attirer l'attention du public et d'être aussi brefs et clairs que possible. Présentez une histoire d'intérêt humain pour démontrer les efforts entrepris pour résoudre le problème de la violence dans les relations personnelles. Gardez votre présentation personnelle et engageante. 
4 Assurez-vous que tout le monde comprend bien la tâche. Donnez aux groupes 45 minutes de temps de préparation et circulez parmi eux pour les aider.

5 Avant les présentations, donnez aux groupes 5 minutes finales pour mettre la dernière touche à leur travail et à leur plan.

6 Invitez chaque groupe à présenter son travail. Prenez note des idées émises.

7 Après le dernier exposé, demandez :

- Vous a-t-il été difficile de trouver des moyens de réduire la violence faite aux femmes?

- Quels sont les thèmes ou idées communs avancés par les différents groupes pour aider les femmes?

- Et pour aider les hommes et les garçons?

- Quelles sont les idées qui vous paraissent les meilleures et pourquoi?

- La responsabilité de la lutte contre la violence faite aux femmes incombe-t-elle à tous?

- Y a-t-il quoi que ce soit que vous puissiez faire dès maintenant, en tant que jeunes, pour mettre fin à la violence faite aux femmes et aux filles au niveau de la famille, de l'école ou de la communauté? 


\section{images de normes et rôles de genre changeants}

APERCQU : Les élèves interviewent un grand-parent (ou une personne de cette génération) sur les rôles et normes de genre de leur jeunesse, les comparent à leur propre vécu et discutent de l'évolution des rôles de genre.

OBJECTIFS: Aider les élèves à citer au moins trois façons dont les rôles et normes de genre ont changé au fil du temps; renforcer les aptitudes de recherche.

\section{DURÉE :}

Étapes $1-3: 15$ minutes

Devoir : 1 heure

Étape $4: 30$ minutes

\section{MATÉRIEL :}

Tableau noir et craie (pour

l'étape 3); « Tableau

d'entrevue » à distribuer

\section{PRÉPARATION :}

Si certains élèves n'ont pas de personne plus âgée à interviewer, identifiez-en quelques-unes disposées à répondre à leurs questions. Si possible, faites une copie du tableau à distribuer pour chaque élève.

\section{INSTRUCTIONS}

1 Expliquez que cette activité explore l'évolution des normes de genre au fil du temps.

- Vous allez interviewer séparément deux personnes de la génération de vos grands-parents. Choisissez si possible quelqu'un du même sexe que vous. Si vous ne connaissez personne à interviewer, votre famille (ou moi-même) pourrons vous aider à trouver quelqu'un.

- Demandez-leur quels étaient les rôles des hommes et des femmes quand ils étaient adolescents et jeunes. Demandez-leur ce qui a changé depuis lors : en termes d'éducation, de loisirs, d'amitiés, d'amour, de coutumes matrimoniales et d'emploi. Laissez la personne s'exprimer librement sur ce qu'elle désire décrire.

2 Tracez le tableau d'entrevue au tableau ou, si vous en avez fait des copies, distribuez-les.

Expliquez:

- Dans la colonne de gauche, inscrivez les réponses de la personne à vos questions.

- Après l'entrevue, écrivez dans la colonne de droite ce qu'il en est de ces rôles aujourd'hui.
3 Assurez-vous que tout le monde comprend la tâche et a quelqu'un à interviewer. Indiquez les délais de chaque tâche (interview et tableau).

4 Après les entrevues, discutez:

- Qui avez-vous interviewé?

- Vous êtes-vous sentis à l'aise à interviewer des personnes plus âgées sur les rôles de genre? Y a-t-il eu des sujets difficiles à aborder?

- Qu'est-ce qui vous a surpris dans leurs réponses? Pensez-vous maintenant que les rôles de genre sont immuables ou qu'ils peuvent changer?

- À quels niveaux les rôles et les normes de genre ont-ils changé le plus ? Quels changements vous paraissent bons? Ou mauvais? Pourquoi ?

- Les facteurs du changement sont nombreux. Comment les médias mondialisés affectent-ils les rôles de genre? Et la technologie, et Internet ? Et les actes de leaders individuels, ou ceux des groupes pour la justice sociale?

- Pensez-vous que les rôles de genre vont continuer à changer ? Comment ? 


\section{TABLEAU D'ENTREVUE}

\begin{tabular}{|l|l|l|}
\hline \multicolumn{1}{|c|}{ Sujet } & Aux temps de vos grands-parents & Pour vous, aujourd'hui \\
\hline Comment c'était : & & \\
\hline L'école? & & \\
\hline Les tâches ménagères? & & \\
\hline Les loisirs avec les amis? & & \\
\hline Avoir une relation & & \\
amoureuse? & & \\
\hline Être parent? & & \\
\hline Le travail rémunéré ? & & \\
\hline Autres questions : & & \\
& & \\
\end{tabular}




\section{plaidoyer}

APERCQU : Les élèves composent une chanson ou écrivent un poème pour l'égalité de genre dans les relations. Avant cette activité, chaque groupe doit avoir achevé au moins l'une des activités suivantes de ce volume : 1, 3, 5, ou 6 .

OBJECTIFS : Apprendre aux élèves à analyser et identifier le pouvoir inégal dans les relations.

\section{DURÉE :}

Étapes 1-7: 45 minutes

Étape $8: 45$ minutes

\section{MATÉRIEL :}

Peut-être voudrez-vous montrer aux élèves quelques exemples de chansons, paroles de rap ou poèmes.

\section{PRÉPARATION :}

Familiarisez-vous avec le type de musique que vos élèves écoutent. Pour l'étape 4, considérez l'ajout de scénarios pertinents à votre contexte. Encouragez vos élèves à réfléchir à leurs propres scénarios et à vous les décrire. Choisissez peut-être de désigner quelques élèves comme juges (étape 6).

\section{INSTRUCTIONS}

1 Expliquez que cette activité porte sur la manière dont les rôles de genre mènent parfois à des différences de pouvoir dans nos relations.

Demandez :

- Qu'avons-nous appris jusqu'ici au sujet des rôles de genre et de l'égalité?

- Dans quelle mesure les rôles de genre peuventils affecter l'égalité de pouvoir dans les relations interpersonnelles? [Prévoyez 5 à 10 minutes de temps de réponse.]

2 Expliquez aux élèves qu'ils vont explorer le sujet à travers la composition de poèmes ou de chansons, dans le style hip-hop, rap ou autre.

3 Invitez les élèves à s'organiser par deux (même sexe ou fille-garçon) et à décider avec leur partenaire la répartition de la tâche : les deux composent et interprètent ensemble, ou l'un compose et l'autre interprète principalement.
4 Donnez quelques exemples de scénarios dont les élèves peuvent s'inspirer.

- Un garçon défend son ami homosexuel.

- Un homme dit à un ami qu'il n'a pas le droit de forcer sa femme à avoir des rapports sexuels.

- Un banquier explique à un mari récalcitrant pourquoi il accorde un prêt personnel à sa femme (ou la femme dit à son mari pourquoi elle a besoin de son propre argent et le mérite).

- Une adolescente en proie au harcèlement sexuel de garçons plus âgés.

- Un mari insiste que sa femme s'occupe seule du ménage et des enfants alors qu'ils travaillent tous les deux à temps plein.

- Une fille confrontée à l'opprobre après avoir été vendue por le travail sexuel.

- Une jeune femme punie par son mari pour la petitesse de sa dot.

- Un garçon ridiculisé par d'autres parce que son comportement manque de virilité.

- Une épouse ou une petite amie essaie de convaincre son mari ou partenaire d'utiliser le préservatif quand il le refuse. 
- Un garçon défend sa mère quand son père devient violent envers elle.

- Autre type d'idée : la déclaration de l'ancien Secrétaire général de l'ONU, M. Kofi Annan : « Nous savons comment changer la donne [du sida] ... Il faut un changement positif réel qui rende les femmes et les filles plus fortes et plus confiantes et qui transforme les relations entre les femmes et les hommes à tous les niveaux de la société. »

- Autres idées émises par les élèves mêmes, avec l'approbation de l'enseignant.

5 Donnez aux élèves ces directives :

- Prenez quelques minutes pour choisir un scénario avec votre partenaire.

- Consacrez le reste du temps de classe à composer votre chanson ou poème.

- Réfléchissez aux arguments des acteurs du scénario et à ce qu'ils pourraient dire pour renforcer leur position.

- Réfléchissez aussi à ce que pourrait ressentir la personne impliquée et à la manière dont elle voudrait mettre ses sentiments en paroles.

- N'oubliez pas que le sujet est sérieux et qu'il serait malvenu de prendre la violence à la légère.
6 Expliquez le moment où vos élèves présenteront le produit de leur travail. Si vous décidez de juger les œuvres, invitez trois ou quatre élèves (ceux timides à l'idée de se produire en public, peut-être) à sélectionner leurs trois œuvres préférées.

7 Donnez aux élèves 25 à 30 minutes de temps de composition. Au besoin, permettez-leur d'achever leur projet comme devoir.

8 Au moment des présentations (lors d'une autre session peut-être), clarifiez pour les juges les critères de sélection (meilleur message, meilleure performance, meilleure chanson ou poème) et lancez la représentation.

Devoir : Choisissez deux des questions suivantes et rédigez un paragraphe complet en réponse à chacune dans votre cahier.

- Qui a généralement le plus de pouvoir dans les relations intimes homme-femme?

- Quels sont les coûts du déséquilibre de pouvoir dans les relations?

- Le pouvoir peut-il aussi être inégal dans les relations homosexuelles?

- Comment atteindre une plus grande égalité dans les relations intimes. 


\section{débat sur l'éducation sexuelle}

activité

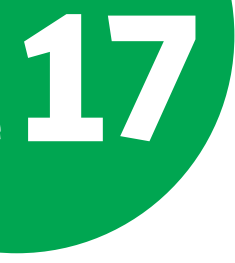

\section{DURÉE :}

45 minutes

\section{MATÉRIEL :}

Tableau noir + craie

\section{PRÉPARATION :}

Réfléchissez à la forme que prendra le débat. Lisez attentivement « Info enseignant » à la page suivante.

Si possible, invitez un autre enseignant à observer le débat et à servir de juge (déterminer les arguments gagnants). Si aucun autre enseignant n'est disponible, désignez deux élèves appelés à juger avec vous. Envisagez de remettre un prix à l'équipe gagnante.
APERC̣U : Les élèves débattent la question de savoir s'il faut informer les jeunes sur la sexualité, le genre et la santé sexuelle ou limiter l'éducation à l'abstinence jusqu'au mariage.

OBJECTIFS: Apprendre aux élèves à décrire les perspectives politiques de l'éducation sexuelle et à considérer leur propre point de vue ; renforcer l'esprit critique et l'expression en public.

\section{INSTRUCTIONS}

1 Présentez l'activité :

- On pense généralement à la sexualité comme à une question profondément personnelle et privée. Beaucoup d'aspects en sont pourtant à la table des débats de politique publique. Législateurs, politiciens et citoyens ordinaires disputent de l'éducation relative à la sexualité et au VIH.

- Nous allons aujourd'hui avoir notre propre débat sur l'éducation relative à la sexualité et au $\mathrm{VIH}$, avec préparation et présentation formelles d'arguments opposés.

2 Répartissez le groupe en deux équipes. Si vous n'avez pas de juge extérieur, invitez deux volontaires à juger le débat avec vous plutôt que d'y participer.

3 Présentez le titre du débat: «L'éducation sexuelle: l'école doit apprendre aux jeunes l'abstinence stricte jusqu'au mariage, sans parler du préservatif, de la contraception ou des relations de genre.»

4 Expliquez les procédures du débat:

- Une équipe va préparer les arguments en faveur de cet énoncé, et l'autre ceux contraires à cette position. Même si vous n'êtes pas d'accord avec les arguments avancés par l'équipe à laquelle vous avez été affectés, mettez-vous à la place d'une personne qui en est convaincue. Pensez à toutes les raisons qui appuient les arguments de votre côté du débat. Pensez aux données et arguments qui appuient l'autre côté. Couvrez tous les aspects de l'énoncé.

- Choisissez deux représentants appelés à parler pour votre équipe. [Un garçon et une fille pour les groupes mixtes.]

- Premiers intervenants : Vous allez chacun présenter un argument d'ouverture d'environ 3 minutes, tel que préparé par votre équipe. Seconds intervenants : Vous allez répondre à la présentation d'ouverture du côté opposé. Écoutez donc attentivement et prenez des notes pour vous aider à préparer votre réponse de 3 minutes.

- Nous aurons ensuite quelques minutes de questions-réponses entre les autres membres des équipes, qui pourront exprimer leurs commentaires ou poser leurs questions à l'équipe opposée, dont un membre non délégué pourra alors répondre. 
- Chaque équipe disposera de 10 minutes pour préparer ses arguments. Après le débat, le ou les juges désigneront l'équipe gagnante d'après, non pas leurs opinions personnelles, mais plutôt l'efficacité de la préparation et des arguments présentés.

5 Annoncez l'équipe chargée de défendre l'énoncé et celle appelée à s'y opposer. Donnez à chacune 10 minutes pour préparer leurs remarques. Circulez pour les aider et assurer la participation de tous. Si vous avez des élèves-juges, informez-les de leur tâche de chronométrer la durée des présentations et d'indiquer aux intervenants la fin de leur temps de parole. Guidez-les aussi sur la manière de juger les débats (en privilégiant par exemple les arguments factuels solides).

6 Invitez le premier intervenant de chaque équipe à présenter son argument d'ouverture (3 minutes chacun, pour un total de 6 minutes). Invitez ensuite le second intervenant de chaque équipe à répondre à l'argument de l'équipe opposée (3 minutes chacun, pour 6 au total). Enfin, invitez les commentaires et questions des autres membres des équipes ( 6 minutes). Indiquez le moment venu d'un dernier bref commentaire de chaque équipe.

7 Consultez le ou les juges et déterminez l'équipe gagnante. Avec les élèves-juges, offrez une critique concrète positive à chaque groupe sur les qualités de sa présentation.
8 Après l'annonce du gagnant, discutez :

- Combien d'entre vous ont été affectés à un côté conforme à votre opinion? [Mains levées] Contraire à vos opinions? [Mains levées] Vous a-t-il été facile ou difficile de défendre des arguments contraires à ce que vous pensez?

- L'éducation sexuelle est-elle le seul aspect de la sexualité devenu affaire de politique publique? Ou bien la société exerce-t-elle un certain contrôle sur beaucoup de ses aspects? Concernant quelles questions touchant à la sexualité y a-t-il des lois, des coutumes et des normes sociales qui régissent les pensées et les actes? [Sondez: rapports sexuels forcés; normes de comportement sexuel différentes suivant le sexe; couverture totale du corps des femmes en public (dans certains contextes); pressions sur les garçons pour qu'ils deviennent sexuellement actifs; opprobre (ou criminalisation) de l'homosexualité.]

- Les attitudes culturelles dominantes relatives à la sexualité rencontrent-elles l'accord de tous? Certains adoptent-ils d'autres attitudes de vie? Les attitudes relatives à la sexualité sont-elles les mêmes partout? Restent-elles toujours les mêmes ou évoluent-elles? La sexualité est-elle donc une question strictement privée, ou est-ce aussi une question d'intérêt social au sens large?

\section{INFO ENSEIGNANT}

\section{L'école doit apprendre}

l'abstinence sexuelle stricte

jusqu'au mariage, sans parler du

préservatif, de la contraception

ou des relations de genre. OUI ou NON?

Dans beaucoup de pays, le débat fait rage sur l'éducation sexuelle. Ceux qui pensent que les jeunes ne doivent être informés que de l'abstinence sexuelle croient souvent que leur sensibilisation aux pratiques sexuelles sûres favorise leur accès précoce à l'activité sexuelle. La recherche démontre pourtant qu'une éducation complète à la sexualité n'accélère nullement l'accès aux premiers rapports. Ceux qui croient en revanche en l'éducation complète à la sexualité (y compris aux pratiques sexuelles sûres) estiment qu'elle donne aux jeunes les outils nécessaires à la prise de décisions saines et sûres concernant leur vie sexuelle. 


\section{premières impressions}

activité

\section{8}

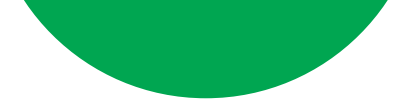

\section{DURÉE :}

40 minutes

\section{MATÉRIEL :}

Tableau noir + craie ; copies de «L'histoire de Maya » pour la moitié des élèves et de « L'histoire de Rasul » pour l'autre moitié ; espace suffisant pour que les deux groupes puissent se parler sans s'entendre l'un l'autre.

\section{PRÉPARATION :}

Soyez prêt à expliquer ce qu'est un adjectif. Comment allez vous assurer le maintien d'une discussion respectueuse? Soyez prêt à confronter l'usage d'un langage irrévérencieux, concernant les filles sexuellement actives surtout. Lisez l'information relative aux normes différenciées suivant le sexe à l'unité 3 du GUIDE (accessible sur $<$ www.unseulprogramme. org>) ou référez-vous à votre propre programme.
APERCQU : Les élèves examinent leur réaction à une histoire suivant qu'ils pensent que le personnage principal est une fille ou un garçon.

OBJECTIFS: Sensibiliser les élèves aux hypothèses et jugements que l'on pose souvent au sujet d'une personne suivant son sexe ; renforcer la réflexion critique.

\section{INSTRUCTIONS}

1 Expliquez:

- Aujourd'hui, nous allons lire et discuter une histoire qui relate le vécu, les sentiments et les défis que rencontrent les adolescents.

- Nous allons répartir la classe en deux groupes, qui liront chacun une histoire, discuteront leurs opinions et répondront à quelques questions.

2 Divisez la classe en deux. Distribuez « L'histoire de Maya » à un groupe et "L'histoire de Rasul » à l'autre. (Ne dites rien des histoires). Donnez aux groupes le temps de lire leur histoire, de répondre aux questions et de comparer les réponses de chacun au sein du groupe. Veillez à ce que les deux groupes ne s'entendent pas l'un l'autre.

3 Au bout de 15 minutes, invitez le groupe « Rasul » à partager brièvement ses réponses aux questions posées à la fin de l'histoire, sans expliquer ni discuter son raisonnement. Inscrivez les réponses clés (adjectifs, étiquettes) au tableau.
4 Invitez de même le groupe qui a lu l'histoire de Maya.

5 Intitulez la liste de mots produite par le premier groupe « Rasul », et celle produite par le second groupe, « Maya ».

6 Révélez que les deux histoires sont identiques, à l'exception du sexe de la personne. Demandez :

- Que remarquez-vous dans les réponses données pour Maya, par rapport à celles données pour Rasul ?

- Que nous disent ces réponses sur les normes applicables aux filles, par rapport aux garçons?

- Cette double mesure affecte-t-elle les filles de votre communauté ?

- Que pensez-vous de cette double mesure? 


\section{L'histoire de MAYA}

Maya a eu ses premiers rapports sexuels à l'âge de 16 ans. Ses deux amies la taquinaient parfois parce qu'elle n'avait jamais eu de petit ami. Elles l'ont encouragée à sortir avec un garçon et à avoir des rapports avec lui. Elle était curieuse et, sous la pression de ses amies surtout, elle a abordé le garçon et ils ont eu des rapports sexuels. Presque tout le monde, à l'école, a appris que Maya et le garçon avaient couché ensemble.

Les deux années suivantes, Maya a eu des rapports avec quatre autres garçons : un camarade de classe, un voisin, un garçon rencontré au travail et un ami d'une amie. Elle trouvait du plaisir à avoir des rapports sexuels. Maya savait qu'elle ne voudrait épouser aucun de ces garçons, mais elle les aimait bien et ne leur a jamais menti au sujet de ses sentiments. Avoir des partenaires sexuels donnait à Maya le sentiment d'être séduisante et de compter.

La plupart du temps, Maya faisait bien attention à utiliser le préservatif, mais elle s'est une fois retrouvée enceinte et s'est fait avorter. Elle a aussi contracté une infection sexuellement transmissible mais s'est fait soigner tout de suite.

Maya est aujourd'hui fiancée à un jeune homme que sa famille veut la voir épouser. Ce jeune homme lui plaît, mais elle sait qu'elle n'est pas censée avoir de rapports sexuels avec lui avant leur mariage dans deux ans. Lors d'une visite chez sa cousine dans une autre ville, elle a rencontré un de ses anciens petits amis qui lui a demandé de lui faire l'amour. Maya a décidé que personne ne souffrirait de cette escapade avant son mariage. Elle s'est dit que deux ans sans sexe, c'était bien long. Elle s'est ensuite confiée à sa meilleure amie, qui s'est montrée compréhensive.

\section{Après avoir lu ce récit, réfléchissez aux questions suivantes et discutez-les avec votre groupe :}

1 D'après vous, comment Maya est-elle vue par ses pairs ? Quelle « étiquette » lui donnent-ils ?

\section{Que pensez-vous de Maya?}

3 Dressez une liste d'au moins trois ou quatre adjectifs qui décrivent Maya. Par exemple, est-elle heureuse ou malheureuse? Sûre d'elle ou mal dans sa peau ? Honnête ou malhonnête ? Réaliste ou inconsciente ? Belle ou laide? Respectable ou méprisable? Une fille typique ou atypique ? Vertueuse ou immorale? 


\section{L'histoire de RASUL}

Rasul a eu ses premiers rapports sexuels à l'âge de 16 ans. Ses deux amis le taquinaient parfois parce qu'il n'avait jamais eu de petite amie. Ils l'ont encouragé à sortir avec une fille et à avoir des rapports avec elle. Il était curieux et, sous la pression de ses amis surtout, il a abordé la fille et ils ont eu des rapports sexuels. Presque tout le monde, à l'école, a appris que Rasul et la fille avaient couché ensemble.

Les deux années suivantes, Rasul a eu des rapports avec quatre autres filles : une camarade de classe, une voisine, une fille rencontrée au travail et une amie d'un ami. Il trouvait du plaisir à avoir des rapports sexuels. Rasul savait qu'il ne voudrait épouser aucune de ces filles, mais il les aimait bien et ne leur a jamais menti au sujet de ses sentiments. Avoir des partenaires sexuelles donnait à Rasul le sentiment d'être séduisant et de compter.

La plupart du temps, Rasul faisait bien attention à utiliser le préservatif, mais une fille s'est retrouvée enceinte et s'est fait avorter. Il a aussi contracté une infection sexuellement transmissible mais s'est fait soigner tout de suite.

Rasul est aujourd'hui fiancé à une jeune femme que sa famille veut le voir épouser. Cette jeune femme lui plaît, mais il sait qu'il n'est pas censé avoir de rapports sexuels avec elle avant leur mariage dans deux ans. Lors d'une visite chez son cousin dans une autre ville, il a rencontré une de ses anciennes petites amies qui lui a demandé de lui faire l'amour. Rasul a décidé que personne ne souffrirait de cette escapade avant son mariage. Il s'est dit que deux ans sans sexe, c'était bien long. Il s'est ensuite confié à son meilleur ami, qui s'est montré compréhensif.

\section{Après avoir lu ce récit, réfléchissez aux questions suivantes et discutez-les avec votre groupe :}

1 D'après vous, comment Rasul est-il vu par ses pairs ? Quelle « étiquette » lui donnent-ils ?

\section{Que pensez-vous de Rasul?}

3 Dressez une liste d'au moins trois ou quatre adjectifs qui décrivent Rasul. Par exemple, est-il heureux ou malheureux? Sûr de lui ou mal dans sa peau ? Honnête ou malhonnête ? Réaliste ou inconscient ? Beau ou laid ? Respectable ou méprisable? Un garçon typique ou atypique? Vertueux ou immoral ? 


\section{la vérité sur le désir}

APERCU : Les élèves répondent à un questionnaire « vrai ou faux » sur le désir sexuel et le genre.

OBJECTIFS : Apprendre aux élèves à distinguer le vrai du faux sur le désir sexuel.

\section{INSTRUCTIONS}

1 Dites aux élèves:

- Nous allons parler aujourd'hui de la question du désir sexuel et dissiper quelques mythes courants à son sujet.

- Nous allons commencer par un exercice « vrai ou faux ».

2 Distribuez la feuille d'exercice et demandez aux élèves d'y répondre rapidement.

3 Invitez un volontaire à lire le premier énoncé et encouragez la discussion en demandant :

- Combien d'entre vous pensent que cet énoncé est vrai?

- Qui pense qu'il est faux?

Si personne ne répond correctement, posez des questions qui aident le groupe à arriver à la bonne réponse. Ne la révélez vous-même que si personne ne la propose. Ne manquez pas de demander s'il reste des questions ou commentaires à émettre.
4 Répétez l’approche pour chaque énoncé.

5 Consacrez les 10 dernières minutes aux questions suivantes :

- Y a-t-il des choses que vous croyiez vraies au début de la classe et qui se sont avérées être des mythes?

- Quel est l'effet de cette désinformation sur les sentiments qu'on a de soi et de sa sexualité ?

- Que remarquez-vous dans les messages de la société sur le désir masculin, par rapport au désir féminin?

- Quel effet ce genre de mythes répandus peut-il avoir, selon vous, sur les relations intimes?

- Pourquoi pensez-vous que nous recevons des messages différents sur le désir masculin et le désir féminin ? Est-ce justifié? activité

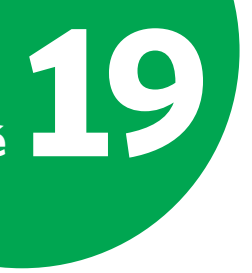

\section{DURÉE :}

45 minutes

\section{MATÉRIEL :}

Tableau noir + craie ; une copie de la feuille à distribuer aux élèves « le désir sexuel »

(ou copie des questions au tableau); « Clé pour

l'enseignant ».

\section{PRÉPARATION :}

Lisez et, au besoin, adaptez la feuille d'exercice. Veillez à disposer de l'information nécessaire à la clarification des réponses. 


\section{FEUILLE À DISTRIBUER AUX ÉLÈVES}

\section{LE DÉSIR SEXUEL : VRAI OU FAUX?}

Lisez chaque énoncé. Inscrivez dans la case de droite s'il vous paraît vrai (V) ou faux (F).

V ou F?

\begin{tabular}{|c|c|}
\hline 1 & La plupart des femmes ne désirent pas vraiment les rapports sexuels. \\
\hline 2 & Quand on désire des rapports sexuels, il faut en avoir. \\
\hline 3 & L'attente sociale qui veut que les femmes ne désirent pas les rapports sexuels peut affecter leur niveau de désir. \\
\hline 4 & Si un garçon a une érection, c'est qu'il désire des rapports sexuels. Même chose pour une fille dont le vagin se lubrifie. \\
\hline 5 & Certaines femmes désirent des rapports sexuels plus que leur partenaire n'en désire. \\
\hline 6 & On peut avoir des fantasmes sexuels sans désirer les vivre dans la réalité. \\
\hline 7 & La peur d'une grossesse ou d'une IST peut affecter le désir sexuel. \\
\hline 8 & Tout le monde connaît un désir sexuel changeant dans le temps et suivant les circonstances. \\
\hline 9 & Les hommes désirent toujours avoir des rapports sexuels. \\
\hline 10 & La plupart des personnes âgées ressentent encore un désir sexuel. \\
\hline 11 & Un homme qui n'a pas d'érection dans une situation sexuelle ne désire pas la personne avec laquelle il se trouve. \\
\hline 12 & Certains médicaments affectent le désir sexuel. \\
\hline 13 & Une personne peut ressentir un désir sexuel pour une autre pour laquelle elle n'éprouve pas d'amour. \\
\hline 14 & Il est parfois normal pour les hommes de ne pas désirer de rapports sexuels. \\
\hline 15 & Une personne qui désire des rapports sexuels devient sexuellement excitée. \\
\hline
\end{tabular}




\section{réponses à la feuille d'exercice « le désir sexuel : vrai ou faux? »}

1 Faux La plupart des femmes désirent les rapports sexuels, mais si elles n'y trouvent pas de plaisir, elles peuvent s'en désintéresser.

2 Faux Le désir sexuel ne doit pas mener à l'activité sexuelle. En fait, on peut aimer le sentiment d'excitation sans désirer passer à l'acte.

3 Vrai Les attentes sociales affectent le désir sexuel. Si une femme pense qu'elle ne doit pas désirer les rapports sexuels, elle peut supprimer ou nier son désir.

4 Faux L'érection et la lubrification peuvent se produire sans raison ou pour des raisons autres que le désir. Par exemple,la plupart des hommes ont une érection le matin parce que leur vessie est pleine. Les adolescents ont souvent des érections spontanées sans désir ni excitation sexuelle.

5 Vrai Le niveau de désir varie suivant la personne et les circonstances. Dans certains couples, à certains moments de la vie, la femme peut avoir un désir sexuel supérieur à celui de son partenaire.

6 Vrai Les fantasmes ne sont souvent ... que des fantasmes, que la personne ne désire pas réaliser.

7 Vrai Les états affectifs, de peur par exemple, peuvent affecter le désir sexuel d'une personne.

8 Vrai Les niveaux « normaux » de désir sexuel varient et changent suivant différents facteurs, de nature physique, affective, sociale ou autre. Si le niveau de désir sexuel d'une personne est problématique, cette personne peut consulter un professionnel de la santé sexuelle.

9 Faux Les hommes ne désirent pas toujours avoir des rapports sexuels. Leur niveau de désir varie, comme pour tout le monde.

10 Vrai Le désir sexuel peut diminuer avec l'âge. Si la société accepte mal la sexualité des personnes âgées, l'opprobre peut affecter leur désir. Beaucoup de personnes ressentent un certain désir sexuel tout au long de leur vie.

11 Faux Un homme peut ne pas avoir d'érection dans une situation sexuelle pour de nombreuses raisons, y compris d'ordre médical (diabète ou maladie cardiaque, par exemple), médicamenteux ou affectif (anxiété relative à sa performance sexuelle, colère ou simple préoccupation).

12 Vrai L'amoindrissement du désir sexuel peut être un effet secondaire de certains médicaments. D'autres, comme le Viagra, accroissent la fonction érectile et sont pris précisément dans ce but. Les médecins omettent souvent de parler de ces effets à leurs patients. Rien n'empêche de poser la question à un médecin ou à un pharmacien.

13 Vrai On confond souvent le désir sexuel et l'amour. On peut aimer quelqu'un sans désirer avoir de rapports sexuels avec lui ou elle, ou bien désirer avoir des rapports sexuels avec quelqu'un qu'on n'aime pas nécessairement.

14 Vrai Personne, homme ou femme, ne désire toujours avoir des rapports sexuels.

15 Faux On désire parfois avoir des rapports sexuels mais l'excitation ne vient pas. Cela peut arriver à tout le monde. II n'y a pas lieu de s'en inquiéter. 


\section{se sentir différent}

\section{DURÉE :}

35-40 minutes

\section{MATÉRIEL :}

Tableau noir + craie

\section{PRÉPARATION :}

Lisez la description complète de l'activité. Réfléchissez à la manière d'aborder la question des minorités sociales avec sensibilité et respect.
APERC̣U : Les élèves identifient les sentiments d'appartenance ou de différence par rapport aux autres et réaffirment les droits humains des minorités, même sexuelles.

OBJECTIFS : Apprendre aux élèves à identifier les émotions qu'ils associent au fait d'être différents des autres; réaffirmer les droits humains de tous, y compris les minorités sexuelles; renforcer la réflexion affective et les aptitudes au dialogue.

\section{INSTRUCTIONS}

1 Présentez le sujet :

- Réfléchissez à deux choses importantes (autres que votre âge) qui vous rendent fort semblables à la plupart de vos camarades réunis ici.

- Pensez maintenant à deux choses qui vous différencient le plus des autres. Vous n'aurez pas à révéler ces choses.

- Quels sentiments évoquent en vous les deux choses qui vous rapprochent des autres? Écrivez dans votre cahier quatre mots d'émotion qui décrivent ces sentiments.

- Revenez maintenant à vos différences. Quels sont les sentiments ou émotions qui y sont associés? Écrivez quatre mots qui les décrivent.

2 Animez la discussion en posant ces questions :

- Sans rien dire de ce qui vous rend semblables ou différents, quels sont les sentiments que vous associez à la similarité avec les autres? [Écrivez les réponses dans une colonne au tableau.]

- Quelles émotions associe-t-on au sentiment d'être différent? [Écrivez-les dans une deuxième colonne.]
3 Rappelez au groupe que tout le monde se sent différent, "pas comme les autres ", et que ce sentiment est parfois normal. Consacrez au moins 15 minutes aux questions suivantes :

- Quand la différence est-elle une bonne chose? Quels sont les avantages d'être unique?

- Quand la différence est-elle plus négative?

- Comment les personnes jugées « différentes » sontelles traitées? Qu'en pensez-vous? [Sondez: Ce traitement est-il juste?]

- Comment voulez-vous être traités quand vous vous sentez différents? Les personnes différentes (qui ne font de mal à personne) ont-elles les mêmes droits qu'autrui ? Leur différence importe-t-elle?

Devoir : Pensez à un groupe social minoritaire. [Sondez : handicapés, castes inférieures, minorités ethniques ou raciales, immigrants, minorités sexuelles.] Comment ces groupes sont-ils traités dans votre communauté ? Leurs droits sont-ils parfois violés ? Peutil arriver qu'un membre de ces groupes s'élève pour défendre leurs droits? 


\section{attirance vers une personne du même sexe : histoires vraies}

APERC̣U : Les élèves lisent le récit d'adolescents qui se rendent compte qu'ils sont homosexuels et discutent leurs réactions. L'information incorrecte est rectifiée.

OBJECTIFS : Aider les élèves à décrire deux exemples fréquents du vécu de jeunes qui sont attirés par d'autres du même sexe, à les comprendre et à rectifier l'information incorrecte; renforcer la réflexion critique et l'aptitude à paraphraser.

\section{INSTRUCTIONS}

1 Divisez la classe en trois groupes. Expliquez:

- Nous allons aujourd'hui parler du vécu social et des sentiments de jeunes homosexuels, ce qui veut dire qu'ils sont attirés exclusivement ou principalement vers d'autres du même sexe. Vous allez lire trois histoires vraies.

- Chaque groupe reçoit une histoire différente. Vous allez discuter et répondre par écrit à trois questions.

2 Écrivez les questions suivantes au tableau et lisezles à haute voix :

Quels sont les sentiments évoqués par l'auteur de chaque récit? Listez tous ceux que vous percevez. Que pense l'auteur de chaque récit des homosexuels? Comment l'auteur de chaque récit réagit-il ou elle à ses sentiments?

3 Distribuez les feuilles. Donnez aux groupes 10 minutes pour lire, discuter et rédiger leur réponse aux questions. Dites-leur qu'ils partageront leurs réponses avec le groupe au complet.

4 Au bout de 10 minutes, demandez à deux élèves du groupe Nia de paraphraser (pas lire) brièvement l'histoire de Nia et de présenter leurs réponses aux trois questions. Écrivez les sentiments de Nia au tableau. Répétez pour Lee et pour Mo.

5 Réservez 15 minutes à la discussion des questions suivantes avec le groupe au complet :

- Dans l'ensemble, quels types de sentiments Nia, Lee et Mo ressentent-ils envers leur homosexualité ? Pourquoi ont-ils ces sentiments? Comment ont-ils réalisé qu'ils étaient gay ou lesbienne? Leur est-il arrivé quelque chose qui les a rendus homosexuels?

- Qu'avez-vous pensé à la lecture de ces récits? Qu'est-ce qui vous a surpris? Qu'avez-vous appris sur les homosexuels?

- Les attitudes et les idées relatives à la diversité sexuelle sont-elles en train de changer?

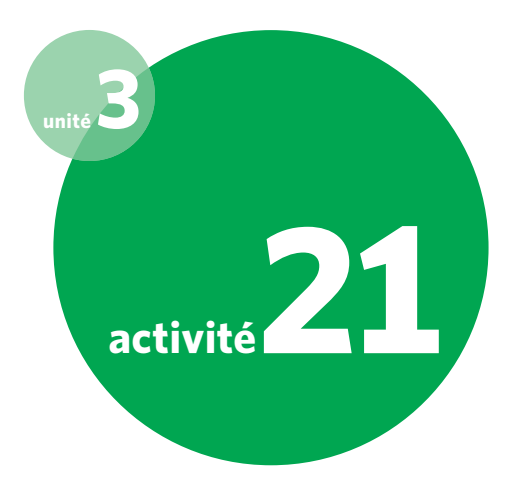

DURÉE :

45-50 minutes

\section{MATÉRIEL :}

Tableau noir + craie ; copies de la feuille à distribuer aux élèves « études de cas - grandir gay ».

\section{PRÉPARATION :}

Préparez-vous bien pour pouvoir répondre aux questions posées sur les récits ou le sujet. Veillez à ce que vos élèves abordent sérieusement le sujet et soyez sûr de vous sentir à l'aise à l'idée d'en parler. Au besoin, consultez une organisation experte sur la question de la diversité sexuelle ou une autre source fiable. Si les termes employés ici (« homosexuel», « gay » et « lesbienne ») ont des connotations négatives dans votre contexte, choisissez-en d'autres moins négatifs. 


\section{études de cas : grandir gay}

\section{GROUPE 1 - L'histoire de Nia :}

J'ai 25 ans et je suis lesbienne. Enfant, j'étais bonne élève et j'avais beaucoup d'amis. À la puberté, j'ai commencé à me sentir différente. Dans mes rêves, j'embrassais quelqu'un dont je ne voyais pas le visage mais dont je sentais le corps, féminin. Déconcertée, je me suis mise à me haïr. À 15 ans, j'ai soudain découvert que j'étais vraiment attirée par une fille plus âgée que je connaissais : un vrai béguin. Cela ajouté à mes rêves, j'ai compris et admis que j'étais attirée par les filles. Or ma première rencontre du mot « homosexuelle » a été dans un article de magazine parlant du viol d'une fille de 17 ans par une policière. Je me souviens de cette façon dont l'homosexualité était décrite comme une perversion et une maladie. Il y avait un autre article sur des hommes " gay » et le sida. Les deux récits dépeignaient les homosexuels comme pervers et malades. J'ai compris que je serais paria, l'une de « ces gens-là ». Tout le monde veut être aimé et faire partie intégrante de la société personne ne veut en être exclu. Je pensais que quelque chose n'allait pas en moi, que j'étais malade. Le mot «lesbienne » est devenu profondément menaçant pour moi. C'était un mot tabou ; j'étais un sujet tabou.

J'avais peur. Je me voyais bizarre et anormale. Pour faire face, je me suis repliée sur mes études et je n’ai plus été vue que comme un rat de bibliothèque.

\section{GROUPE 2 - L'histoire de Lee :}

J'ai 23 ans et je suis gay. J'ai grandi à la campagne. Vers l'âge de 11 ans, j'ai commencé à remarquer que j'aimais voir le corps des hommes. J'étais surpris et je ne comprenais pas. Il y avait un homme, où j'habitais, qui était très efféminé. On l'avait surnommé « la fille». J'avais peur de lui et je ne voulais pas être comme lui. Je me souviens aussi d'un magazine de sexe qui m'était tombé sous la main, dans lequel on parlait de " gays". Je l'ai lu et j'en ai été effrayé. Ma famille a quitté la campagne pour la ville, où mes amis parlaient de vouloir coucher avec des filles. Je mentais et je disais que je m'intéressais à une fille en particulier, alors que je ne pensais qu'à des hommes. Pour me cacher, je me montrais plus dur que les autres gars. Je me suis mis à faire du sport, dans l'espoir que personne ne découvre mon secret. Mais je me haïssais, à ne pas comprendre pourquoi j'étais ainsi. J'ai décidé d'essayer, je suis sorti avec cinq filles. Je leur ai offert des cadeaux, je les ai emmenées au ciné. J'essayais, mais je n'y arrivais pas. J'ai été jusqu'à coucher quatre fois avec une fille, mais sans y trouver la moindre satisfaction. 


\section{études de cas : grandir gay}

\section{GROUPE 3 - L'histoire de Mo :}

J'ai 22 ans et je suis gay. Enfant, j'avais des voisins homosexuels et mes parents avaient d'autres amis gay. J'ai donc grandi en sachant que les homosexuels sont des gens comme les autres - comme mes parents, ils avaient des enfants, un boulot, des chats et des chiens. À 15 ans, je me suis rendu compte que j'aimais mon copain Benno plus que comme un ami. Je ne savais pas comment lui en parler, alors j'ai demandé à l'ami gay de ma mère, qui vivait avec son partenaire depuis 20 ans. Il m'a d'abord dit qu'il savait combien il fallait de réflexion honnête pour découvrir qui nous sommes. Puis il m’a demandé comment était Benno et m’a encouragé à être honnête avec lui. Il m’a expliqué que Benno ne partagerait peut-être pas mes sentiments et qu'en fait, il pourrait même prendre ses distances. Le lendemain, après l'entraînement de foot, j'ai parlé à Benno. Il s'est d'abord fâché et m'a dit qu'il ne pensait pas que nous pourrions continuer à être amis. J'étais bouleversé. Mais quelques jours plus tard, Benno est venu chez moi. Il m'a dit qu'il n'éprouvait aucun sentiment sexuel envers moi ou envers aucun autre garçon, mais qu'il voulait que nous restions amis.

J'étais si heureux d'avoir retrouvé mon copain, même s'il n'y aurait jamais d'idylle entre nous. Il n'a pas toujours été facile d'être adolescent gay et j'ai souvent rencontré des gens que mon mode de vie mettait mal à l'aise. Je sais que j'ai beaucoup de chance d'avoir le soutien de ma famille. Beaucoup de jeunes n'ont pas cet avantage. C'est pour ça que je travaille pour un service à la disposition des jeunes qui se posent des questions sur leur sexualité et n'ont aucun autre soutien. 


\title{
comportement sexuel - les mythes et les faits
}

\author{
APERCQU : Les élèves participent à un jeu dans lequel ils décident si les énoncés qui \\ leur sont soumis sur le comportement sexuel sont vrais ou faux. Les idées fausses sont \\ corrigées et discutées.
}

OBJECTIFS : Aider les élèves à distinguer le vrai du faux sur le comportement sexuel.

\section{DURÉE :}

Étapes 1-4: 45-50 minutes

Étapes 5-6 (si un questionsréponses de suivi est prévu) : 10 minutes

\section{MATÉRIEL :}

Tableau noir + craie ; une copie de la feuille à distribuer aux élèves « comportement sexuel » pour chaque équipe ; bandelettes de papier et (si l'étape 6 est prévue) un sac ou une grande enveloppe ; clé de réponses.

\section{PRÉPARATION :}

Lisez les énoncés présentés sur la feuille à distribuer et veillez à pouvoir expliquer pourquoi ils sont vrais ou faux. Décidez d'inclure ou non les étapes 5 et 6 . Si oui, prévoyez une source d'information sûre pour répondre aux questions écrites.

\section{INSTRUCTIONS}

1 Dites aux élèves qu'ils vont discuter les mythes du comportement sexuel et distinguer le mythe de la réalité.

2 Divisez la classe en deux équipes. Au tableau, tracez un tableau de marque (une colonne par équipe). Distribuez les feuilles et expliquez :

- Par équipe, vous allez discuter chaque énoncé et déterminer s'il est vrai ou faux. Votre équipe a 7 minutes pour remplir la feuille.

- Cela fait, nous passerons en revue toutes les questions et chaque équipe donnera sa réponse. Chaque équipe recevra un point par réponse correcte. [Lancez l'activité - 7 minutes]

3 Invitez un volontaire à lire le premier énoncé à haute voix et demandez la réponse de chaque équipe. Clarifiez la réponse correcte et marquez un point pour l'équipe (ou les deux) dont la réponse est juste. Éclaircissez les malentendus et répondez aux questions éventuelles. Répétez pour chaque énoncé (1 minute par énoncé).

4 Après 20 minutes (17 énoncés), annoncez l'équipe gagnante. Demandez pourquoi il y a tant de mythes sur le sexe et le comportement sexuel.
Si vous prévoyez un questions-réponses de suivi :

5 Distribuez les bandelettes et expliquez:

- La plupart des gens ont des questions ou des doutes sur le sexe et le comportement sexuel. Écrivez-en une sur votre bandelette. N'écrivez pas votre nom, mais faites preuve de maturité et ne posez que des questions sérieuses. Si vous n'avez aucune question, écrivez « aucune question ».

- Pliez votre bandelette en deux.

- Je répondrai plus tard à toutes les questions sérieuses. Je vous fais confiance pour n'en soumettre aucune immature ou inappropriée (je n’y répondrai d'ailleurs pas).

6 Après quelques minutes, dites aux élèves de plier leur bandelette et ramassez-les dans une boîte ou une grande enveloppe. Quand les élèves ont quitté la classe, lisez les questions. Pour celles auxquelles vous ne pouvez répondre avec assurance, consultez une source fiable de manière à apporter une information correcte aux élèves.

Réservez un peu de temps lors d'une réunion ultérieure pour donner vos réponses aux élèves. 


\section{COMPORTEMENT SEXUEL — VRAI OU FAUX?}

\section{Lisez les énoncés et décidez s'ils sont vrais (V) ou faux (F). Inscrivez vos réponses dans la case de droite.}

V ou F ?

\begin{tabular}{|c|c|}
\hline 1 & La masturbation est nocive. \\
\hline 2 & Si une fille ne saigne pas lors de ses premiers rapports sexuels, c'est qu'elle n'est pas vierge. \\
\hline 3 & II revient à l'homme d'entamer l'activité sexuelle. \\
\hline 4 & On peut faire l'amour et avoir un orgasme sans pénétration sexuelle. \\
\hline 5 & La masturbation fréquente est un problème. \\
\hline 6 & Pour beaucoup de femmes, la pénétration vaginale seule ne suffit pas à susciter l'orgasme. \\
\hline 7 & Les premiers rapports sexuels d'une femme sont douloureux. \\
\hline 8 & La masturbation aide les gens à apprendre et comprendre la réponse de leur corps à la stimulation sexuelle. \\
\hline 9 & Une personne homosexuelle désire avoir des rapports sexuels avec n'importe quelle autre du même genre. \\
\hline 10 & Une fois qu'un homme est sexuellement excité, il ne peut plus se maîtriser et doit éjaculer. \\
\hline 11 & La meilleure façon de savoir comment faire plaisir à son ou sa partenaire est de le lui demander et d'en parler. \\
\hline 12 & Les rapports buccogénitaux peuvent transmettre une infection sexuellement transmissible (IST). \\
\hline 13 & Beaucoup d'hommes perdent leur érection lors d'un acte sexuel à un moment ou l'autre de leur vie. \\
\hline 14 & Plus le pénis d'un homme est grand, plus sa partenaire a de plaisir. \\
\hline 15 & Une femme qui aime énormément le sexe ne peut être fidèle à son partenaire. \\
\hline 16 & Le bon âge pour avoir des rapports sexuels est 18 ans. \\
\hline 17 & Si un homme peut tenir une pénétration vaginale suffisamment longtemps, la femme atteindra l'orgasme. \\
\hline
\end{tabular}




\section{réponses à « comportement sexuel — vrai ou faux ? ॥}

1 Faux La masturbation n'est pas nocive. Il s'agit plutôt d'une bonne manière, et sûre, de découvrir son propre corps. Le choix est cependant personnel. La plupart des gens se masturbent, mais certains choisissent de ne pas le faire, et l'idée met certaines personnes mal à l'aise.

2 Faux La rupture de l'hymen cause un léger saignement. Cependant, l'hymen peut facilement se détendre ou se déchirer lors d'une activité physique ou sportive ordinaire, ou lors de l'introduction de doigts ou de tampons. L'absence d'hymen ou de saignement ne veut pas dire que la fille a déjà eu des rapports sexuels.

3 Faux Dans de nombreuses cultures, il revient aux hommes d'entamer les rapports sexuels et les femmes sont censées assumer un rôle plus passif. Cette tendance reflète certaines attitudes au sujet des rôles de genre. En réalité, les femmes peuvent aussi engager l'activité sexuelle. Même celles qui se conforment aux rôles de genre traditionnels développent souvent une approche indirecte pour communiquer leur désir à leur partenaire.

4 Vrai Faire l'amour peut prendre de nombreuses formes (caresses, baisers, massage, stimulation manuelle). II est possible d'atteindre l'orgasme rien qu'en se touchant ou en se frottant l'un l'autre.

5 Faux La masturbation fréquente n'est pas un problème. Le seul moment où elle peut être considérée problématique est quand elle perturbe les autres activités requises de la personne ou que la personne en perturbe d'autres ou nuit autrement à son bien-être.

6 Vrai La plupart des femmes n'atteignent pas l'orgasme sous le seul effet de rapports par pénétration vaginale. Elles l'atteignent plutôt par stimulation du clitoris. Les femmes sont plus susceptibles d'avoir un orgasme si elles (ou leur partenaire) stimulent directement leur clitoris avant, pendant ou après le rapport vaginal.

7 Faux Les premiers rapports sexuels de la femme ne sont pas toujours douloureux. Pour minimiser la gêne ou la douleur, les partenaires doivent prendre le temps d'explorer le corps l'un de l'autre et d'être totalement excités avant la pénétration, pour que le vagin de la femme soit bien lubrifié. Si la femme est nerveuse ou anxieuse, il vaut parfois mieux attendre.

8 Vrai La masturbation est l'un des meilleurs moyens de découvrir et de comprendre la réponse de son corps à la stimulation sexuelle. Elle peut aider les femmes et les filles à apprendre à atteindre l'orgasme.

9 Faux Chaque personne, hétéro-, homo- ou bisexuelle, n'est attirée sexuellement que par certaines autres personnes. L'attirance sexuelle est le produit de nombreux facteurs.

10 Faux Certains hommes croient que s'ils sont sexuellement excités, ils doivent avoir un orgasme, mais cela est faux. L'arrêt peut causer une certaine gêne, mais qui diminue et disparaît d'elle-même. Tout le monde - homme ou femme - peut s'arrêter à tout moment d'un acte sexuel. 


\section{réponses à « comportement sexuel — vrai ou faux ? »}

11 Vrai Chaque personne a ses propres préférences en ce qui concerne l'excitation sexuelle. Plutôt que de deviner ce qui plaît ou non à son ou sa partenaire, il est plus rapide et plus fiable de le lui demander. La communication est l'une des clés d'une relation sexuelle positive source de plaisir pour les deux partenaires.

12 Vrai Les rapports buccogénitaux peuvent transmettre différentes IST : herpès, blennorragie, papillomavirus (cause de verrues ou de cancer), syphilis, chlamydia, hépatite B et chancre mou, et même le VIH.

13 Vrai Beaucoup d'hommes vivent cette expérience à un moment ou l'autre de leur vie. Il n'y a rien d'anormal à cela et il n'y a pas de quoi s'inquiéter. L'anxiété à son égard favorise la récidive.

14 Faux La taille du pénis n'a rien à voir avec le plaisir donné à la femme. Bien que les avis féminins diffèrent, la plupart disent que c'est ce que l'homme fait, et non la taille de son pénis, qui compte. En fait, un très long ou gros pénis peut être gênant ou même douloureux pour la femme.

15 Faux Il est naturel d'aimer le sexe pour les hommes comme pour les femmes. Cela n'a rien à voir avec le fait d'être digne de confiance ou non. L'idée à la base de cet énoncé, selon laquelle les femmes ne devraient pas aimer le sexe, reflète la double mesure qui admet qu'il est acceptable, et même attendu des hommes, mais pas des femmes, qu'ils aiment le sexe. L'idée est injuste, inexacte et stéréotypée.

16 Faux II n'y a pas d'âge spécifique pour avoir des rapports sexuels. Chaque personne doit déterminer elle-même le moment où elle se sent prête, suivant son niveau de maturité, sa relation, ses valeurs et ses sentiments. Certains pays ont adopté des lois sur l'âge auquel une personne est jugée capable de consentir à une activité sexuelle.

17 Faux Les rapports vaginaux ne mènent pas à l'orgasme pour de nombreuses femmes, quelle que soit la durée de la pénétration. Les femmes atteignent plus souvent l'orgasme sous l'effet de la stimulation directe du clitoris. La communication honnête entre les partenaires peut aider les deux à découvrir le plaisir sexuel. 


\section{pourquoi (ou pourquoi pas) : exercice de choix forcé sur les décisions sexuelles}

APERC̣U : Les élèves choisissent s'ils sont d'accord ou non avec une série d'énoncés sur les raisons d'avoir - ou non - des rapports sexuels.

OBJECTIFS: Aider les élèves à réfléchir de manière critique à leurs propres décisions sexuelles et à celles des autres ; renforcer la réflexion personnelle et l'expression écrite.

\section{DURÉE :}

35-40 minutes

\section{MATÉRIEL :}

Tableau noir + craie; copies

de l'exercice de choix forcés.

\section{PRÉPARATION :}

Supprimez de la liste les énoncés non appropriés et ajoutez ceux qui vous seraient utiles. D'un côté du tableau, écrivez « D'accord» et de l'autre, « Pas d'accord ».

\section{INSTRUCTIONS}

1 Présentez l'activité :

- Nous allons parler aujourd'hui de certaines des raisons pour lesquelles certains décident d'avoir des rapports sexuels et d'autres non. Beaucoup de circonstances et sentiments, parfois mixtes, influencent cette décision.

- Pour explorer le sujet, je vais lire quelques énoncés de valeur, sans réponse juste ni fausse. Si vous êtes d'accord, rendez-vous du côté «D'accord» du tableau. Si vous n'êtes pas d'accord, allez de l'autre côté. " Forcez-vous » à choisir un côté ou l'autre, même si votre opinion se trouve quelque part entre les deux. Quelquesuns d'entre vous auront ensuite l'occasion de partager leur point de vue. [Vérifiez que tout le monde comprend bien les règles.]

2 Lisez chaque énoncé et donnez le temps aux élèves de choisir leur " côté ». Invitez deux commentaires de chaque côté. Passez à l'énoncé suivant même si la conversation n'est pas terminée. Lisez autant d'énoncés que possible.
3 Réservez 5 à 10 minutes à la conclusion :

- Pourquoi faut-il que les jeunes réfléchissent clairement aux raisons de leur choix d'avoir ou non des rapports sexuels? [Sondez : sentiment d'aise, sécurité, volonté et plaisir, et protection de sa santé.]

- Les jeunes ont beaucoup de raisons de choisir ou non d'avoir des rapports sexuels. Quels types de malentendus ou de problèmes peuvent résulter de ces différences?

- Comme nous l'avons vu, on n'est pas toujours tout à fait conscient de ses motivations et de ses sentiments, ou on n'a pas toujours analysé toutes les circonstances. Comment mieux savoir ce qui se passe, ce qu'on ressent, qu'on veut ou ne veut pas? [Sondez: tenir un journal, parler à une personne de confiance, réfléchir honnêtement.

Devoir : Rédigez deux ou trois paragraphes sur un sujet de votre choix (pas nécessairement sexuel), concernant lequel vous avez des sentiments mixtes ou vous avez du mal à prendre une décision. 


\section{exercice de « choix forcés ॥}

1 Je plains les garçons car ils doivent prétendre qu'ils rêvent toujours de sexe même s'ils n'en veulent pas.

2 Si une fille aime son petit ami, elle doit le lui montrer en ayant des rapports sexuels avec lui.

3 La plupart des adolescents ont des sentiments contradictoires sur le sexe : ils en veulent et n'en veulent pas tout à la fois.

4 Je ne vois rien de mal à donner de l'argent ou un cadeau à quelqu'un en échange de rapports sexuels.

5 Je ne vois rien de mal à accepter de l'argent, si on en a besoin, en échange de rapports sexuels.

6 Un homme digne de ce nom se doit de prendre des risques et d'être sexuellement agressif.

7 Les images présentées à la télé et dans les magazines incitent les jeunes à avoir des rapports sexuels.

8 Faire pression sur quelqu'un pour avoir des rapports sexuels, contre son gré et même sans recourir à la force physique, revient plus ou moins à un viol.

9 Certaines filles donnent l'impression de ne rechercher que le plaisir sexuel, sans investissement affectif, mais au fond d'elles-mêmes, elles recherchent véritablement un lien affectif.

10 Beaucoup de filles que je connais ont des rapports sexuels parce qu'elles s'y sentent obligées.

11 Les personnes attirées par d'autres du même sexe devraient attendre plus longtemps (jusqu'à ce qu'elles soient plus âgées) avant de vivre les expériences sexuelles que leurs pairs hétérosexuels vivent déjà.

12 Beaucoup de jeunes ne veulent pas avoir de rapports sexuels. Cela n'a rien à voir avec le sida, la peur d'une grossesse ou ce que les adultes leur disent. Ils n'en veulent tout simplement pas, même s'ils ont un petit ami ou une petite amie.

13 L'acte sexuel représente toujours une expérience extrêmement intime et personnelle pour les deux participants.

14 Beaucoup de gens qui décident d'avoir des rapports sexuels le regrettent plus tard.

15 Beaucoup de gens qui décident de ne pas avoir de rapports sexuels le regrettent plus tard.

16 Avant d'avoir des rapports sexuels, la plupart des adolescents décident en parlant avec leur partenaire qu'ils sont tous deux à l'aise et qu'ils désirent ces rapports. Ils parlent aussi de leur protection contre l'infection et la grossesse. 


\section{sur la ligne : le continuum entre le choix sexuel et la contrainte}

APERC̣U : Les élèves placent des études de cas sur le continuum entre les rapports sexuels forcés et ceux volontaires et désirés et discutent le droit de refuser. (Remarque : Il importe qu'ils comprennent déjà les notions de violence sexuelle et de consentement.)

OBJECTIFS : Aider les élèves à décrire le continuum des choix sexuels ; à reconnaître les cas où les rapports sont volontaires mais non désirés; à bien comprendre le droit de refuser; renforcer la réflexion abstraite.

DURÉE :

45 minutes

\section{MATÉRIEL :}

Tableau noir + craie; copies des « études de cas de choix et contrainte sexuels »; longue corde ou ficelle solide; trombones (ou ruban adhésif); marqueurs de couleur, si possible.

\section{PRÉPARATION :}

Modifiez le document à distribuer comme indiqué (voir « études de cas de choix et contrainte sexuels »). Faites quatre copies du groupe d'études choisi.

\section{INSTRUCTIONS}

1 Fixez la corde à l'horizontale à travers la pièce. À une extrémité, marquez « Totalement forcés, non désirés » et à l'autre, « Totalement volontaires, désirés ».

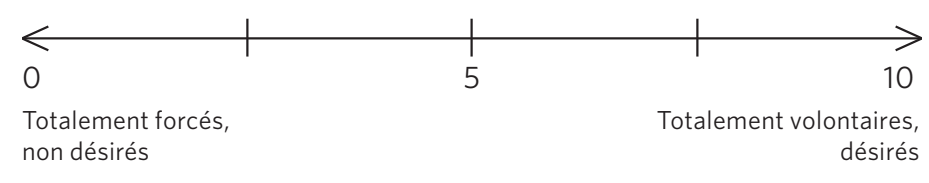

2 Présentez le sujet et expliquez qu'il existe un continuum entre les rapports sexuels forcés et absolument non désirés et ceux pleinement volontaires et désirés. Demandez :

- Quel mot utilise-t-on pour les rapports sexuels forcés? Obtenez la réponse des élèves, puis ajoutez le mot «viol » à l'extrémité du continuum.

- Si une personne accepte d'avoir des rapports sexuels, cela veut-il toujours dire qu'elle les désire vraiment? Attendez la réponse et demandez : Quand les rapports sont volontaires mais pas vraiment désirés, où se trouve-t-on sur le continuum : À l'une des extrémités ou au milieu?
3 Répartissez les élèves en quatre groupes. Donnez à chacun la feuille de cas et quelques trombones. Expliquez :

- Lisez chaque scénario avec votre groupe, parlez-en entre vous et décidez de l'endroit où il se situe dans le continuum.

- Écrivez vos noms [au marqueur de couleur si possible]. Puis attachez le cas, à l'aide d'un trombone, à l'endroit de la corde où vous avez décidé qu'il se situe.

4 Circulez parmi les groupes pour les aider. Laissez-les discuter leurs points de vue même si cela veut dire qu'ils ne couvriront pas tous les cas.

5 Au bout de 15 minutes, invitez un membre d'un groupe à lire le premier cas et à expliquer où son groupe l'a placé sur l'échelle $0-10$ et pourquoi. Donnez 2 à 3 minutes aux autres groupes pour qu'ils indiquent leur position et encouragez-les à discuter leurs différences d’opinion. Répétez pour chaque étude de cas. 
6 Réservez 10 minutes à la fin pour discuter les questions suivantes :

- Forcer quelqu'un à avoir des rapports sexuels non désirés est une violation de droits humains. Levez la main si vous pensez que les rapports forcés sont courants parmi les jeunes. Et rares?

- Nous avons vu qu'une personne qui ne désire pas de rapports sexuels et n'y est pas forcée peut toujours en avoir volontairement. Pensez-vous qu'il en aille souvent ainsi parmi les jeunes? Ou bien est-ce rare? [Remarquez si les garçons et les filles répondent différemment à cette question.]

- Les filles sont-elles aussi susceptibles que les garçons d'avoir des rapports dans des situations non forcées mais pas vraiment désirées non plus? [Sondez: Pensez-vous que les filles et les garçons ont généralement les mêmes pouvoirs dans les relations sexuelles? Et les hommes et les femmes adultes?] [Remarque: Soulignez que même si une situation ne se classe pas à l'extrémité de "rapports forcés" du spectre, elle peut toujours être inacceptable.]

- Une personne sait-elle toujours si son ou sa partenaire désire vraiment des rapports sexuels? Comment s'en assurer? [Sondez: Demandez-lui! En parler, ensemble, à l'avance, est la meilleure approche. Et si votre partenaire n'est pas sûr(e) de ce qu'il ou elle désire ?] 


\section{études de cas de choix et contrainte sexuels}

INSTRUCTIONS POUR L'ENSEIGNANT : Sélectionnez six à huit des études de cas ci-dessous (ou imaginez-en d'autres). Veillez à ce que votre sélection finale inclue au moins une situation où un garçon se sent poussé à avoir des rapports sexuels. Adaptez les cas suivant à votre contexte.

MOHAMMED et AMMA : Mohammed désire sa femme Amma mais elle n'a pas envie de rapports sexuels ce soir. Elle a pourtant appris que le devoir d'une épouse est de se plier au désir de son mari, sauf si elle est malade ou a ses règles. Elle a donc des rapports avec Mohammed.

NINA et CARL : Nina, 22 ans, sort avec Carl depuis environ six mois. Il lui a dit plusieurs fois qu'il l'a désire vraiment, mais seulement si elle le désire aussi. Nina n'est pas sûre, mais elle pense qu'elle devrait faire ce que son petit ami lui demande. Elle sait que d'autres jeunes femmes ont des rapports avec leurs petits amis et craint qu'il ne l'a quitte si elle refuse, même si Carl ne l'en a jamais menacée. Lors de leur rencontre intime suivante, ils ont des rapports sexuels.

JACOB et GRACE : Jacob et sa petite amie Grace sont seuls chez ses parents. Jacob, ivre, ne parle plus clairement et se couche par terre. Grace, qui a bu un peu trop aussi, se couche à côté de lui et, libérée de ses inhibitions, prend l'initiative et a des rapports buccogénitaux avec lui. Jacob, déconcerté, ne sait que dire. Il ne se souvient de rien le lendemain.
HENRY et MARIA : Henry et Maria s'embrassent passionnément. Quand il commence à la déshabiller, Maria essaie de l'arrêter et lui dit non. Henry pense qu'elle en veut plus mais qu'elle a peur de l'admettre. Il continue donc. Après avoir essayé de le repousser et lui avoir dit non pendant 5 minutes, elle finit par arrêter de résister et reste là, soumise. Henry a des rapports sexuels avec elle.

AJIT et ANILA : Ajit et Anila ne se sont vus qu'une ou deux fois, toujours sous surveillance. Ajit lui semble gentil et Anila accepte quand ses parents lui disent qu'il désire l'épouser. Anila a appris que tout ce qui est sexuel est honteux. Elle a entendu dire que les premiers rapports sexuels font mal et la feront saigner. Elle a vraiment peur. Elle connaît à peine Ajit et se sent honteuse à l'idée qu'il touche son corps. Elle n'a pas envie de rapports sexuels et ne se sent pas excitée, mais elle sait que quand on se marie, on doit avoir des rapports la nuit de noces. Elle laisse Ajit avoir des rapports avec elle. 


\section{études de cas de choix et contrainte sexuels}

SELAH et JAMES : La dernière fois que Selah a refusé son mari James, il l'a menacée et battue au point de lui donner un œil au beurre noir. Elle ne veut pas avoir de rapports sexuels avec James aujourd'hui mais, de peur qu'il la batte encore, elle ne refuse pas.

LIN et YANG : Le petit ami de Lin, Yang, fait vraiment pression pour qu'elle accepte d'avoir des rapports sexuels avec lui. Elle n'en veut pas vraiment, mais elle a accompagné Yang dans un endroit isolé et accepte sans mot dire «l'inévitable ».

TEO et HELENA : Teo et Helena se sont rencontrés à l'université. Ils sortent ensemble depuis quelques mois et sont très attirés l'un envers l'autre. Ils ne sont pas profondément amoureux mais décident qu'ils veulent faire l'amour ensemble. Ils acceptent d'utiliser le préservatif et ont des rapports sexuels.

GINA et AHMED : Gina vient d'une famille pauvre. Elle travaille pour Ahmed et son salaire est le revenu principal de sa famille. Un jour, Ahmed se met à la toucher et, après l'heure de fermeture, l'attire dans l'arrière-boutique et soulève sa robe. Déconcertée, Gina a peur de perdre son emploi. Elle tente de résister mais, quand Ahmed lui dit : "Quoi ? Tu n'aimes pas travailler ici ? ", elle cède.
JULIA et OSCAR : Oscar n'est pas sûr de vouloir avoir des rapports sexuels avec Julia, mais elle les a suggérés et il craint que ses amis ne l'apprennent et ne le taquinent s'il refuse. Il accepte donc.

PETER et LAURA : Peter achète souvent des cadeaux et d'autres nécessités à Laura. Ce soir, ils sont allés au restaurant et il a payé. Laura ne se sent pas prête à avoir des rapports sexuels mais elle pense les devoir à Peter et ne le refuse donc pas.

VERA et WILLIAM : Vera et William ont déjà fait l'amour ensemble une fois. Ce soir, William lui dit qu'il l'a désirée toute la journée. Vera préférerait simplement être ensemble et discuter, mais elle pense que comme ils l'ont déjà fait une fois, il n'y a plus d'autre issue. Quand William se déshabille, elle ne dit rien et ils ont de nouveau des rapports.

EMMA et ROBERT : Un soir, Emma a trop bu. Robert la trouve allongée sur le lit, ivre morte. Il la déshabille et a des rapports sexuels avec elle. Elle ne se réveille pas. 


\section{question de consentement}

APERC̣U : Les élèves apprennent le concept du consentement sexuel. Ils l'explorent à travers l'étude de cas, la mise en scène et la discussion.

OBJECTIFS : Aider les élèves à comprendre et apprécier l'importance du consentement dans toutes les situations sexuelles; renforcer la réflexion abstraite.

\section{DURÉE :}

Étapes 1-6:45 minutes

Étapes $7-8: 45$ minutes

\section{MATÉRIEL :}

Tableau noir + craie ; « études de cas sélectionnées »;

« Info enseignant »; «Clé

pour l'enseignant ».

\section{PRÉPARATION :}

Sélectionnez quatre à six études de cas reflétant des situations courantes dans votre contexte et adaptez-les en changeant les noms ou d'autres détails. Ou bien élaborez vos propres cas. Choisissez quelques cas où les personnages peuvent donner leur consentement librement et en connaissance de cause et d'autres où ils ne le peuvent pas. Au besoin référez vous à « Info enseignant ». Écrivez les directives au tableau avant la classe. Renseignez-vous sur l'âge légal du consentement à l'activité sexuelle dans votre pays.

\section{INSTRUCTIONS}

1 Présentez l'activité au moyen de l'explication et des questions suivantes :

- Nous avons tous des obligations : à la maison, à l'école, au travail. Il arrive cependant que nos pairs ou même des adultes nous demandent ou nous disent de faire quelque chose qui n'entre pas dans nos obligations, et qui peut être déconcertant ou troublant.

Option: Pour présenter le concept en détail, étendez la discussion comme suit :

Que veut dire l'idée de " consentir » à quelque chose? [Sondez: être d'accord, indiquer ou exprimer une volonté spécifique.] On consent à quelque chose pour différentes raisons : parce qu'on en a envie, si un ami nous invite par exemple à jouer au football. On peut aussi consentir à quelque chose dont on n'a pas vraiment envie parce que c'est important pour d'autres raisons : quand un prof nous donne un devoir qui nous déplaît, par exemple, mais qu'on le fait quand même parce qu'on veut réussir à l'école.
Mais il arrive qu'on fasse quelque chose qu'on ne désire pas simplement parce qu'il est difficile de se défendre ou qu'on ne veut pas être impoli. Le problème peut être sans conséquence, quand on mange quelque chose qu'on n'aime pas pour faire plaisir à la personne qui l'a préparé. Mais il peut aussi s'agir parfois de choses graves, et lourdes de conséquences. Donnez-moi des exemples de choses graves auxquelles un jeune pourrait consentir ou non? [Si le sexe n'est pas mentionné, suggérez de l'ajouter à la liste.]

- Levez la main si vous avez jamais accepté de faire quelque chose qui ne relevait pas de vos obligations et que vous ne vouliez pas vraiment faire. Pouvez-vous partager un exemple?

- Pourquoi accepte-t-on parfois de faire quelque chose dont on n'a pas vraiment envie? [Écrivez les réponses données au tableau.]

- Nous allons aujourd'hui commencer à considérer ce que c'est que de consentir librement et en connaissance de cause à une situation de nature sexuelle. 
2 Demandez (et écrivez les réponses au tableau) :

- Que veut dire le mot « consentement» ?

- Qu'est-ce qu'un « consentement libre et en connaissance de cause » ?

3 Invitez les élèves à lire à haute voix les règles d'un consentement libre et en connaissance de cause dans une situation de nature sexuelle.

4 Formez de petits groupes et remettez à chacun une étude de cas différente. Expliquez:

- Vous avez 10 minutes pour discuter votre cas, préparer un petit sketch (2 à 3 minutes) et considérer les règles de consentement qui y sont remplies ou non.

- Une personne va lire le cas à la classe au complet, deux vont le jouer et une ou deux autres vont expliquer les règles de consentement remplies ou non. [Prévoyez 10 à 20 minutes pour clarifier la tâche et laisser les groupes se préparer. Circulez parmi eux pour les aider.]

5 Invitez un groupe à présenter a) son cas ; b) son sketch ; et c) son opinion collective du consentement libre et en connaissance de cause ou non de la personne représentée.

6 Les autres élèves sont-ils d'accord ou non avec l'opinion du groupe concernant l'observation des règles de consentement? Discutez jusqu'à obtenir le consensus sur la réponse correcte.
7 Répétez les étapes 5 et 6 pour chaque groupe/cas. Prévoyez 10-12 minutes par cas.

8 Pour conclure, animez la discussion : nous savons qu'il existe des circonstances où une personne n'a pas la possibilité de dire non. Il s'agit d'une violation de ses droits humains.

- Souvent, le degré de contrôle ou de pouvoir d'une personne n'est pas clair ou peut être ouvert à la négociation. Il suffit parfois simplement de comprendre, au fond de soi, qu'on a le droit de consentir ou non à des rapports sexuels. Il s'agit là $\mathrm{du}$ « pouvoir de la connaissance ».

- Une personne peut-elle être certaine du consentement de son ou sa partenaire sans lui parler? Pourquoi ou pourquoi pas? Comment s'assurer qu'une personne « consentante » à des rapports sexuels l'est librement et en connaissance de cause?

Devoir : Mettez-vous à la place d'une personne qui s'est sentie obligée d'avoir des rapports non désirés. Pensez au personnage d'une étude de cas lue en classe aujourd'hui ou à quelqu'un que vous connaissez qui s'est trouvé(e) dans une situation de consentement inadéquat (n'utilisez pas son vrai nom). Écrivez un discours en son nom. Mettez l'accent, non pas sur ce qui s'est passé précisément, mais sur ses sentiments. Pensez aux différences de pouvoir qui sont peut-être entrées en jeu dans le sentiment de pression. Votre but est d'aider la personne à « reconquérir » son sens du contrôle et de l'égalité de pouvoir. Si vous le préférez, écrivez votre allocution sous forme de poème. 


\section{règles de consentement véritable dans une situation de nature sexuelle}

Croire qu'on a le droit de décider soi-même si l'on veut ou non participer à une activité sexuelle particulière.

Avoir un sentiment suffisant de pouvoir et de maîtrise de sa propre vie pour pouvoir communiquer et exécuter sa décision.

Avoir la maturité de comprendre ce qu'implique l’activité envisagée et les conséquences dont elle peut s'accompagner, et la maturité de dialoguer avec son partenaire sur la question.

Être dans une situation ou une relation où sa décision sera reconnue et respectée par le ou la partenaire.

Savoir ce qu'implique l'activité et les sentiments qu'on ressent à son égard, les risques qu'elle représente et comment se protéger, soi-même et son ou sa partenaire, d'une grossesse non désirée ou d’une infection.

Avoir l'esprit clair, non influencé par l'alcool ou la drogue au moment de la décision.

Être correctement informé(e) de l'état de santé sexuelle actuel de son ou de sa partenaire (y compris toute exposition sexuelle ou usage de drogues antérieurs susceptibles de présenter un risque).

Autant que possible, éviter les situations propices aux pressions sexuelles pour raisons matérielles ou financières. 


\section{études de cas : cette personne peut-elle vraiment consentir?}

A Ana a 19 ans et son petit ami, Al, 22 ans. Ils sortent ensemble depuis six mois et ont des rapports sexuels depuis environ un mois. Ana y prend généralement plaisir, mais elle n’en a pas toujours envie. Al lui dit alors souvent que si elle l'aimait vraiment, elle en aurait envie, ou si elle le rejette, peut-elle devra-t-il trouver quelqu'un d'autre. Ana ne veut pas le perdre ; elle cède donc souvent. Peut-elle consentir librement?

B Beni se sait séropositif à VIH mais n'en a rien dit à personne. Il sort avec Bea et ils parlent depuis peu d'avoir des rapports sexuels. Beni entend utiliser le préservatif plutôt que de révéler son état à Bea. Bea peut-elle consentir librement et en connaissance de cause?

C Carlo et Clea ont entre 20 et 30 ans. Ils sortent ensemble depuis un an et se sont parlés franchement de leurs relations passées. Ils aimeraient avoir des rapports sexuels ensemble. Ils en ont parlé et ont décidé d'aller dans une clinique et de décider ensemble comment avoir des rapports sans risques et sans conséquences non désirées. Clea peut-elle consentir librement et en connaissance de cause?

D Dom et Deenah ont 18 ans et ont décidé d'avoir des rapports sexuels. Deenah a peur de se retrouver enceinte. Dom lui assure qu'on ne peut pas tomber enceinte la première fois. Deenah peut-elle consentir librement et en connaissance de cause?

E Eve a 14 ans et est lycéenne. Edo a 18 ans et travaille avec le père d'Eve. Edo a rencontré Eve lors d'une visite à son père. Edo et Eve ont commencé à se voir à l'extérieur. Edo offre parfois des cadeaux à Eve, et de l'argent quand elle en a besoin. Depuis peu, il lui dit combien il l'aime et qu'il la désire vraiment. Eve peut-elle consentir librement et en connaissance de cause?

F Finn a 18 ans et voudrait avoir des rapports sexuels avec sa petite amie Fatima, qui a 17 ans. Ils ont parlé du fait que ni l'un ni l'autre n'a encore eu de rapports sexuels. Il décide de lire un livre que sa mère, médecin, a sur le fait de grandir, la planification familiale et les IST. Il demande à Fatima ce qu'elle pense et lui prête le livre. Elle le lit et ils en reparlent ensemble. Elle dit qu'elle préférerait attendre et il accepte. Fatima peut-elle consentir librement et en connaissance de cause? 


\section{études de cas : cette personne peut-elle vraiment consentir?}

G Gia (24 ans) boit beaucoup lors une fête chez des amis étudiants. Son partenaire, avec qui elle a déjà eu des rapports sexuels, l'emmène dans une chambre et commence à la déshabiller. Elle est presque ivre morte. Gia peutelle consentir librement et en connaissance de cause?

H La mère de Halle lui a appris qu'il est important pour une femme de faire tout ce que son mari demande d'elle si elle ne veut pas le perdre. Elle est fiancée à Hugh, qui lui dit que maintenant qu'ils vont se marier, ils peuvent avoir des rapports sexuels. Halle croit profondément qu'elle devrait attendre et veut rester vierge jusqu'à son mariage. Elle pense cependant : «Il va être mon mari et ma mère dit que je dois lui obéir. » Halle peut-elle consentir librement et en connaissance de cause?

I Isaac a eu plusieurs partenaires mais laisse Ivan, son nouvel ami, croire qu'il est toujours vierge, comme lui. Ivan accepte d'avoir des rapports sexuels sans préservatif, pensant qu'il ne court aucun risque d'infection. Ivan peut-il consentir librement et en connaissance de cause?

J Jin a 20 ans et Josef en a 19. Il y a environ six mois, Jin a demandé à Josef, son petit ami, d'avoir des rapports sexuels avec elle. Ils sont ouverts et honnêtes l'un envers l'autre et il lui a donc dit qu'il avait peur des conséquences et qu'il ne se sentait pas prêt. Il préférerait juste qu'ils s'embrassent et se touchent pour le moment. Jin a accepté. Josef pense maintenant être prêt. Il demande à Jin où ils peuvent se renseigner pour être sûr d'éviter tous risques. Josef peut-il consentir librement et en connaissance de cause? 


\section{réponses aux « études de cas »}

Remarque : Veillez à vous renseigner sur l'âge minimum légal requis dans votre pays pour consentir à l'activité sexuelle et déterminer si les personnages présentés dans chaque scénario ont l'âge de consentir. Au besoin, changez les scénarios ou les réponses en fonction de cette information.

A Non Al ne respecte pas la décision d'Ana quand elle lui dit qu'elle ne désire pas avoir de rapports sexuels. Elle ne croit pas non plus fermement qu'elle a le droit de décider pour elle-même et n'a pas un sentiment suffisant de pouvoir au sein de la relation pour s'en tenir à son choix.

B Non Bea ne dispose pas d'une information exacte sur l'état de santé sexuelle de son partenaire.

C Oui Ils ont décidé individuellement et sont d'accord; ils prennent leur décision l'esprit clair, sans motivation matérielle ou financière ; ils sont capables de communiquer leur décision, savent ce qui les attend et savent qu'ils ont besoin d'une protection; ils ont parlé honnêtement de leur comportement passé et ils sont légalement en âge de consentir à des rapports sexuels.

D Non Deenah ne sait ni les risques qu'elle court, ni comment se protéger.

E Non Le consentement sexuel d'une personne de 14 ans est rarement véritable, car elle n'a pas encore atteint sa pleine maturité. Les préoccupations de maturité et de droits des enfants ont donné lieu à l'adoption de lois régissant l'âge minimum requis pour pouvoir consentir à un acte sexuel et la proscription des mariages d'enfants.

F Oui Fatima sait qu'elle a le droit de décider pour elle-même et son partenaire respecte sa décision. Elle prend cette décision l'esprit clair, n'est pas influencée par une motivation économique ou matérielle, peut communiquer et exécuter sa décision, sait à quoi elle s'engage, connaît les risques et sait comment se protéger. Elle dispose d'une information correcte sur le comportement sexuel antérieur de son partenaire. Dans la plupart des pays, elle serait considérée en âge de prendre la décision d'avoir des rapports sexuels si tel est son choix mais vérifiez vos lois locales.

G Non Gia est ivre et pratiquement inconsciente. Elle ne peut pas décider pour elle-même et son partenaire ne communique pas avec elle.

H Non Halle sait ce qu'elle désire, mais elle ne semble pas croire en son droit de décider pour elle-même, ou n'a pas un sentiment suffisant de pouvoir et de maîtrise de sa propre vie pour s'en tenir à sa décision.

I Non Ivan ne dispose pas d'une information exacte sur le comportement sexuel antérieur de son partenaire.

J Oui Josef croit qu'il a le droit de décider pour lui-même ; il est capable de communiquer et d'exécuter sa décision et sa partenaire la respecte ; il a l'esprit clair, n'a pas de motivation économique ou matérielle, est conscient des risques et de son besoin de protection et entend s'informer davantage à ce sujet, et est en âge de prendre cette décision. Bien qu'on ignore si les partenaires ont parlé de leur comportement sexuel antérieur ou de leur usage de drogue passé, ils sont ouverts et honnêtes l'un envers l'autre. 


\section{actualité sexuelle et réponses personnelles}

APERCQU : Les élèves sélectionnent un titre d'actualité qui reflète les normes changeantes de la sexualité et rédigent une réponse personnelle.

OBJECTIFS : Aider les élèves à comprendre l'évolution des normes sexuelles au fil du temps et à clarifier leurs propres valeurs; renforcer l'esprit critique et l'expression écrite.

\section{DURÉE :}

45 minutes

\section{MATÉRIEL :}

Tableau noir + craie; «Titres»

d'actualité de politique sexuelle.

\section{PRÉPARATION :}

Supprimez les titres non adaptés à votre contexte. Copiez ceux qui restent au tableau.

\section{INSTRUCTIONS}

1 Présentez l'activité :

- Les attitudes sur la sexualité diffèrent d'une société à l'autre. Elles évoluent aussi au sein même de chaque société. Nous allons identifier quelques changements dans les attitudes et y réfléchir.

- Lisez la liste de titres inscrits au tableau. La plupart sont adaptés d'événements réels survenus de par le monde. Sélectionnez-en un et rédigez-y une réponse personnelle d'environ une page. Parlez de vos réactions ou de vos sentiments, en y réfléchissant profondément. En conclusion, indiquez votre opinion d'où en seront les choses sur cette question dans 10 ou 15 ans. Vous avez 5 minutes pour choisir votre sujet et 15 pour rédiger votre réponse.

2 Pour chaque titre, invitez un volontaire à lire sa réponse écrite. En fin de lecture, remerciez l'élève. Au besoin, clarifiez le problème et rectifiez toute désinformation flagrante. Encouragez la discussion :

- Que pensez-vous de cette réponse?

- Quelles seraient vos réponses?

- Ce sujet concerne-t-il votre pays?
- Avez-vous d'autres commentaires à émettre?

[Prévoyez 2 ou 3 minutes par titre. Si certains n'ont pas été sélectionnés, consacrez plus de temps à ceux qui l'ont été.]

3 Pour conclure, rappelez aux élèves que les normes sont en évolution permanente. Demandez :

- Le changement va-t-il toujours dans le sens de normes plus souples, ou peut-il y avoir un mouvement vers des normes plus strictes et restrictives?

- Les normes sociétales — au sujet de la sexualité ou à d'autres égards - changent-elles d'ellesmêmes, comme par magie? Qui ou quoi fait changer les normes sexuelles? [Sondez: larges phénomènes sociaux (éducation supérieure et médias mondialisés) et rôles individuels (leaders publics et membres de la communauté).]

- Des hommes et des femmes sont à l'origine de ce changement : ils se font modèles à suivre, ils sensibilisent l'opinion et ils militent pour le changement des politiques. 


\section{« titres » d'actualité de politique sexuelle}

Au tribunal, première victoire féminine contre le harcèlement sexuel

Ouverture d'une clinique pour adolescents : la première de l'État

Manifestation masculine contre la violence sexuelle: les hommes dignes de ce nom ne violent pas

La loi autorisant les mariages homosexuels entre en vigueur : premier couple marié à 8 heures ce matin

Jeune fille de 14 ans échappe au mariage forcé

Les filles protestent : non au port permanent de la burka

60 jeunes hommes signent une promesse de n'épouser que des filles non excisées

Arrestation d'un homme séropositif qui cherchait à se "guérir " par des rapports sexuels avec cinq vierges

Nouvelle étude : les parents continuent à vendre leurs filles aux trafiquants du sexe

Le Président: "Mon attitude envers l'homosexualité a changé quand j'ai appris que ma fille était lesbienne.»

Renforcement de la loi contre l'IVG — prestataires arrêtés 


\section{mes relations}

activité 27

\section{DURÉE :}

45 minutes

\section{MATÉRIEL :}

Tableau noir + craie

\section{PRÉPARATION :}

Aucune, sinon la lecture des instructions.

\section{APERCQU : Les élèves créent une représentation visuelle de leurs relations.}

OBJECTIFS : Aider les élèves à identifier leurs différentes relations et à nommer les qualités qu'ils apprécient en eux-mêmes et qu'ils recherchent dans leurs relations proches ; renforcer la réflexion critique.

\section{INSTRUCTIONS}

1 Expliquez aux élèves que vous allez identifier leurs différents types de relations. Demandez-leur de dresser la liste de leurs relations et liens personnels avec d'autres personnes.

2 Invitez ceux qui le désirent à partager leur liste. Inscrivez au tableau les différents types de relations mentionnés : parents, amis ou voisins, par exemple.

3 Tracez au tableau un diagramme composé de quatre cercles concentriques (comme illustré ici).

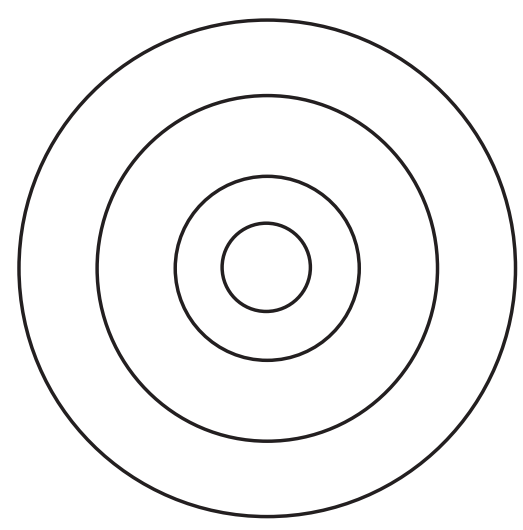

4 Demandez aux élèves de prendre une feuille de papier vierge. Expliquez :

- Tracez quatre cercles, petit à grand, comme je l'ai fait au tableau. Utilisez toute la surface de la feuille pour le plus grand.

- Dans le plus petit cercle, écrivez votre nom (ou dessinez votre portrait).

- Réfléchissez aux personnes présentes dans votre vie. Écrivez dans le deuxième cercle le nom de celles qui vous sont les plus proches (ou dessinezles).

- Dans les deux cercles extérieurs, écrivez le nom de celles plus éloignées (ou dessinez-les).

5 Invitez les élèves à former des groupes de trois ou quatre avec ceux assis près d'eux et donnez-leur 2 minutes pour expliquer leur diagramme au groupe ainsi formé. 
6 Animez la discussion en posant ces questions :

- Chaque membre de votre groupe a-t-il listé les mêmes types de relations? Chacun a-t-il placé les membres de sa famille, ses amis, voisins, enseignants, chefs religieux ou d'autres, dans les mêmes cercles ou dans des cercles différents?

- Citez quelques mots qui décrivent les qualités que vous appréciez dans les personnes dont vous vous sentez le plus proche. [Sondez: honnête, respectueux, généreux, aimant, confiant, gai, sûr, compréhensif, fiable, intéressant. Écrivez ces mots au tableau.]
7 Réservez 10 minutes à l'exploration des qualités que les élèves valorisent dans leurs relations :

- Y a-t-il sur votre diagramme une personne que vous aimeriez rapprocher, plus près de votre cercle intime? Choisissez un mot qui décrit ce que vous valorisez dans cette personne.

- Réfléchissez maintenant à un ou deux mots, parmi ceux écrits au tableau, qui vous semblent s'appliquer à vous.

- Célébrez une qualité que vous valorisez grandement (en vous-mêmes ou chez d'autres) en l'écrivant sur votre feuille en lettres ornées ou dans un style créatif.

- Tandis que vous écrivez ou dessinez ce mot, pensez à ce qu'il veut dire pour vous. 


\section{que se passe-t-il entre ces deux-là ?}

\section{8}

\section{DURÉE :}

45 minutes

\section{MATÉRIEL :}

Tableau noir + craie ; une « étude de cas » par petit groupe.

\section{PRÉPARATION :}

Lisez les études de cas proposées et adaptez ou remplacez-les de manière à assurer qu'elles reflètent des formes courantes d'inégalité dans votre société. Photocopiez chaque étude sur une feuille de papier différente.
APERC̣U : Les élèves lisent les récits de relations caractérisées par l'inégalité sociétale. Ils identifient les inégalités et discutent leur effet sur les relations.

OBJECTIFS : Aider les élèves à identifier l'effet de l'inégalité sociétale sur les relations intimes ; renforcer la réflexion analytique.

\section{INSTRUCTIONS}

\section{Expliquez:}

- Nous allons explorer aujourd'hui la manière dont l'inégalité et les différences de pouvoir peuvent affecter nos relations. Quelles sont les formes d'inégalité présentes dans notre société entre différents groupes? [Sondez: le racisme, les castes et l'inégalité basée sur des variables telles que le genre, l'âge, la classe, l'ethnie et la capacité physique.]

- Vous allez former quatre groupes, dont chacun lira une étude de cas, suivie de quelques questions de discussion auxquelles vous répondrez ensuite.

2 Donnez à chaque groupe une étude de cas et les questions de discussion. Laissez les groupes travailler pendant 10 minutes.

3 Invitez un groupe à lire son étude et à présenter ses réponses aux questions, en l'espace de 5 à 7 minutes. Prévoyez ensuite quelques minutes pour permettre aux autres d'exprimer leurs vues.
4 Procédez de même pour les trois autres études de cas.

5 Pour conclure, encouragez les élèves à identifier une inégalité, dans la société, qui affecte leurs propres relations personnelles et à réfléchir aux conséquences qu'ils en ressentent.

Devoir : Écrivez une carte postale à une personne décrite dans votre étude de cas. Donnez-lui conseil sur la manière de réduire l'impact de l'inégalité dans sa relation. 


\section{GROUPE 1 :}

J'ai été attirée par mon petit ami parce qu'il était plus âgé et que personne ne le connaissait. Il me paraissait mystérieux et excitant. Nous sommes tombés amoureux et maintenant, nous passons presque tout notre temps ensemble. Vous savez comment c'est. J'aime l'écouter raconter tout ce qu'il a déjà fait. Quand nous sortons, il décide généralement où parce que c'est lui qui paie et il connaît tous ces endroits fabuleux. Il m’a beaucoup influencée. Je n'aimais pas vraiment le sport, mais il adore le football. Je passe maintenant beaucoup de temps avec lui au bord d'un terrain de foot ou à regarder les sports à la télé.

Au bout de quelques mois, il m'a dit qu'il voulait me faire l'amour. J'ai hésité, mais j’ai eu peur qu'il me quitte si je refusais. Après tout, il a 23 ans et il a de l'expérience. Ces jours-ci, je ne vois presque plus mes amies. Elles se plaignent et disent que j’ai changé. Elles me manquent et je pense parfois aussi à combien j'étudiais avant, et combien j’aimais lire. Mais j'imagine qu'ainsi va la vie quand on est amoureuse.

\section{Questions:}

1 Il existe différents types d'inégalité entre les groupes de la société (certaines personnes occupent par exemple un rang supérieur ou inférieur suivant leur groupe ethnique ou racial, leur classe économique, leur sexe, leur âge ou d'autres caractéristiques). Quelles sont les formes d'inégalité présentes dans cette relation?

2 Quel membre de cette relation a la position de puissance en raison de son rang dans la société ? Qu'y a-t-il à la base de cette inégalité ? Comment cette position dominante est-elle démontrée?

3 Quel membre de cette relation occupe la position inférieure en raison de son rang social ? Comment cette position inférieure est-elle démontrée?

4 Quel est l'effet, s'il en est, de cette inégalité sur chaque personne ? Comment cela affecte-t-il la relation ?

5 Pensez-vous que ce genre de problèmes se pose dans notre communauté ? Comment ? 


\section{GROUPE 2 :}

L’an dernier, j'ai commencé à sortir avec un gars de l'équipe de rugby de notre village. Les choses sont devenues sérieuses entre nous. Un soir, à une réunion au centre du village, il m’a fait signe de le rejoindre derrière la salle. Ma mère et mon père étaient là, mais je savais qu'ils penseraient que j'étais avec d'autres filles. Nous sommes donc allés, lui et moi, nous promener dans la broussaille derrière la salle. Nous nous sommes embrassés et il a commencé à me toucher les seins. Je savais que nous allions avoir des rapports sexuels. Je n'en avais pas vraiment envie. Je voulais simplement lui parler, l'embrasser et rien d'autre, mais lui en voulait et je l'avais accompagné. Nous avons donc fini par en avoir.

J'ai ensuite pleuré car je savais que j'avais manqué de respect à mes parents et que j'avais perdu ma virginité. J'avais entendu parler du préservatif et je savais qu'il pouvait empêcher une fille de tomber enceinte ou d'attraper une maladie, mais j'avais d'autres choses en tête, comme ce que mes parents penseraient s'ils savaient ce que je faisais. J'étais plus inquiète à l'idée de perdre ma virginité. Quand je me suis retrouvée enceinte, mes parents ont été furieux et m'ont interdit de revoir mon petit ami.

\section{Questions :}

1 II existe différents types d'inégalité entre les groupes de la société (certaines personnes occupent par exemple un rang supérieur ou inférieur suivant leur groupe ethnique ou racial, leur classe économique, leur sexe, leur âge ou d'autres caractéristiques). Quelles sont les formes d'inégalité présentes dans cette relation?

2 Quel membre de cette relation a la position de puissance en raison de son rang dans la société ? Qu'y a-t-il à la base de cette inégalité ? Comment cette position dominante est-elle démontrée?

3 Quel membre de cette relation occupe la position inférieure en raison de son rang social ? Comment cette position inférieure est-elle démontrée?

4 Quel est l'effet, s'il en est, de cette inégalité sur chaque personne ? Comment cela affecte-t-il la relation?

5 Pensez-vous que ce genre de problèmes se pose dans notre communauté ? Comment? 


\section{GROUPE 3 :}

À 35 ans, je me suis dit qu'il était temps que je me marie. J'avais enfin un emploi et je pouvais subvenir aux besoins d'une famille. J'étais diplômé de l'université, mais je n'ai pas pu trouver de travail près de chez moi tant le chômage était élevé. J'ai fini par migrer pour trouver un emploi. Lors de mes visites chez ma mère, j’ai décidé de me mettre à chercher une épouse dans notre village. J'ai rencontré plusieurs jeunes femmes, dont une m’a beaucoup plu. Elle avait 20 ans et était de tempérament paisible. Elle avait terminé ses études primaires, mais n’avait jamais vraiment quitté le village. Bien que je lui aie dit qu'elle devrait vivre avec ma mère en attendant que je puisse l'emmener avec moi, elle a accepté de m'épouser.

Après la naissance de notre premier enfant, je ne voulais pas qu'elle prenne de contraceptifs et j'ai donc pratiqué le retrait. Je fais de mon mieux pour subvenir à ses besoins et à ceux de notre enfant. Elle vit toujours avec ma mère mais ne cause jamais de problèmes et ne dit jamais avoir besoin de quoi que ce soit. Je l'aime mais il y a des choses dont je ne peux lui parler. Elle ne comprendrait pas. Elle ne sait vraiment pas grand-chose du monde.

\section{Questions:}

1 Il existe différents types d'inégalité entre les groupes de la société (certaines personnes occupent par exemple un rang supérieur ou inférieur suivant leur groupe ethnique ou racial, leur classe économique, leur sexe, leur âge ou d'autres caractéristiques). Quelles sont les formes d'inégalité présentes dans cette relation?

2 Quel membre de cette relation a la position de puissance en raison de son rang dans la société ? Qu'y a-t-il à la base de cette inégalité ? Comment cette position dominante est-elle démontrée ?

3 Quel membre de cette relation occupe la position inférieure en raison de son rang social ? Comment cette position inférieure est-elle démontrée?

4 Quel est l'effet, s'il en est, de cette inégalité sur chaque personne ? Comment cela affecte-t-il la relation?

5 Pensez-vous que ce genre de problèmes se pose dans notre communauté ? Comment? 


\section{GROUPE 4 :}

Je suis allée travailler à l'étranger pendant quelque temps et je suis tombée amoureuse d'un collègue. Quand je me suis retrouvée enceinte, nous avons décidé de nous marier. Mes parents y étaient opposés parce qu'il venait d'une famille pauvre: «Tu ne sais pas les implications d'un mariage avec quelqu'un qui n’est pas ton égal. Pourquoi ne reviens-tu pas? Tu auras l'enfant ici. Nous t'aiderons. » Nous nous sommes mariés quand même et nous avons décidé de rentrer dans mon pays pour qu'il puisse aller à l'université pendant que je travaillais. Mais il fallait avant tout qu'il apprenne la langue.

Il a malheureusement trouvé la situation difficile. Il restait à la maison avec le bébé et essayait d'apprendre, mais il se sentait fort isolé. Il était de plus en plus déprimé et son estime personnelle était au plus bas. Il dépendait financièrement de moi et se sentait coupable car nous devions envoyer régulièrement de l'argent à sa famille. Je n'appréciais pas le fait qu'il n'étudiait pas, ne cherchait pas d'emploi et ne travaillait pas, mais j'étais gênée à l'idée d'en parler. C'était un sujet tellement sensible et je ne voulais pas le blesser. Il a fini par se lier d'amitié avec quelques hommes de son pays et s'est mis à fréquenter les bars et à boire avec eux. Il lui est même arrivé de se montrer violent envers moi. Ç’a été un véritable avertissement pour nous.

\section{Questions:}

1 Il existe différents types d'inégalité entre les groupes de la société (certaines personnes occupent par exemple un rang supérieur ou inférieur suivant leur groupe ethnique ou racial, leur classe économique, leur sexe, leur âge ou d'autres caractéristiques). Quelles sont les formes d'inégalité présentes dans cette relation?

2 Quel membre de cette relation a la position de puissance en raison de son rang dans la société ? Qu'y a-t-il à la base de cette inégalité ? Comment cette position dominante est-elle démontrée?

3 Quel membre de cette relation occupe la position inférieure en raison de son rang social ? Comment cette position inférieure est-elle démontrée?

4 Quel est l'effet, s'il en est, de cette inégalité sur chaque personne ? Comment cela affecte-t-il la relation ?

5 Pensez-vous que ce genre de problèmes se pose dans notre communauté ? Comment? 


\section{c'est quoi tomber amoureux ? l'amour vrai ? la jalousie?}

APERC̣U : Les élèves distinguent l'amour d'autres émotions.

OBJECTIFS : Aider les élèves à réfléchir aux sentiments d'amour, d'engouement et de jalousie ; renforcer la réflexion critique.

\section{INSTRUCTIONS}

1 Répartissez les élèves en cinq groupes. Invitez chacun à choisir un des petits papiers que vous avez préparés. Au tableau, écrivez : "Quelle est la différence entre l'amour et ?"

2 Demandez aux groupes de prendre une feuille de papier et de copier la phrase écrite au tableau, en la complétant du mot qu'ils ont choisi. Invitez-les à discuter leur question et à rédiger leur réponse.

3 Au bout d'environ 5 minutes, invitez un groupe à lire sa question et présenter sa réponse. Les questions suivantes peuvent guider une brève discussion :

- Êtes-vous d'accord ou aimeriez-vous ajouter quelque chose?

- La culture donne-t-elle aux filles et aux garçons la même permission de ressentir ce sentiment ?

- Pouvez-vous donner un exemple d'une situation où une personne confond ce sentiment avec de l'amour? Que se passe-t-il alors?

- Ce sentiment fait-il partie de l'amour?
4 Répétez l'étape 3 pour les quatre groupes restants

5 Réservez 10 minutes à la discussion des questions suivantes :

- Où les jeunes développent-ils leurs impressions de l'amour et de l'idylle?

- Les films et les romans sentimentaux présententils une image réaliste de ces sentiments? Si non, quel effet pensez-vous qu'ils ont sur les attentes des jeunes?

- Pourquoi est-il important que vous déterminiez pour vous-même la différence entre l'amour et ces autres sentiments?

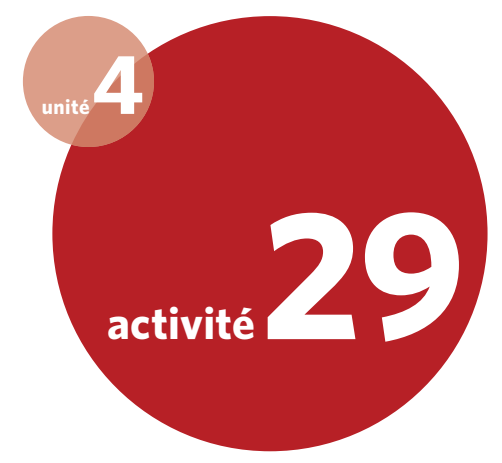

\section{DURÉE :}

45 minutes

\section{MATÉRIEL :}

Tableau noir + craie ; morceaux de papier sur lesquels vous avez écrit les mots ci-dessous.

\section{PRÉPARATION :}

Modifiez la leçon selon les besoins de votre culture, du contexte et de la langue des élèves. Prenez cinq morceaux de papier et écrivez sur chacun l'un des mots ou expressions suivants :

- L'ENGOUEMENT

- ÊTRE «AMOUREUX»

- L'IDYLLE

- L'ATTIRANCE SEXUELLE

- LA JALOUSIE 


\section{décider d'avoir ou non des rapports sexuels}

\section{DURÉE :}

45 minutes

\section{MATÉRIEL :}

Tableau noir + craie; copies de la feuille « suis-je prêt(e) ? comment décider? » et de la feuille « se sentir prêt(e) à avoir des rapports sexuels ».

\section{PRÉPARATION :}

Passez en revue la liste de conditions et préparez-vous à gérer toutes les questions susceptibles d'être posées.
APERC̣U : Les élèves examinent les indicateurs de maturité, de conscience de soi, d'autoefficacité et d'aptitude à communiquer dont les jeunes ont besoin pour négocier une relation sexuelle confortable et sans risques. (Remarque : Avant cette activité, les élèves doivent avoir abordé les questions du comportement sexuel et de la contrainte sexuelle.)

OBJECTIFS : Aidez les élèves à identifier les compétences qui leur permettront de négocier une relation sexuelle confortable et sans risques; renforcer la réflexion abstraite.

\section{INSTRUCTIONS}

1 Présentez l'activité :

- Les jeunes décident si et quand ils deviennent sexuellement actifs selon différents critères. Certains attendent d'avoir un certain âge ; d'autres, d'être fiancés ou mariés. D’autres encore laissent plus ou moins quelqu'un d'autre prendre la décision pour eux. Beaucoup de jeunes considèrent leurs sentiments à l'égard de leur relation dans leur décision de commencer ou non à avoir des rapports sexuels.

- Il est difficile pour beaucoup de jeunes de déterminer s'ils sont prêts à franchir le pas. Cette activité vous aidera à comprendre l'importance de la maturité, de la conscience de soi et de la communication dans la préparation à l'activité sexuelle. Elle vous aidera aussi à déterminer ce qui importe le plus pour vous.
2 Invitez les élèves à s'organiser deux par deux et distribuez les instructions et les feuilles de travaille à chaque groupe.

- Considérez la liste proposée : elle énonce quelques-uns des sentiments et conditions qui peuvent être importants à l'assurance du confort et de la sécurité d'une relation sexuelle. Si une personne désire avoir des rapports sexuels et que la plupart de ces conditions sont remplies, elle aura plus de chances de se sentir à l'aise et en sécurité vis-à-vis de ces rapports.

- Si beaucoup de ces conditions font défaut dans la relation, la personne ne sera probablement pas à l'aise et courra un plus grand risque de rapports sexuels peu sûrs. 
3 Lisez les instructions de l'exercice à voix haute. Vérifiez que tout le monde comprend bien l'activité. Indiquez qu'ils vont cocher différents points en réponse à plusieurs questions. Il serait bon qu'ils soient d'accord sur les réponses, mais, à défaut, les deux peuvent cocher des points différents. Donnezleur 15 minutes pour discuter les questions et marquez leurs réponses.

4 Avec le groupe au complet, passez en revue les points 1 à 5 et demandez quelques réponses. Demandez si les élèves étaient généralement d'accord ou non avec leur partenaire

5 Pour le point 6, passez un peu plus de temps à examiner les réponses des élèves. Écrivez-les au tableau. Les questions suivantes peuvent guider la discussion :

- À quelles conditions les garçons semblent-ils donner la priorité ?

- Et les filles?

- Comment expliquez-vous les différences entre les priorités des garçons et celles des filles et qu'en pensez-vous?
6 Passez à la discussion des points 7 et 8, puis demandez:

- Certaines de ces conditions vous paraissent-elles sans importance, absolument inutiles parmi les facteurs de décision d'avoir ou non des rapports sexuels?

- Que vous choisissiez d'être sexuellement actifs à l'adolescence ou d'attendre jusqu'à l'âge adulte ou le mariage, l'établissement de ces conditions peut rendre votre expérience sexuelle plus confortable et moins risquée. [Dites au groupe si vous prévoyez de couvrir d'autres points relatifs à la question dans le cadre de votre programme. Par exemple, le volume 1 d'Un seul programme inclut des unités sur la communication, le corps et l'anatomie, la santé sexuelle et le VIH et les questions de genre.] 


\section{suis-je prêt(e) ? comment décider?}

Dans cet exercice, un jeune de votre âge essaie de décider s'il veut devenir sexuellement actif. Cette jeune personne veut prendre la bonne décision et se pose cette question : « Pour décider si je suis prêt(e) à avoir des rapports sexuels, quels sont les deux sentiments ou conditions les plus importants que je doive considérer? ॥ II ou elle pose la question à trois individus : son (sa) meilleur(e) ami(e), un conseiller (ou psychologue) et un sidéen.

\section{Suivez ces étapes :}

1 Lisez la liste énoncée sur votre feuille d'exercice.

2 Discutez et décidez comment chaque individu pourrait répondre à la question ci-dessus.

3 Meilleur(e) ami(e): Dans les cases correspondant aux deux sentiments ou conditions que votre meilleur(e) ami(e) indiquerait probablement comme les plus importants, dessinez un visage souriant ( (i)).

4 Conseiller : Cochez les cases correspondant aux deux sentiments ou conditions qu'un conseiller ou psychologue indiquerait probablement comme les plus importants $(\Omega)$.

5 Dans les cases correspondant aux deux sentiments ou conditions qu'une personne atteinte du sida indiquerait probablement comme les plus importants, tracez le symbole mondial de sensibilisation au sida $(\boldsymbol{X})$.

6 Discutez: Pensez-vous que certains de ces sentiments ou conditions sont plus importants pour les garçons que pour les filles ? Si oui, dessinez le schéma d'un garçon ou d'une fille, respectivement, en regard de tous les sentiments et conditions qui vous paraissent plus importants pour les garçons ou pour les filles.

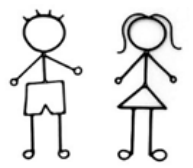

7 Pensez maintenant aux deux sentiments ou conditions que les jeunes omettent le plus souvent ou ont le plus de mal à considérer quand ils décident d'avoir des rapports sexuels pour la première fois. Tracez un visage triste dans les cases correspondantes ( (i)).

8 Quels autres sentiments ou conditions vous semblent importants à considérer avant d'avoir des rapports sexuels pour la première fois? 


\section{SE SENTIR « PRÊT(E) » À AVOIR DES RAPPORTS SEXUELS}

\begin{tabular}{|c|c|c|c|}
\hline Sentiment ou condition nécessaire & Dessinez ici & Sentiment ou condition nécessaire & Dessinez ici \\
\hline $\begin{array}{l}\text { Sentiment qu'on honore ses propres valeurs et celles de } \\
\text { son ou sa partenaire }\end{array}$ & & $\begin{array}{l}\text { Sentiment d'aise à lui demander ce qui lui donne du } \\
\text { plaisir }\end{array}$ & \\
\hline Sentiment d'être proche de l'autre personne & & $\begin{array}{l}\text { Sentiment d'aise à parler à l'autre de l'usage du } \\
\text { préservatif }\end{array}$ & \\
\hline Sentiment de respect mutuel entre les deux partenaires & & $\begin{array}{l}\text { Avoir des préservatifs (et/ou d'autres contraceptifs) et } \\
\text { savoir comment les utiliser }\end{array}$ & \\
\hline Sentiment de confiance entre les deux partenaires & & Connaissance de son propre état VIH & \\
\hline $\begin{array}{l}\text { Sentiment de décision prise ensemble et de désir mutuel } \\
\text { de rapports sexuels }\end{array}$ & & $\begin{array}{l}\text { Sentiment d'aise à parler de son propre état VIH et de } \\
\text { celui de son ou sa partenaire }\end{array}$ & \\
\hline Sentiment d'aise vis-à-vis de son propre corps & & $\begin{array}{l}\text { Sentiment mutuel de pouvoir dire à tout moment qu'on } \\
\text { veut arrêter }\end{array}$ & \\
\hline $\begin{array}{l}\text { Sentiment de confiance qu'on sait personnellement ce } \\
\text { qu'on aime sexuellement }\end{array}$ & & Espace privé & \\
\hline Sentiment d'attirance sexuelle vers l'autre personne & & $\begin{array}{l}\text { Sentiment de ne pas courir le risque d'être ridiculisé(e) } \\
\text { ou de subir l'opprobre par la suite }\end{array}$ & \\
\hline $\begin{array}{l}\text { Sentiment d'aise à dire à l'autre ce qu'on aime } \\
\text { sexuellement }\end{array}$ & & Autre? & \\
\hline
\end{tabular}




\section{vivre ensemble}

\section{DURÉE :}

45 minutes

\section{MATÉRIEL :}

Tableau noir + craie

\section{PRÉPARATION :}

Passez en revue la liste de pratiques ci-contre pour vous assurer qu'elles conviennent toutes à une brève discussion avec vos élèves. Veillez aussi à pouvoir définir simplement chacune de ces pratiques. Certaines peuvent être relativement rares dans votre contexte, mais les élèves peuvent en avoir entendu parler dans d'autres classes, au cinéma ou sur Internet.
APERC̣U : Les élèves se penchent sur l'évolution des coutumes relatives au mariage et à la structure familiale.

OBJECTIFS : Aider les élèves à clarifier leurs propres attentes et valeurs concernant leurs relations à long terme et à comprendre l'évolution des normes sociales au fil du temps ; renforcer la réflexion analytique et l'expression écrite.

\section{INSTRUCTIONS}

1 Copiez la liste suivante au tableau :

Mariage d'enfants

Relations ou mariages homosexuels

Couples ayant des rapports sexuels ou vivant ensemble avant d'être mariés.

\section{Mariage arrangé}

Homme battant sa femme si elle lui désobéit

Conseil conjugal

Familles étendues vivant ensemble

Hommes devenant des pères plus engagés

Polygamie (hommes ayant plusieurs épouses)

Divorce

Dot
2 Expliquez:

- Cette liste énumère certaines pratiques relatives à l'amour et au mariage dans différentes cultures. Certaines, comme le divorce, le mariage homosexuel ou l'âge minimum au moment du mariage, relèvent de la loi. D'autres, comme les familles étendues vivant ensemble, les couples vivant ensemble avant le mariage ou les modèles de paternité, sont moins formellement établies. Certaines reçoivent sans doute votre approbation, mais probablement pas toutes.

- Passez la liste en revue et sélectionnez une pratique qui va, selon vous, changer au cours de la prochaine génération, pour le meilleur ou pour le pire. 
3 Invitez les élèves à prendre leurs cahiers et à rédiger au moins une demi-page au sujet de ce changement. Posez ces questions pour stimuler la réflexion :

- Quelle pratique va, selon vous, changer au cours de la prochaine génération?

- Pensez-vous que le changement surviendra dans notre culture ou ailleurs?

- Anticipez-vous un changement d'attitudes? De fréquence de la pratique? De mode d'intervention de la pratique? Quoi d'autre?

- Pensez-vous que les lois qui régissent la pratique vont changer?

- N'oubliez pas d'indiquer si vous êtes d'accord avec le changement que vous prévoyez. Basezvous votre opinion sur votre expérience personnelle ou sur ce que vous avez vu ou entendu?

4 Pour chaque pratique de la liste, invitez un volontaire à lire ce qu'il ou elle en a écrit. Invitez ensuite un ou deux commentaires brefs. Faites varier le temps de discussion suivant que vous avez ou non un volontaire pour chaque pratique.
5 Réservez les 5 dernières minutes à la discussion :

- Qu'est-ce qui fait, selon vous, que des communautés entières ou même des pays changent leurs perspectives de l'amour et du mariage?

- D'où viennent les nouvelles idées et comment ces idées se répandent-elles? [Sondez : personnes qui choisissent de vivre autrement; médias ; échanges interculturels; évolution économique qui affecte le lieu de résidence des familles; mouvements politiques.]

- Imaginez que vous ayez un correspondant originaire d'une culture dont les pratiques sont très différentes des nôtres. En quittant la classe, réfléchissez à la manière dont vous pourriez lui décrire comment on trouve, ici, un partenaire à long terme, quels sont nos arrangements de vie commune et nos pratiques courantes de contraction ou dissolution d'un mariage. 


\section{ce que je recherche}

\section{DURÉE :}

Étapes 1-3: 40 minutes

Étape $4: 5$ minutes (au moins un jour avant les étapes 5-6)

Étapes 5-6: 40 minutes

\section{MATÉRIEL :}

Tableau noir + craie

\section{PRÉPARATION :}

Cette activité s'organise en deux parties (étapes 1-3 puis 4-6).

Choisissez de n'en présenter qu'une ou les deux. Si vous décidez de présenter les deux, veillez à achever l'étape 4 au moins un jour avant les étapes 5-6.

APERCUU : Les élèves listent les qualités de leur partenaire à long terme rêvé(e) et analysent leur liste dans la perspective du genre. Ils renforcent ce qu'ils ont appris en écrivant une lettre ou en composant une chanson pour leur partenaire imaginaire.

OBJECTIFS : Aider les élèves à identifier les caractéristiques qu'ils valorisent le plus dans un partenaire à long terme et à y réfléchir ; clarifier leurs valeurs et renforcer leur réflexion abstraite et leur expression écrite.

\section{INSTRUCTIONS}

1 Expliquez:

- Nous allons aujourd'hui considérer les qualités ou caractéristiques que vous recherchez dans un partenaire à long terme ou à vie.

- Dans votre cahier, écrivez au moins cinq qualités ou caractéristiques qui vous semblent importantes dans un conjoint ou un partenaire à long terme.

- Classez-les par ordre d'importance, en donnant le numéro 1 à la plus importante.

2 Invitez les élèves à former deux groupes, tous garçons d'un côté et toutes filles de l'autre (sauf si la classe n'est pas mixte). Si la classe est nombreuse, formez deux groupes de chaque sexe. Expliquez:

- Comparez votre liste à celles d'autres membres de votre groupe. Identifiez les qualités ou caractéristiques le plus souvent classées parmi les plus importantes (rangs 1 et 2). [Plusieurs personnes peuvent utiliser différents mots pour une même qualité (bon/gentil ou drôle/amusant, par exemple). Circulez et offrez votre aide.]
- Invitez un membre de chaque groupe à venir au tableau et à y écrire les trois ou quatre qualités les plus importantes identifiées par le groupe. Marquez si la liste est féminine ou masculine.

3 Passez chaque liste en revue et animez la discussion, en vous aidant de ces questions :

- Que remarquez-vous au sujet de ces deux listes?

- En quoi sont-elles similaires?

- En quoi sont-elles différentes?

- Pourquoi ces différences?

- Les filles ont-elles des questions à poser aux garçons au sujet de leur liste?

- Et les garçons au sujet de celle des filles ?

- Remarquez-vous des stéréotypes de genre dans les listes? Si oui, lesquels? 
4 (Cette étape doit être accomplie au moins un jour avant les étapes 5-6. Si possible, écrivez la tâche au tableau.) Expliquez la tâche de rédaction. Clarifiez si elle devra être accomplie en classe ou comme devoir.

- Imaginez le type de personne avec qui vous aimeriez passer le restant de votre vie ou avoir comme partenaire à long terme.

- Écrivez une lettre, un poème ou une chanson à cette personne imaginaire.

- Parlez de ce que vous recherchez dans cette personne et dans une relation à long terme. Indiquez vos attentes, comment vous aimeriez être traités et ce que vous ne pourriez tolérer.

- Si vous pensez que vous préféreriez ne pas avoir de relation à long terme dans votre vie, écrivez plutôt un texte ou une chanson à ce sujet.

- Demain (ou lors d'une prochaine session), vous aurez l'occasion de lire ou d'interpréter ce que vous avez écrit.
5 Fixez la date où les élèves pourront présenter leurs lettres, poèmes et chansons. Si le temps disponible ne suffit pas, invitez seulement les volontaires à présenter leur texte. Vous pouvez aussi inviter les élèves à vous remettre leur travail pour une lecture anonyme.

6 Réservez 10 minutes à la discussion, avec l'aide des questions suivantes :

- Qu'avez-vous entendu qui vous a particulièrement touchés?

- Comment les différences d'attente des garçons et des filles affectent-elles les relations?

- Comment expliquez-vous ces différences? Comment certaines personnes en arrivent-elles à donner la priorité à certaines caractéristiques ?

- Est-il nécessaire, pour tout le monde, d'établir une relation à long terme pour être heureux ?

- Pourquoi est-il important de réfléchir maintenant au type de relation que vous pourriez désirer (ou non)? 


\section{l'amour à la dérive}

\section{DURÉE :}

Étapes 1-3: 30 minutes

Étapes 4-6:50 minutes

\section{MATÉRIEL :}

Tableau noir + craie ; quatre récits (extraits des « études de cas de relations à la dérive $»$ ), reproduits chacun sur une feuille différente.

\section{PRÉPARATION :}

Sélectionnez parmi les études proposées les quatre situations les plus appropriées pour vos élèves. Au besoin, adaptez-les et copiez-les chacune sur une feuille séparée. Renseignez-vous sur l'endroit où peut s'adresser une personne qui cherche de l'aide concernant une relation à la dérive ou sujette à un risque de violence au sein d'une relation. (Voir la dernière question de l'étape 6.)
APERCUU : Les élèves achèvent le récit de relations à la dérive et réfléchissent aux causes de rupture. (Remarque : Avant cette activité, les élèves doivent avoir été informés du droit de ne pas subir de violences dans les relations intimes.)

OBJECTIFS : Apprendre aux élèves à identifier les stratégies de résolution de problèmes relationnels et de rupture ; identifier les comportements qu'ils ne toléreraient pas dans une relation; clarifier leurs valeurs concernant la dignité dans les relations ; favoriser l'apprentissage collaboratif et l'expression en public.

\section{INSTRUCTIONS}

1 Expliquez:

- Nous allons explorer aujourd'hui les problèmes qui peuvent affecter les relations.

- Quels sont quelques problèmes souvent rencontrés dans les relations amoureuses? [Écrivez les réponses au tableau. Par exemple: ingérence familiale, communication, attentes ou engagement inégaux, jalousie et conflits d'argent ou de pouvoir de décision.]

2 Divisez la classe en quatre groupes. Expliquez:

- Je vais donner à chaque groupe le début de l'histoire d'une relation. Chacun devra décider de la manière d'achever l'histoire : que doit faire le couple dans les limites du possible?

- Préparez-vous à interpréter votre conclusion dans un sketch de moins de 5 minutes. Imaginez et interprétez la conversation du couple.
- Deux personnes, dans chaque groupe, vont jouer le rôle des deux partenaires. Les autres membres du groupe peuvent jouer d'autres rôles (d'autres membres de la famille, par exemple).

3 Donnez à chaque groupe un début de récit et le temps de se préparer.

4 Invitez un groupe à interpréter sa conclusion, puis donnez quelques minutes de discussion à la classe :

- Quels sont les problèmes ou le conflit apparents dans ce couple?

- Comment ont-ils abordé leurs problèmes?

- Que pensez-vous de la manière dont le groupe a résolu la situation ? L'issue est-elle réaliste?

- Auriez-vous d'autres suggestions? 
5 Procédez de même aux autres présentations. Prévoyez environ 10 minutes par groupe (sketch + discussion).

6 Pour conclure, animez la discussion en vous aidant des questions suivantes :

- Quels sont les signes avant-coureurs d'une relation à la dérive?

- Quelles circonstances gardent parfois une personne dans une relation à la dérive ou violente? (Pensez enfants, nécessité économique, honte du divorce.)

- Il arrive que des partenaires mettent fin à leur relation, même s'ils sont mariés. Traite-t-on les hommes et les femmes divorcés de la même manière que les gens mariés? [Si non, demandez: pourquoi sont-ils vus différemment? Qu'en pensezvous?]

- Quelles circonstances ou quel comportement vous mèneraient-ils à la rupture?

- Où une personne qui court le risque d'une relation violente peut-elle obtenir de l'aide? Et où s'adresser pour obtenir aide et conseil au sujet d'une relation à la dérive? 


\section{études de cas de relations à la dérive}

Adey et Beto sortent ensemble depuis le lycée et sont aujourd'hui inscrits dans deux universités différentes. Ils sont tous deux de retour chez eux pour les vacances. Hier soir, ils sont sortis avec d'autres amis. Beto a fait quelques remarques blessantes au sujet d'Adey. À son regard contrarié, il a dit qu'il plaisantait. Plus tard, Adey a interrogé Beto sur une femme qu'il a mentionnée plusieurs fois. Il lui avoue avoir eu des rapports sexuels avec elle...

Cala et Daoud sont mariés depuis un an. Ils se disputent souvent, mais ils n'en étaient jamais venus aux mains, jusqu'il y a environ un mois. Ils se sont férocement disputés au sujet de la famille de Cala et Daoud l'a giflée une ou deux fois. Contrit, il lui a acheté un cadeau le lendemain, lui promettant que cela ne se reproduirait jamais. Hier soir pourtant, il l'a giflée et elle s’est réveillée, ce matin, l’œil noir et meurtri ...

Elena et Fritz sortent ensemble depuis plusieurs années. Au début, ils passaient de si bons moment ensemble — ils riaient, ils parlaient de tout et ils avaient une intense relation physique. Mais il semble aujourd'hui qu'ils soient trop habitués l'un à l'autre. Ils regardent presque toujours la télé et ne se parlent pratiquement plus. Même leur vie sexuelle en a pris un coup. Elena se demande : «Où est cet amour que j'avais pour lui ?»

Grete et Henri ont commencé à sortir ensemble il y un an. Grete a 18 ans et commence ses études à l'université. Henri en a 25 et travaille. Grete est vraiment passionnée par ses études et heureuse à l'idée de se faire de nouveaux amis, d'étudier et de profiter de sa vie d'étudiante. Henri se sent prêt à se ranger et à fonder une famille. Il pense qu'il aimerait épouser Grete. Il le lui a dit récemment et a été stupéfait de sa réaction. Elle l'a regardé comme s'il était fou et lui a dit : «Je commence à peine mes études. Je veux profiter de ma vie d'étudiante, me concentrer sur mes études et certainement pas me marier!» 


\section{études de cas de relations à la dérive}

Ivan et Josefa sont ensemble depuis plus d'un an. Ils travaillent tous les deux et aiment sortir avec leurs amis. Hier soir, ils sont allés dans un club. Josefa a parlé, ri et dansé avec ses amies. Ivan a dansé avec plusieurs personnes et eu une conversation intéressante avec une femme qu'il venait de rencontrer. Il pensait qu'ils avaient tous les deux passé une bonne soirée mais, en rentrant, Josefa était fâchée : « Je t’ai vu regarder cette femme. Ne me dis pas que tu n'étais pas intéressé ! Et ta façon de danser ! Comme si tu essayais d'attirer l'attention de tout le monde !»

Kiki, étudiante à l'université, travaille à mi-temps mais ne gagne pas beaucoup. Lisa occupe, elle, un bon emploi et gagne bien sa vie. Elle paie donc la plupart de leurs dépenses. Kiki et Lisa ont convenu que, dans la mesure du possible, Kiki ferait le marché ou contribuerait au loyer, mais c'est plutôt rare. Quand Kiki s'achète des vêtements, elle va dans les boutiques chères. Lisa ne voit pas l'utilité de dépenser autant d'argent. Voici quelques jours, Kiki a été payée pour un travail de vacances. Hier, elle a dépensé la moitié pour acheter un ordinateur sans demander l'avis de Lisa. Quand elle le ramène à la maison, Lisa ne contient pas sa colère.

Mika et Nobu sont mariés et ont trois enfants, âgés de un, trois et six ans. Les règles de Mika sont en retard. Elle se rend à la clinique et apprend qu'elle est enceinte de cinq semaines. La nouvelle la désespère. Ils s'en sortent à peine, Nobu et elle, et ont si peu d'argent. La colère la prend aussi. Elle soupçonne être tombée enceinte la nuit où Nobu est rentré ivre et n’a pas utilisé le préservatif. Elle dit à Nobu qu’elle veut se faire avorter. Nobu est stupéfait. Il lui dit : « Je sais que ce n'est pas facile, mais je ne veux pas que tu fasses ça..." 


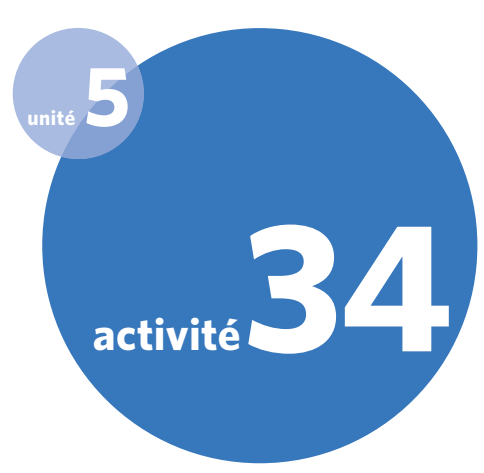

DURÉE :

45-60 minutes

\section{MATÉRIEL :}

Une copie des deux feuilles de travaille (« auto-évaluation » et « analyse de réponses ») par élève, si possible.

\section{PRÉPARATION :}

Photocopiez les feuilles à distribuer, si possible. Préparezvous sinon à les lire.

\section{ai-je dit cela ? différents styles de communication}

APERCUU : Les élèves analysent différentes manières de répondre verbalement à une situation inconfortable et font la distinction entre la communication agressive, assurée et passive. Ils remplissent un questionnaire sur leur style de réponse aux situations où ils ne se sentent pas à l'aise.

OBJECTIFS: Aider les élèves à mieux comprendre différents styles de communication et à formuler leurs réponses assurées; améliorer leur réflexion analytique et leurs aptitudes au dialogue.

\section{INSTRUCTIONS}

1 Présentez l'activité :

- Nous allons parler aujourd'hui des différents styles de communication.

- En particulier, quand on se sent mal à l'aise dans une situation, on ne communique pas toujours clairement et comme on le voudrait.

- Pourquoi est-il parfois difficile d'exprimer ses désirs de la meilleure façon possible? [Sondez et écrivez les réponses suivantes au tableau :]

Je ne sais pas comment m'exprimer clairement.

J'ai peur de ce que l'autre personne pensera de moi si je ne suis pas d'accord avec elle.

Je crois que je ne serai de toute façon pas écouté(e) et que mes paroles ne comptent pas.

J'ai peur de me mettre en colère.

Il m'est plus facile d'intimider l'autre.
- Nous allons commencer par examiner la différence entre la communication agressive, passive et assurée. Vous remplirez ensuite un questionnaire sur vos propres styles de réponse dans les situations où vous vous sentez mal à l'aise.

- Quelle est, selon vous, la différence entre la communication passive et la communication agressive? Qu'entend-on par communication assurée? [Sondez : être honnête; être direct; exprimer ses sentiments et ses besoins; utiliser un langage corporel assuré; défendre son point de vue; communiquer clairement mais sans agressivité.] Qu'est-ce que la communication indirecte? [Sondez: ne pas être totalement passif, mais sans être direct non plus.] 
2 Invitez les élèves à s'organiser deux par deux et distribuez (ou lisez à voix haute) la feuille « Analyse de réponses ». Expliquez :

- Avec votre partenaire, lisez la description de la situation de conflit présentée dans la colonne de gauche. Lisez ensuite les réponses énoncées dans la colonne suivante. Pour chacune, cochez s'il s'agit d'une réponse agressive, passive ou assurée. Pour le conflit $\mathrm{n}^{\circ} 5$, imaginez un autre conflit et les différents types de réponse.

3 Passez en revue chaque conflit et invitez les élèves à vous donner les réponses correctes, puis à commenter l'effet de la réponse agressive par rapport à celle assurée. (Clé : Les réponses $\mathrm{a}, \mathrm{f}$, $\mathrm{i}$, et $\mathrm{k}$ sont agressives. Les réponses $\mathrm{c}$, $\mathrm{d}$ et $\mathrm{h}$ sont passives. Les réponses $b, e, g$ et $j$ sont assurées.)
4 Distribuez la feuille d'auto-évaluation et invitez les élèves à la remplir individuellement. (Ou bien écrivez les énoncés et les réponses possibles au tableau, lisez-les à haute voix et invitez les élèves à répondre par écrit.)

5 Pour conclure, posez les questions suivantes:

- Qu'avez-vous appris au sujet de votre propre style de communication?

- Serait-il utile d'apprendre à communiquer de manière assurée? 


\section{auto-évaluation : comment je communique quand je me sens mal à l'aise}

Quand je me sens mal à l'aise vis-à-vis d'un garçon, j'ai tendance à être :

$\square$ Passif(ve) $\square$ Sûr(e) de moi, respectueux(se) $\square$ Agressif(ve) $\square$ Indirect(e) ou manipulateur(trice)

Quand je me sens mal à l'aise vis-à-vis d'une fille, j'ai tendance à être :

$\square$ Passif(ve) $\quad \square$ Sûr(e) de moi, respectueux(se) $\quad \square$ Agressif(ve) $\square$ Indirect(e) ou manipulateur(trice)

Quand je me sens mal à l'aise vis-à-vis d'un homme adulte, j'ai tendance à être :

$\square$ Passif(ve) $\quad \square$ Sûr(e) de moi, respectueux(se) $\quad \square$ Agressif(ve) $\square$ Indirect(e) ou manipulateur(trice)

Quand je me sens mal à l'aise vis-à-vis d'une femme adulte, j'ai tendance à être :

$\square$ Passif(ve) $\quad \square$ Sûr(e) de moi, respectueux(se) $\quad \square$ Agressif(ve) $\square$ Indirect(e) ou manipulateur(trice)

Quand je me sens mal à l'aise vis-à-vis de quelqu'un que je ne perçois pas comme mon égal, j'ai tendance à être :

$\square$ Passif(ve) $\quad \square$ Sûr(e) de moi, respectueux(se) $\quad \square$ Agressif(ve) $\quad \square$ Indirect(e) ou manipulateur(trice)

$\square$ Je ne peux pas répondre car je perçois tout le monde comme mon égal.

Pour moi, l'expression de sentiments de colère est :

$\square$ Relativement facile (mais je réagis parfois de manière agressive)

$\square$ Relativement facile (et je ne réagis jamais de manière agressive ou violente)

$\square$ Ni facile, ni difficile

$\square$ Plutôt ou même très difficile

Pour moi, l'expression de sentiments de faiblesse ou de vulnérabilité est :

$\square$ Relativement facile (mais je réagis parfois de manière agressive)

$\square$ Relativement facile (et je ne réagis jamais de manière agressive ou violente)

$\square$ Ni facile, ni difficile

$\square$ Plutôt ou même très difficile 


\section{ANALYSE DE RÉPONSES}

\begin{tabular}{|c|c|c|c|c|}
\hline CONFLIT & RÉPONSE & Agressive & Passive & Assurée \\
\hline \multirow{3}{*}{$\begin{array}{l}\text { Un garçon et une fille sont sur le } \\
\text { point d'avoir leurs premiers rapports } \\
\text { sexuels ensemble mais ils n'ont pas } \\
\text { de préservatifs. L'un d'entre eux dit: }\end{array}$} & a. Pour autant que je sache, tu es déjà séropositif(ve), connard(e)! & & & \\
\hline & $\begin{array}{l}\text { b. Moi aussi je veux faire l'amour avec toi, mais seulement si on } \\
\text { utilise le préservatif. Je sais où on peut en trouver. }\end{array}$ & & & \\
\hline & c. Bon, d'accord. & & & \\
\hline \multirow{3}{*}{$\begin{array}{l}2 \text { Un garçon n'est pas content que } \\
\text { sa copine ait parlé à un autre à une } \\
\text { soirée. Il dit: }\end{array}$} & d. Pas très amusante, la soirée. & & & \\
\hline & $\begin{array}{l}\text { e. Pendant que tu parlais à ce gars, j'ai eu l'impression que tu } \\
\text { préférerais peut-être être avec lui. Ça m'a mis mal à l'aise. }\end{array}$ & & & \\
\hline & $\begin{array}{l}\text { f. Je t'ai vue ! Comment oses-tu parler à ce type alors que tu étais } \\
\text { avec moi ? }\end{array}$ & & & \\
\hline \multirow{3}{*}{$\begin{array}{l}3 \text { Un garçon invite une fille à sortir avec } \\
\text { lui. Elle ne veut pas. Elle dit : }\end{array}$} & g. Merci, mais ça ne me dit rien. Désolée. & & & \\
\hline & h. Euh... D'accord. & & & \\
\hline & $\begin{array}{l}\text { i. Je ne sortirais jamais avec quelqu'un comme toi. Ne m'approche } \\
\text { pas! }\end{array}$ & & & \\
\hline \multirow{2}{*}{$\begin{array}{l}4 \text { Une fille en veut à son amie Lulu de } \\
\text { l'avoir taquinée. Elle dit: }\end{array}$} & j. Lulu, ça me dérange que tu te sois moquée de moi. & & & \\
\hline & $\begin{array}{l}\text { k. Lulu, tu n'es qu'une imbécile. Tu vas voir, je vais me mettre à } \\
\text { raconter des histoires sur toi ! }\end{array}$ & & & \\
\hline \multirow{3}{*}{$\begin{array}{l}5 \text { Imaginez une autre situation de } \\
\text { conflit. Résumez-la ici puis écrivez } \\
\text { les réactions des types demandés } \\
\text { dans les colonnes de droite. }\end{array}$} & & $x$ & & \\
\hline & & & X & \\
\hline & & & & $x$ \\
\hline
\end{tabular}




\title{
écoute active
}

\author{
APERC̣U : Les élèves s'exercent aux comportements qui enrichissent (ou entravent) la \\ communication.
}

OBJECTIFS : Aider les élèves à adopter de bons comportements de communication.

\section{INSTRUCTIONS}

1 Donnez aux volontaires un aide-mémoire pour chaque sketch. Si nécessaire, laissez-les se préparer pendant que vous présentez l'étape 2 .

2 Présentez le sujet au reste de la classe. Expliquez:

- On dit que la communication est bidirectionnelle. Quand une personne parle, que fait l'autre? [Elle écoute.] L'écoute fait-elle donc partie de la communication? [Oui.] Nous allons explorer aujourd'hui ce que nous communiquons par notre écoute et découvrir l'immense différence, au profit de la communication, de «l'écoute active ».

- Nous allons commencer par une démonstration. Prenez vos cahiers. Observez la personne qui écoute et prenez note de chaque comportement qui vous paraît contraire à une communication efficace.

3 Invitez les volontaires à interpréter le sketch de communication « négative» (sketch 1$)$.
4 Posez les questions de réflexion suivantes:

- Que pensez-vous de cette conversation? Vous a-telle paru satisfaisante et efficace?

- Quels comportements d'écoute verbaux et non verbaux avez-vous observés? [Au tableau, écrivez les comportements négatifs mentionnés. Sondez:]

\section{Comportements non verbaux :}

Froncement des sourcils

Air dédaigneux, roulement d'yeux

Hochement de la tête comme pour dire non

Regard ou mouvement d'éloignement, signes

d'ennui, indifférence, bâillements

\section{Comportements verbaux :}

Interruption de l'interlocuteur, impatience, indication que l'interlocuteur parle trop longtemps Expression de jugements négatifs de l'interlocuteur ( "Tu as tort»ou «C'est idiot»).

- Demandez à la personne «non écoutée » l'effet que cela fait, de ne pas être écouté. 
5 Invitez les volontaires à interpréter le sketch de communication « positive» (sketch 2$)$.

6 Posez les questions de réflexion suivantes:

- Que pensez-vous de cette conversation? Vous a-telle paru plus satisfaisante?

- Quels comportements verbaux et non verbaux ont rendu cette conversation plus productive? [Au tableau, écrivez tous les comportements positifs mentionnés. Sondez:]

\section{Comportements non verbaux :}

Maintien du contact oculaire

Signes affirmatifs de la tête

Inclination légère vers l'interlocuteur, en signe

d'écoute

Sourire ou tape amicale dans le dos

\section{Comportements verbaux :}

Demande de clarification pour s'assurer de bien comprendre

Indication d'intérêt (en disant, par exemple:

"Je suis tout oreilles, continue... .")

Validation des sentiments de l'interlocuteur (par exemple: "Je comprends ce que tu ressens")

Validation des idées de l'interlocuteur (par exemple: "Oui, en effet »)

Absence de commentaires qui donnent à

l'interlocuteur l'impression d'être incompétent

Remerciement de l'interlocuteur pour sa confiance à partager ce qu'il avait à dire
7 Invitez les élèves à s'organiser par trois pour pratiquer l'écoute active. Expliquez :

- Dans chaque groupe, une personne va parler pendant 2 minutes. Quand viendra votre tour, parlez de quelque chose qui vous inquiète ou vous dérange. Choisissez pour cet exercice un sujet qui n'est pas confidentiel et dont vous pouvez parler à l'aise avec vos camarades de de classe. Parlez de votre espoir, votre colère, votre inquiétude, votre humiliation ou votre anxiété. Si vous ne savez pas de quoi parler, demandez à votre interlocuteur de voter pour vous à la présidence du Conseil des Jeunes.

- Un autre membre du groupe pratiquera l'écoute active tandis que le troisième observera et fera la critique, principalement de la personne qui écoute, mais aussi peut-être de celle qui parle. Au bout de deux minutes, vous changerez les rôles, puis, de nouveau, une troisième fois, pour que chacun ait l'occasion de jouer chaque rôle.

\section{$81^{\text {er }}$ tour :}

- Ceux dont le tour est de parler, commencez à dire à votre interlocuteur ce qui vous préoccupe.

- [Après 2 minutes :] Arrêtez. Observateurs, vous avez 60 secondes pour donner vos commentaires. 
9 Après 1 minute : $2^{\mathrm{e}}$ tour :

- Au tour de ceux qui ont parlé d'écouter, de ceux qui ont écouté d'observer et de ceux qui ont observé, de parler.

- [Après 2 minutes :] Arrêtez. Observateurs, 1 minute de commentaires.

10 Après 1 minute : $3^{\mathrm{e}}$ tour. Pour ce tour, peut-être voudrez-vous écrire au tableau cette citation du médecin-écrivain Karl Menninger, pour inspirer la réflexion : «L'écoute est une qualité magnétique singulière, une force créative. Les amis qui nous écoutent sont ceux dont nous nous rapprochons. Leur écoute nous crée, nous ouvre et nous épanouit. »

- Changez de tour une troisième et dernière fois.

- [Après 2 minutes :] Arrêtez. Observateurs, à vous.

11 Réservez 10 minutes à la discussion, en vous inspirant de ces questions :

- Que ressentez-vous quand vous parlez et que votre interlocuteur ne vous écoute pas bien?

- Que se passe-t-il quand les deux interlocuteurs ont un véritable conflit et s'énervent tous deux? Est-il plus ou moins facile de communiquer?
- Différents styles de communication ont été observés chez les filles par rapport aux garçons. Qu'apprend-on aux filles et aux femmes sur la façon de s'exprimer quand quelque chose les dérange?

- Et aux hommes et garçons? Est-il possible d'apprendre à communiquer de manière non violente ni agressive?

- Comment pensez-vous que l'agressivité et la violence affectent la communication entre les adolescents des deux sexes dans une relation intime?

- Que peut-on faire pour améliorer la communication entre les deux sexes, surtout en cas de conflit? Combien d'entre vous ont pu donner une critique constructive, quand vous étiez observateurs?

- [S'il vous reste du temps :] Que pensez-vous de la citation que j'ai écrite au tableau?

- Réfléchissez à une chose que vous aimeriez améliorer dans votre style de communication. Exercez-vous ce soir et pendant la semaine. Remarquez aussi comment les autres vous écoutent. 


\section{aide-mémoire - sketch de « mauvaise écoute » (1) :}

L'un(e) d'entre vous doit « parler » et l'autre, « écouter ».

SI VOUS PARLEZ : Développez votre discours autour d'une situation imaginaire : (1) une situation injuste qui vous est (prétendument) arrivée ; ou (2) un projet ou une idée folle qui vous passionne. Pour le sketch, exprimez très clairement votre expérience ou vos idées et vos sentiments. Vous allez passer plusieurs minutes à en parler à votre interlocuteur et à essayer d'être entendu(e). Prévoyez de parler pendant deux à trois minutes.

Réagissez à la manière dont votre partenaire vous écoute. S'il vous semble qu'il ou elle ne vous écoute pas, montrez votre frustration, haussez la voix ou réagissez de la manière qui vous semble naturelle dans ce genre de situation. Si l'autre vous écoute attentivement et avec respect, comportez-vous en conséquence.

SI VOUS ÉCOUTEZ : Vous êtes un exemple de MAUVAISE ÉCOUTE. Démontrez-en les caractéristiques. Votre tâche est d'adopter autant de comportements verbaux et non verbaux négatifs que possible dans la liste ci-dessous :

Comportements non verbaux:

- Froncement des sourcils

- Air dédaigneux, roulement d'yeux

- Hochement de la tête comme pour dire non

- Impatience - regard ou mouvement d'éloignement, signes d'ennui, indifférence, bâillements

Comportements verbaux :

- Interruption de l'interlocuteur, réorientation de la conversation sur vous-même ou changement de sujet

- Expression de jugements négatifs de l'interlocuteur (tels que «Que non ! » ou « C'est idiot. ») 


\section{aide-mémoire - sketch de « bonne écoute » (2) :}

L'un(e) d'entre vous doit « parler » et l'autre, « écouter ».

SI VOUS PARLEZ : (Mêmes instructions que pour le sketch 1) : Développez votre discours autour d'une situation imaginaire : (1) une situation qui vous est (prétendument) arrivée; ou (2) un projet ou une idée folle qui vous passionne. Pour le sketch, exprimez très clairement votre expérience ou vos idées et vos sentiments. Vous allez passer plusieurs minutes à en parler à votre interlocuteur et à essayer d'être entendu(e). Prévoyez de parler deux à trois minutes.

Réagissez à la manière dont votre partenaire vous écoute. S'il vous semble qu'il ou elle ne vous écoute pas, montrez votre frustration, haussez la voix ou réagissez de la manière qui vous semble naturelle dans ce genre de situation. Si l'autre vous écoute attentivement et avec respect, comportez-vous en conséquence.

SI VOUS ÉCOUTEZ : Vous êtes un exemple d'ÉCOUTE ACTIVE. Démontrez-en les caractéristiques. Votre tâche est d'adopter autant de comportements verbaux et non verbaux positifs que possible dans la liste ci-dessous :

Comportements non verbaux:

- Maintien du contact oculaire

- Signes affirmatifs de la tête

- Inclination légère vers l'interlocuteur, en signe d'écoute

- Sourire ou tape amicale dans le dos

Comportements verbaux:

- Demande de clarification pour vous assurer de bien comprendre

- Indication d'intérêt (en disant, par exemple : « Je suis tout oreilles, continue... »)

- Validation des sentiments de l'interlocuteur (par exemple : « Je comprends ce que tu ressens ».)

- Validation des idées de l'interlocuteur (par exemple : « Oui, en effet ».)

- Absence de commentaires qui donnent à l'interlocuteur l'impression d'être incompétent

- Remerciement de l'interlocuteur pour sa confiance à partager ce qu'il ou elle avait à dire 


\section{pratiquer la communication affirmative}

APERCU : Les élèves analysent des comportements assurés, parlent des implications culturelles et pratiquent la communication affirmative dans des situations d'abus de droits.

OBJECTIFS : Aider les élèves à communiquer, quand il le faut, de manière plus assurée ; renforcer leurs aptitudes au dialogue.

\section{INSTRUCTIONS}

1 Commencez par ces questions-guides :

- Nous allons parler aujourd'hui de la communication affirmative. Il est parfois difficile de dire clairement ce qu'on veut (qu'on désire par exemple être considéré pour un poste de leadership à l'école). Il est parfois difficile aussi de dire ce qu'on ne veut pas (manger quelque chose que quelqu'un a préparé pour vous, par exemple).

- Vous souvenez-vous d'une situation où vous ne saviez pas trop comment exprimer vos désirs clairement? [Remarquez si les filles ou les garçons semblent davantage concernés.]

- Il est parfois difficile de se défendre, même dans les situations importantes, ou face à de vraies conséquences physiques ou affectives. Il peut être utile de s'exercer à communiquer de manière respectueuse mais tout aussi assurée.

- Prenez vos cahiers. Je vais lire une série de questions ou énoncés. Pour chacun, écrivez une réponse ferme et assurée mais pas agressive. Rédigez vos réponses en phrases complètes.

- Le premier énoncé est : « Dites-moi pourquoi je devrais voter pour vous. » élèves à arriver à une réponse appropriée, telle que: "Je crois que je suis la candidate la plus qualifiée. J'espère pouvoir compter sur votre soutien. "Veillez à utiliser des phrases complètes.]

2 Répétez pour chaque énoncé (voir la liste).

3 Pour conclure, discutez :

- S'exprimer de manière assurée peut paraître impoli ou contraire à la culture de certains. Dans quelle situation convient-il de donner une réponse ferme et assurée même si cela paraît impoli? [Sondez : quand vos droits sont violés.]

- Les filles qui s'affirment avec assurance sont-elles traitées comme les garçons qui le font ? Lassurance d'une personne est-elle vue différemment si cette personne est issue d'une minorité ethnique ou raciale? Comment ces préjugés limitent-ils la capacité de se défendre?

Devoir : Exprimez-vous au moins une fois de manière plus assurée durant les prochains jours. Documentez la situation et vos impressions par écrit.
- Donnez-moi une réponse assurée. [Aidez les
DURÉE :

30-40 minutes

\section{MATÉRIEL :}

Votre copie de la « liste d'énoncés et de réponses types ».

\section{PRÉPARATION :}

Lisez les énoncés et les réponses types. Au besoin, modifiez-les pour les adapter à votre culture. 


\section{liste d'énoncés et de réponses assurées types}

Lisez les énoncés suivants comme invites à une réponse assurée. Des réponses types sont proposées au cas où vos élèves n'arriveraient pas à une réponse appropriée.

1 Invite : «Tu me laisses copier tes réponses à l'interro ? Le prof n'en saura rien. » Réponse type : «Désolée. Je ne crois pas au partage de réponses. »

2 Invite: Vous voyez votre chef d'atelier et vous voulez lui demander une augmentation. Réponse type : «Chef, j'aimerais vous parler d'une augmentation que je pense avoir méritée. »

3 Invite: Dites à votre père que vous désirez continuer à aller à l'école l'an prochain, contre son gré. Réponse type : «Papa, je respecte ton opinion, mais il est extrêmement important pour moi de poursuivre mes études. J'aimerais discuter mes raisons avec toi. »

4 Invite : «Pourquoi n'aimes-tu pas le sport comme tout le monde? Tu es malade ou quoi ?» Réponse type: « À chacun ses goûts. Moi, j'aime lire et la musique. »

5 Invite : « Viens avec nous derrière la vieille usine. La police ne nous y dérange jamais. » Réponse type : « Non merci. Ça ne m'intéresse pas. »

6 Invite : « Je suis le maire. On me dit que vous aimeriez utiliser un espace municipal pour votre club après l'école ? 》 Réponse type: « Oui, monsieur le Maire. Nous sommes un groupe responsable et nous respecterons l'espace. Voulezvous que je vous présente nos projets?»

7 Invite : « Si vous pouvez convaincre vos deux camarades de classe d'y travailler avec vous, nous vous laisserons peindre votre peinture murale. » (Adressez votre réponse à vos camarades de classe.)

Réponse type : « Nous avons la permission de peindre sur le mur si deux autres élèves se joignent à nous. Ça vous intéresse?»

8 Invite : « Nous allons en excursion. Où penses-tu que nous pourrions aller? » Réponse type : « J'aimerais aller à . Je pense que ce serait intéressant pour les autres aussi. »

9 Invite: « Le nouveau gars de l'autre classe vient par ici. Faisons-lui un croc-en-jambe. » Réponse type : «Laisse-le tranquille. II ne nous a rien fait. »

10 Invite : Revenez maintenant à la situation dont vous vous êtes souvenus en début d'activité, quand je vous ai demandé de penser à une situation où vous auriez voulu exprimer vos désirs plus directement. Rédigez ce que vous auriez pu dire dans cette situation. Vous ne devrez pas partager votre réponse. 


\section{5 étapes pour communiquer au sujet d'un conflit}

APERCUU : Les élèves apprennent un modèle en cinq étapes pour communiquer clairement et avec respect au sujet d'un conflit interpersonnel. (Remarque : Cette activité favorise le développement d'aptitudes de communication plus avancées.)

OBJECTIFS : Aider les élèves à développer leurs aptitudes de communication dans une situation de conflit intime ; renforcer la réflexion analytique et l'aptitude au dialogue.

\section{INSTRUCTIONS}

1 Expliquez:

- Nous allons découvrir et pratiquer aujourd'hui une technique de communication au sujet d'un conflit, d'abord ensemble, puis par groupes de deux pour que chacun ait l'occasion de participer.

- Voici, d'abord, une situation imaginaire que nous allons aborder tous ensemble. Ron pense que Kim le ridiculise devant leurs amis. Il est furieux et va confronter Kim.

2 Au tableau, écrivez la première invite ( $Q$ QUAND TU ») et discutez:

- Quel est le comportement particulier qui dérange Ron ? Plutôt que de lancer une accusation générale du type "Tu n'es pas une vraie amie... », parlez du comportement spécifique en cause. [Sondez: "Quand tu te moques de moi ...» ou "Quand j'ai appris que tu t'étais moquée de moi ...»Au tableau, complétez l'invite en écrivant le comportement, comme énoncé par le groupe.]

- Pourquoi est-il important de communiquer spécifiquement le comportement dérangeant?
- Si vous n'êtes pas sûrs de votre perception du comportement, reconnaissez-le, en disant par exemple : «J'ai peut-être tort, mais j’ai entendu dire que tu te moquais de moi ». Pourquoi peut-il être important de permettre l'ajustement de votre perception de la situation?

3 Écrivez la seconde invite: «J'IMAGINE QUE et discutez:

- Bien qu'extrêmement importante, cette étape est souvent omise. Elle nous force à réfléchir à nos réponses et à nos réactions.

- Que peut imaginer ou commencer à penser Ron ? "Quand tu te moques de moi, j'imagine (je commence à penser) ...» que tu te fiches de mes sentiments. Ou bien: que ce n'est pas la première fois que tu me casses du sucre sur le dos. Ou :

que tu ne dois pas te sentir bien dans ta peau. $\mathrm{Ou}$ :

que tout le monde rit de moi.

Ou bien encore?

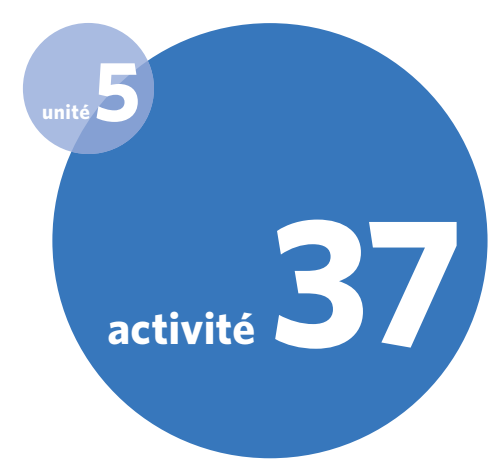

DURÉE :

45-60 minutes, suivant le degré d'aise des élèves avec les concepts abordés.

\section{MATÉRIEL :}

Tableau noir + craie

\section{PRÉPARATION :}

Familiarisez-vous avec les cinq étapes de résolution de conflit en réfléchissant à la manière dont vous les appliqueriez à une situation qui vous concerne. Au besoin, adaptez l'exemple à une situation plus appropriée pour votre groupe. Veillez dans ce cas à choisir un exemple simple et réfléchissez à la présentation de chaque étape. Ces étapes peuvent paraître étranges aux élèves dans un premier temps mais leur application à de simples exemples les rendront plus familières. (Même les enfants plus jeunes peuvent aprendre à les suivre mais si vous trouvez l'activité trop intense, limitez-la aux étapes 2 et 4 ). 
[Reconnaissez que plusieurs réponses peuvent être valables et écrivez l'une de celles données pour compléter la deuxième invite.]

- Quelle est la différence entre dire « J'imagine (ou j'ai peur) que tu te fiches de mes sentiments » et «Tu te fiches de mes sentiments»?

4 Invitez un élève à lire les deux premières invites, $y$ compris les mots ajoutés, puis écrivez la troisième : «ET JE ME SENS ALORS » Discutez :

- Concentrez-vous sur l'émotion exacte que Ron ressent peut-être : pas sur ce qu'il pense, mais sur ce qu'il ressent.

- Quels sentiments peut-on avoir quand on pense qu'un ami se moque de nous avec d'autres? [Sondez: blessé, trahi, en colère, gêné, seul, humilié.]

[Complétez la troisième invite en écrivant au tableau deux ou trois des mots suggérés pour décrire ces sentiments.]

5 Invitez un élève à lire le message complet écrit au tableau jusqu'ici, puis écrivez la quatrième invite : «ET ÇA ME DONNE ENVIE DE ». Discutez :

- Que pourrait vouloir faire Ron?

[Sondez : Ça me donne envie de... casser du sucre sur ton dos aussi ; hurler; ne plus être ton ami; ou

m'enfermer chez moi et ne plus voir personne.] [Complétez la quatrième invite en écrivant au tableau une ou deux réponses données. Rappelez aux élèves que ce qu'on peut avoir envie de faire à un moment donné est souvent différent de ce qu'on va faire.]

6 Invitez un élève à lire le message complet, puis écrivez la dernière invite : «ET POURTANT JE ". Discutez:

- Dans certaines situations, une personne peut se sentir si violée qu'elle ne désire plus avoir le moindre contact avec l'autre. Souvent pourtant, elle désire malgré tout maintenir la relation.

Comment l'autre (Kim, dans notre exemple) peutil se sentir après avoir entendu ceci ?

- Comment Ron peut-il offrir l'assurance simple et directe de ce qu'il ressent globalement?

[Sondez: «Et pourtant, je...

t'aime toujours bien»; ou

«je veux rester ton ami $»$.

[Au tableau, écrivez une réponse appropriée à la dernière invite.]

7 Lisez le message au complet. Invitez les commentaires. 
8 Organisez les élèves deux par deux et expliquez:

- À votre tour de pratiquer ces étapes. Pensez à un conflit que vous ne croyez pas avoir résolu au mieux. [Ā défaut d'exemple personnel, proposez l'une des situations suivantes:

Votre père est très malade et votre meilleur(e) ami(e) ne s'y intéresse pas vraiment.

Votre cousin dit à vos amis la vérité sur votre père, qui est en prison.

Votre amie ne partage jamais son livre avec vous. Elle sait pourtant que vous avez perdu le vôtre et que vous ne pouvez pas le remplacer.

Votre ami est toujours en retard quand vous devez faire quelque chose ensemble.

Votre petite amie parle toute la soirée à un autre garçon.

Votre petit ami dit que si vous l'aimiez vraiment, vous auriez des rapports sexuels avec lui.

Vos amis vous poussent à perdre votre virginité.

Votre petite amie s'énerve quand vous voulez utiliser le préservatif.]

- L'un des partenaires dit à l'autre le conflit dont il ou elle va parler.

- Suivez les cinq invites. Vous avez environ 5 minutes pour couvrir, une à la fois, toutes les étapes. Ne vous en faites pas si cela vous paraît étrange au début. C'est la fin qui compte.
- Le partenaire qui écoute peut aider l'autre à se souvenir de notre discussion de chaque étape.

9 Passez une fois encore les cinq étapes en revue avec les élèves :

Quand tu ___ (comportement spécifique) j'imagine (ou commence à penser) que et je me sens alors ___ (un mot décrivant une émotion)

et ça me donne envie de et pourtant je (te)

10 Donnez 5 minutes de pratique à chaque partenaire.

11 Encouragez la discussion :

- Avez-vous pu vous exprimer différemment cette fois-ci, par rapport à la première fois où vous avez peut-être essayé de gérer ce conflit?

- Ces mots vous aident-ils à exprimer vos véritables sentiments?

- Vous sentez-vous mieux à l'idée de mieux identifier et exprimer vos sentiments?

- N'oubliez pas que vous avez le droit d'être traités avec respect. Si vous ne vous sentez pas respectés, vous avez le droit de vous défendre. Qui le fera sinon? 


\section{parler sexe et santé sexuelle}

\section{DURÉE :}

Étapes 1-4: 40 minutes

Étapes 5-6: 30 minutes

\section{MATÉRIEL :}

Tableau noir + craie ; ruban adhésif et marqueurs; carton pour affiches ou grandes feuilles de papier ;

descriptions des sketchs et affiches.

\section{PRÉPARATION :}

Passez en revue les descriptions de sketchs et d'affiches et adaptez-les à votre contexte. Si vous pensez diviser cette activité en deux sessions, décidez où arrêter la première. Copiez chaque description de sketch et d'affiche sur une feuille différente à distribuer. Réfléchissez à la manière dont vous allez intensifier la présentation et interrompre la première interprétation (en battant par exemple des mains pour lancer la deuxième).

APERC̣U: Par discussion et jeu de rôle, les élèves pratiquent les aptitudes verbales et non verbales nécessaires à la communication sur les décisions, la santé et les comportements sexuels.

OBJECTIFS : Renforcer les aptitudes verbales et non verbales nécessaires à la communication sur les décisions et la santé sexuelles; renforcer l'esprit d'analyse et l'aptitude au dialogue.

\section{INSTRUCTIONS}

1 Présentez l'activité en demandant:

- Les jeunes parlent peut-être de sexe entre amis et les médias y font référence, mais est-il facile, pour la plupart, d'en parler avec leur propre petit ami ou petite amie?

- Est-il facile ou non de répondre à une personne qui vous fait des avances sexuelles?

- Pourquoi est-il important de savoir communiquer avec cette personne sur les questions de comportement et de santé sexuels? [Sondez : pour établir clairement si vous voulez un contact sexuel ou non; avec quel type de contact vous vous sentez à l'aise, et ce avec quoi l'autre se sent à l'aise; pour vous protéger contre les IST (y compris le VIH); pour clarifier les intentions relatives à la grossesse et pour éviter une grossesse non désirée.]
- Nous allons nous organiser en couples et en petits groupes. Huit élèves (quatre couples) vont préparer un sketch d'une minute sur une question particulière, pour illustrer la communication assurée. Le reste des élèves vont former quatre groupes chargés de créer des affiches présentant chacune trois façons de lancer une conversation difficile particulière.

2 Organisez les couples et les groupes et distribuez les descriptions de sketch et d'affiche.

3 Invitez chaque couple à interpréter son sketch d'une minute. Pour chacun, dès que la personne A cède, dites « STOP! DE L'ASSURANCE! » pour lancer la deuxième version (assurée) du sketch. 
4 Après les quatre sketchs, encouragez la discussion. Demandez :

- Quelle version (passive ou assurée) vous paraît la plus courante?

- Qu'avez-vous appris d'utile?

- Quelles autres suggestions auriez-vous pour gérer ce type de conversation?

- Où peut-on espérer que ce dialogue conduira?

5 Invitez chaque groupe à présenter son affiche. Demandez à une personne du groupe de présenter l'affiche et à une autre de répondre aux questions. Invitez les commentaires et les suggestions. (Peutêtre voudrez-vous garder les affiches au mur pendant quelques jours.)

6 Pour conclure, posez les questions suivantes:

- Nous avons vu qu'il peut être plus difficile de se défendre dans une situation sexuelle quand l'autre personne occupe un rang plus élevé ou a plus de pouvoir. N'oubliez pas que vous avez toujours le droit de consentir à une situation sexuelle ou de la refuser.

\section{- Comment peut-on se préparer à entamer une conversation difficile ayant trait à la sexualité ? [Sondez: en répétant devant un miroir; en écrivant à l'avance ce qu'on veut dire.]}

Devoir : Pensez à une conversation (à propos d'un sujet quelconque) qu'il vous est difficile d'entamer. Rédigez la manière dont vous pourriez commencer. N'oubliez pas que quelqu'un pourrait pensez que vous avez eu cette conversation. Pour éviter toute violation de votre confidentialité, veillez donc à détruire votre script après l'avoir écrit. Prenez cependant le temps de l'écrire : vous vous sentirez probablement mieux. 


\section{sketchs}

\section{COUPLE 1 (GARCON/FILLE):}

Présentez une conversation d'une minute sur la prévention d'une infection sexuellement transmissible. A aborde le sujet. $B$, plus âgé(e), ne veut pas coopérer. Attribuez les rôles comme bon vous semble. Vous allez interpréter la scène deux fois. La première fois, A cède et accepte de ne pas utiliser le préservatif. Après interruption par l'enseignant, recommencez la scène en montrant cette fois comment A peut insister et poursuivre la conversation.

\section{COUPLE 2 (GARCQON/FILLE):}

A entame la conversation sur la prévention de la grossesse. B désire un enfant et résiste à l'idée de la contraception. Attribuez les rôles comme bon vous semble. Vous allez interpréter la scène deux fois. Pour la première, A cède et accepte de ne pas pratiquer la contraception. Après interruption par l'enseignant, recommencez la scène en montrant cette fois comment A peut insister et poursuivre la conversation.

\section{COUPLE 3 (GARÇON/FILLE):}

A et $\mathrm{B}$ vont à l'école ensemble. Attribuez les rôles comme bon vous semble. A met constamment son bras autour de la taille de B sur le chemin de l'école. Cela ne plaît pas à B. Vous allez interpréter la scène deux fois. Pour la première, $B$ n'apprécie pas mais ne dit rien. Après interruption par l'enseignant, recommencez la scène, en montrant cette fois comment $\mathrm{B}$ peut réagir de manière assurée.

\section{COUPLE 4 (GARC̣ON/FILLE) :}

A (un homme adulte occupant une position de leader au centre communautaire) met son bras autour des épaules d'une fille, $B$, au moment où elle arrive au centre. Vous allez interpréter la scène deux fois. La première fois, $B$ n'apprécie pas mais ne réagit pas. Après interruption par l'enseignant, recommencez en montrant cette fois comment $B$ peut réagir de manière assurée. 


\section{affiches}

\section{GROUPE 1:}

Parlez de la manière de dire à un(e) partenaire intime que vous avez peut-être une infection sexuellement

transmissible. Créez une affiche présentant trois façons d'entamer la conversation. Vous pouvez aussi illustrer l'affiche.

\section{GROUPE 2 :}

Parlez de la manière de dire à votre petit(e) ami(e) que vous désirez avoir des rapports sexuels et de découvrir s'il ou elle partage votre désir. Créez une affiche présentant trois façons d'entamer la conversation. Vous pouvez aussi illustrer l'affiche de deux personnes en conversation sur cette question.

\section{GROUPE 3 :}

Parlez de la manière dont une personne peut entamer la conversation pour révéler des sévices sexuels à un parent ou à une personne de confiance. Créez une affiche présentant trois façons d'entamer la conversation. Vous pouvez aussi illustrer l'affiche de deux personnes en conversation sur cette question.

\section{GROUPE 4 :}

Parlez de la manière dont une personne qui a eu des rapports sexuels peut dire à son ou sa partenaire qu’elle désire poursuivre la relation mais que les rapports sexuels la mettent mal à l'aise. Créez une affiche présentant trois façons d'entamer la conversation. Vous pouvez aussi illustrer l'affiche de deux personnes en conversation sur cette question. 


\section{exécuter une décision difficile}

activité

39

\section{DURÉE :}

Étapes 1-5: 40 minutes

Étape $6: 80$ minutes (en classe ou comme devoir)

\section{MATÉRIEL :}

Tableau noir + craie ; 2 feuilles à distribuer aux élèves ; papier +

fournitures disponibles (crayons de couleur, marqueurs et stylos).

\section{PRÉPARATION :}

Dans le volume GUIDE (compagnon de ce volume), lisez la section de l'unité 5 consacrée à l'exécution des décisions, ou référez-vous à votre propre programme. Lisez la feuille « exemples de décisions difficiles » et sélectionnez celles que vous voulez utiliser, ou imaginez-en d'autres adaptées à votre contexte. Si possible, photocopiez « 8 étapes d'une décision difficile » et « exemples de décisions difficiles » pour chaque élève (vous pouvez combiner les deux en une seule feuille à distribuer)
APERCU : Les élèves apprennent les étapes fondamentales de mise en œuvre d'une décision et créent une bande dessinée présentant l'exécution d'une décision difficile.

OBJECTIFS : Renforcer la capacité des élèves à exécuter judicieusement leurs décisions difficiles ; renforcer la réflexion analytique et l'aptitude au dialogue.

\section{INSTRUCTIONS}

1 Pour présenter le sujet, demandez:

- Pensez à une situation où vous avez pris une décision mais avez eu du mal à l'exécuter. [Par exemple : faire vos devoirs dès que vous rentrez chez vous; utiliser le préservatif; rompre une relation malheureuse; inviter une fille ou un garçon à sortir; parler à un parent d'une question sensible; dire à un ami qu'il vous a vexé; ou vous soumettre à un test de dépistage du VIH.]

- Écrivez l'objet de la décision puis réfléchissez à tous les facteurs qui ont rendu son exécution difficile. Écrivez-les aussi.

- Quels peuvent être les obstacles à l'exécution d'une décision?

2 Distribuez la feuille « 8 étapes ... » et dites au groupe (en prenant un autre exemple que celui de la bourse s'il le faut) :

- Imaginons donc la prise d'une décision.

- Disons que vous avez décidé de faire une demande de bourse pour aller à l'université dans un autre pays et réaliser ainsi votre rêve.
3 Utilisez cet exemple (ou l'autre de votre choix) pour guider les élèves à travers les huit étapes :

ÉTAPE 1 : Qu'est-ce qui vous aiderait à exécuter votre décision? [Sondez : identifier les bourses disponibles; obtenir de bonnes notes; vérifier les conditions de la bourse; avoir accès à un ordinateur ou à une machine à écrire pour préparer votre demande.]

ÉTAPE 2: Quels seront les obstacles les plus probables? [Sondez: pas d'accès aux options possibles; opposition parentale; problème de langue; pas le temps de préparer le dossier nécessaire; peur de demander une lettre de recommandation; notes trop faibles; frais de transport élevés.]

ÉTAPE 3 : Comment « répéter » votre décision en privé et sans risques? [Sondez: préparer une demande écrite à un enseignant; s'exercer à aborder ses parents; lire les instructions relatives à la bourse et réfléchir aux raisons qui font de vous un bon candidat.] 
ÉTAPE 4 : Discutez la décision et le plan avec une personne dont vous avez l'appui. Qui ? [Sondez : un ami, un enseignant, un parent, un représentant $d u$ bureau des bourses; une personne qui valorise l'éducation universitaire; quelqu'un qui a déjà obtenu une bourse.]

ÉTAPE 5 : Réfléchissez au moyen d'exécuter votre décision sans risques. Quels sont les risques potentiels? [Sondez pour cet exemple : risque de grave conflit avec un parent; conséquences graves de toute approche malhonnête; risques potentiels d'un séjour dans un autre pays (si la bourse est obtenue).]

ÉTAPE 6 : Exécutez votre décision selon votre plan. Que feriez-vous effectivement? [Sondez : parler à un ami qui vous soutient; bien préparer votre approche à l'enseignant; réfléchir au meilleur moyen d'aborder un parent peut-être récalcitrant; travailler pour avoir de bonnes notes, puis préparer la demande.]
ÉTAPE 7 : En cas d'obstacles, préparez un autre plan d'action ou repensez votre décision. Par exemple, si vos parents ne sont pas à l'aise à l'idée que vous étudiiez à l'étranger, que pourriez-vous faire d'autre? [Demander une bourse dans votre pays.] Et si vos notes ne sont pas suffisantes pour cette bourse particulière? [En rechercher une autre.]

ÉTAPE 8 : N'oubliez pas que même en l'absence d'obstacle, vous avez le droit de réévaluer vos décisions et de changer d'avis. Quels facteurs pourraient vous amener à reconsidérer votre décision de demander une bourse à ce stade, même en l'absence d'obstacles?

[Sondez : l'université locale vous propose le même programme; importance de rester proche de votre famille; peur soudaine de vous éloigner; besoin de travailler à mi-temps pendant vos études; décision de différer vos projets d'un an.]

[Peut-être voudrez-vous souligner ici la valeur de l'éducation en disant, par exemple: "Si l'un d'entre vous a jamais l'occasion de faire des études supérieures, j’espère que vous la saisirez. »] 
4 Distribuez la feuille d'exemples de décisions difficiles et expliquez:

- Pour pratiquer ces étapes, vous allez chacun et chacune choisir une décision dans cette liste.

Votre tâche est de créer une bande dessinée basée sur un personnage qui a pris cette décision. Montrez l'évolution de ce personnage à travers notre modèle de décision en huit étapes. Vous pouvez couvrir certaines étapes rapidement : votre personnage peut par exemple indiquer simplement ce qu'il ou elle fait. Veillez cependant, pour au moins une ou deux étapes, à entrer dans le détail.

- Vous pouvez commencer votre bande dessinée pendant le temps qui nous reste. Passez ensuite encore une heure au moins à l'achever.

[Remarque: Vous pouvez donner la seconde heure comme devoir, ou prévoir une seconde session.

Prenez le temps de vérifier que les élèves ont bien compris la tâche.]
5 Invitez les élèves à se mettre au travail. Parcourez la salle pour vous assurer qu'ils comprennent tous la tâche et pour les aider à bien appliquer le modèle en huit étapes.

6 (Peut être donné comme devoir): Donnez plus de temps aux élèves pour achever la tâche. Laissezles échanger quelques idées les uns avec les autres. Considérez d'afficher les projets terminés ou d'en faire un album. 


\section{8 étapes d'une décision difficile}

ÉTAPE 1 : Considérer les facteurs qui faciliteront l'exécution.

ÉTAPE 2: Réfléchir aux obstacles probables.

ÉTAPE 3: Répéter l'exécution de la décision en privé ou sans risques.

ÉTAPE 4: Discuter la décision et le plan avec une personne dont on a l'appui.

ÉTAPE 5: Trouver le moyen le moins risqué d'exécuter sa décision.

ÉTAPE 6: Exécuter la décision selon le plan élaboré.

ÉTAPE 7: En cas d'obstacles, élaborer un autre plan d'action ou repenser la décision.

ÉTAPE 8: Ne pas oublier que même en l'absence d'obstacles, on a toujours le droit de réévaluer ses décisions et de changer d'avis. 


\section{exemples de décisions difficiles}

Vous avez décidé d'essayer d'obtenir une bourse pour aller étudier à l'université dans un autre pays.

Vous avez décidé de vous opposer à épouser la personne que vos parents ont choisie pour vous.

Vous avez décidé d'épouser quelqu'un que vos parents n'approuvent pas.

Vous avez décidé d'utiliser le préservatif chaque fois que vous avez des rapports sexuels.

Vous avez décidé de ne pas avoir de rapports sexuels avec votre partenaire même s'il (si elle) menace de vous quitter.

Vous avez décidé de rompre avec votre partenaire intime (fille ou garçon).

Vous avez décidé de dire à vos parents que vous (ou votre partenaire) êtes enceinte.

Vous avez décidé d'arrêter de fumer (ou de boire).

Vous avez décidé de quitter un partenaire violent.

Vous avez décidé de dire à vos parents que vous êtes homosexuel(le).

Vous avez décidé de vous soumettre à un test de dépistage du VIH.

Vous avez décidé de dire à votre partenaire que vous n'éprouvez pas de plaisir lors de vos rapports sexuels.

Vous avez décidé de dire à votre conjoint, partenaire sexuel(le) ou à vos parents que vous êtes séropositif(ve) à VIH.

Vous avez décidé de ne pas adopter une pratique courante dans votre communauté (par exemple, la mutilation génitale des filles, l'abandon de l'école à un certain âge ou l'adhésion à un gang).

Vous avez décidé de dire à votre partenaire ou conjoint que vous savez qu'il ou elle vous trompe.

Vous avez décidé de dire à votre partenaire ou conjoint que vous lui avez été infidèle. 


\section{ce que vous voyez en moi}

APERÇU : Les élèves parlent des pressions relatives à l'apparence physique. Par écrit, ils décrivent un aspect de leur apparence qui leur plaît. En petits groupes, chaque élève découvre les traits non liés à l'apparence que les autres admirent en lui ou en elle.

OBJECTIFS: Aider les élèves à décrire l'effet sur l'estime de soi des pressions culturelles relatives à l'apparence; les sensibiliser davantage à leurs propres qualités (liées ou non à leur apparence); renforcer leur sensibilité envers leurs pairs.

\section{INSTRUCTIONS}

1 Ouvrez la discussion en posant les questions-guides suivantes :

- De nombreux facteurs influencent la manière dont nous percevons notre corps. Est-il fort et en bonne santé ? La société au sens large et les médias présentent-ils comme attrayants tous les types de corps? Comment se sent-on quand on doit s'habituer à une foule de changements corporels en peu de temps ? La société met-elle trop l'accent sur l'apparence et pas assez sur nos autres qualités? Nous allons explorer certaines de ces questions aujourd'hui.

- Souvenez-vous du temps où vous aviez neuf ans. Que pensent la plupart des enfants de cet âge de leur corps et de leur apparence? S'en soucientils beaucoup ? [Invitez les élèves à exprimer leurs réponses sur une échelle de 1 à 10 , où $1=i l s$ ne s'en soucient pas du tout et $10=i l$ s'en soucient énormément.]
- Que se passe-t-il à l'adolescence ? La plupart des adolescents sont-ils insouciants et à l'aise vis-àvis de leur apparence ou s'en inquiètent-ils? [Ici encore, les réponses peuvent être exprimées sur une échelle de 1 à 10.]

- Quels types de messages et d'images les adolescents reçoivent-ils du cinéma et de la publicité concernant leur apparence et les qualités physiques désirables? La pression est-elle plus intense sur les filles ou sur les garçons?

- Les adolescents sont-ils souvent jugés par leur apparence ? Désire-t-on généralement être jugé sur cette base? Quelles autres qualités aime-ton voir appréciées? [Demandez aux filles comme aux garçons. Sondez : intelligence, honnêteté, sens de l'humour, sens du travail, courage, gentillesse, qualités artistiques, musicales et sportives, générosité, équité, écoute, loyauté et autres traits de caractère. Écrivez au moins huit à dix de ces qualités au tableau. Soulignez que les filles aussi bien que les garçons veulent être appréciés pour ces qualités.]

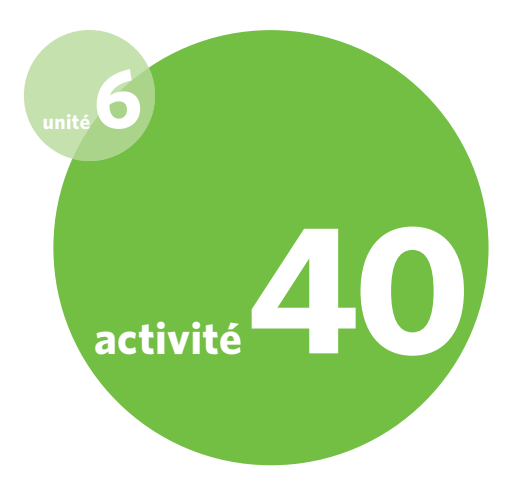

DURÉE :

45 minutes

\section{MATÉRIEL :}

Tableau noir + craie ; une feuille de papier vierge par élève (si possible, utilisez du papier de couleur, cartonné pour affiches ou autre papier spécial).

\section{PRÉPARATION :}

Si vous savez qu'il existe une relation hostile entre certains élèves, formez les petits groupes (étape 3) de manière à éviter qu'ils ne soient ensemble. II est crucial de minuter strictement l'activité, y compris à l'étape 3, pour que chaque élève puisse recevoir les compliments des autres. Si vous désirez explorer davantage l'influence de la publicité sur l'image corporelle, reportez-vous aussi à l'activité 9. 
2 Cahiers et stylos prêts, dites aux élèves :

- Pensez à un aspect de votre propre apparence ou de votre corps dont vous êtes satisfaits : votre sourire, vos yeux, votre démarche, vos muscles, vos cheveux ou votre taille. Ou bien la forme de votre corps, votre nez, votre façon de danser, la forme de votre visage, vos bras ou vos jambes, vos mains, vos fossettes ou vos lèvres. Ou bien quelque chose d'autre encore.

- Pour vous-mêmes - vous n'aurez pas à partager ceci- écrivez, sous forme poétique, une phrase décrivant cette caractéristique. Par exemple : "Mon sourire illumine toute la pièce » ou bien : «Mes yeux sont aussi profonds que l'océan ».

- Vous avez 10 minutes. Quand vous aurez terminé, mettez votre écrit à l'abri dans un endroit privé.

3 Cela fait, répartissez les élèves en groupes de cinq. Distribuez cinq feuilles vierges et un marqueur à chaque groupe. Expliquez:

- Nous avons vu que nous voulons tous être appréciés pour nos qualités autres que notre apparence.

- En commençant par un membre de votre groupe, l'un d'entre vous va écrire le nom de cette personne sur une feuille blanche. L'un après l'autre, vous allez dire à la personne nommée une qualité non liée à son apparence physique que vous appréciez en elle. Il peut s'agir d'une qualité mentionnée plus tôt [référez-vous au tableau] ou d'une autre caractéristique positive. Cette qualité peut vous être immédiatement évidente, ou peut-être voudrez-vous considérer un trait de la personne auquel vous n'aviez pas fait particulièrement attention jusqu'ici.

- Nommez cette qualité et écrivez-la sur la feuille revêtue du nom de la personne.

- Après avoir écrit votre mot, passez la feuille à un autre membre du groupe. Continuez jusqu'à ce que la feuille ait fait le tour du groupe.

- Répétez ensuite la démarche pour chaque membre restant du groupe. Veillez à exprimer votre compliment verbalement et par écrit. Prenez quelques minutes pour chaque personne. Je vous aiderai à gérer votre temps pour que chacun ait son tour. [Veillez à diviser le temps restant en cinq segments égaux. Circulez pour assurer la bonne progression de l'activité, dans le respect des autres.]

- Avant de commencer, rappelons les règles à observer pour cette activité? [Sondez : faire preuve de respect ; réfléchir à de nouveaux commentaires plutôt que de répéter ceux des autres; ne pas passer son tour; même si on n'aime pas trop un membre du groupe, reconnaittre que tout le monde a de bonnes qualités; traiter les autres comme on désire l'être.]

- Gardez, chacun et chacune, votre «feuille de compliments » : vous l'avez méritée ! 


\section{changements corporels}

APERCU: Les élèves écrivent un livre ou une chanson sur les changements physiques de la puberté.

OBJECTIFS : Aider les élèves à décrire les principaux changements physiques de la puberté ; renforcer l'esprit d'équipe et les aptitudes à la présentation d'information.

\section{INSTRUCTIONS}

1 Expliquez que cette leçon parle des changements physiques de l'adolescence. Demandez :

- À quel âge est-il bon d'apprendre les effets de la puberté sur le corps : après l'avoir atteinte ou plus tôt ? La plupart des enfants savent-ils suffisamment à quoi s'attendre à la puberté ?

- Vous allez créer un matériel sur la puberté destiné aux jeunes qui l'atteindront dans un an ou deux. Vous allez travailler par groupes de trois filles ou trois garçons. Les groupes de filles vont présenter les changements de la puberté qui affectent le sexe féminin et les groupes de garçons, ceux qui affectent le sexe masculin.

- Présentez votre information comme bon vous semble : composez par exemple une chanson hip-hop, écrivez un livre pour enfants, créez une affiche ou imaginez la lettre « Cher Docteur » d'un jeune enfant et la réponse publiée dans un journal. Soyez créatifs! Faites preuve d'humour, rimez, illustrez, pourvu que votre information soit exacte et utile. [Si les dispositions nécessaires ont été prises, expliquez aux élèves qu'ils vont présenter leur travail à un jeune public.]
- Pour vous aider, je vais distribuer à chaque groupe une fiche d'information sur la puberté (des garçons et des filles).

2 Organisez les groupes de trois et distribuez les fiches. Mettez le matériel à la disposition des groupes et dites-leur qu'ils ont 25 minutes.

3 Si vous avez prévu la présentation à de plus jeunes élèves: Passez le reste du temps à circuler dans la classe et à parler à chaque groupe de son produit fini avant d'en approuver la présentation. Aidez les groupes à rectifier ou compléter toute information incorrecte ou manquante.

Si la présentation se fera devant la classe seulement: Invitez les groupes à présenter leur travail durant le temps restant et, au besoin, lors de la prochaine session. Engagez les élèves des autres groupes à compléter ou rectifier l'information manquante ou incorrecte.

4 À la fin de la session, distribuez le reste des fiches d'information pour que chaque élève en ait une sur la puberté féminine et sur la puberté masculine.

\section{DURÉE :}

60 minutes (une ou deux sessions)

\section{MATÉRIEL :}

Fournitures nécessaires à la réalisation d'un livre (papier marqueurs, carton pour affiches ou grandes feuilles de papier, fil et aiguilles ou agrafeuse). Fiches d'information fiables sur la

puberté des garçons et des filles, telles que celles proposées dans le GUIDE (volume compagnon de cet ouvrage), accessible sur $<$ www.unseulprogramme.org .

\section{PRÉPARATION :}

Lisez et familiarisez-vous avec les fiches d'information pour être prêt à compléter ou corriger le travail des élèves. Photocopiez vos fiches sur la puberté des garçons et celle des filles. Si possible, organisez la présentation du travail de vos élèves à d'autres préadolescents. 


\section{la puberté, c'est aussi}

DURÉE :

45 minutes

\section{MATÉRIEL :}

Tableau noir + craie

bandelettes de papier ; «Info et clé pour l'enseignant »).

\section{PRÉPARATION :}

Copiez chaque « changement » listé sur la feuille « changements associés à la puberté et à

l'adolescence »s sur une bandelette différente, pliez la bandelette et glissez-la dans une enveloppe. Lisez les changements listés à l'étape 3 et modifiez ou complétez la liste pour décrire la manière dont la vie des jeunes change à l'approche de l'adolescence dans votre contexte.
APERCQU : Les élèves analysent les changements sociaux qui affectent les garçons et les filles pendant l'adolescence. (Remarque : L'activité 41 ou une autre leçon sur les changements physiques de la puberté est nécessaire avant cette activité.)

OBJECTIFS: Aider les élèves à citer trois changements qui affectent les rôles de genre à l'adolescence et à évaluer de manière critique l'impact de ces changements sur les filles, les garçons et les relations de genre ; renforcer la réflexion critique.

\section{INSTRUCTIONS}

1 Présentez le sujet :

- Tous les jeunes traversent une période de changements à la puberté et à l'adolescence : les uns de nature physique, les autres dans la manière dont ils sont traités.

- Je vais parcourir la classe et demander à certains d'entre vous de prendre une bandelette de papier, de la lire à haute voix et de nous dire s'il s'agit d'un changement physique tel que le vivent tous les jeunes du monde ou d'un changement du traitement reçu de la société à la puberté et à l'adolescence. [Si vous n'avez pas préparé de bandelettes, lisez vous-même, au hasard, les changements listés dans les deux colonnes de la feuille Info Enseignant.]

- Pour le reste de l'activité, nous allons examiner en détails les changements qui affectent la manière dont les jeunes sont souvent traités à l'adolescence. Je ne parle plus ici des changements physiques qui affectent votre corps.
2 Écrivez CHANGEMENTS AFFECTANT LA MANIĖRE DONT LES GENS VOUS TRAITENT comme titre général au tableau. Sous ce titre, écrivez GARÇONS d'un côté et FILLES de l'autre. Expliquez:

- Qu'arrive-t-il aux jeunes quand ils atteignent l'adolescence ? Sont-ils traités différemment? Dites-moi si les changements dont nous allons parler s'appliquent aux filles ou aux garçons. S'ils concernent les deux, dites-moi s'ils affectent différemment les filles et les garçons (et comment).

3 Lisez chaque point de la liste proposée à la page suivante (après l'avoir lue/modifiée) et demandez aux élèves s'il concerne les garçons, les filles ou les deux. En cas de désaccord, arrêtez et discutez la question avant d'inscrire le changement sous Garçons, Filles ou les deux. 
Adolescence : changements affectant la manière dont les gens vous traitent

- Plus de liberté à fréquenter l'espace public (rues, parcs, centres communautaires ou commerciaux)

- Moins de liberté à fréquenter l'espace public

- Plus de responsabilités domestiques (tâches ménagères ou soins des enfants)

- Plus grande responsabilité de gagner de l'argent

- Plus de pression à se vêtir de manière à se couvrir ou découvrir davantage le corps

- Plus d'interaction sociale entre les garçons et les filles ou moins d'interaction sociale

- Rites de passage avec pratiques nuisibles (telles que la mutilation génitale des filles)

- Rites de passage sans pratiques nuisibles

- Pression sociale d'acquérir une expérience sexuelle

- Pression sociale de se préparer au mariage

- Meilleures occasions de leadership à l'école et dans la communauté

- Pression sociale de performance sportive

- Risque accru de devoir quitter l'école si la famille en décide ainsi

- Exposition au harcèlement sexuel

- Pression de faire partie d'un gang

- Pression de se conformer aux rôles de genre en prenant des risques dangereux

- Pression d'accorder ses faveurs sexuelles en échange de cadeaux, d'argent ou d'argent pour l'école
4 Divisez la classe en petits groupes de même sexe et demandez-leur de discuter comment les rôles de genre et la vie des jeunes changent à la puberté et à l'adolescence. Plus précisément :

- Les changements d'attentes sociales et qui affectent la vie des jeunes à la puberté sont-ils considérables ou diriez-vous qu'ils sont plutôt mineurs?

- Que remarquez-vous dans les changements qui affectent les garçons à la puberté ? Dans l'ensemble, ont-ils plus ou moins de liberté ? Ces expériences perdent-elles de l'importance après la puberté ou se perpétuent-elles à l'âge adulte ?

- Que remarquez-vous dans les changements qui affectent les filles à la puberté ? Dans l'ensemble, sont-elles plus ou moins libres? Ces expériences perdent-elles de l'importance après la puberté ou se perpétuent-elles à l'âge adulte?

5 Rassemblez la classe au complet et demandez:

- Les choses pourraient-elles être différentes? Pourrait-on vivre de manière plus juste ou plus équitable? Quelle est votre vision?

- Citez une façon dont la société pourrait favoriser pour les jeunes une meilleure expérience de la puberté et de l'adolescence.

Devoir : Écrivez deux lettres brèves : " Promesse à ma future fille » et " Promesse à mon futur fils », en vous inspirant de la discussion d'aujourd'hui. 


\section{changements associés à la puberté et à l'adolescence}

INSTRUCTIONS POUR L'ENSEIGNANT : Copiez chaque point ci-dessous sur une bandelette de papier différente. Pliez les bandelettes et placez-les dans une enveloppe. Au besoin, servezvous de cette page comme clé de réponses pour distinguer correctement les changements biologiques (au niveau du corps) de ceux de nature sociale (traitement de la personne en fonction du genre).

\section{CHANGEMENTS AFFECTANT LE CORPS}

Pilosité (poils)

Transpiration accrue

Développement des seins (chez les filles)

Éjaculations nocturnes (chez les garçons)

Mue (changement du timbre de la voix chez les garçons)

Accélération de la croissance générale ; besoin

d'alimentation accrue

Accroissement des sentiments sexuels

Menstruation (règles)/sécrétions muqueuses (chez les filles)

\section{CHANGEMENTS DE TRAITEMENT}

Nouvelles occasions de leadership à l'école et dans la communauté

Rites de passage à l'âge adulte

Changements de responsabilités

Nouvelles pressions relatives à l'activité sexuelle

Nouvelles pressions relatives au mariage

Nouvelles règles d'habillement

Nouvelles règles d'interaction sociale entre garçons et filles

Changement de degré de liberté 


\section{le saviez-vous?}

APERCUU: Une grille de mots croisés et un exercice aident les élèves à apprendre et réviser quelques faits intéressants sur le corps et la reproduction. (Remarque : Cette activité s'inscrit en complément d'une leçon ou lecture sur la reproduction; elle ne représente nullement une introduction complète à la question.)

OBJECTIFS : Élargir la connaissance des élèves sur la reproduction humaine ; éveiller leur intérêt pour la biologie de la reproduction.

\section{INSTRUCTIONS}

1 Distribuez la grille de mots croisés et expliquez:

- Nous avons appris les principes de base de la reproduction humaine. Pour les réviser, lisez chaque définition et remplissez la grille.

- Chaque réponse doit remplir le nombre correct de cases.

- Au croisement d'une réponse verticale et d'une réponse horizontale, la lettre doit être identique.

2 Au bout de 10-15 minutes, vérifiez les réponses en circulant dans la classe.

3 Distribuez la feuille de faits intéressants et expliquez:

- Nous allons maintenant découvrir d'autres faits intéressants sur le corps humain, pour la plupart liés à la reproduction.

- Je vous donne 5 minutes pour lire la liste. Entourez ensuite le fait qui vous paraît le plus intéressant.
- Demandez à deux de vos voisins et voisines s'ils ont entouré le même fait ou un fait différent.

- Combien d'entre vous ont entouré le même fait ? Et des faits différents? [levée de mains]

- Combien d'entre vous ont appris au moins une nouvelle chose?

4 Invitez les volontaires à révéler le fait qu'ils ont trouvé particulièrement intéressant. Complétez brièvement l'information relative à ce fait en vous référant aux Matériel pour l'enseignant. Si possible, questionnez les élèves pour qu'ils développent eux-mêmes leur information.

5 S'il vous reste du temps: Au fait numéro 15, invitez les élèves à comparer, deux par deux, l'envergure de leurs bras à leur taille. Expliquez qu'il ne s'agit pas d'un fait directement lié à la reproduction mais qui fait partie de la connaissance de son corps.

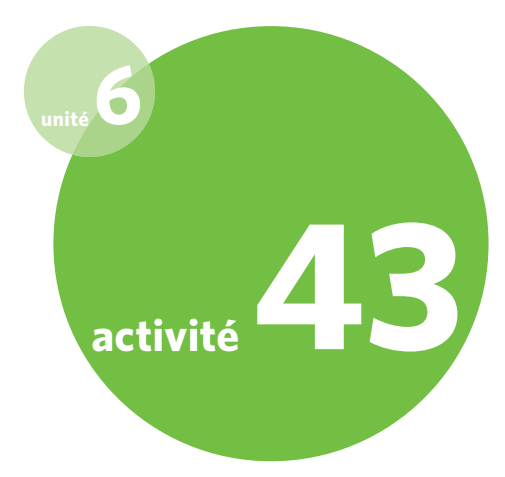

DURÉE :

Étapes 1-2: 20 minutes

Étapes $3-5: 25$ minutes

\section{MATÉRIEL :}

Une copie pour chaque élève de la grille de mots croisés, de la feuille de faits intéressants et source d'information fiable sur la biologie de la reproduction (telle que l'unité 6 et les fiches d'information du GUIDE compagnon de ce volume).

\section{PRÉPARATION :}

Lisez attentivement le matériel, concernant surtout le cycle menstruel, l'appareil sexuel et génital masculin et féminin, la reproduction et la grossesse, et la stérilité et la procréation assistée. Décidez de garder ou non les solutions aux mots croisés sur la feuille à distribuer ainsi que d'inclure ou non tous les faits intéressants suivant le temps disponible ou le contenu. 
mots croisés : I'appareil génital

Lisez chaque définition et remplissez la grille. Votre réponse doit correspondre au nombre de cases. Au croisement d'une réponse verticale et d'une réponse horizontale, la lettre doit être identique.

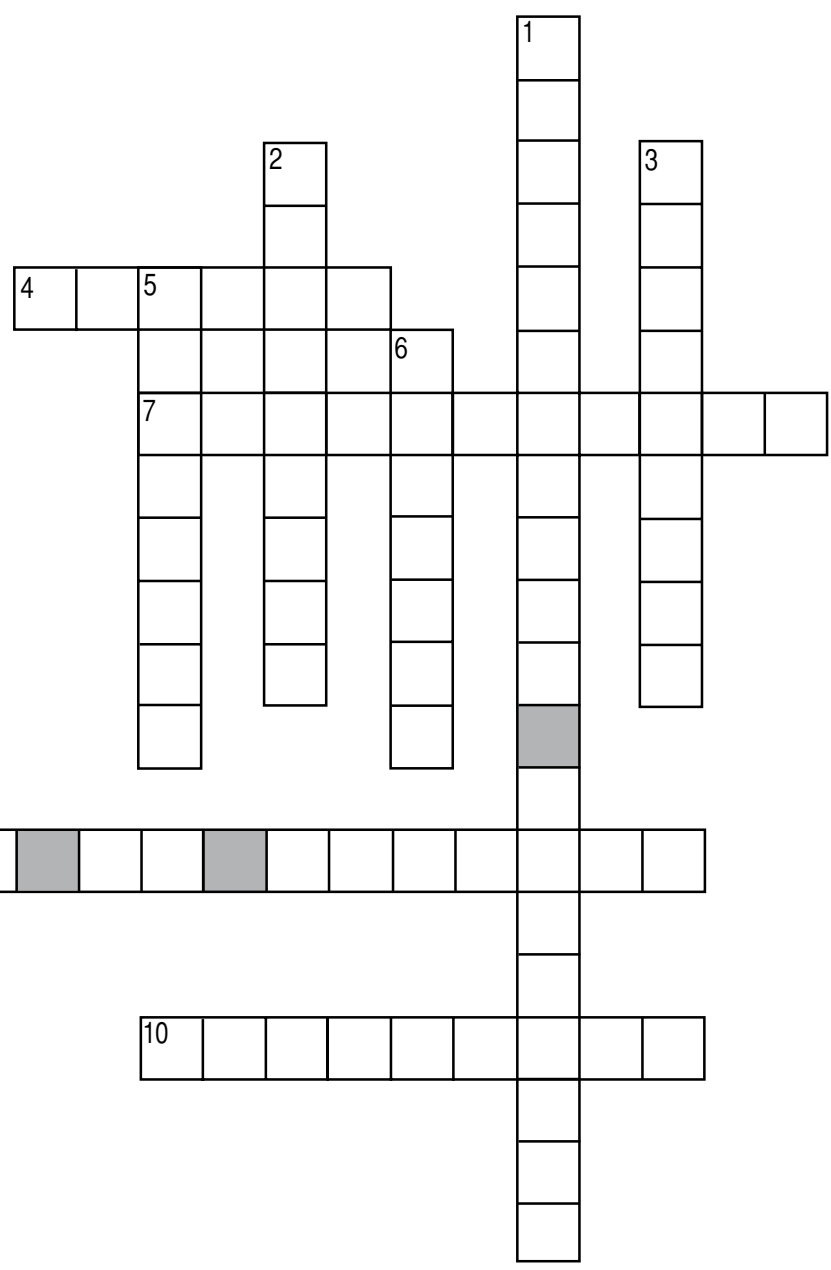

\section{à l'horizontale}

4. Endroit où un bébé se développe

7. Projection de sperme à travers le pénis

9. Tubes qui relient les ovaires à l'utérus :

la fécondation a lieu ici (3 mots)

10. Moment de la vie où la menstruation de la femme s'arrête à la verticale

1. Expulsion de sperme pendant le sommeil (2 mots)

2. Libération d'un ovule de l'ovaire

3. Lieu de production des spermatozoïdes

5. Durcissement du pénis

6. Période de développement rapide du corps de l'enfant vers le corps adulte et la capacité de se reproduire

8.Liquide épais émis par le pénis lors de l'éjaculation 


\section{faits intéressants sur le corps humain}

Lequel de ces faits vous paraît-il le plus étonnant et le plus intéressant ? Lisez la liste ci-dessous et entourez le fait qui vous paraît vraiment bon à savoir.

1 Beaucoup de femmes s'intéressent plus au sexe pendant les jours féconds de leur cycle menstruel.

2 Chez les couples qui ne réussissent pas à concevoir, la stérilité peut tout aussi bien venir de l'homme que de la femme.

3 L'intestin grêle humain mesure environ sept mètres.

4 Les spermatozoïdes se développent dans les testicules (à l'intérieur du scrotum). Leur bon développement nécessite un milieu plus frais que la température normale du corps. Situé à l'extérieur du corps, le scrotum assure ce milieu.

5 Si les femmes ne sont fécondes que quelques jours par cycle menstruel, les hommes le sont en permanence dès la puberté.

6 Un nombre normal de spermatozoïdes se mesure dans les dizaines de millions par éjaculation.

7 Le corps humain produit environ un litre de salive toutes les 24 heures.

8 Une femme ou une fille peut apprendre à reconnaître le moment où elle ovule par simple observation des changements de la glaire du vagin. Elle peut savoir ainsi le moment où elle est féconde (et peut tomber enceinte) et prédire le jour de ses prochaines règles.

9 Le corps humain est fait d'eau à plus de 50 pour cent.

10 La forme du ventre de la femme enceinte n'a rien à voir avec le sexe du bébé.

11 Vers la fin de la grossesse, les articulations des hanches de la femme se relâchent pour permettre au bassin de s'élargir et de faciliter ainsi le passage du bébé.

12 Dans de rares cas, la femme libère deux ovules plutôt qu'un. S'ils sont tous deux fécondés, de faux jumeaux (non identiques) se développent. Si un même ovule est fécondé et subit très tôt une division cellulaire supplémentaire, on a de vrais jumeaux.

13 La plupart des garçons ont des éjaculations nocturnes à la puberté.

14 Le sexe d'un fœtus est déterminé par le spermatozoïde du père, pas par l'ovule de la mère.

15 L'envergure de vos bras est égale à la longueur totale de votre corps. 


\section{« FAITS INTÉRESSANTS » - INFO COMPLÉMENTAIRE}

\begin{tabular}{|c|c|c|}
\hline & Fait intéressant & Info complémentaire \\
\hline 1 & $\begin{array}{l}\text { Beaucoup de femmes s'intéressent plus au sexe pendant les jours } \\
\text { féconds de leur cycle menstruel. }\end{array}$ & $\begin{array}{l}\text { Cet accroissement de la libido assure les rapports sexuels au moment le plus } \\
\text { propice à la perpétuation de l'espèce. (À discuter dans le contexte de l'évolution.) }\end{array}$ \\
\hline 2 & $\begin{array}{l}\text { Chez les couples qui ne réussissent pas à concevoir, la stérilité peut } \\
\text { tout aussi bien venir de l'homme que de la femme. }\end{array}$ & $\begin{array}{l}\text { Dans certains contextes, les femmes sont tenues responsables de la stérilité } \\
\text { parce que les hommes ignorent qu'ils peuvent en être la cause. Il est souvent } \\
\text { difficile de déterminer la cause exacte de la stérilité. (Voir aussi la fiche sur } \\
\text { la stérilité.) }\end{array}$ \\
\hline 3 & L'intestin grêle humain mesure environ sept mètres. & Dans le corps, l'intestin grêle s'enroule sur lui-même et prend peu d'espace. \\
\hline 4 & $\begin{array}{l}\text { Les spermatozoïdes se développent dans les testicules (à l'intérieur } \\
\text { du scrotum). Leur bon développement nécessite un milieu plus frais } \\
\text { que la température normale du corps. Situé à l'extérieur du corps, le } \\
\text { scrotum assure ce milieu. }\end{array}$ & $\begin{array}{l}\text { Une chaleur constante autour du scrotum (due au travail à proximité d'un } \\
\text { four ou au port de pantalons et de sous-vêtements serrés qui accroissent la } \\
\text { température du scrotum et des testicules, par exemple) peut réduire le nombre } \\
\text { de spermatozoïdes. (Voir aussi la fiche sur l'appareil sexuel et génital masculin.) }\end{array}$ \\
\hline 5 & $\begin{array}{l}\text { Si les femmes ne sont fécondes que quelques jours par cycle } \\
\text { menstruel, les hommes le sont en permanence dès la puberté. }\end{array}$ & $\begin{array}{l}\text { Avec l'âge, la fécondité diminue graduellement. (Voir aussi la fiche sur le cycle } \\
\text { menstruel). }\end{array}$ \\
\hline 6 & $\begin{array}{l}\text { Un nombre normal de spermatozoïdes se mesure dans les dizaines } \\
\text { de millions par éjaculation. }\end{array}$ & $\begin{array}{l}\text { Les spermatozoïdes arrivent à maturité dans les testicules en l'espace d'environ } \\
75 \text { jours. (Voir aussi la fiche sur l'appareil sexuel et génital ma\sculin.) }\end{array}$ \\
\hline 7 & $\begin{array}{l}\text { Le corps humain produit environ un litre de salive toutes les } 24 \\
\text { heures. }\end{array}$ & $\begin{array}{l}\text { À base d'eau, la salive contient aussi des substances chimiques, dont des } \\
\text { enzymes servant à décomposer la nourriture. }\end{array}$ \\
\hline 8 & $\begin{array}{l}\text { Une femme ou une fille peut apprendre à reconnaître le moment où } \\
\text { elle ovule par simple observation des changements de la glaire du } \\
\text { vagin. Elle peut savoir ainsi le moment où elle est féconde (et peut } \\
\text { tomber enceinte) et prédire le jour de ses prochaines règles. }\end{array}$ & $\begin{array}{l}\text { Si la femme sait quand elle ovule, elle peut prendre les mesures nécessaires pour } \\
\text { éviter une grossesse ou, au contraire, pour essayer de concevoir. (Voir aussi la } \\
\text { fiche sur le cycle menstruel.) }\end{array}$ \\
\hline
\end{tabular}




\begin{tabular}{|c|c|c|}
\hline & Fait intéressant & Info complémentaire \\
\hline 9 & Le corps humain est fait d'eau à plus de 50 pour cent. & Et votre organe le plus grand est votre peau! \\
\hline 10 & $\begin{array}{l}\text { La forme du ventre de la femme enceinte n'a rien à voir avec le sexe } \\
\text { du bébé. }\end{array}$ & $\begin{array}{l}\text { Une étude du ventre des femmes enceintes n'a établi aucun rapport entre la } \\
\text { forme du ventre et le sexe du bébé. }\end{array}$ \\
\hline 11 & $\begin{array}{l}\text { Vers la fin de la grossesse, les articulations des hanches de la } \\
\text { femme se relâchent pour permettre au bassin de s'élargir et de } \\
\text { faciliter ainsi le passage du bébé. }\end{array}$ & $\begin{array}{l}\text { La femme doit parfois faire attention à ne pas tomber vers la fin de sa grossesse } \\
\text { car ce relâchement des articulations peut la faire vaciller. (Voir aussi la fiche sur } \\
\text { l'accouchement et l'allaitement maternel.) }\end{array}$ \\
\hline 12 & $\begin{array}{l}\text { Dans de rares cas, la femme libère deux ovules plutôt qu'un. S'ils } \\
\text { sont tous deux fécondés, de faux jumeaux (non identiques) se } \\
\text { développent. Si un même ovule est fécondé et subit très tôt une } \\
\text { division cellulaire supplémentaire, on a de vrais jumeaux. }\end{array}$ & $\begin{array}{l}\text { Les faux jumeaux présentent les gènes de frères et sœurs ordinaires, tandis } \\
\text { que les vrais jumeaux viennent d'un même noyau. Leur ADN (leurs gènes) est } \\
\text { pratiquement identique. (Voir aussi la fiche sur la reproduction et la grossesse.) }\end{array}$ \\
\hline 13 & La plupart des garçons ont des éjaculations nocturnes à la puberté. & $\begin{array}{l}\text { Une éjaculation nocturne se produit quand un garçon ou un homme éjacule } \\
\text { dans son sommeil. Ces éjaculations sont parfaitement normales et pas du tout } \\
\text { néfastes. }\end{array}$ \\
\hline 14 & $\begin{array}{l}\text { Le sexe d'un fotus est déterminé par le spermatozoïde du père, pas } \\
\text { par l'ovule de la mère. }\end{array}$ & $\begin{array}{l}\text { Dans certains contextes, la culture fait pression sur les femmes pour qu'elles } \\
\text { produisent des garçons. C'est pourtant le spermatozoïde du père qui détermine } \\
\text { le sexe de l'enfant. L'embryon reçoit en effet un chromosome X de l'ovule de } \\
\text { la mère et un chromosome } X \text { ou } Y \text { du spermatozoïde du père : si X, l'embryon } \\
X X \text { est féminin et si } Y \text {, l'embryon XY est masculin. (Voir aussi la fiche sur la } \\
\text { reproduction et la grossesse). }\end{array}$ \\
\hline 15 & L'envergure de vos bras est égale à la longueur totale de votre corps. & Vérifiez! \\
\hline
\end{tabular}




\section{la procréation : pas toujours une affaire privée}

APERCU : Les élèves découvrent une quinzaine de questions de santé publique et de politique liées à la procréation (de la paternité adolescente à la sélection du sexe), y réagissent personnellement et les résument à des fins d'éducation publique.

OBJECTIFS: Informer et sensibiliser les élèves aux aspects politiques et de santé publique de la procréation; renforcer l'expression écrite et les aptitudes à l'édition ; renforcer leur conviction qu'ils ont une voix dans la communauté.

\section{DUREE :}

Étapes 1-6:45 minutes

Étape 7 (devoir) : 40 minutes

Étapes 8-11 (facultatif) : 40 minutes

\section{MATÉRIEL :}

Copies des feuilles à distribuer. Pour les élèves appelés à couvrir les sujets A à F, copies des fiches d'information sur la sélection du sexe, la stérilité et la procréation assistée, l'accouchement et l'allaitement maternel et l'avortement (IVG) du GUIDE compagnon de ce volume accessibles aussi sur < $w w w$. unseulprogramme.org>).

\section{PRÉPARATION :}

Lisez et adaptez (au besoin) les sujets à traiter. Photocopiez les feuilles à distribuer ou écrivez la liste finale des sujets au tableau avant la classe. Photocopiez les fiches sélectionnées. Explorez où les élèves pourraient soumettre une compilation de leur travail (à un journal local, sous forme de série de « commentaires-minute » à la radio, lors d'une assemblée à l'école ou sous forme de livre).

\section{INSTRUCTIONS}

1 Expliquez:

- Nous avons étudié la reproduction humaine. S'agit-il toujours d'une affaire d'ordre privé, intéressant les seuls individus directement concernés ? Ou bien certains aspects de la procréation présentent-ils un intérêt public, au niveau des autorités sanitaires ou de la communauté, par exemple? [Sondez : financement des services de santé, lois sur l'avortement, règlements des hôpitaux, notamment.]

- En fait, la santé génésique est une importante question d'ordre public, souvent controversée. Dans le monde moderne, face aux nouvelles technologies, au VIH et aux normes culturelles changeantes, il importe d'en être informé et de savoir comment elle affecte la vie.

- Nous allons commencer aujourd'hui un projet où vous allez explorer un sujet qui vous intéresse ou qui revêt pour vous un sens personnel. Le produit fini de votre travail sera présenté à la communauté.
[Expliquez comment : à travers un article publié dans un journal local, par exemple.]

2 Lisez la liste de sujets, en invitant les élèves à offrir de brèves définitions ou explications pour que tous se fassent pour le moins une idée générale de la question, puis expliquez:

- Vous allez choisir, chacun et chacune, un sujet qui vous intéresse ou qui revêt pour vous une importance ou un sens personnel. Il ne s'agit pas d'un long projet de recherche : vous allez rédiger, au maximum, deux longs paragraphes, en vous aidant de questions-guides. Mais vous allez rédiger ces deux paragraphes dans votre meilleur style.

- Vous allez aujourd'hui choisir votre sujet et vous informer un peu sur la question. Pour certains sujets, je vous donnerai un paragraphe ou une page à lire. Pour d'autres, vous allez interviewer trois de vos camarades de classe et prendre note de leurs points de vue. 
3 Passez en revue les instructions relatives au devoir. Si vous ne disposez pas de copies à distribuer aux élèves, copiez les instructions relatives au « flash d'information » au tableau.

\section{4 [Pour l'activité facultative d'édition:]}

Demain, vous échangerez votre deuxième paragraphe seulement (un article de journal) avec un(e) camarade et vous relirez ensemble votre travail pour vérifier qu'il est clair, grammaticalement correct, bien organisé et intéressant. Nous compilerons tous les textes en un article de « Réflexions sur la grossesse et la naissance » à soumettre à [un journal local, par exemple, ou une station de radio].

5 Vérifiez que la tâche est bien comprise. Faites circuler la feuille de sélection des sujets. Encouragez les élèves à vous demander de clarifier un sujet.

Invitez-les à choisir un sujet que personne d'autre n'a encore sélectionné.
6 Pendant le temps restant :

Pour les sujets A à F : Distribuez les copies du matériel à lire et invitez les élèves à commencer leur lecture. (Si vous disposez de copies qu'ils peuvent emporter chez eux, ils pourront achever leur lecture comme devoir.)

Pour les sujets $G$ à $N$ : Invitez les élèves à commencer à s'interviewer les uns les autres et à prendre des notes. Encouragez-les à rechercher différentes opinions. (Les interviews peuvent aussi être effectués à domicile, auprès de membres de la famille.)

7 Réservez 5 minutes à l'explication du devoir.

\section{Devoir:}

Si vous n'avez pas terminé votre lecture, finissez-la chez vous. Vous pouvez aussi achever vos interviews chez vous. Rédigez ensuite vos deux paragraphes. N’oubliez pas de répondre aux questions posées et d'écrire chaque paragraphe sur une feuille séparée.

Voir les étapes 8 à 11 (activité d'édition facultative) à la page suivante. 


\section{ACTIVITÉ FACULTATIVE :}

\section{RÉVISION, ÉCHANGE ET PRODUCTION}

8 Invitez les élèves à s'organiser deux par deux pour la révision de leur deuxième paragraphe (flash d'information). Si plusieurs élèves ont travaillé sur un même sujet, regroupez-les et encouragez-les à synthétiser leurs écrits en un seul paragraphe, pour éviter toute confusion ou ennui pour le lecteur.

9 Demandez:

- Quelles sont les caractéristiques d'une bonne rédaction? [Sondez: clarté, éviter les répétitions, bonne organisation, bon vocabulaire, effet affectif approprié, grammaire soignée et bonne orthographe. Écrivez ces qualités au tableau.]

- Comment critiquer de manière constructive le travail d'autrui? [Sondez: mentionner les aspects positifs qu'on apprécie, offrir une critique sensible et respectueuse, faire quelques suggestions mais ne pas récrire le travail de l'autre.]

- Comment accepter positivement la critique? [Sondez: remercier l'autre pour ses suggestions, reconnaître quand on a besoin d'aide, demander une nouvelle critique après un nouvel essai.]
10 Invitez les élèves à critiquer le travail les uns des autres, en consacrant 10 minutes au paragraphe de l'un, 10 minutes à celui de l'autre, puis 10 minutes à l'apport individuel des révisions finales. Annoncez la fin de chaque période de 10 minutes.

11 Ramassez les paragraphes révisés (ainsi que les déclarations personnelles). Affichez tous les flashs d'information sur le mur pour que tout le monde puisse les lire.

S'il vous reste du temps (ou demain) : Invitez quelques étudiants à présenter leur réponse personnelle ou leur flash info final.

Idéalement : Invitez les élèves à compiler leurs flashs d'information, en les agrafant tous ensemble ou en les incluant tous dans un même fichier numérique, puis à les adresser à un journal ou autre débouché d'éducation publique. Encouragezles à présenter le tout sous un titre original et à rédiger une lettre d'accompagnement. 


\section{réflexions sur la grossesse et l'accouchement}

INSTRUCTIONS: Vous allez rédiger deux paragraphes, sur deux feuilles de papier différentes. Le premier doit représenter votre réaction personnelle au sujet choisi : vous n'y exprimerez que vos propres sentiments à l'égard du sujet. Si vous le désirez, parlez-y d'une expérience que vous avez vécue sur la question. Le second paragraphe doit être présenté sous forme de «flash d'information », dans le but d'informer et de sensibiliser d'autres personnes.

\section{POUR PRÉPARER VOTRE FLASH INFO :}

1 Recueillez une information utile sur votre sujet. Pour les sujets A à F, vous trouverez cette information dans le matériel indiqué. Pour les sujets $\mathrm{G}$ à N, interviewez brièvement trois personnes (vos camarades de classe ou d'autres personnes), en veillant autant que possible à obtenir différents points de vue.

2 Expliquez le problème.

3 Décrivez son caractère d'actualité ou la raison pour laquelle il prête à controverse.

4 Rédigez votre conclusion ou terminez par une question qui fasse réfléchir le public. N’oubliez pas que vous écrivez pour une audience publique (les lecteurs d'un journal ou les auditeurs d'une émission de radio).

5 En bas de page, précisez l'origine de votre information : lecture de documentation ou interviews. 


\section{grossesse et accouchement : sujets d'actualité et questions-guides}

\section{LECTURE-RÉFLEXION (A-F)}

A Sélection du sexe dans les endroits où les garçons sont préférés Lire : Fiche d'information sur la sélection du sexe.

Réfléchir : Quelle est la solution à apporter à long terme à ce problème?

B Procréation assistée (technologies d'aide aux couples stériles) Lire : Fiche d'information sur la stérilité et la procréation assistée. Réfléchir : Que pensez-vous de l'idée d'engager et de payer quelqu'un pour porter une grossesse à terme (mère porteuse)?

C Césarienne: Cette intervention chirurgicale n'est pas toujours justifiée, et pas toujours disponible non plus dans les cas où elle serait nécessaire. Où est le juste milieu?

Lire : Fiche d'information sur l'accouchement et l'allaitement maternel (section sur la césarienne).

Réfléchir : Quelle est l'influence des facteurs économiques sur les taux de césarienne?

D Quand l'avortement n'est pas un choix : IVG ou maternité forcées Lire : Fiche d'information sur l'avortement (IVG).

Lire : Section de l'Unité 7 relative à la grossesse non planifiée et à l'avortement (IVG).

Réfléchir : Peut-on forcer qui que ce soit à se faire avorter? Ou à mener à terme une grossesse non désirée?

E Fistule obstétricale

Lire : Fiche d'information sur l'accouchement et l'allaitement maternel (section sur la fistule obstétricale). Réfléchir : Pourquoi ce problème est-il si peu connu ? Comment changer les choses?

F Être enceinte et séropositive à VIH

Lire : Fiche d'information sur l'accouchement et l'allaitement maternel (section sur la grossesse et le VIH).

Lire : Fiche d'information sur la reproduction et la grossesse (section relative à la promotion d'une grossesse saine). Réfléchir : Que pensez-vous de cette question? 


\section{grossesse et accouchement : sujets d'actualité et questions-guides}

\section{INTERVIEW-RÉFLEXION (G-O)}

G Paternité adolescente

Interviewer trois personnes. Demander : Les adolescents sont-ils prêts à être pères ? La plupart restent-ils responsables et actifs dans la vie de leur enfant ? Quelle est la responsabilité des garçons dans la prévention des grossesses non désirées ?

H Faciliter pour les filles la gestion de la menstruation

Interviewer trois filles. Demander : Que font la plupart des filles pour rester propres et absorber leur flux menstruel ? Peut-il y avoir un problème de coût ? Les crampes menstruelles sont-elles problématiques?

| Adoption : Connaître ou non son père, sa mère ou son enfant biologique.

Beaucoup d'enfants sont adoptés par des personnes qui ne connaissent pas leurs père et mère biologiques. L'enfant reçoit parfois une information sur sa mère ou son père biologique ou les deux, et a même parfois des contacts avec eux. Aucune information ou aucun contact n'est parfois disponible ou possible. Interviewer trois personnes. Demander : Vaut-il mieux qu'un enfant soit informé ou non de l'identité de son père ou de sa mère biologique et que l'occasion lui soit donnée ou non de prendre contact avec son père ou sa mère biologique?

J Classes de préparation à la naissance : Préparation des pères et des mères à la naissance et à devenir parents. Dans certains endroits, des classes sont proposées aux couples pour les préparer à l'arrivée d'un enfant. lls y sont informés sur la naissance et sur les soins à apporter au nouveau-né. Interviewer trois personnes. Demander : Ces classes sont-elles nécessaires dans notre communauté ? Quelle différence verrait-on si les pères recevaient cette information?

K Présence des pères à la naissance de leur enfant Interviewer trois personnes. Demander : Est-il utile que les pères assistent à la naissance de leurs enfants ? Cette expérience peut-elle affecter leur lien avec leurs enfants?

L Politiques de congé de maternité et de paternité

Interviewer trois personnes. Demander: Quelle devrait-être la durée du congé de maternité après un accouchement ? Dans certains pays, les pères peuvent aussi bénéficier d'un congé de paternité. Que pensez-vous de cette idée ? Combien de temps les pères devraient-ils pouvoir prendre?

M Qui vous a appris ce que vous savez sur la reproduction et quand? Interviewer trois personnes. Demander : Qui vous a appris d'où viennent les bébés ? Quel âge aviez-vous? Quel est selon vous le meilleur moyen d'apprendre la reproduction humaine?

N Politiques gouvernementales d'incitation sur la taille des familles

Certains États qui désirent voir leur population augmenter offrent des primes aux couples qui ont plus d'enfants. D'autres s'inquiètent de la croissance galopante de leur population et récompensent les individus qui se font stériliser. Interviewer trois personnes. Demander : Êtes-vous d'accord avec ces politiques ? Pourquoi ou pourquoi pas?

\section{Enceinte et à l'école?}

Chaque jour, des adolescentes qui vont encore à l'école se retrouvent enceintes. Dans certains endroits, elles sont autorisées à poursuivre leurs études; dans d'autres, elles doivent quitter l'école. Interviewer trois personnes. Demander: Quelle est la meilleure politique d'appui des adolescentes enceintes et de leur enfant? Quels sont le rôle et la responsabilité du père? 


\section{chaque minute de chaque heure : les histoires de mort maternelle}

APERCU : Les élèves lisent les récits de décès maternels. Par mise en scène et discussion, ils explorent la manière dont ces décès auraient pu être évités.

OBJECTIFS: Aider les élèves à décrire comment la pauvreté et l'inégalité de genre mènent à la mort (ou à de graves problèmes de santé) chez les femmes enceintes; identifier les moyens de prévention possibles ; les sensibiliser au problème de la mortalité maternelle ; renforcer la réflexion analytique.

\section{DURÉE :}

60-85 minutes, suivant le nombre d'études de cas retenu

\section{MATÉRIEL :}

Tableau noir + craie ; une copie de chaque « étude de cas de mortalité maternelle ».

\section{PRÉPARATION :}

\section{Lisez les huit études de cas} proposées et modifiez ou remplacez-les, si nécessaire, pour les adapter ou les valider dans votre contexte. Consultez les sections pertinentes (unité 6, «Quand la grossesse se poursuit » et les fiches sur la reproduction et la grossesse et l'accouchement et l'allaitement maternel) du GUIDE compagnon sur $<$ www.unseulprogramme. org> ou référez-vous à une autre source d'information fiable.

\section{INSTRUCTIONS}

1 Écrivez les mots « mortalité maternelle » au tableau. Posez les questions suivantes et écrivez les réponses au tableau :

- Comment définiriez-vous la «mortalité maternelle »? [Sondez pour une définition du type: " la mort d'une femme due à une cause liée à une grossesse ou un accouchement $»$.]

- Essayez maintenant de répondre à ces questions : Arrive-t-il souvent qu'une femme ou une fille meure, quelque part dans le monde, de causes liées à la grossesse? Une fois par semaine ? Tous les jours? Toutes les 10 minutes? [Invitez quelques essais.] La réponse correcte est chaque minute de chaque jour, chaque jour de l'année. Pendant l'heure que nous allons passer à en parler, 60 femmes et filles seront mortes. Cela fait un demimillion par an. Bien plus encore survivent mais souffrent de séquelles liées à leur grossesse.

- Pensez-vous que la mortalité maternelle est égale partout dans le monde ? Quelle proportion placeriez-vous dans les pays «en développement » (les pays non encore industrialisés ou riches) ? Un dixième? La moitié ? Deux tiers? [Invitez quelques essais.] La réponse est 99 pour cent. La mortalité maternelle est extrêmement rare dans les pays plus « développés ».

- Le risque est plus grand aussi pour les jeunes adolescentes. Par rapport aux femmes adultes, celles de moins de 15 ans meurent beaucoup plus souvent en couches.

- Combien de décès maternels pourraient-être évités ? Très peu ? Plus de la moitié ? Un tiers? [Invitez quelques essais.] D’après les experts, " presque tous » ces décès sont évitables.

- Le risque de mortalité maternelle est-il plus ou moins grand aujourd'hui qu'il y a quelques décennies? [Invitez quelques essais.] Le fait est que les risques sont à peu près égaux.

- Nous allons parler aujourd'hui de ces questions, pour vous aider à déterminer comment changer les choses, pour vous-mêmes et pour d'autres. 
2 Formez autant de groupes que vous avez d'études de cas. Distribuez une étude à chaque groupe (pliez la feuille pour qu'ils ne commencent pas encore à lire.) Expliquez :

- Chaque groupe a reçu une étude de cas de mortalité maternelle différente. Lisez la vôtre et discutez-la avec votre groupe, en vous demandant: Que s'est-il passé? (Quels sont les principaux facteurs qui ont contribué ou mené à la mort de la femme ou de la fille ?) Qu'aurait-il dû se passer? (Comment les choses auraientelles pu tourner autrement et lui sauver la vie ?) [Écrivez ces deux questions au tableau.]

- Vous allez interpréter l'histoire de ces filles et femmes sous forme de petites scènes (2 à 3 minutes), d'abord selon le texte écrit, puis en y incorporant les démarches qui auraient permis de leur sauver la vie. Les mêmes acteurs peuvent jouer les deux scènes, ou d'autres membres du groupe peuvent interpréter la seconde.
3 Invitez tous les groupes à interpréter leurs deux scènes.

4 Réservez 15 minutes, à la fin, pour discuter :

- Quels sont vos sentiments face à ces récits ? Pourquoi?

- Chaque personne, les membres de la famille, les communautés, les prestataires de soins et les gouvernements peuvent tous agir pour prévenir ces décès. Quelles sont les principales mesures, avant qu'une femme ne soit enceinte, qui protégeraient sa santé (et celle de son enfant)? [Écrivez, et complétez, les réponses au tableau.]

- Que faire pendant la grossesse? [Écrivez, et complétez, les réponses au tableau.]

- Que faire pendant l'accouchement? [Écrivez, et complétez, les réponses au tableau.]

- Comment prévenir la mortalité maternelle parmi les filles de moins de 15 ans?

- Comment allez-vous utiliser ce que vous avez appris aujourd'hui ? 


\section{études de cas de mortalité maternelle}

L'HISTOIRE DE RANI : Quand Rani avait 13 ans, sa mère est morte en couches. Son père lui a expliqué qu'il fallait qu'elle quitte l'école pour faire la cuisine et s'occuper de ses jeunes frères et sœurs. Resté à l'école, son frère aîné a essayé d'aider Rani à poursuivre ses études, mais elle a bientôt dû renoncer, car ses tâches ménagères ne lui laissaient guère de temps. À 19 ans, Rani a épousé Ramesh et est allée vivre avec lui et ses parents. La famille de Ramesh appréciait l'expérience de Rani à s'occuper d'enfants et voulait qu'elle en ait sans tarder.

Quand Rani s'est retrouvée enceinte, son mari ne lui parlait que d'un fils. En secret, elle imaginait pourtant avoir une fille, et elle se promettait que sa fille serait autorisée à achever ses études, comme elle ne l'avait jamais été. Au bout de huit mois, selon la coutume locale, Rani est revenue au village de ses parents pour accoucher. Quand les douleurs du travail ont commencé, un médecin local est venu la voir. Après toute une journée et une nuit de travail, il l'a envoyée à l'hôpital le plus proche, à 100 kilomètres. Personne ne pouvait cependant l'y conduire avant le matin. Enfin arrivée à l'hôpital, Rani, épuisée, a accouché. Face au regard déçu de son mari, Rani a murmuré sa promesse à sa fille. Mais elle s'est mise à saigner abondamment et, tandis que le personnel hospitalier cherchait à lui faire une transfusion d'urgence, Rani est tombée dans le coma. Malgré les efforts de l'hôpital, Rani avait perdu beaucoup de sang. Elle est morte comme sa mère avant elle.

L'HISTOIRE DE CHARITY : Charity avait 15 ans. Elle allait à l'école ; elle étudiait et elle travaillait aux tâches du ménage. Elle était sortie quelques fois avec un homme qu'elle avait rencontré près de l'école. Il lui avait donné des cadeaux et un peu d'argent, avec lequel elle avait acheté ses livres scolaires, et elle avait eu des rapports sexuels avec lui. Elle lui avait parlé du préservatif, comme elle l'avait appris à l'école, mais il lui avait dit de ne pas s'inquiéter. Elle ne l'aimait pas trop et a donc décidé de ne plus le revoir. Charity a cependant bientôt découvert qu'elle était enceinte. Quand elle l'a dit à ses parents, son père l'a battue mais ne l'a pas mise à la porte. Au bout de quelques mois, elle a abandonné l'école pour aller vivre avec une tante, dans une autre ville. Sa famille avait décidé que la tante élèverait l'enfant et que personne ne saurait rien de la grossesse de Charity. Quand son travail a commencé, sa tante lui a dit de rester forte, que l'enfant viendrait de lui-même. Mais il est resté bloqué et la tante a dû appeler une accoucheuse, qui a élargi l'orifice du vagin à l'aide d'une lame de rasoir. L'enfant est alors né rapidement et Charity a pensé qu'elle reprendrait bientôt sa vie d'avant, plus triste mais aussi plus sage. En l'espace de quelques jours, l'endroit de la coupure s'est cependant infecté et, après quelques jours encore, Charity a été prise d'une forte fièvre. Sa tante a voulu l'emmener à l'hôpital mais elle a refusé, de peur que quelqu'un se rende compte qu'elle avait été enceinte. Le lendemain, comme la fièvre avait encore monté, la tante a quand même appelé l'ambulance mais il était trop tard. Charity est morte quelques heures après son arrivée à l'hôpital. 


\section{études de cas de mortalité maternelle}

L'HISTOIRE DE ANA : Ana vivait avec son mari dans un village isolé des Andes, en Bolivie. Elle n'avait jamais appris à lire ni à écrire.Elle avait pris la pilule contraceptive pendant quelque temps mais avait arrêté car son mari y était opposé. Quand elle est tombée enceinte, Ana est allée une fois au centre de santé local pour une visite de soins prénatals. Gênée de ne pas parler espagnol face à un personnel qui ne parlait pas le quechua, elle ne s’y est pas sentie bienvenue. Durant le neuvième mois de sa grossesse, Ana a commencé à sentir des douleurs dans le bas du ventre. Une voisine accoucheuse a dit à sa famille que le bébé se présentait mal et qu'il fallait qu'ils l'emmènent au centre de santé. Ana craignait d'y être traitée comme elle l'avait été lors de sa première visite. Son mari avait de plus entendu dire que les services du centre étaient payants et sa famille n'en avait pas les moyens. Ana est donc restée chez elle. Au bout de 10 jours, la douleur a empiré et l'accoucheuse a persuadé la famille d'Ana de l'emmener au centre médical. N'ayant pas accès à une voiture, ils l'ont transportée en chariot tiré par un cheval. Le centre se trouvait à 15 kilomètres du village, une longue route en chariot. À mi- chemin, Ana s'est mise à saigner abondamment et est morte.

L'HISTOIRE DE FATIMA : Fatima rêvait de devenir institutrice. Mais à l'âge de 15 ans, ses parents l'ont mariée et elle est allée vivre avec son mari, Ali, qui était routier. Les parents d'Ali ont fait pression pour qu'ils aient des enfants. Fatima n'a donc pas utilisé la contraception. Elle a accouché de trois enfants en cinq ans, avec l'aide d'une sage-femme locale. Au huitième mois de sa quatrième grossesse, tandis qu'Ali était au travail, Fatima a commencé à se sentir très mal. Comme Ali gérait l'argent de la famille, elle n'avait pas de quoi payer son transport au dispensaire. Elle a donc attendu qu'il rentre. Quand il est arrivé, Fatima tremblait de fièvre et était extrêmement faible. Ali a pris les dispositions pour l'emmener à l'hôpital, mais elle est morte avant d'y arriver.

L'HISTOIRE DE MARIA CLARA : Maria Clara vivait avec son mari, Pedro, et son neveu (le fils de son frère décédé lors d'un accident). Ils vivaient dans un village de montagne, bien plus haut que la ville, à sept heures de route à dos d'âne de l'hôpital le plus proche. Quand Maria Clara est tombée enceinte, elle est allée faire une visite médicale. Le médecin lui a dit de venir à l'hôpital deux semaines avant la date prévue de l'accouchement pour éviter toutes complications graves de dernière minute. Vers la fin de sa grossesse, Maria Clara est devenue anxieuse à l'idée de quitter sa maison. Pedro redoutait une si longue absence, se plaignant de ne pas savoir cuisiner. Elle savait aussi que Pedro buvait presque tous les soirs et qu'il était parfois violent, et elle craignait qu'il ne s'en prenne à son neveu. Tout cela sans compter que Pedro risquait de passer du temps avec une femme qui travaillait au bar où il se rendait après le travail : une voisine lui avait dit que la serveuse avait été vue avec Pedro. Maria Clara a donc décidé de ne descendre à l'hôpital qu'une semaine avant la date d'accouchement prévue par le médecin. L’enfant est cependant né la veille de son départ et Maria Clara s'est mise à saigner. Pedro n’a pas pu arriver au centre de santé à temps pour la sauver. 


\section{études de cas de mortalité maternelle}

L'HISTOIRE DE YERUKNESH : Yeruknesh était née dans une communauté isolée d'Éthiopie. À l'âge de 13 ans, alors qu'elle ramassait du bois à brûler, elle a été enlevée puis mariée à $\mathrm{M}$. Zena, un veuf de 32 ans père de deux enfants. Peu après, elle s'est retrouvée enceinte. Yeruknesh n'avait personne à qui demander conseil ou à qui parler de ses problèmes. Quand son travail a commencé, terrifiée, elle s'est accrochée à son oreiller, appelant sa mère, le visage couvert de larmes. Elle souffrait l'agonie, avait de la fièvre et suait abondamment. Quelques voisines sont venues, ont allumé une bougie et prié pour elle. M. Zena a fait appel à l'accoucheuse traditionnelle du village, incapable de faire face à la situation. M. Zena a alors demandé aux hommes du village de transporter Yeruknesh à l'hôpital. Son enfant mort-né, Yeruknesh est morte aussi, à l'âge de 15 ans.

L'HISTOIRE DE HUSAN : Saeeda Bibi, qui vit à Mardan dans la province du Nord-Ouest du Pakistan, raconte l'histoire de sa fille unique, Husan Pari, morte en couches à l'âge de 13 ans. "Quand Husan avait huit ans, son père est mort d'un accident de la route. J'avais peur : si je mourais aussi, qui s'occuperait de ma fille ? Je voulais la marier, qu'elle soit heureuse et en sécurité. Je lui ai trouvé un mari, agriculteur, dans un village proche. Husan Pari était si excitée à l'idée de se marier. Après trois mois, elle était enceinte et je l'ai emmenée chez l'accoucheuse traditionnelle, qui lui a donné des remèdes pour l'empêcher de vomir. Nous pouvions payer la Dai en plusieurs fois et nous aimions ses prières et ses remèdes. Husan travaillait très dur mais elle mangeait mal (elle ne voulait plus d'œufs ni de poisson, par superstition) et elle était terriblement maigre. Je m'inquiétais pour l'accouchement. Nous sommes allées l'aider quand elle s'est mise à crier, mais le bébé ne venait pas. L'accoucheuse est devenue nerveuse, n’a plus voulu aider et a dit qu'il fallait l'emmener à l'hôpital. En chemin, le corps d'Husan Pari est devenu bleu et froid et elle est morte dans mes bras. Je n'ai pas pu la sauver ni son enfant. Je ne sais plus comment nous sommes rentrés, ou comment les funérailles ont eu lieu. La douleur était insupportable. »

Les histoires de Yeruknesh et d'Husan sont adaptées de White Ribbon Alliance for Safe Motherhood and Stories of Mothers Lost, avec la permission de l'organisation. Voir $<$ www.whiteribbonalliance.org/ exhibit/default.cfm>. L'histoire de Yeruknesh vient de Medico Socio Development Assistance of Ethiopia, et celle d'Husan, de Blue Veins - Women Welfare and Relief Service of Pakistan. Plusieurs des autres récits sont inspirés d'histoires vraies, sous des noms de femmes parfois différents.
L'HISTOIRE DE FLORENCE : Florence était une jeune femme de 24 ans qui cultivait et vendait des légumes. Un soir, un homme l'a violée sur le chemin du retour du marché. Effrayée, elle n’a rien dit à personne mais quand ses règles ne sont pas venues ce mois-là, elle a compris qu'elle était enceinte. L'avortement n'était pas libre dans son pays et il se pratiquait généralement dans des conditions peu sanitaires, mais Florence était résolue à interrompre sa grossesse. Elle est allée voir quelqu'un qui lui a introduit quelque chose dans le vagin et lui a dit que le saignement s'arrêterait en l'espace d'une semaine. Au bout de la semaine, Florence saignait encore et elle avait beaucoup de fièvre. Elle est allée à l'hôpital, où un médecin lui a donné des antibiotiques. Ne voulant pas révéler qu'elle s'était fait avorter, de peur d'être punie ou même arrêtée, Florence a quand même fini par le dire au médecin. Celui-ci était en fait d'avis que l'avortement devrait être sûr et accessible. Il était profondément désolé pour Florence et a fait de son mieux pour la sauver. Il n'a pas pu retenir ses larmes quand elle est morte 10 jours plus tard. 


\section{les faits sur les infections sexuellement transmissibles (IST)}

APERCQU : Les élèves créent des affiches présentant l'information importante à savoir sur les IST, y compris le VIH et le sida.

OBJECTIFS : Aider les élèves à décrire comment les IST se transmettent, comment prévenir leur transmission et où se faire tester et traiter ; les sensibiliser à l'importance du dépistage et du traitement.

\section{INSTRUCTIONS}

1 Expliquez que cette leçon concerne les infections sexuellement transmissibles — les IST - y compris le VIH et le sida.

2 Divisez la classe en quatre groupes mixtes. Donnez à chacun un sujet de la liste (ou laissez-les choisir ; essayez de couvrir tous les sujets.) Expliquez:

- Chaque groupe va créer une affiche informative sur son sujet. Faites preuve d'imagination!

- Présentez une information complète : couvrez le dépistage, le traitement et la prévention. Servezvous de la documentation. Pour les affiches sur les « mythes ", notamment, il vous sera aussi utile d'interviewer vos camarades de classe.

3 Distribuez la documentation factuelle et le matériel. Dites aux élèves qu'ils ont 10 minutes pour recueillir leur information, 10 minutes pour discuter et planifier leur affiche et 15 minutes pour la réaliser. Circulez parmi les groupes pour les aider pendant toutes ces phases. S'ils n'ont pas fini, donnez-leur plus de temps ou dites-leur quand ils pourront achever leur travail.

Lors d'une session ultérieure

4 Au besoin, donnez aux groupes le temps d'achever leur affiche.

5 Invitez chaque groupe à présenter son affiche et à répondre aux questions de la classe.

6 Gardez les affiches au mur de la classe aussi longtemps que possible.

\section{THËMES}

Les mythes sur les IST

Les mythes sur le VIH et le sida

La double protection

Cinq faits importants sur le VIH et le sida

Qu'est-ce que la chlamydia?

Les IST et le genre

Comment la chlamydia et la blennorragie affectent la fécondité

HPV et herpès : deux IST répandues

Qu'est-ce qu'une infection de l'appareil génital ?

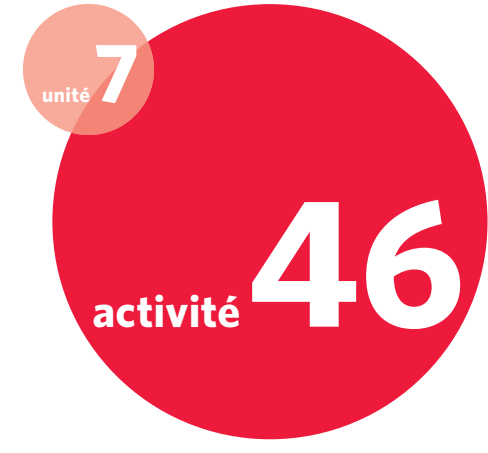

\section{DURÉE :}

Étapes 1-3: 40 minutes

Étapes 4-6: 40 minutes

\section{MATÉRIEL :}

Papier affiche + marqueurs ; volume GUIDE (compagnon de de ce volume) ou copies des pages suivantes du guide : fiches d'information sur les infections sexuellement transmissibles, le VIH et le sida et les infections de l'appareil génital, et sections de l'unité 7 sur les IST et le VIH. Le volume GUIDE est accessible sur <www. unseulprogramme.org>. Ou bien autre source d'information fiable sur les IST et le VIH.

\section{PRÉPARATION :}

Si possible, faites plusieurs copies du matériel listé cidessus. Informez-vous sur les endroits où les jeunes peuvent se faire tester et traiter dans votre communauté. Écrivez les thèmes d'affiche au tableau. 


\section{le genre et le préservatif}

\section{DURÉE :}

Étapes 1-4 (Pourquoi certains refusent le préservatif) : 40 minutes

Étapes 5-7 (Usage correct du préservatif) : 15 minutes

\section{MATÉRIEL :}

Tableau noir + craie ; « Clés pour l'enseignant »; pour les étapes 1-4: copies des feuilles de travaille à distribuer. Pour les étapes 5-7, cartes-repères. Si approprié, préservatif masculin et féminin pour démonstration.

\section{PRÉPARATION :}

Renseignez-vous sur la

disponibilité locale du préservatif féminin. Lisez l'information sur le préservatif masculin et féminin sur la fiche d'information relative à la contraception à la fin du GUIDE compagnon de ce volume ou référez-vous à un autre texte fiable. Pour les étapes 1-4 : Lisez la feuille de travaille et les deux clés pour l'enseignant. Pour les étapes 5-7 : Préparez les cartes (voir

« Usage correct du préservatif »)
APERCUU : Les élèves analysent les obstacles (normes de genre comprises) à l'usage du préservatif et les moyens de les surmonter. Ils apprennent aussi comment utiliser le préservatif. Cette activité s'adresse aux élèves déjà informés sur le préservatif en tant que méthode de protection contre les IST, le VIH et les grossesses non désirées.

OBJECTIFS: Apprendre aux élèves à décrire les obstacles à l'usage du préservatif et comment les résoudre ; décrire l'usage correct du préservatif ; renforcer la réflexion critique.

\section{INSTRUCTIONS}

1 Expliquez (et écrivez les réponses clés) :

- Nous allons parler aujourd'hui du préservatif. Que savons-nous du préservatif? [Sondez:Il protège contre les IST, le VIH et les grossesses non désirées; méthode masculine.]

- Qui peut utiliser le préservatif ? Existe-t-il pour les femmes, ou juste pour les hommes? [Sondez : Il existe aussi un préservatif féminin. Présentez une information générale sur cette méthode.]

- Pourquoi est-il important d'être informé sur le préservatif? [Sondez: Chaque jour des gens meurent du sida et de complications de l'avortement non médicalisé; ces décès auraient pu être évités si le préservatif avait été utilisé. Le préservatif protège contre la grossesse, le VIH et d'autres IST.]

- Quand on sait que le préservatif existe, cela veutil dire qu'on l'utilise?
2 Organisez les élèves deux par deux et distribuez la feuille d'exercice. Expliquez :

- Il y a beaucoup de raisons pour lesquelles le préservatif n'est pas utilisé ou ne l'est pas correctement. Cette feuille décrit l'expérience de neuf couples réels et suggère les solutions qui auraient pu leur être utiles.

- Avec votre partenaire, lisez la situation de chaque couple et identifiez la ou les solutions qui leur conviendraient : que pourraient-ils faire pour utiliser le préservatif correctement la prochaine fois?

3 Rassemblez la classe et discutez la situation de chaque couple décrit. Pour les couples C, F et I (où il y a inégalité de genre), approfondissez la discussion si vous en avez le temps.

4 Après avoir considéré la situation des neuf couples, posez ces questions pour animer la discussion : 
- À qui revient la responsabilité du préservatif ?

- Pourquoi les filles ou les femmes doivent-elles avoir accès au préservatif féminin? [Sondez: pour assumer elles-mêmes leur protection si leur partenaire refuse le préservatif masculin.]

- Comment s'assurer qu'on est prêt à utiliser le préservatif quand on en a besoin?

5 Divisez la classe en deux ou trois équipes (environ 12 à 16 élèves par équipe) et présentez le sujet :

- Une des raisons pour lesquelles on n'utilise pas le préservatif est qu'on ne sait pas comment l'utiliser. C'est là une bien mauvaise raison d'en arriver à un grave problème de santé. Nous allons donc apprendre comment utiliser adéquatement le préservatif masculin. Être suffisamment grand pour savoir comment utiliser le préservatif ne veut PAS dire qu'on est prêt à avoir des rapports sexuels, mais il vaut mieux le savoir AVANT d'en avoir besoin plutôt qu'après, quand il est trop tard. Certaines démarches sont utiles bien avant de se trouver dans une situation intime. D'autres, quand on se trouve déjà dans cette situation, juste avant les rapports sexuels en soi. Il faut savoir comment utiliser le préservatif pendant les rapports, et savoir aussi qu'en faire après usage. [Au tableau, écrivez "bien à l'avance ", "juste avant les rapports sexuels", "pendant » et « après ».]
6 Expliquez l'activité :

- J'ai identifié toutes les étapes nécessaires à l’usage correct du préservatif masculin et je les ai écrites individuellement sur différentes cartes, dont je vais distribuer une série à chaque groupe. [Veillez à mélanger les cartes.] Chaque équipe a pour tâche de remettre les cartes dans le bon ordre.

- Si possible, faites en sorte que chaque membre de votre groupe ait une carte, puis organisezvous en quatre sous-groupes. [Montrez les quatre options au tableau.] Dans le premier, placez tous les membres du groupe dont la carte concerne les premières étapes (celles à effectuer bien avant l'acte sexuel) ; dans le deuxième, ceux dont les cartes concernent les étapes suivantes, juste avant les rapports ; dans le troisième, rassemblez les étapes à accomplir pendant les rapports sexuels et dans le quatrième, celles devant intervenir après l'acte. Dans chaque sous-groupe, déterminez l'ordre des cartes et formez un rang le représentant. [Remarque: L'ordre est difficile à établir dans le premier groupe.]

- Allez-y! Vous avez 5 minutes.

7 Arrêtez l'activité au bout de 5 minutes et rassemblez le groupe au complet. Invitez les élèves à expliquer l'ordre des étapes. Interrompez et interrogez-les si l'ordre est incorrect (voir les clés). Répondez aux questions posées. 


\section{solutions pour un usage gagnant du préservatif}

Les paragraphes ci-dessous décrivent la situation de neuf couples qui n'ont pas bien utilisé le préservatif. Pour chacun, réfléchissez à ce qui aurait aidé la personne ou le couple. Choisissez une ou plusieurs « solutions » dans la liste proposée et entourez le ou les chiffres correspondants. Par exemple, entourez « 2 » pour indiquer « Plus grande égalité et pouvoir partagé entre la fille et le garçon ». (Entourez autant de chiffres qu'il y a de solutions possibles pour chaque cas.)

$\begin{array}{ll}\text { Solution } 1 & \text { Information sur l'usage correct du préservatif } \\ \text { Solution } 2 & \text { Plus grande égalité et pouvoir partagé entre la fille et le garçon } \\ \text { Solution } 3 & \text { Meilleures aptitudes à communiquer } \\ \text { Solution } 4 & \text { Information sur l'endroit où se procurer le préservatif } \\ \text { Solution } 5 & \text { Idée plus réaliste du risque que représentent le VIH et la grossesse } \\ \text { Solution } 6 & \text { Lieu sûr où parler de ses inquiétudes de performance sexuelle }\end{array}$

Couple A : Deux jeunes ont des rapports sexuels, avec préservatif. Après avoir éjaculé, le garçon reste là, sans bouger, pendant cinq minutes. Son pénis se ramollit et se rapetisse et, quand il bouge enfin, il s'étonne de voir qu'un peu de son sperme s'écoule hors du préservatif à l'entrée du vagin de son amie.
Solution(s) pour le couple A :
1
2
3
4
5
6

Couple B : Deux jeunes ont décidé d'avoir des rapports sexuels et d'utiliser le préservatif. L’un des partenaires va au magasin mais ne trouve ni le préservatif masculin, ni le féminin. L'autre pense pouvoir s'en procurer à l'école, mais l'infirmière lui dit que non. Chacun espère que l'autre en a trouvés. Le week-end suivant, ils passent à l'acte. Ils sont tous deux gênés de ce qui leur est arrivé, ignorent donc la question et ont des rapports sans préservatif.
Solution(s) pour le couple B :
1
2
3
4
5
6

Couple C : Deux jeunes veulent avoir des rapports sexuels. La fille dit au garçon qu'elle a apporté des préservatifs mais il dit : «Pas question! Ces machins sont horribles! » Elle essaie d'insister mais il se fâche, lui dit qu'elle se comporte comme une gamine et lui demande si elle l'aime vraiment. Elle finit par céder.

Solution(s) pour le couple $\mathrm{C}$ : 


\section{solutions pour un usage gagnant du préservatif}

Couple D : Un garçon et une fille veulent avoir des rapports sexuels et ont décidé d'utiliser le préservatif. Le moment venu, le garçon cafouille avec l'emballage et perd son érection. Le couple renonce cette fois aux rapports. La fois suivante, nerveux à l'idée de perdre de nouveau son érection, il dit à sa petite amie que le préservatif détruit l'atmosphère et ils décident donc d’avoir des rapports non protégés.
Solution(s) pour le couple D :
2
4
5
6

Couple E : Un couple décide d'avoir des rapports sexuels. Les deux partenaires pensent qu'il serait bon d'utiliser le préservatif mais chacun a peur que l'autre pense qu'il ou elle a «l'esprit mal tourné » s'il ou elle le propose. En fin de compte, ni l'un ni l'autre n'aborde la question et ils ont des rapports sans préservatif.
Solution(s) pour le couple E :
1
2
3
4
5
6

Couple F : Un garçon de 16 ans veut quitter le gang dont il fait partie mais cela peut être difficile et dangereux, à moins cependant qu'il ne devienne père. Il explique à sa petite amie de 15 ans que s'ils font un enfant, cela pourrait lui sauver la vie et qu'il sera un bon père. Elle est déconcertée. Elle voudrait achever ses études et ne se sent pas prête à être mère. Mais elle pense aussi qu'elle doit aider son petit ami. Ils ont des rapports sexuels sans préservatif
Solution(s) pour le couple F :
1
2
3
4
5
6

Couple G : Un garçon et une fille décident d'avoir des rapports sexuels. Le garçon demande s'ils devraient utiliser une protection mais elle dit qu'elle vient d'avoir ses règles et ne risque donc pas de tomber enceinte.
Solution(s) pour le couple $\mathbf{G}$ :
1
2
3
4
5
6

Couple H : Un couple décide d'avoir des rapports sexuels. Il parle du préservatif pour se protéger du VIH mais détermine que chacun saurait s'il était malade. Le couple a donc des rapports non protégés.
Solution(s) pour le couple $\mathrm{H}$ :
1
2
3
4
5
6

Couple I : Une fille de 17 ans a des rapports sexuels avec un homme de 25 ans qui lui fait des cadeaux et lui donne parfois de l'argent pour couvrir ses dépenses. Il utilise parfois le préservatif, mais il n'en a pas aujourd'hui. Elle pense qu'ils devraient attendre et avoir des rapports une autre fois, mais il lui promet qu'il n’y a pas de risque. Elle a déjà accepté son argent cette semaine et pense donc ne pas pouvoir refuser. Ils ont des rapports sans préservatif.
Solution(s) pour le couple I :
1
2
3
4
5
6 


\title{
« solutions pour un usage gagnant du préservatif » - clé
}

REMARQUE AUX ENSEIGNANTS : Les « solutions » principales aux problèmes des couples de chaque situation sont listées ici. Vos élèves en suggéreront peut-être d'autres. Soyez ouvert à leurs réponses mais veillez, au minimum, à ce que les solutions ci-dessous soient mentionnées.

\author{
Couple A Solution 1 (information sur l'usage correct du préservatif) \\ Couple B Solution 4 (information sur l'endroit où se procurer le préservatif) et \\ Solution 3 (meilleures aptitudes à communiquer) \\ Couple C Solution $\mathbf{2}$ (plus grande égalité et pouvoir partagé entre la fille et le garçon) et \\ Solution 3 (meilleures aptitudes à communiquer) \\ Couple D Solution 6 (lieu sûr où le garçon puisse parler de son anxiété de performance) \\ Couple E Solution 3 (meilleures aptitudes à communiquer) et \\ Solution 5 (idée plus réaliste du risque que représentent le VIH et la grossesse) \\ Couple F Solution 2 (plus grande égalité et pouvoir partagé entre la fille et le garçon) \\ Couple G Solution 5 (idée plus réaliste du risque que représente la grossesse) \\ Couple $\mathbf{H} \quad$ Solution $\mathbf{5}$ (idée plus réaliste du risque que représente le VIH) \\ Couple I Solution 2 (plus grande égalité et pouvoir partagé entre la fille et le garçon)
}




\section{usage correct du préservatif}

REMARQUE AUX ENSEIGNANTS : Préparez 16 cartes-repères. Sur chacune, écrivez une étape de la liste ci-dessous, sans indiquer le «numéro » de l'étape. (Les numéros indiqués dans la liste sont votre clé.) Pour rappel, l'ordre des étapes 1 à 5 n'est pas nécessairement fixe.

\section{(Bien à l'avance)}

1 Parler des pratiques sexuelles sans risques avec son/sa partenaire.

2 Acheter des préservatifs (et peut-être aussi un lubrifiant) ou trouver une clinique ou un centre communautaire qui les distribue gratuitement.

3 Garder ses préservatifs en lieu sec et frais (pas dans un portefeuille).

4 Vérifier la date d'expiration du préservatif et s'assurer qu'elle n'est pas dépassée.

5 S'exercer à mettre le préservatif pour se sentir à l'aise le moment venu.

(Juste avant les rapports sexuels)

6 Engager les préliminaires qui, avec la stimulation du clitoris, peuvent aider à lubrifier le vagin.

7 Ouvrir délicatement l'emballage, en veillant à ne pas déchirer le préservatif (ne pas l'ouvrir avec les dents !)

8 Quand le pénis est en érection ... serrer le bout du préservatif entre les doigts et poser le préservatif enroulé au bout du pénis.

9 Tenir le bout et dérouler le préservatif jusqu'à couvrir complètement le pénis.

10 Si le vagin semble sec, continuer les préliminaires ou mouiller l'extérieur du préservatif à l'aide d'un lubrifiant aqueux ou d'un peu de salive. Ne jamais utiliser de Vaseline ${ }^{\circledR}$ ou d'autres produits à base d'huile, car ils affaiblissent la résistance du préservatif et peuvent causer sa déchirure.

(Pendant les rapports sexuels)

11 Si le préservatif se déchire, l'homme doit se retirer immédiatement. [Peut-être voudrez-vous rappeler aux élèves de considérer la contraception d'urgence si l'éjaculation a déjà eu lieu.]

12 Après l'éjaculation, tant que le pénis est toujours en érection.

13 Tenir l'extrémité ouverte du préservatif à la base du pénis.

(Immédiatement après les rapports sexuels)

14 En tenant le préservatif à la base du pénis, retirer doucement le pénis du vagin.

15 Retirer soigneusement le préservatif sans renverser de sperme, en tenant l'anneau du préservatif.

16 Nouer le préservatif ou l'enrouler dans du papier hygiénique et s'en débarrasser de manière appropriée. 


\section{entamer la conversation sur le sexe et la sécurité}

APERC̣U : Les élèves s'exercent à entamer la conversation sur d'importantes questions de sécurité et de santé sexuelles souvent omises. (Remarque : Cette activité et l'activité 38 se recoupent ; il peut être utile d'en choisir une ou les deux.)

OBJECTIFS : Aider les élèves à réfléchir et à s'exercer à entamer et tenir une conversation sur les sujets du sexe et de la sécurité ; renforcer la réflexion critique.

\section{DURÉE :}

Étapes 1-3: 40 minutes

Étapes $4-10: 40$ minutes

\section{MATÉRIEL :}

Tableau noir + craie

\section{PRÉPARATION :}

Lisez et, au besoin, modifiez les scénarios (le couple pourrait par exemple être fiancé). Minutez

soigneusement l'activité et gardez le jeu de rôles bref.

\section{INSTRUCTIONS}

1 Présentez l'activité en posant les questions-guides suivantes :

- Aujourd'hui, nous allons imaginer et pratiquer la conversation sur la sécurité sexuelle. Pour cet exercise, nous allons nous concentrer sur les conversations relatives aux échanges hétérosexuels. Est-il facile de parler de questions de nature sexuelle? Pourquoi ?

- Comment faciliter les choses?

2 Invitez les élèves à s'organiser deux par deux (garçonfille, de préférence). Écrivez au tableau :

Avoir des rapports sexuels ou non

Expérience sexuelle antérieure

Infections sexuellement transmissibles, VIH et sida

Usage de drogues antérieur

Utilisation du préservatif

3 Expliquez:

- Exercez-vous avec votre partenaire à entamer la conversation sur des questions difficiles mais importantes. Pour chaque sujet, discutez comment entamer la conversation avec un(e) partenaire sexuel(le) potentiel(le). Écrivez au moins une approche spécifique.

- Décidez aussi du moment où la première conversation doit avoir lieu : lors de la première rencontre? Après le premier baiser? En pleine situation sexuelle? N'oubliez pas qu'il n'est pas nécessaire d'aborder tous les sujets à la fois.

4 Pour chaque sujet, invitez un couple à partager ses idées. Écrivez ses réponses au tableau. Demandez:

- Quelqu'un a-t-il d'autres suggestions? (Ajoutez-les à la liste.)

- Quelles idées vous semblent utiles et pourquoi ? Certaines suggestions vous paraissent-elles moins bonnes que d'autres? Pourquoi ?

- Quand, dans une relation, cette première conversation doit-elle avoir lieu ? Pourquoi?

5 Après avoir passé en revue les cinq sujets, demandez :

- Que faire pour faciliter ce genre de conversation?

- Voyons maintenant comment ces conversations pourraient se dérouler dans la réalité. 
6 Expliquez:

- La première personne entame la conversation. Mais la seconde rend le dialogue difficile : elle se montre mal à l'aise, n'est pas d'accord, ou essaie d'éviter la conversation.

- La tâche de la première personne est de faire progresser, tant soit peu, la conversation.

7 Invitez deux volontaires à interpréter une conversation sur le premier sujet (rapports sexuels ou non). Expliquez :

- Donnez un nom à vos personnages et essayez d'être réalistes.

- Le scénario est le suivant : [Insérez les noms choisis.]

- «Ali et Lia sortent ensemble depuis un bout de temps et commencent à se sentir physiquement intimes. Ils n'ont pas encore eu de rapports sexuels. Ils ne sont pas sûrs des antécédents sexuels ou de drogue l'un de l'autre. Ali croit qu'ils pourraient s'engager davantage sexuellement mais il a vraiment peur du VIH. Le couple se promène. »

- Invitez les spectateurs à prendre note de la manière dont les deux volontaires communiquent.

8 Animez une brève discussion, en vous aidant des questions suivantes :

- Quels sont les points positifs ? Qu'auraient-ils pu aborder différemment?
- Cette conversation était-elle réaliste ?

- Avez-vous des conseils à donner à Ali ou à Lia ?

9 Répétez les étapes 6 à 8 pour autant des scénarios suivants que possible :

- Anjali entame la conversation avec Mo sur la question d'avoir ou non des rapports sexuels. Ils peuvent ou non être du même avis.

- Carlo entame la conversation avec Mar sur leur expérience sexuelle et usage de drogue passés.

- Henry et Mia en ont parlé et pensent vouloir avoir des rapports sexuels. Henry entame la conversation sur le préservatif. [En privé, dites à Henry qu'il ne veut pas avoir de rapports sans préservatif, et à Mia qu'elle ne pense pas que le préservatif soit nécessaire.]

10 Pour conclure, posez les questions suivantes et écrivez les réponses clés au tableau :

- Avant d'avoir une conversation de ce genre, à quoi devez-vous réfléchir vous-même? [Sondez : à ce que vous ressentez, désirez et voulez dire.]

- Quels seraient quelques conseils pour une communication réussie?

- Comment dire «non » de manière respectueuse?

- Quels sont les droits de chacun? [Sondez : le droit d'exprimer son opinion, le droit de dire non, le droit de protéger sa propre santé.]

- À qui revient la responsabilité d'entamer ce genre de conversation dans une relation? Pourquoi ? 


\section{le sida : apprendre des autres}

APERCQU : Les élèves écoutent le récit d'une personne séropositive.

OBJECTIFS : Aider les élèves à comprendre et à développer de la compassion pour les personnes vivant avec le VIH et le sida.

\section{DURÉE :}

Étape 1: 10 minutes (avant le jour de l'activité en soi)

Étapes 2-4 : 40 minutes

\section{MATÉRIEL :}

Tableau noir + craie ; feuille à distribuer (en l'absence d'invité).

\section{PRÉPARATION :}

Invitez une ou deux personnes séropositives (idéalement un homme et une femme). Si cela n'est pas possible, invitez un représentant d'un groupe de lutte contre le VIH et le sida, ou un accompagnant de personnes vivant avec le VIH à parler de son expérience personnelle. Informez votre invité de l'âge de vos élèves, de ce qu'ils savent du $\mathrm{VIH}$ et du sida et du temps dont vous disposez pour la présentation et les questions. Demandez peutêtre à parler des sujets suivants :

Découvrir son état VIH

Révéler son état VIH

Opprobre et discrimination

Obtenir de l'aide

Soins de santé et traitement

Vie quotidienne

Vivre avec le VIH : différences entre les hommes et les femmes

Procédez à l'étape 1 avant la visite.

\section{INSTRUCTIONS}

\section{Avant la visite de l'invité}

1 Informez les élèves que, lors de la prochaine session, un ou plusieurs invités viendront parler de leur expérience du VIH. Demandez : De quoi aimeriezvous qu'ils vous parlent? Quelles sont vos craintes? Répondez à celles exprimées, en révisant au besoin l'information sur le VIH. Rappelez aux élèves les règles de base applicables; au besoin, invitez-les à formuler les règles de comportement approprié à l'égard d'un invité.

Autre activité possible : S’il ne vous est pas possible d'obtenir la participation d'un invité, référez-vous à la feuille d'histoires vraies proposée ici. Invitez les élèves à donner des noms locaux aux personnages et à lire les récits à voix haute. Invitez-les aussi à poser leurs questions avant de passer aux étapes 3 et 4 .

\section{Au moment de la visite}

2 Présentez brièvement et remerciez votre invité. Indiquez la durée de la présentation.

3 Réservez un peu de temps pour que les élèves puissent poser leurs questions, puis remerciez encore l'invité.

Juste après le départ de l'invité

4 Animez la discussion en posant ces questions :

- Quels ont été vos sentiments ou impressions pendant la présentation?

- Quelles parties de la présentation vous ont paru les plus utiles ou les plus surprenantes?

- Qu'avez-vous appris sur ce que représente le VIH ?

- Quels sont les sentiments révélés par l'invité?

- Quels mythes ou stéréotypes a-t-il rencontrés?

- Avez-vous encore des questions?

- Quelle est la chose la plus importante que vous avez apprise ou vécue aujourd'hui ? Quelle différence cela va-t-il faire pour vous? [Sondez : Je me sentirai et me comporterai différemment à l'égard des personnes vivant avec le VIH et le sida. Je ferai plus attention à la prévention du VIH.] 


\section{histoires vraies (à utiliser en l'absence d'invité)}

MWENZI : Je suis zambienne, j'ai 22 ans et je suis séropositive à VIH, le virus qui cause le sida. Quand j'étais petite, mes parents sont tous les deux morts du sida. J'ai été élevée principalement par ma sœur aînée. À 19 ans, je me suis retrouvée enceinte et je suis allée au dispensaire, où on m'a fait un test VIH. Quand on m’a dit que j'étais séropositive, je n’arrivais pas à le croire. Je me sentais et j'avais l'air en bonne santé. Je ne voulais pas mourir. Quand j'ai annoncé la nouvelle à mon copain, il a d'abord ri : "Allons, tu n'as pas l'air malade. Ils essaient de te faire peur. » Mais son test l'a révélé séropositif aussi. Heureusement, ma sœur est très aimante. Elle m'aide à payer mes médicaments et me soutient de bien d'autres façons. J'ai décidé de ne pas laisser le VIH devenir un obstacle total à ma vie. Si tel est mon choix, je peux me battre. Heureusement que j’ai été testée. Ça m’a permis de commencer le traitement antirétroviral qui me garde en bonne santé et qui a protégé mon enfant contre le virus du sida. Le plus dur, c'est quand les gens me maltraitent à cause de mon état. J'ignore combien de temps je vais rester en bonne santé mais je vis pleinement ma vie. Et j'ai un travail : j'explique à d'autres jeunes comment se protéger, eux-mêmes et leurs partenaires, de cette maladie.
BRETT : À 18 ans, j'étais crâneur, les cheveux à la punk. Je suivais mes cours le jour et faisais les clubs la nuit. Mais deux ans plus tard, j'apprenais que j'avais contracté le virus qui cause le sida. Je n'en revenais pas. Ma santé me lâchait, mais j’avais ma jeunesse. J'étais plein de cran et idéaliste. Je croyais que nous — patients, médecins, scientifiques et chercheurs si nous en avions vraiment l'intention, nous allions guérir le sida. Je sais que ça paraît idéaliste, mais nous avions alors besoin de cette inspiration. L'idéalisme est ce qui m'a porté quand il y avait peu d'espoir. J'avais toujours voulu écrire. Après avoir reçu mon diagnostic, j'ai su exactement ce sur quoi j'allais écrire et je m’y suis plongé corps et âme. J'ai grappillé juste de quoi lancer un magazine, que j’ai intitulé Plus Voice. Ma vision était celle d'un magazine de vie pour les personnes atteintes du virus, le premier en son genre. Je me sens parfois épuisé, mais mon travail et mes amis me soutiennent. J'ai vécu toute ma vie adulte avec le VIH. Bien sûr, nous n'avons toujours pas de cure au sida, mais en 2008, nous avons d'excellents médicaments, qui font échec au virus. Et qui le font mieux chaque année. Mais ce n'est pas une sinécure. Et pas question d'adopter d'autres comportements malsains. Alors, mettez votre ceinture de sécurité. $\mathrm{Ne}$ fumez pas. Buvez avec modération. Obtenez l'aide psychologique dont vous avez besoin. Restez actif. Utilisez toujours le préservatif — toujours. Et soyez rigoureusement honnêtes avec vous-mêmes, concernant tous les aspects de votre vie. [Adapté avec l'autorisation de Test Positive Aware Network <www.tpan.com>.] 


\section{que faut-il dire? que faut-il savoir?}

APERÇU : Les élèves discutent la responsabilité de divulguer son état VIH à un(e) partenaire sexuel(le). Ils examinent l'équilibre complexe entre le droit à la vie privée et le droit de se protéger contre toute menace. Ils explorent leurs sentiments à l'égard de ce dilemme par composition créative. (Remarque: Avant cette activité, les élèves doivent avoir été minimalement informés sur les droits humains, le consentement, le VIH et le sida.)

\section{DURÉE :}

Étapes 1-5: 45 minutes

Étapes 5-6: 45 minutes

\section{MATÉRIEL :}

Tableau noir + craie

\section{PRÉPARATION :}

Préparez des « questions ouvertes $\gg$ (autres que de type « oui/non ») à poser pour approfondir l'étape 1. Si possible, renseignez-vous sur la prévalence du VIH dans votre contexte. Cette activité peut éveiller d'intenses sentiments chez certains élèves. Réfléchissez à la manière dont vous pouvez les aider. Respectez la vie privée de chacun et n'obligez personne à lire sa composition à voix haute.

OBJECTIFS : Aider les élèves à réfléchir de manière critique à la responsabilité ou obligation qui incombe à chacun de divulguer son état VIH à ses partenaires sexuels.

\section{INSTRUCTIONS}

1 Présentez l'activité en vous inspirant des questions suivantes. [Écrivez les réponses au tableau.]

- Nous allons parler aujourd'hui d'un sujet complexe et délicat : celui de l'obligation ou non de divulguer son état VIH à un partenaire sexuel.

- Pensez-vous que les personnes qui se savent séropositives divulguent toujours leur état?

- Pour quelles raisons une personne peut-elle choisir de ne pas dire son état à son partenaire? [Sondez: pour protéger sa vie privée; peur de l'opprobre, de l'abandon et de la discrimination.]

- Pourquoi ne fait-on pas toujours l'effort de se faire tester? [Sondez: peur d'être séropositif (d'avoir le VIH); gêne à l'idée de se rendre dans une clinique de dépistage; ignorance des centres de dépistage gratuit; bonne santé apparente et incrédulité à l'idée d'être infecté; heures d'ouverture incompatibles avec les heures d'école ou de travail; méfiance que les résultats ne soient pas vraiment privés/secrets.]
- Pour quelles raisons est-il utile de se faire tester? [Sondez: soulagement si le test est négatif, début de traitement s'il est positif; protection des partenaires ultérieurs; information des partenaires actuels et passés pour qu'ils puissent se faire tester aussi.]

2 Invitez les élèves à prendre leur cahier. Expliquez:

- Nous avons réfléchi à quelques raisons pour lesquelles certaines personnes choisissent de ne pas se faire tester ou de ne pas révéler leur état VIH à leurs partenaires. Votre tâche est d'approfondir la question dans un bref récit. Votre histoire ne doit pas dépasser trois pages.

- Vous pouvez raconter l'histoire d'une personne qui choisit de ne pas se faire tester. Ou de quelqu'un qui a attrapé le VIH d'un partenaire qui ne lui avait pas révélé son état séropositif. Ou bien encore de quelqu'un qui se sait infecté et qui le dit à son ou sa partenaire.

- Quels problèmes et sentiments votre récit doitil explorer? Introduisez-y une certaine tension. 
Montrez comment votre personnage en est arrivé à sa décision, ce qu'il ou elle en pense et ce qui s'est passé (comment la décision a affecté la vie du personnage et celle des autres). Réfléchissez aux sentiments du personnage (s'agit-il de peur, soutien, solitude, soulagement, regret, sentiment d'être aimé, appréciation, amertume ?)

- L'histoire peut être factuelle ou fictive. Inventez-la de toutes pièces ou inspirez-vous de votre propre expérience ou de celle de quelqu'un que vous connaissez. Veillez cependant à ne pas utiliser de vrais noms. Et enfin: Votre récit ne doit pas être long, mais il doit être réfléchi. Il s'agit d'une tâche sérieuse, où l'humour n'a pas vraiment sa place. Si vous n'avez pas le temps d'achever votre composition en classe, achevez-la comme devoir.

3 Invitez les élèves à réfléchir et commencer à rédiger leur composition. Indiquez-leur si vous allez leur demander de lire leur récit. Invitez-les aussi à vous consulter s'ils ont du mal à définir leur sujet. [Suggérez: Un garçon a peur de se faire tester parce que ses deux parents sont morts du sida; une fille a peur de dire à son partenaire qu'elle est séropositive à cause d'un viol, parce que son petit ami pense qu'elle était vierge avant de le rencontrer.]

4 Ramassez les copies, ou dites aux élèves de les achever comme devoir. Veillez cependant à lire vous-même chaque récit avant la session éventuelle de lecture devant la classe pour vous assurer qu'ils soient tous appropriés et anonymes.
5 Invitez les élèves à lire leur composition. (Tous les récits ne doivent pas être lus.)

6 Discutez ces questions pour conclure :

- Percevez-vous le dilemme autrement après avoir entendu le récit d'un camarade ou avoir rédigé le vôtre ? En quoi pensez-vous que vos attitudes ou vos sentiments ont changé? [Sondez: Vous parait-il maintenant plus facile ou plus difficile de se faire tester que vous ne le pensiez? Est-il plus ou moins important de divulguer son état que vous ne le pensiez? Réagiriez-vous différemment à un partenaire amoureux ou sexuel qui vous avoue être séropositif?]

- La question de divulguer son état VIH est-elle toujours claire et nette?

- Comment s'assurer d'être aussi honnête que possible envers soi-même et ses partenaires pour protéger la santé de tous?

- Faut-il proposer ou promouvoir un dépistage et conseil facultatif anonyme à chaque visite clinique ou vaut-il mieux que les prestataires n'effectuent le test que sur demande spécifique?

- Que dire en conclusion? [Essayez de conclure la session sur un commentaire relatif à la pandémie du sida qui appelle à l'honnêteté, à la compréhension, à la discipline personnelle, au courage et à l'optimisme.] 


\section{connaissance de la contraception}

\section{DURÉE :}

45 minutes

\section{MATÉRIEL :}

Tableau noir + craie; copies de la grille de mots croisés et de la « liste de méthodes contraceptives $»$; bandelettes de papier portant le nom de méthodes contraceptives; sac ; 4 copies de la fiche d'information sur les méthodes contraceptives (GUIDE compagnon de ce volume) ou autre texte fiable ; si possible, une montre (avec secondes) et petit prix pour l'équipe gagnante.

\section{PRÉPARATION :}

Photocopiez la grille de mots croisés (avec ou sans la clé). Lisez la liste de méthodes contraceptives et supprimez-en celles dont vous ne parlerez pas. Avant la session, informez les élèves sur les méthodes retenues. Écrivez le nom de chacune sur une bandelette de papier. Pliez les bandelettes et mettez-les dans un sac. Procédez en fonction de la remarque de l'étape 3. Rafraîchissez votre connaissance des méthodes.

APERC̣U : Une grille de mots croisés et un jeu aident les élèves à réviser leur connaissance de la contraception. Ils en discutent aussi la responsabilité partagée. (Remarque : Cette activité peut servir d'introduction à la contraception ou de révision.)

OBJECTIFS: Passer en revue et renforcer la connaissance des méthodes contraceptives ; renforcer l'expression écrite.

\section{INSTRUCTIONS}

Devoir (à donner avant cette activité) :

Remplissez la grille de mots croisés sur les méthodes contraceptives. (Distribuez une grille vierge à chacun.)

Passez le lendemain à l'étape 1.

1 Donnez ensemble les solutions à la grille de mots croisés. Répondez aux questions.

2 Expliquez aux élèves qu'ils vont jouer au jeu des " 5 questions », pour tester leur connaissance des différentes méthodes contraceptives. Formez trois équipes et expliquez :

- Ce sac contient des bandelettes de papier, revêtues chacune du nom d'une méthode. L'équipe 1 prend une bandelette dans le sac.

- Les équipes 2 et 3 essaient de deviner la méthode inscrite sur la bandelette de l'équipe 1, en posant des questions auxquelles l'équipe 1 ne peut répondre que par «oui» ou «non ». [Expliquez le type de question à poser.] L'équipe 2 pose la première question.
3 Expliquez les règles. [Remarque: Si les élèves ont déjà une certaine connaissance des méthodes de contraception, utilisez l'approche des questions « ouil non » pour deviner la réponse. S'ils n'en ont pas encore connaissance, il pourra être utile de donner à chaque équipe sa propre fiche d'information.]

- Pour assurer la bonne réponse aux questions posées, l'équipe 1 peut consulter la fiche d'information. [montrez-la]. Mais attention, vous n'avez que 30 secondes pour répondre « oui » ou «non ». Vous pouvez vous consulter rapidement avant de répondre. Vous devrez me montrer votre bandelette pour que je puisse corriger vos erreurs éventuelles. Si vous donnez une information incorrecte sur votre méthode, votre équipe perd $1 / 2$ point. N'oubliez pas de ne répondre que par « oui » ou «non ». Chargez un membre de votre équipe de tenir le compte des questions posées et d'annoncer quand cinq réponses ont été données sans que la méthode ait été correctement identifiée. 
- Après avoir reçu la réponse de l'équipe 1 à sa première question, l'équipe 2 peut essayer de deviner le nom de la méthode contraceptive. Si elle se trompe (ou choisit de ne pas deviner), l'équipe 3 peut poser une question et ainsi de suite, à tour de rôle, entre l'équipe 2 et l'équipe 3 jusqu'à ce que l'une d'entre elle donne la bonne réponse (et gagne un point) ou que cinq questions aient été posées.

- Si, après cinq questions, aucune équipe n’a deviné correctement la méthode, l'équipe 1 annonce la réponse correcte, clarifie l'information importante à savoir au sujet de cette méthode et gagne le point.

4 Expliquez la suite du jeu.

- L'équipe 2 prend ensuite une bandelette et les équipes 1 et 3 lui posent leurs questions, et ainsi de suite jusqu'à la fin de la session ou des bandelettes.

- Comprenez-vous tous bien les règles ? [Si nécessaire, démontrez-les en prenant une bandelette (méthode) dans le sac.]

5 Désignez un chronométreur chargé de faire respecter les 30 secondes admises pour chaque question. Désignez un autre éleve chargé de marquer les points au tableau.
6 Invitez l'équipe 1 à prendre une bandelette et donnez-lui une copie de la fiche d'information. Vérifiez chaque bandelette choisie et gardez vousmême une copie de la fiche pour référence rapide au cas où vous devriez corriger une mauvaise réponse.

7 Après identification correcte de la méthode ou réponse à cinq questions, arrêtez et donnez le point à l'équipe gagnante. Invitez ensuite l'équipe suivante à prendre une bandelette et continuez à jouer pendant environ 20 minutes, ou jusqu'à 10 minutes avant la fin de la session.

8 Déclarez l'équipe gagnante et remettez-lui son prix.

9 Réservez 5 à 10 minutes à la discussion :

- Qu'avez-vous appris aujourd'hui que vous ne saviez pas et qui vous a paru intéressant au sujet d'une méthode contraceptive?

- [La question suivante peut être donnée comme devoir ; voir plus bas.] Tout le monde doit-il être au courant de la contraception ? À qui revient la responsabilité de se protéger contre les grossesses non désirées?

Devoir : Rédigez un paragraphe en réponse à ces questions : Tout le monde doit-il être au courant de la contraception? À qui revient la responsabilité de se protéger contre les grossesses non désirées ? Répondez directement ou sous forme d'histoire. 
mots croisés : méthodes contraceptives

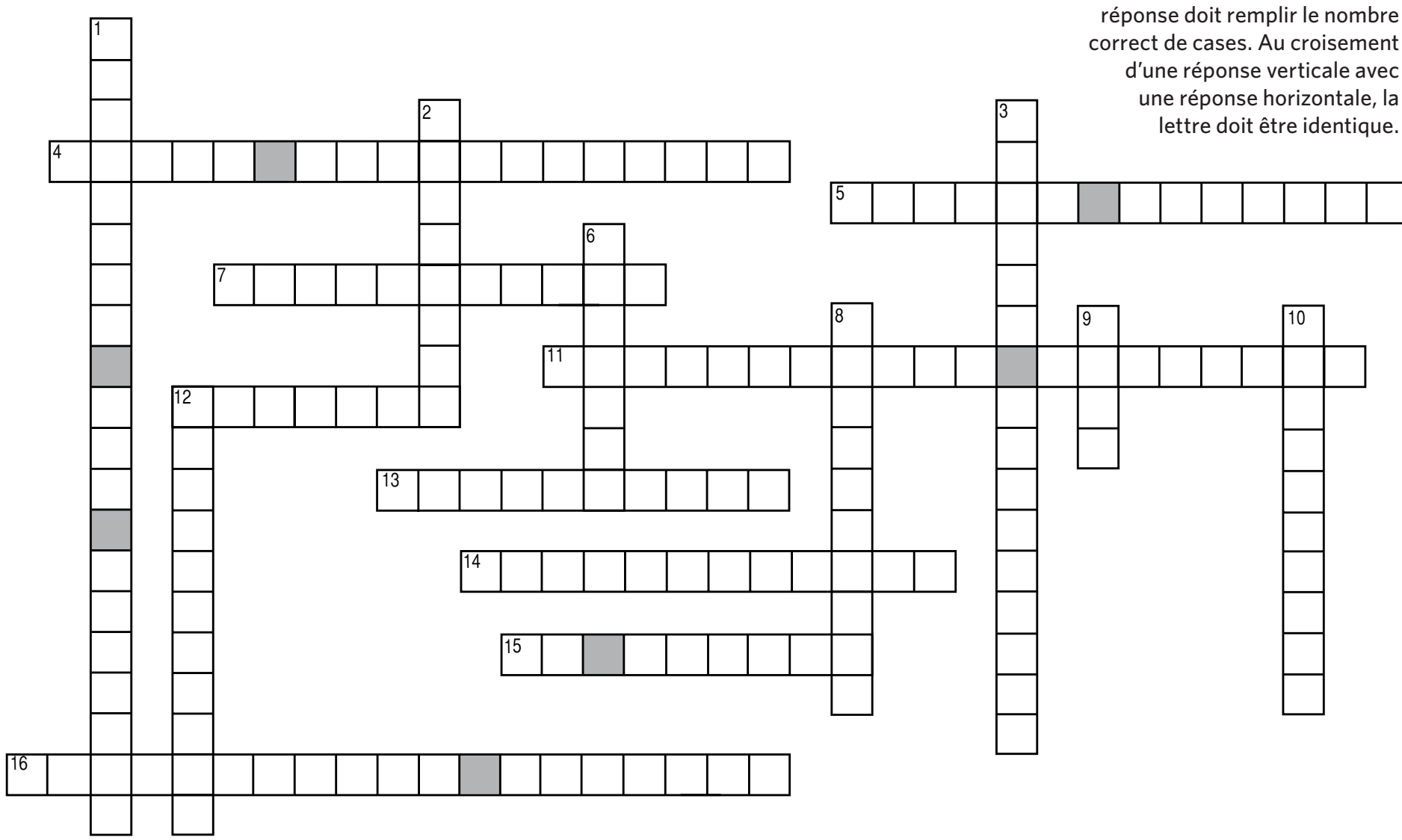

\section{à I'horizontale}

4. Une femme ou une fille l'applique sur sa peau comme un sparadrap; il ne protège pas contre les IST et le VIH ( 2 mots).

5. Dispositif en forme de cercle que la femme ou la fille s'introduit dans le vagin ; il ne protège pas contre les IST et le VIH (2 mots).

7. Substances diverses introduites dans le vagin pour tuer le sperme.

11. Capuchon dont l'homme ou le garçon recouvre son pénis pendant les rapports sexuels ; évite la grossesse et protège contre les IST et le VIH (2 mots).

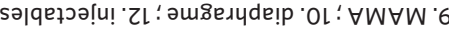

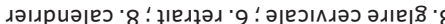

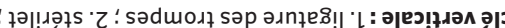

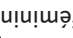

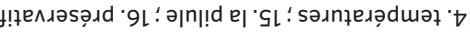

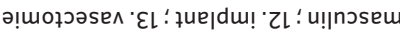

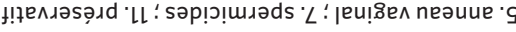

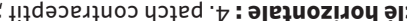

12. Petit bâtonnet introduit dans le bras d'une femme ou d'une fille.

13. Procédure chirurgicale qui empêche l'émission de spermatozoïdes.

14. Par la méthode des_, la femme ou la fille détermine ses jours féconds à l'aide d'un thermomètre.

15. Une femme ou une fille la prend chaque jour pour éviter de se retrouver enceinte (appellation courante, 2 mots).

16. Fine poche que la femme ou la fille s'introduit dans le vagin pour empêcher le sperme de pénétrer son corps ( 2 mots)
Lisez chaque définition et remplissez la grille. Votre éponse horizontale, la

\section{à la verticale}

1. Opération consistant à couper ou ligaturer les trompes de Fallope d'une femme pour empêcher l'ovule et le sperme de se rencontrer (3 mots).

2. Dispositif, souvent en forme de $\mathrm{T}$, introduit dans l'utérus.

3. Une femme ou une fille peut identifier le moment où elle est féconde en fonction de la quantité et de la consistance de sa (2 mots).

6. Méthode consistant à retirer le pénis du vagin avant l'éjaculation.

8. Une femme ou une fille peut calculer le moment où elle est féconde en suivant ses cycles menstruels sur un

9. Méthode naturelle liée à l'allaitement maternel (abréviation)

10. Dôme en caoutchouc qu'on remplit de spermicide et qu'on introduit dans le vagin pour recouvrir le col de l'utérus.

11. Piqûres données régulièrement à la femme ou à la fille pour empêcher l'ovulation et épaissir la glaire cervicale (pluriel). 


\section{liste de méthodes contraceptives utilisées dans le monde}

Méthodes temporaires contrôlées par l'utilisateur (pour empêcher le sperme d'atteindre l'ovule)

Préservatif masculin

Préservatif féminin

Diaphragme

Cape cervicale

Spermicides

\section{Méthodes internes au corps}

Courte durée, sous contrôle de l'utilisatrice

Contraception orale (pilule)

Contraception d'urgence

Longue durée

Injectables (Depo-provera)

Anneau vaginal hormonal

Patch hormonal

Implant hormonal

Stérilet

Méthodes naturelles (requérant des comportements spécifiques et une bonne compréhension de son corps) Aménorrhée lactationnelle (MAMA)

Retrait

Méthode de conscience de la fécondité - glaire cervicale

Méthode de conscience de la fécondité - température

Méthode de conscience de la fécondité - calendrier, jours fixes ou collier (parfois appelée méthode du rythme ou Ogino)

Abstinence

Jeux sexuels sans pénétration

Méthodes chirurgicales permanentes

Ligature des trompes (ou autres méthodes de stérilisation féminine)

Vasectomie 


\section{se mettre à sa place : la décision d'interrompre une grossesse}

APERCWU : Les élèves lisent quelques études de cas et discutent les raisons pour lesquelles les femmes se font avorter.

OBJECTIFS: Amener les élèves à discuter trois raisons pour lesquelles les femmes et les filles choisissent l'avortement; renforcer la réflexion analytique et l'aptitude au dialogue.

\section{DURÉE :}

60 minutes (l'activité peut être divisée en deux sessions)

\section{MATÉRIEL :}

Tableau noir + craie ; copie d'une étude de " cas d'avortement 》 par groupe (ou une copie de tous les cas avec attribution d'un cas par groupe); le volume GUIDE de Un seul programme (sur $<w w w . u n s e u l p r o g r a m m e . o r g>$ ) ou une photocopie de la section sur la grossesse non planifiée et l'avortement (unité 7).

\section{PRÉPARATION :}

Lisez dans le materiel

recommandé la liste des raisons pour lesquelles les femmes choisissent d'avorter. Lisez et adaptez, au besoin, les études de cas à votre culture. Veillez à ce que les cas reflètent différentes raisons de choisir l'avortement. Familiarisez-vous avec vos lois locales sur l'IVG (concernant les jeunes surtout) et l'octroi de pensions alimentaires.

\section{INSTRUCTIONS}

1 Commencez par les questions suivantes:

- Nous allons parler aujourd'hui des décisions compliquées que l'on prend dans la vie. Pensez à un moment où vous - ou une personne proche de vous - avez dû prendre une décision difficile, inacceptable pour certains. [Laissez réfléchir.]

- Qu'avez-vous alors ressenti ? Avez-vous (ou la personne à qui vous pensez) été soutenus ?

- Si non, comment cela a-t-il affecté la décision, et ce que vous avez ressenti ?

- Pour des millions de femmes et de filles, se retrouver enceintes sans l'avoir planifié devient un moment décisif. Pour certaines, la décision est simple et directe; pour d'autres, elle est difficile et complexe.

- Nous allons parler aujourd'hui de la décision de se faire avorter. Nous n'allons pas discuter du bien ou du mal de l'avortement, mais plutôt de ce qui entre en jeu dans cette décision, pour essayer de comprendre pourquoi certaines filles et femmes choisissent l'avortement.
- Quels sont les choix possibles pour une fille ou une femme enceinte? [Sondez: avoir l'enfant et l'élever; avoir l'enfant et le faire adopter; ou interrompre la grossesse.]

2 Répartissez les élèves en petits groupes de quatre ou cinq. Distribuez à chaque groupe une étude de cas à lire, en donnant un nom à la personne du récit, puis discutez [écrivez les questions au tableau] :

- Pourquoi cette fille s'est-elle fait avorter?

- Quel rôle ont joué les autres dans sa décision?

[Pendant que les élèves travaillent, écrivez au tableau: Raisons pour lesquelles les filles et les femmes choisissent l'avortement.]

3 Rassemblez la classe au complet. Invitez le premier groupe à lire son cas et animez 5 à 7 minutes de discussion :

- Pourquoi cette fille a-t-elle choisi l'avortement ?

- Pensez-vous tous que telles étaient ses raisons? En voyez-vous d'autres? [Sondez pour obtenir d'autres raisons pertinentes au cas.] 
- Quel rôle ont joué les autres (partenaire, famille, amis ou autres) dans sa décision, directement ou dans son esprit?

- Y a-t-il d'autres commentaires?

4 Répétez l'approche pour chaque cas. Prévoyez 7 minutes par cas.

5 Réservez 10-15 minutes à ces questions :

- À votre avis, ces raisons (au tableau) expliquentelles pourquoi les jeunes femmes se font avorter? Pensez-vous qu'il y en a d'autres? [Ajoutez les autres raisons données à la liste.]

- À l'échelle mondiale, la majorité des femmes qui se font avorter sont mariées. Dans quelles situations pensez-vous qu'une femme mariée puisse recourir à l'avortement?

- Dans certains pays, l'avortement est admis dans de nombreuses circonstances; dans d'autres, il est largement interdit par la loi (ou n'est admis que dans certaines circonstances).

- Pourquoi les femmes et les filles se font-elles avorter même quand la procédure est illégale et potentiellement dangereuse?
Devoir : Sélectionnez une citation ci-dessous.

Juger quelqu'un, ce n'est pas le définir, c'est se définir soi-même.

— Wayne Dyer

On ne peut jamais juger la vie d'une autre personne, car elle seule sait ce qu'elle a souffert et ce à quoi elle a renoncé. C'est une chose que de penser qu'on est sur le droit chemin, c'en est une autre de penser qu'il n'y a qu'un chemin.

$$
\text { — Paulo Coelho }
$$

N'oubliez pas que je suis humain. Avant de me juger et de décider comment me traiter, faites quelques pas à ma place. Sans doute comprendrez-vous mieux qu'en suivant la voie du milieu, nous pourrons faire le reste $d u$ chemin ensemble.

$$
\text { — Eric Harvey et Steve Ventura }
$$

J'aimerais donner satisfaction à tous, mais ce faisant, je finirais peut-être par ne donner satisfaction à personne. J'en suis arrivé à la conclusion que la meilleure solution est de donner satisfaction à sa propre conscience et de laisser le monde arrêter son propre jugement, favorable ou non.

$$
\text { — Mohandas K. Gandhi }
$$

Copiez la citation de votre choix et répondez aux questions suivantes :

- Que pensez-vous de cette citation?

- Êtes-vous d'accord ou non avec l'auteur?

- Quel est le rapport entre le sens de cette citation et la situation d'une fille qui choisit d'interrompre une grossesse non désirée? 


\section{cas d'avortement}

\section{GROUPE 1}

Je m'appelle . Nous avons tous les deux 22 ans, mon copain $\mathrm{Lu}$ et moi, et nous sortons ensemble depuis deux ans. Je prends la pilule mais je l'ai oubliée une fois ou deux le mois passé et je me suis retrouvée enceinte. Je viens de commencer un nouveau boulot que j'adore mais qui ne paie pas encore bien. J'aime ma vie comme elle est et je ne veux pas d'enfant. Je suis allée dans une clinique de planning familial où l'infirmière m'a fortement conseillé de me marier et d'avoir l'enfant. Lu est d'accord avec elle parce qu'il pense que je voudrai être mère un jour, alors, pourquoi pas maintenant? Ça m'énerve, cette pression d'avoir un enfant dont je ne veux pas. J'ai décidé d'aller voir un médecin qui accepte d'interrompre les grossesses sans poser trop de questions.

\section{GROUPE 2}

Je m'appelle J'ai 20 ans et je suis la première personne de mon village à avoir été acceptée à l'université, dans la capitale. Ma famille, mes amis et mes voisins attendent beaucoup de moi. Pour eux, ma réussite marquera le début d'un véritable changement dans la communauté. Peu après le début des cours, j’ai commencé à sortir avec un autre étudiant. Nous avons commencé à avoir des rapports sexuels après quelques mois. Nous utilisions généralement le préservatif, mais pas toujours dans la passion du moment. Quand je me suis retrouvée enceinte, j'ai voulu en parler à mon copain pour prendre une décision ensemble, mais il s'est aussitôt montré distant et non disponible. Un ami commun m'a dit qu'il pensait que j'essayais de le piéger et de le forcer à m'épouser. Je n'ai même pas envie de me marier, mais je ne veux pas non plus être mère célibataire à mon âge. Je veux terminer mes études et avoir l'occasion de réaliser mes rêves. J'aurais aimé avoir le soutien de ma famille mais j'ai eu peur de les décevoir et c'est bien la dernière chose que je voudrais faire. J’ai donc décidé de ne rien dire à personne et d'utiliser mon allocation de séjour pour me faire avorter. 


\section{cas d'avortement}

\section{GROUPE 3}

Je m'appelle et j'ai 17 ans. Quand j'ai appris que ma petite amie était enceinte, j’ai pensé : «Quoi ? Ouah! Oh non!» Mes sentiments étaient un mélange de choc, de peur, d'inquiétude et d'étonnement. J'étais même fier, dans un sens, de savoir que je n'étais pas stérile. Mais en fin de compte, il fallait résoudre la question de savoir ce que nous allions faire : parents, adoption, IVG ? Ma copine et moi sommes encore à l'école et nous savons que nous sommes trop jeunes pour être de bons parents. Nous avons décidé que la meilleure solution pour nous était l'avortement. Un ami nous a conseillé une clinique et nous y sommes allés ensemble. Quelqu'un nous a expliqué exactement ce qui allait se passer. Avant de nous laisser partir, on nous a aussi parlé de la contraception et on nous a donné une boîte de préservatifs. J'ai dû faire face à beaucoup de mes propres émotions, mais je suis fier d'avoir aidé ma copine dans un moment difficile.

\section{GROUPE 4}

Je m’appelle . J'ai 15 ans et je vis avec ma famille étendue. Chaque année, mon oncle et ma tante nous rendent visite avec leur fils, mon cousin, qui a maintenant 18 ans. Un jour, cette année, pendant que tout le monde était sorti, il m'a demandé s'il pouvait me toucher et a voulu que je lui fasse la même chose. Ça m’a paru étrange et ça ne m’a pas vraiment plu mais il est mon cousin aîné et je ne voulais pas le vexer. Quand il s'est mis à me déshabiller et à se coucher sur moi, j’ai eu peur et j'ai essayé de le repousser mais il était trop fort et il m’a violée. Quand j'ai découvert que j'étais enceinte, j'ai de nouveau eu peur. Je voulais demander l'aide de ma mère mais j'avais trop de honte pour lui dire ce qui s'était passé. J'ai fini par trouver le courage de le lui dire. Elle m'a immédiatement fait avorter et a refusé catégoriquement de parler de la situation avec moi. J'étais soulagée de ne plus être enceinte, mais j'aurais voulu pouvoir parler à quelqu'un de cette expérience pénible. 


\section{l'inspiration du changement}

APERCQU : Les élèves choisissent une citation qui les inspire et en explorent le sens et I'application dans leur propre vie.

OBJECTIFS: Accroître la motivation et la confiance des élèves : ils peuvent changer le monde dans lequel ils vivent.

\section{DURÉE :}

60-90 minutes, suivant le nombre de citations sélectionnées.

\section{MATÉRIEL :}

Tableau noir + craie ; 2 pages « citations sur la justice sociale et l'activisme».

\section{PRÉPARATION :}

Lisez les citations et éliminez celles qui ne se prêtent pas à votre contexte. Ajoutezen éventuellement d'autres d'auteurs locaux. Ajustez le nombre de citations (et de présentations) au temps dont vous disposez. Photocopiez les citations que vous allez utiliser ou écrivez-les au tableau.

\section{INSTRUCTIONS}

1 Expliquez:

- Nous allons explorer aujourd'hui ce que c'est que d'œuvrer pour la justice sociale ou le changement, même minime. Nous allons analyser quelques citations, de personnalités qui ont, à travers leurs efforts, fait une différence.

- Lisez la liste et choisissez la citation qui vous inspire le plus. Prenez ensuite vos cahiers et copiez votre citation en haut d'une page (avec le nom de la personne à qui elle est attribuée).

- Réfléchissez au sens de votre citation. Rédigez-y une réponse d'au moins trois paragraphes. Dans le premier, expliquez ce que vous pensez être le message de la citation. Dans le deuxième, indiquez le sens qu'elle représente pour vous et pourquoi vous l'avez sélectionnée. Dans le troisième, décrivez ce que son application représenterait dans votre propre vie (ou, si vous l'appliquez déjà, parlez-en). [Donnez aux élèves le temps d'écrire.]
2 Demandez si quelqu'un a choisi la première citation. Si oui, invitez l'élève à lire sa composition. (Donnez la permission de ne pas lire les sections que les élèves désirent garder privées.) Si plusieurs élèves ont choisi une même citation, donnez-leur peut-être le choix de lire leurs paragraphes. Procédez de même pour chaque citation. Prévoyez environ 12 minutes de lecture.

3 Pour conclure, posez ces questions-guides :

- Quels grands messages tirez-vous de ces citations?

- Quelles sont les récompenses personnelles dont jouissent les militants pour la justice sociale?

- Quels sont les défis et les risques?

- Est-il important que les citoyens ordinaires militent pour les causes qui leur sont chères?

Devoir : Recherchez un moyen de partager votre citation : parlez-en aux membres de votre famille, écrivez-la et affichez-la quelque part, ajoutez-la à votre signature électronique ou trouvez une autre façon d'en communiquer l'inspiration. Rédigez votre approche pour demain. 


\section{citations sur la justice sociale et l'activisme}

Soyez le changement que vous voulez voir dans le monde.

- Mohandas Gandhi, « père de la nation indienne ». Gandhi est à l'origine de la désobéissance civile pacifique, qui a mené à l'indépendance de l'Inde de la tutelle britannique et inspiré les mouvements pour les droits civils et la liberté partout dans le monde.

Se trouver dans un contexte d'oppression et s'en laver les mains revient à renforcer la puissance de l'oppresseur, à se ranger de son côté.

- Paulo Freire, célèbre pédagogue brésilien, pionnier des pratiques pédagogiques de la conscientisation des opprimés.

Le pouvoir ne concède rien sans exigence revendicatrice. Jamais il n'a donné et jamais il ne donnera. Le degré de soumission admis marque la mesure exacte de l'injustice et du mal qui sont imposés et il en sera ainsi tant que les mots, les coups ou les deux n'y résisteront pas. Les limites des tyrans sont dictées par l'endurance de ceux qu'ils oppriment.

— Frederick Douglass né esclave, est devenu l'un des protagonistes du mouvement contre l'esclavage aux ÉtatsUnis. Auteur, orateur et conseiller du président Abraham Lincoln, il croyait en l'égalité universelle.

La liberté n'est pas un don qu'un peuple confère à un autre. Chacun la revendique comme sienne et personne ne peut la lui refuser.

- Kwame Nkrumah, fondateur et premier président du Ghana moderne et père du panafricanisme.

[La] responsabilité ne revient pas uniquement aux dirigeants de nos pays ou à ceux que nous avons désignés ou élus pour assumer telle ou telle fonction. Elle revient à chacun de nous, individuellement.

- Sa Sainteté le 14 ' Dalaï-Lama, chef spirituel du bouddhisme tibétain et activiste pour l'autonomie du Tibet.

Ne doutez jamais qu'un petit nombre de gens décidés puisse changer le monde. C'est en fait toujours ainsi que le monde a changé.

- Margaret Mead, anthropologue américaine. 


\section{citations sur la justice sociale et l'activisme}

Le monde est dangereux à vivre! Non pas tant à cause de ceux qui font le mal, mais à cause de ceux qui regardent et laissent faire.

- Albert Einstein, Lauréat du prix Nobel de physique principalement connu pour sa théorie de la relativité. Fervent opposant du nazisme, il a aussi milité contre les essais nucléaires et le racisme.

Quand les nazis sont venus chercher les communistes, je n'ai rien dit; je n'étais pas communiste. Quand ils ont enfermé les sociaux-démocrates, je n'ai rien dit; je n'étais pas social-démocrate. Quand ils sont venus chercher les syndicalistes, je n'ai pas protesté; je n'étais pas syndicaliste. Quand ils sont venus chercher les juifs, je n'ai rien dit; je n'étais pas juif. Et quand ils sont venus me chercher, il ne restait personne pour protester.

- Martin Niemöller, célèbre pasteur et théologien allemand antinazi ayant survécu aux camps de concentration et consacré le reste de sa vie au pacifisme.

L'injustice quelque part est une menace à la justice partout. Nous sommes tous pris dans un réseau inextricable de mutualité, tissés dans la même toile du destin. Ce qui affecte directement les uns affecte indirectement tous les autres.

- Martin Luther King, Jr., pasteur américain, leader le plus célèbre du mouvement pour les droits civils aux ÉtatsUnis. Défenseur de la non-violence et activiste pour le traitement égal de toutes les races, il aura été le plus jeune lauréat du prix Nobel de la paix.

Ce sont les petites choses qui font la différence. Moi, je plante des arbres.

- Wangari Maathai, militante écologiste, féministe et politique kenyane. Fondatrice du Green Belt Movement et lauréate du Prix Nobel de la Paix 2004 (première femme africaine à avoir reçu le prix). Elle a aussi été élue au parlement kenyan. 


\section{une cause qui me tient à cœur}

APERCQU : Les élèves identifient une cause ou un problème social qui leur tient à cœur. Ils explorent l'idée du militantisme dans leur propre vie.

OBJECTIFS : Aider les élèves à considérer et identifier une cause qui leur est chère ; à pratiquer leurs aptitudes à la communication et à l'expression en public ; à réfléchir à un changement positif qu'ils pourraient apporter dans leur propre vie.

\section{INSTRUCTIONS}

1 Présentez le sujet:

- Nous avons parlé de différentes questions qui affectent nos vies - en ce qui concerne le genre, la santé et les droits surtout. Quels sujets spécifiques avons-nous étudiés? [Sondez: sujets couverts.]

- Chacun d'entre vous va maintenant identifier un problème sociétal qui lui tient à cœur, dans les domaines dont nous avons parlé.

2 Distribuez la liste « Un problème qui me tient à cœur» et expliquez :

- Écrivez votre nom sur les deux pages, puis lisez la liste de problèmes de justice sociale en pensant à ceux qui vous tiennent à cœur.

- La liste est divisée en trois catégories (genre, santé sexuelle et droits sexuels). Cochez trois points qui vous intéressent. Ils peuvent tous être dans la même catégorie ou dans des catégories différentes.

- Vous pouvez aussi ajouter une question non incluse que vous jugez importante.
3 Groupez les élèves par couples de même sexe et expliquez :

- Deux par deux, vous allez discuter pendant 20 minutes d'une ou deux questions qui vous tiennent à cœur.

- Partagez ce qui vous paraît important dans vos choix ou pourquoi vous désirez en apprendre davantage sur la question. Peut-être avez-vous une histoire à raconter.

- Écoutez-vous l'un ou l'une l'autre avec attention et engagez un dialogue respectueux.

- N'oubliez pas de traiter vos camarades de classe avec respect. La justice et le respect en toute égalité commencent ici, dans cette classe, dans la manière dont nous nous traitons les uns les autres.

4 Rassemblez le groupe au complet et demandez si quelqu'un désire dire aux autres le ou les problèmes qu'il ou elle a choisis et pourquoi. Laissez parler tous les volontaires.

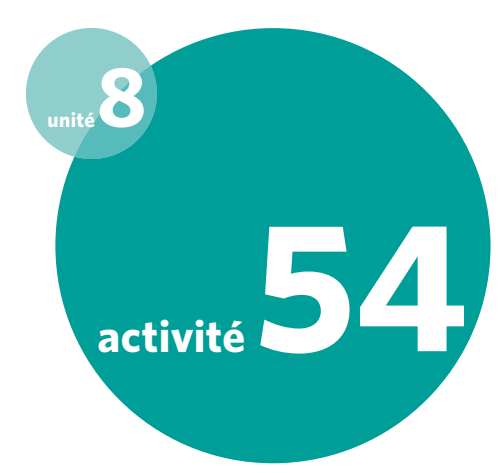

\section{DURÉE :}

Étapes 1-4: 45 minutes

Étapes 5-8: 45 minutes

\section{MATÉRIEL :}

Tableau noir + craie ; une copie des feuilles « une cause qui me tient à cœur » et

«lettre du fond du cœur » par élève; une copie de l'unité 8 du GUIDE compagnon de ce volume, accessible sur: $<w w w$. unseulprogramme.org $>$.

\section{PRÉPARATION :}

Lisez la liste « une cause qui me tient à cœur » et supprimez-en les points qui feraient courir des risques à vos élèves. Ajoutez-en d'autres localement pertinents. Lisez la feuille « lettre du fond du cœur ». Si vous prévoyez la réalisation

d'un projet d'activisme (selon I'unité 8 du GUIDE), gardez la colonne de droite sur la page « lettre du fond du cœur ». Supprimez sinon cette colonne pour éviter que les élèves ne se lancent dans une action militante sans guide ni soutien. 
5 Présentez aux élèves le concept de l'action pour le changement social (activisme ou militantisme) :

- Certaines personnes s'engagent dans de vastes campagnes pour changer le monde. Avez-vous entendu parler d'actions pour la résolution d'un problème de notre liste qui vous tient à cœur ?

- Et concernant les sujets dont nous avons parlé ici : l'égalité de genre et la santé et les droits sexuels et génésiques? [Prenez quelques exemples. Au besoin, citez un exemple de l'unité $8 d u$ GUIDE ou de votre propre expérience.]

- Quel serait un exemple de changement que de telles actions ont apporté dans le monde? [Par exemple: arrêt de la mutilation génitale des filles, nombres croissants de filles à l'école, adhésion des hommes aux campagnes contre la violence sexiste, légalisation de l'avortement, écoles plus sûres pour les jeunes homosexuels et autorisation des adolescentes enceintes à rester à l'école.]

6 Introduisez l'idée du changement de petite envergure et menez la discussion sur les points suivants :

- Le militantisme vise souvent de grands changements mais même un petit effort peut susciter un changement utile - dans votre propre vie ou autour de vous.

- Imaginez et considérez une petite mesure que vous pourriez prendre pour faire la différence, spécifiquement, en faveur de l'égalité de genre ou pour protéger vos droits sexuels et génésiques ou ceux d'une autre personne. [Sondez : éviter la discrimination, partager les responsabilités généralement séparées selon le genre, décider de ne jamais contraindre personne à avoir des rapports sexuels ou écrire une lettre d'excuses à quelqu'un qu'on n'aurait pas bien traité dans le passé.]

- Avez-vous été témoins d'une situation où quelqu'un a fait une différence utile, grande ou petite?

7 Rappelez aux élèves que l'activisme est source de récompenses personnelles mais aussi de risques :

- Quelles sont les récompenses du militantisme ou de l'action pour la justice sociale? [Sondez: sentiment de pouvoir faire quelque chose pour autrui, établissement de nouvelles relations, renforcement d'aptitudes personnelles telles que la communication en public ou par écrit, admiration des autres.]

- Quels peuvent être les risques du militantisme ou de l'action pour la justice sociale? [Sondez: risque de désapprobation sociale, distraction $d u$ travail scolaire ou d'autres aspects importants de la vie, déception si on n'arrive pas au but visé, risque d'arrestation ou autres dangers, même si l'activité est parfaitement légale et pacifique. Rappelez aux élèves qu'il est important de rester en sécurité.]

8 (Peut être donné comme devoir.) Distribuez la feuille «Lettre du fond du cœur » ou écrivez les points à développer au tableau. Dites aux élèves :

- Écrivez une lettre, du fond du cœur, sur une cause qui vous est chère et ce que vous pouvez y faire.

- Adressez cette lettre à vous-même, à un parent, un ami, un partenaire ou un leader communautaire. 


\section{une cause qui me tient à cœur}

La liste ci-dessous présente une série de problèmes sociétaux dont la résolution est chère à beaucoup. Notre liste se concentre sur trois catégories : le genre, la santé sexuelle et les droits sexuels. Réfléchissez aux causes qui vous tiennent particulièrement à cœur. Peut-être pensez-vous à un problème similaire à l'un de ceux-ci, ou à une toute autre cause?

\section{Cochez trois causes qui vous tiennent à cœur (toutes dans la même catégorie ou dans des catégories différentes).}

\section{GENRE}

$\square$ Nos manuels scolaires renforcent les stéréotypes.

$\square$ Certaines politiques de notre école sont discriminatoires à l'égard de certains groupes.

$\square$ L'école tolère trop de taquineries et de brimades.

$\square$ Dans notre communauté, les garçons se sentent sous pression d'être « durs » ou intrépides.

$\square$ Les garçons sont sous pression de se joindre à un gang ou de prouver leur virilité.

$\square$ Les filles n'ont pas l'occasion d'apprendre ce qu'elles devraient savoir sur la gestion de l'argent.

$\square$ Les filles n'ont pas suffisamment l'occasion de faire du sport ou d'être sélectionnées dans une équipe.

$\square$ Les filles ne disposent pas d'espaces sûrs où rencontrer leurs amies et leurs pairs.

$\square$ La publicité représente le sexe masculin et le sexe féminin de manière nuisible à notre bien-être.

$\square$ Trop peu de jeunes hommes ont l'occasion d'apprendre comment être de bons pères.

$\square$ La violence sexiste (contre les femmes et les filles) est trop répandue et elle est même acceptée.

$\square$ Les hommes violents envers leur femme et leurs enfants sont souvent plus violents encore sous l'influence de l'alcool.

$\square$ Les normes de genre contribuent aux troubles du comportement alimentaire.

$\square$ Trop de femmes et de filles recourent à la chirurgie esthétique pour se conformer à une image idéale.

$\square$ Les filles n'ont pas les mêmes chances que leurs frères d'aller à l'école.

$\square$ Les filles enceintes ne sont pas autorisées à poursuivre leurs études.

$\square$ Les garçons et les filles ne sont pas traités sur un pied d'égalité à la maison.

$\square$ Les membres de la communauté ne comprennent pas suffisamment le problème de l'inégalité de genre.

$\square$ Trop peu de gens sont conscients du problème du harcèlement sexuel.

$\square$ AUTRE : 


\section{une cause qui me tient à cœur}

\section{SANTÉ SEXUELLE}

$\square$ Les adolescents n'ont pas accès à des services de santé sexuelle et génésique sensibles à leurs besoins.

$\square$ Il est difficile de se procurer des préservatifs.

$\square$ Beaucoup de mes pairs ne savent rien du VIH.

$\square$ Dans notre région, beaucoup d'écoles ne parlent pas du VIH.

$\square$ Beaucoup de gens ignorent leur état $\mathrm{VIH}$.

$\square$ Les jeunes ne disposent même pas d'une information élémentaire sur leur propre corps.

$\square$ Les taux d'infections sexuellement transmissibles relevés chez les jeunes sont bien trop élevés.

$\square$ Dans certaines régions du monde, beaucoup de femmes et de filles courent le risque de la fistule obstétricale.

$\square$ Les gens ignorent le problème de la mortalité maternelle ou ne s'en soucient pas suffisamment.

$\square$ L'avortement est limité par la loi - et est par conséquent dangereux — dans de nombreux endroits.

$\square$ AUTRE :

\section{DROITS SEXUELS}

$\square$ Le problème de l'inceste est largement ignoré dans de nombreuses sociétés.

$\square$ Trop de filles sont mariées alors qu'elles sont encore enfants.

$\square$ La mutilation génitale des filles se pratique toujours.

$\square$ Beaucoup de filles subissent l'exploitation sexuelle de « sugar daddies ».

$\square$ Beaucoup de jeunes, en particulier les filles, sont victimes du trafic sexuel.

$\square$ Le viol est trop fréquent et même toléré.

$\square$ Les gens ne se rendent pas compte que les garçons courent aussi le risque d'abus sexuels.

$\square$ Les homosexuels ne sont pas souvent traités avec dignité.

$\square$ Beaucoup de jeunes, en particulier les filles, ne pensent pas qu'elles ont vraiment le droit d'insister sur l'usage du préservatif.

$\square$ Les personnes vivant avec le VIH et le sida ne bénéficient pas d'un soutien et d'un respect suffisants.

$\square$ AUTRE :

N'oubliez pas d'écrire votre nom sur les deux pages de cet exercice!

176 


\section{lettre du fond du cœur}

REMARQUE : Le changement positif peut prendre de nombreuses formes. N'oubliez pas qu'il est important de « commencer petit ». II est important aussi de choisir une action sûre et légale. En voici quelques exemples:

\section{Au niveau personnel}

Décider de ne pas faire de discrimination

Accompagner un(e) ami(e) dans un centre de soins

Promettre de ne pas être violent envers autrui

Écouter quelqu'un qui a besoin de parler

En apprendre plus sur la cause qui m'est chère

Parler de ma cause à mes amis et à ma famille

\section{Pour un projet de plaidoyer}

Former un petit groupe d'action sociale

Écrire une lettre à un représentant public

Écrire un article pour mes pairs

Rejoindre une organisation vouée à la cause qui m'est chère

Prier mon école de changer une politique particulière

Planifier une manifestation dans la communauté

Cher / Chère

J'apprends ce que représentent l'égalité de genre, la santé et les droits sexuels. Je me rends compte qu'il en faut peu pour opérer un changement positif et utile. Je m'intéresse particulièrement à la cause de

Pour opérer un changement positif, je peux [choisissez une action dans la liste ci-dessus ou formulez une idée différente]

Mon espoir est que

Je désire être une personne qui

En toute sincérité, 


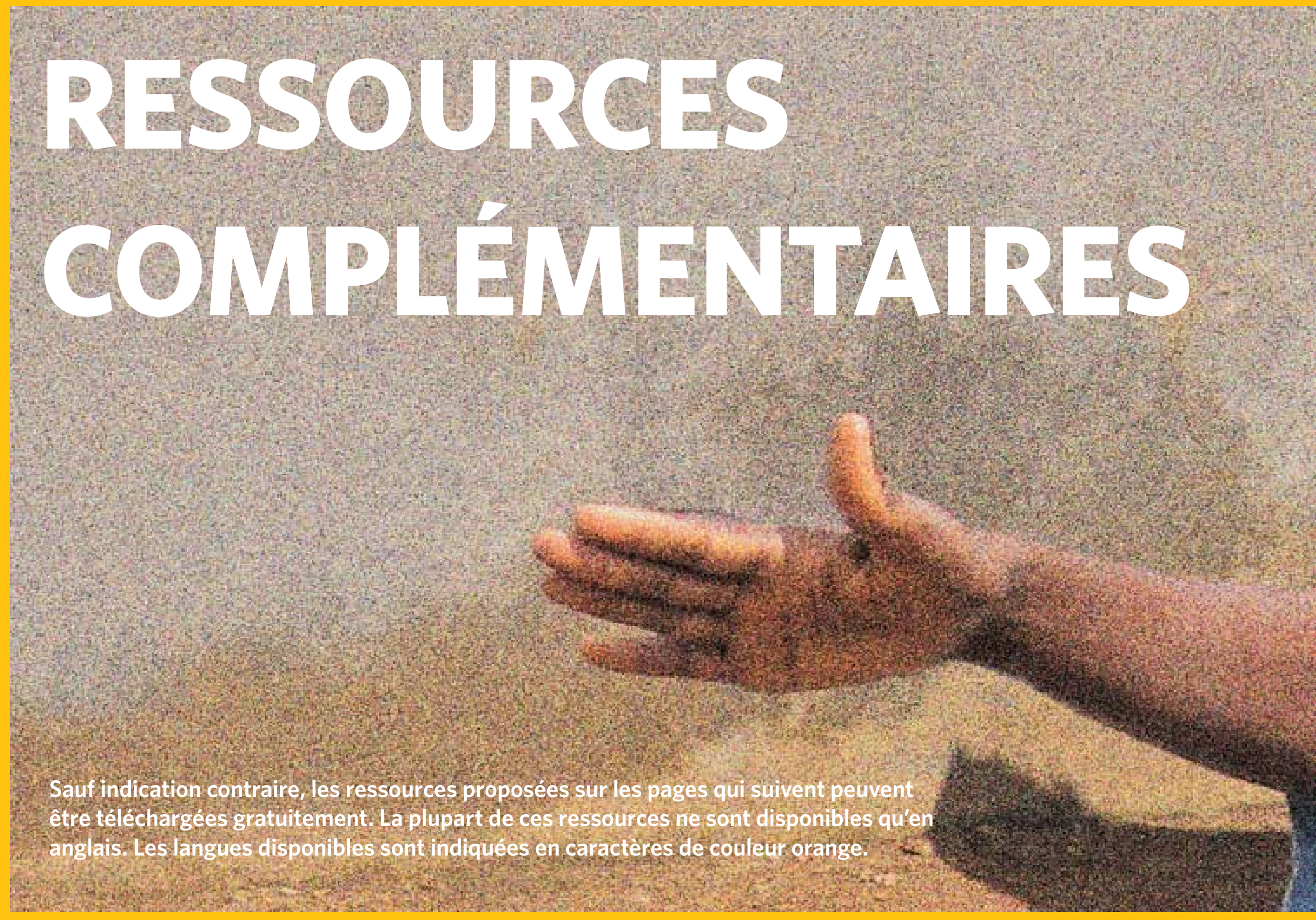




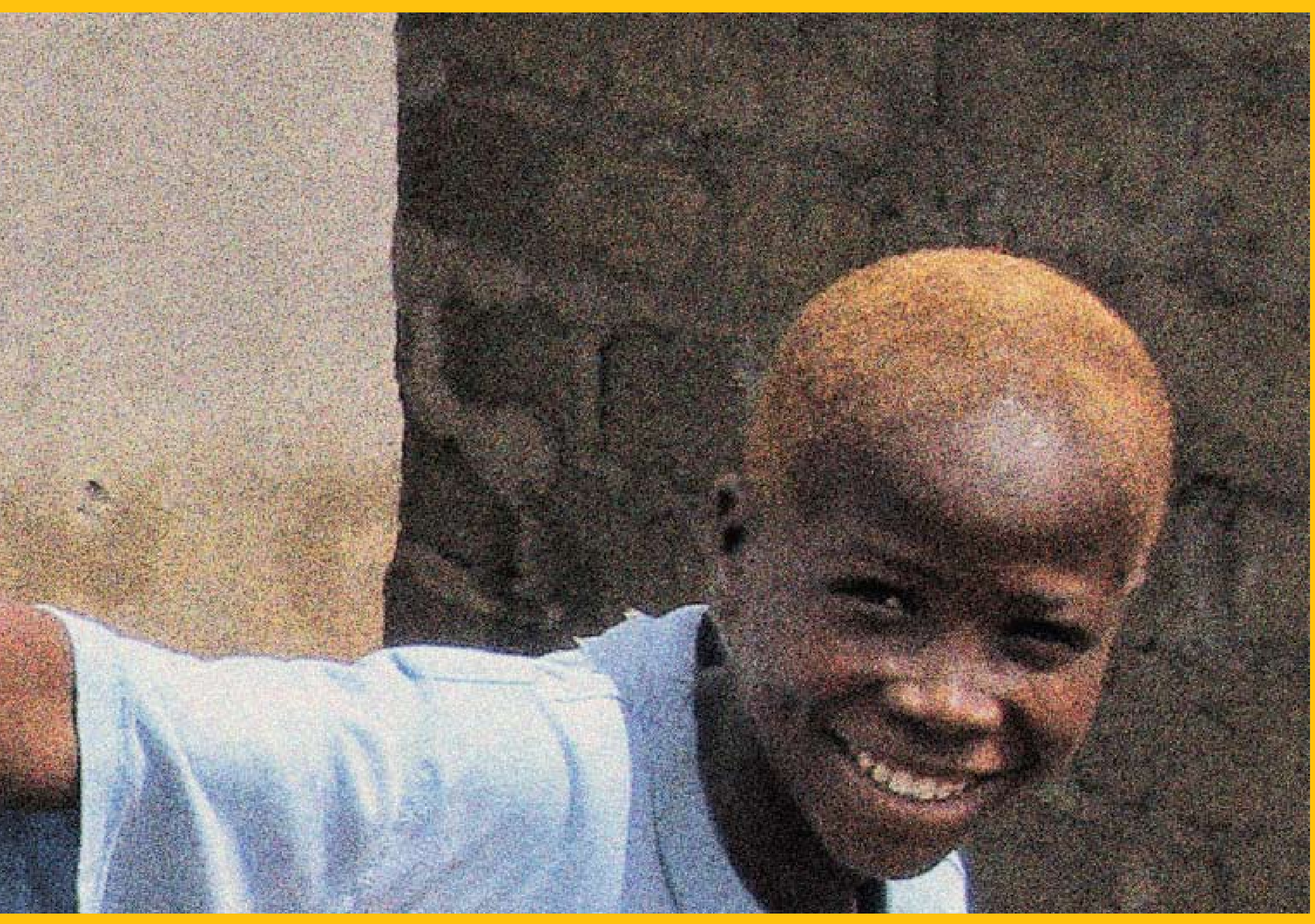


Aahung au Pakistan, entreprend des projets de formation, recherche et plaidoyer pour créer un environnement où les droits sexuels de chaque individu sont respectés, protégés et pleinement satisfaits. <www.aahung.org $>$. En anglais.

Conscientizing Male Adolescents au Nigeria, cherche à sensibiliser les adolescents de sexe masculin aux préjugés et pratiques sexistes et à développer en eux les attitudes et les compétences nécessaires au changement. Voir à ce sujet Q/C/Q n 14 : <www.popcouncil.org/pdfs/qcq/qcq14fr.pdf>. En français et en anglais.

Dance4Life engage danseurs, musiciens, pairs éducateurs et jeunes vivant avec le $\mathrm{VIH}$ à informer et autonomiser les jeunes dans la lutte contre le VIH et le sida. $<$ www.dance4life.com>. En anglais.

Democracia y Sexualidad (DEMYSEX) est un réseau d'organisations mexicaines voué au renforcement de l'éducation sexuelle pour assurer l'équité sexuelle et l'exercice des droits sexuels au sein d'une culture démocratique. <www.demysex. org.mx>. En anglais et en espagnol.

Girls Power Initiative au Nigeria, vise à donner aux filles l'information, les capacités et les occasions d'action qui leur permettent de s'épanouir en toute confiance et compétence. <www.gpinigeria.org>. En anglais.

Girls Incorporated aux États-Unis, organise des programmes éducatifs destinés aux filles, dans les milieux défavorisés à hauts risques surtout, pour les aider à surmonter la discrimination sexuelle et à mener une vie indépendante et épanouie. $<$ www.girlsinc.org $>$. En anglais.

\section{International Centre for Reproductive Health and Sexual Rights (INCRESE) au}

Nigeria, cherche à étendre l'accès à l'information sur la santé et les droits sexuels et aux services. <www.increse-increse.org>. En anglais.

MenEngage est une alliance mondiale vouée à l'engagement des hommes et des garçons dans la lutte contre les inégalités de genre et pour la santé et le bien-être des femmes, des hommes et des enfants. <www.menengage.org>. En anglais.

Raising Voices, en Ouganda, œuvre pour la prévention de la violence à l'encontre des femmes en s'attaquant à ses causes profondes, y compris les rôles de genre traditionnels et le déséquilibre du pouvoir entre les femmes et les hommes. <www. raisingvoices.org>. En anglais.

Scenarios USA (inspiré de Scenarios du Sahel) parraine un programme et un concours de scénario inspiré de questions de genre. Les scénarios gagnants servent à la réalisation de courts métrages. Le programme « What's the Real Deal about Masculinity? » et les films sont disponibles à l'achat. Les films peuvent aussi être visionnés gratuitement en ligne. <www.scenariosusa.org $>$. En anglais.

Sonke Gender Justice Network œuvre avec les hommes, les femmes, les jeunes et les enfants d'Afrique australe en faveur de l'égalité de genre, contre la violence sexiste et contre le VIH et le sida. <www.genderjustice.org.za>. En anglais.

White Ribbon Campaign cherche à éduquer les hommes et les garçons pour mettre fin à la violence contre les femmes. <http://whiteribbon.ca/Francais>. En français et en anglais. 
ABC: L'enseignement des droits de l'homme-Activités pratiques pour les écoles primaires et secondaires, Haut-Commissariat des Nations Unies aux droits de I'homme. 2003. 124 pages. Ce manuel aide les enseignants à éveiller la conscience et à encourager l'action pour les droits humains. <www.unhchr.ch/hredu.nsf/GV04f r/9CF6357A173AEAF8C1256D04004BC0BB>. En français, en anglais, en arabe, en chinois, en espagnol et en russe.

African Transformation: The Way Forward, Facilitator's Workshop Guide, Health Communication Partnership, USAID et Communication for Development Foundation Uganda, 2005. 118 pages. Outils pour la conduite d'ateliers sur les normes de genre dans nos vies. <www.popline.org/ics-wpd/mmc/media/PLAFR302.pdf>. En anglais.

Construyendo Derechos: Talleres de Conversación Para Adolescentes, FLACSO et UNFPA, 2006. 90 pages. Instructions pour discussions guidées sur huit aspects de la sexualité et des droits. <www.issuu.com/flacso.chile/docs/construyendo_ derechos/15>. En espagnol seulement.

Donner aux jeunes femmes le pouvoir d'initier le changement, World YWCA et UNFPA, 2006. 124 pages. Pour les animateurs désireux d'aider les jeunes femmes à se poser en leaders et pour catalyser le changement positif dans leur vie et dans leurs communautés. <www.worldywca.org/world_ywca/communications/ resources/empowering_young_women_to_lead_change>. En français, en anglais et en espagnol.

Faire participer les garçons et les hommes à la transformation des rôles masculins et féminins: manuel d'éducation pour le travail en équipe, EngenderHealth et Promundo, 2008. 356 pages. Exercices de participation des hommes (et de leurs partenaires), exploration de la socialisation liée au genre et de son impact sur la prévention et les soins du VIH. <www.acquireproject.org/archive/files/7.0_engage_ men_as_partners/7.2_resources/7.2.3_tools/group\%20education\%20manual_ francais_web_final.pdf>. En français et en anglais.

Filling the Gaps: Hard to Teach Topics in Sexuality Education, Sexuality Information and Education Council of the United States, 1998. 193 pages. Ce manuel propose des plans de leçon sur l'abstinence, les options de grossesse, le comportement sexuel et I'identité sexuelle. <www.siecus.org/pubs/filling_the_gaps.pdf>. En anglais.
Gendering Prevention Practices: A Practical Guide to Working with Gender in Sexual Safety and HIV/AIDS Awareness Education, Nordic Institute for Women's Studies and Gender Research, 2003. 52 pages. Ce manuel vise à sensibiliser les jeunes aux questions de genre au profit de comportements sexuels plus sûrs.<www.nikk.no/ filestore/Publikasjoner/Living_for_Tomorrow/Ift_gendprevpract.pdf $>$. En anglais.

Gender or Sex: Who Cares?, Ipas et Health and Development Networks, 2001. 96 pages. Ressource de formation destinée à accroître les compétences des délégués à la jeunesse sur le genre et la santé génésique. <www.ipas.org/Publications/ Gender_or_sex_Who_cares.aspx?ht>. En anglais et en espagnol.

Human Rights Education Series, Human Rights Resource Center, University of Minnesota, 2000. Le troisième volume de cette série de six, publiée en collaboration avec Amnesty International USA et le réseau Gay, Lesbian and Straight Education Network, traite de la diversité et des droits sexuels. <www1. umn.edu/humanrts/edumat>. En anglais.

International Programme on Sexuality Publications, Youth Incentives. Ce site propose des brochures, des fiches factuelles, des plans de leçons et des dossiers de plaidoyer. <www.youthincentives.org/rutgersnisso_groep/youthincentives/ Downloads>. En français et en anglais.

Keep the Best Change the Rest: Participatory Tools for Working with Communities on Gender and Sexuality, Recueil d'activités destinées à aider les communautés à explorer la manière dont le genre et la sexualité affectent la vie de chacun et à identifier les moyens d'améliorer les relations et de protéger la santé sexuelle. <http://pdf.usaid.gov/pdf_docs/PNADJ846.pdf>. En anglais.

MediaLitKit $^{\mathrm{TM}}$ Framework for K-12 Media Literacy, Center for Media Literacy, 2005. 137 pages. Ce kit présente une explication de la pédagogie médiatique et propose des stratégies de mise en œuvre. <www.medialit.org $>$. En anglais. Plans de leçon proposés sur <www.medialit.org/reading_room/rr4_lessonplan.php>. En anglais et en espagnol.

Nouvelle édition de <<Mon corps qui change>>, Institute for Reproductive Health, à paraître. Ce programme informe les jeunes sur la puberté et les changements qui affectent leur corps. L'accent est mis, en particulier, sur la menstruation et la conscience de la fécondité. <www.irh.org>. En français, en anglais et en espagnol. 
One Man Can Workshop Activities: Talking to Men about Gender, Domestic and Sexual Violence and HIV/AIDS, Sonke Gender Justice, 2006. 48 pages. Ce manuel propose des activités destinées à encourager les hommes et les garçons à réfléchir à leurs propres attitudes et comportements à l'égard du genre, des femmes, de la violence familiale et sexuelle, du VIH/sida, de la démocratie et des droits humains. <www.genderjustice.org.za/onemancan/complete-oneman-can-toolkit/download-the-complete-to.html>. En français, en afrikaans, en anglais, en isiXhosa et en isizulu.

\section{Our Future: Sexuality and Life-skills Education for Young People, Grades} 4-5, Grades 6-7, and Grades 8-9, International AIDS Alliance. 2006 et 2007. 132 pages, 128 pages, et 150 pages. Trois volumes d'information et d'activités pédagogiques sur la puberté, l'amitié, le genre, la sexualité, la grossesse, les infections sexuellement transmissibles, le VIH, le sida et la drogue. Idées, aussi, pour la préparation des parents et tuteurs à la discussion de la sexualité avec leurs enfants. <www.aidsalliance.org/publicationsdetails.aspx?id=211>. En anglais.

\section{People's Movement for Human Rights Education (PDHRE-International)}

$œ u v r e$ pour le développement et la promotion de l'éducation aux droits humains. L'organisation publie des manuels de formation et un matériel pédagogique. $<$ www.pdhre.org>. En anglais.

Rights and Desire: A Facilitator's Manual to Healthy Sexuality, Breakthrough, 2006. 106 pages. Ce manuel vise à produire un dialogue constructif sur les relations, le sexe et la sexualité. <www.breakthrough.tv/images/downloads/53/ rights_and_desire.pdf $>$. En anglais.

Sakhi Saheli - Promoting Gender Equity and Empowering Young Women: A Training Manual, CORO for Literacy, Horizons/Population Council et Instituto Promundo. 2008. 136 pages. Manuel de réflexion pour aider les jeunes femmes à comprendre comment les normes de genre affectent leur vie et accroissent leur vulnérabilité au VIH et à d'autres problèmes de santé génésique. <www. popcouncil.org/pdfs/horizons/India_SakhiSaheli_Eng.pdf>. En anglais.

\section{Sexuality and Life-skills: Participatory Activities on Sexual and Reproductive} Health with Young People, International AIDS Alliance. 2008. 172 pages. Activités destinées à aider les jeunes à développer les connaissances, les attitudes positives et les compétences utiles à l'épanouissement de leur santé et bien-être sexuels et génésiques. <www.aidsalliance.org/publicationsdetails. aspx?id=295>. En anglais.

\section{Stepping Stones: A Training Package on HIV/AIDS, Communication and}

Relationship Skills, ACTIONAID, 1995. 240 pages. £11.25. Manuel de formation pour l'organisation d'un atelier d'exploration des besoins sociaux, sexuels et psychologiques et la pratique de différents comportements dans les relations. $<$ www.steppingstonesfeedback.org>. En vente sur <www.talcuk.org/books/bsstepping-stones.htm>. En anglais.

Tools for Change: An Educator's Resource Site, Centre for Research and Education on Violence Against Women and Children à I'University of Western Ontario. Ce site propose une liste de ressources pédagogiques (8 à 15 ans) pour le développement de relations saines et égales. <www.toolsforchange.ca>. En anglais.

Working with Young Men Series, Project H of Instituto Promundo, with ECOS Comunicação em Sexualidade, Programa de Apoio ao Pai (PAPAI), Salud Y Género. 314 pages. Guides d'éducation des jeunes hommes de 15 à 24 ans sur les rôles de genre, la violence et la sexualité. <www.promundo.org.br/396>. En portugais, en espagnol et en anglais.

Working with Young Women: Empowerment, Health, and Rights, Instituto Promundo, Salud y Género, ECOS, Instituto PAPAI et World Education. 2009. 143 pages. Manuels d'activités sur l'égalité de genre et les droits des femmes, à I'intention des jeunes femmes de 15 à 24 ans. <www.promundo.org.br/352>. En portugais et en anglais.

Yaari Dosti - Young Men Redefine Masculinity, Population Council, CORO for Literacy, MAMTA et Instituto Promundo, 2006. 110 pages. Manuel de promotion de l'égalité de genre en tant que stratégie pour la prévention du $\mathrm{VIH}$. $<w w w$.popcouncil.org/pdfs/horizons/yaaridostieng.pdf>. En anglais. Également disponible en hindi sur <www.popcouncil.org/pdfs/horizons/yaaridostihindi.pdf>.

Young Men and HIV Prevention: A Toolkit for Action, Promundo et UNFPA. 2007. 115 pages et 38 pages. Information conceptuelle et pratique sur la conception, la mise en œuvre et l'évaluation d'activités de prévention du VIH et du sida qui incorporent une perspective de genre et engagent les jeunes hommes. <www. promundo.org.br/352>. En portugais, en espagnol et en anglais. 


\section{formation et perfectionnement des enseignants}

Doorways: School-Related Gender-Based Violence Prevention and Response, USAID 2009. Série de trois manuels (pour enseignants, élèves et conseillers communautaires) pour la sécurité à l'école à travers un partenariat communauté- école. <www.usaid. gov/our_work/cross-cutting_programs/wid/doorways.html>. En anglais.

Formation des formateurs pour le développement, Centre for Development et Population Activities, 1995. 93 pages. Ce manuel couvre un atelier de six jours sur les techniques de formation participative. <www.cedpa.org/uploaded_files/ trainingtrainers_french_all.pdf>. En anglais et en espagnol.

Gender, HIV, and Human Rights: A Training Manual, UNIFEM, UNFPA, UNAIDS, 2000 213 pages. Ce manuel couvre une formation de un et deux jours, pour aider les éducateurs à mieux comprendre les dimensions de genre et de droits humains de la pandémie du VIH. <www.unifem.org/resources/item_detail.php?ProductID=5>. En anglais.

Gender or Sex: Who Cares?: Notes for Training of Trainers, Ipas, 2002. 68 pages. Ces notes de ressources aident les animateurs expérimentés à mener la formation Gender or Sex: Who Cares? (voir programmes pédagogiques plus haut). <www.ipas org/Publications/Gender_or_sex_Notes_for_Training_of_Trainers.aspx?ht>. En anglais, en créole et en espagnol.

The Human Rights Education Handbook: Effective Practices for Learning, Action, and Change, the Human Rights Resource Center, University of Minnesota, 2000. Ce manuel guide les éducateurs à enseigner efficacement la matière relative aux droits humains. <www1.umn.edu/humanrts/edumat/hreduseries/hrhandbook/toc.html>. En anglais.

Our Future: Preparing to Teach Sexuality and Life Skills, An Awareness Training Manual for Teachers and Community Workers, International AIDS Alliance, 2008. 94 pages. Prépare les éducateurs à l'enseignement du programme Our Future (voir les programmes pédagogiques et activités plus haut). <www.aidsalliance.org/ publicationsdetails. aspx?id=293>. En anglais

Training of Trainers: Designing and Delivering Effective Human Rights Education, Training Manual, Equitas - International Centre for Human Rights Education, 2007. 202 pages. Grandes lignes d'un atelier expérientiel de six jours sur la formation des formateurs à la sensibilisation aux droits humains. <www.equitas.org/english/ ed-manuals/ed-manuals.php>. En anglais. Également disponible en russe (édition française à paraître)

\section{lectures sur I'enseignement et l'apprentissage}

Experiential Learning Cycles: Overview of 9 Experiential Learning Cycle Models. Ce site présente neuf modèles d'apprentissage expérientiel et leur application. <www.wilderdom.com/experiential/elc/

ExperientialLearningCycle.htm>

Gold Dust Resources, Quality Improvement Agency. Ce site propose des ressources, fiches documentaires et activités vidéo, de perfectionnement des éducateurs de tous niveaux d'expérience. <www.goldust.org.uk>. En anglais.

Hesperian Foundation. Publie des ouvrages et lettres d'information sur les causes sociales profondes de la santé précaire et suggère des modes d'organisation de groupes communautaires pour l'amélioration des conditions sanitaires. $<$ www.hesperian.org/publications_download.php>.

Documents proposés dans 152 langues.

The Learner-centered Teaching Series, Teaching Effectiveness Program, University of Oregon. Ce site présente une série en quatre parties sur l'enseignement axé sur l'apprenant (aperçu général, développement de programme, contenu et évaluation des élèves). <http://tep.uoregon.edu/ workshops/teachertraining/learnercentered/learnercentered.html>. En anglais.

Paulo Freire and Informal Education, the Encyclopaedia of Informal Education, 2002. Cette page Web présente une introduction aux travaux de Paolo Freire, avec références et liens complémentaires. <www.infed.org/thinkers/et-freir.htm>. En anglais.

Training for Transformation, Volumes 1-3, ITDG Publishing, 2002. 462 pages. $\$ 50$. Cet ouvrage en trois volumes de techniques de formation innovantes intègre différentes approches et méthodologies d'enseignement participatif, développement organisationnel et autonomie communautaire. En vente sur le site de Hesperian Foundation <www.hesperian.org/mm5/merchant.mvc? Screen=PROD\&Store_Code $=$ HB\&Product_Code $=$ B803\&Category_Code $=H E B>$ En anglais. 
Le cadre de I'IPPF pour une éducation sexuelle intégrée, International Planned Parenthood Federation, 2006. 9 pages. Introduction à l'éducation complète à la sexualité et cadre fondamental de mise en œuvre. <www.ippf.org/ en/Resources/Guides-toolkits/Framework+for+Comprehensive+Sexuality+ Education.htm>. En français et en espagnol.

CARE, CARE publie des ouvrages pertinents dont, notamment, S'attaquer aux facteurs sociaux influençant la santé sexuelle et reproductive et ISOFI : Outils pour l'apprentissage et l'action sur le genre et la sexualité (pour aider les personnels à explorer les questions du genre et de la sexualité). <www.care.org/careswork/ whatwedo/health/srh/publications.asp $>$. En français et en espagnol.

Developing Guidelines for Comprehensive Sexuality Education, Sexuality Education and Information Council of the United States, 1999. 36 pages. Ce guide apporte aux éducateurs, décideurs et activistes une procédure détaillée d'élaboration de directives concernant les programmes d'éducation à la sexualité. $<$ www.siecus.org/data/global/images/guideintl.pdf>. En anglais,

\section{Dynamic Contextual Analysis of Young People's Sexual Health:} A Context Specific Approach to Understanding Barriers to, and Opportunities for, Change, Thomas Coram Research Unit, University of Southampton, 2006. 51 pages. Décrit comment effectuer une analyse contextuelle dynamique des facteurs qui affectent la vie sexuelle des jeunes, pour servir de base à la programmation. <www.safepassages.soton.ac.uk/pdfs/DCA2.pdf>. En anglais.

Education sur le VIH/SIDA: Perspective sexospécifique, UNICEF, 2002.

24 pages. Introduction, listes et activités destinées à la formation des éducateurs dans les contextes formels et informels. <www.unicef.org/lifeskills/files/UNICEF_ GenderHIV_Fren.pdf>. En français, en anglais, et en espagnol.

Facing the Challenges of HIV, AIDS, STDs: A Gender-based Response, Royal Tropical Institute, Southern Africa AIDS Information Dissemination Service et World Health Organization, Amsterdam, 1995. 56 pages. Information et liste à l'intention des décideurs et des exécutants des programmes concernant I'incorporation du genre dans les politiques et programmes relatifs au $\mathrm{VIH}$, au sida et autres IST. <http://data.unaids.org/Topics/Gender/FacingChallenges_en.pdf>. En anglais et en hindi.
Key Issues in the Implementation of Programmes for Adolescent Sexual and Reproductive Health, Department of Child and Adolescent Health and Development, World Health Organization, 2004. 51 pages. Examen de questions programmatiques relatives à la santé sexuelle et génésique des adolescents.

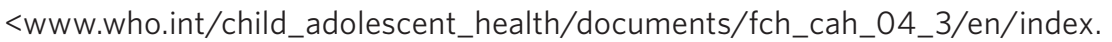
html>. En anglais.

Lignes directrices canadiennes pour l'éducation en matière de santé sexuelle, Agence de la santé publique du Canada, 2003. Exemple de la manière dont un gouvernement aborde l'éducation à la sexualité. <www.phac-aspc.gc.ca/publicat/ cgshe-Idnemss/index-fra.php>. En français et en anglais.

RHIYA (The Reproductive Health Initiative for Youth in Asia), initiative passée de I'Union européenne et UNFPA en Asie du Sud et du Sud-Est, pour l'amélioration de la santé sexuelle et génésique des jeunes de 10 à 24 ans. Le projet a produit une série d'études de cas et de rapports de mise en œuvre programmatique $<$ www.unfpa.org/eu_partnership/rhiya $>$. En anglais.

\section{Synergizing HIV/AIDS and Sexual and Reproductive Health and Rights -} A Manual for NGOs, AIDSNET, 2006. 22 pages. Données et directives pour I'intégration de la santé sexuelle/génésique, des droits et du genre dans la lutte contre le VIH. <www.aidsnet.dk/Default.aspx?ID=2366>. En anglais.

Toolkit for Mainstreaming HIV and AIDS in the Education Sector: Guidelines for Development Cooperation Agencies, Joint UN Programme on HIV/AIDS, 2008. 75 pages. Ressources et matériel de formation. <http://unesdoc.unesco.org/ images/0015/001566/156673E.pdf>. En anglais. 


\section{formation et assistance technique}

Africa Regional Sexuality Resource Centre (ARSRC), (Lagos, Nigeria) organise des instituts consacrés à la sexualité en Afrique. <www.arsrc.org/training/asi/ background.htm>. En anglais.

\section{Association suédoise pour l'éducation sexuelle (RFSU) apporte assistance} technique et formation sur la santé et les droits sexuels et génésiques des jeunes, la diversité sexuelle et les droits humains. <www.rfsu.se/fr/Francais>. En français, en suédois, en anglais et en espagnol.

Creating Resources for Empowerment in Action (CREA) cherche à autonomiser les femmes, pour qu'elles articulent, exigent et obtiennent leurs droits humains à travers une assistance et formation aux droits humains des femmes, au genre et à la sexualité. Établie en Inde, CREA organise des formations annuelles de par le monde <http://web.creaworld.org>. En anglais.

Girls Power Initiative, (Calabar, Nigeria) organise un « Gender Development Institute » de sensibilisation au genre, aux droits et à la sexualité. <www.gpinigeria. org>. En anglais et en espagnol.

Global Youth Coalition on HIV/AIDS est un réseau de 4000 jeunes leaders et alliés adultes présent dans 150 pays du monde. GYCA forme et autonomise les jeunes leaders pour qu'ils élargissent leurs interventions contre le VIH et le sida parmi leurs pairs. <www.youthaidscoalition.org>. En anglais et en espagnol.
Instituto Promundo, (Brésil) est à la base de Projeto $\mathrm{H}$ et Projeto $\mathrm{M}$ sur le genre, les droits et la santé sexuelle des jeunes hommes et femmes. Ces projets apportent formation et assistance technique à l'éducation sur le genre et la sexualité <www. promundo.org.br>. En anglais, en espagnol et en portugais.

\section{International School for Humanities and Social Sciences, Universiteit van}

Amsterdam, mène des recherches et organise un Summer Institute on Sexuality, Culture, and Society pour l'exploration des dimensions sociales de la sexualité à travers les cultures. Des bourses sont disponibles aux participants de certains pays $<w w w . i s h s s . u v a . n l / S u m m e r \mid n s t i t u t e / i n d e x . h t m l>$. En anglais.

National Sexuality Resource Center, San Francisco State University propose un Summer Institute on Sexuality and Culture. <http://nsrc.sfsu.edu>. En anglais.

The Pleasure Project, établi à Oxford (Royaume-Uni) et en Inde, propose formation et assistance technique aux formateurs et conseillers sur l'adoption d'une approche sexuelle positive de leur travail. <www.thepleasureproject.org>. En anglais.

Reprolatina, (Brésil) propose ses formations au genre, à la santé et aux droits sexuels et génésiques en Bolivie, au Brésil, au Chili et au Paraguay.

$<$ www.reprolatina.org.br>. En portugais.

Talking about Reproductive and Sexual Health Issues (TARSHI), (Inde) apporte une formation sur la sexualité, le genre et les droits dans la région d'Asie du Sud et du Sud-Est. <www.tarshi.net>. En anglais et en hindi. 
Action Canada pour la population et le développement (ACPD) œuvre pour le développement centré sur la santé et les droits sexuels et génésiques. <www.acpd.ca> En français et en anglais

Advocates for Youth milite en faveur de politiques qui permettent aux jeunes de prendre des décisions responsables, en connaissance de cause, concernant leur santé sexuelle et génésique, aux États-Unis et dans le reste du monde. <www. advocatesforyouth.org>. En anglais.

Amnesty International, organisation pour les droits humains, milite pour l'accès des filles à l'éducation sans risques à travers sa campagne pour des Écoles plus sûres. Pour un rapport et des fiches d'information, voir <www.amnesty.org/fr/campaigns/stopviolence-against-women/issues/empowerment-women/safe-schools $>$. En français.

BRIDGE, Institute for Development Studies, comble l'écart entre la théorie, la politique et la pratique par l'apport d'une information accessible et diverse sur le genre, sous forme imprimée et en ligne. <www.bridge.ids.ac.uk>. En anglais

Catholics for Choice (CFC) milite pour une éthique sexuelle et génésique basée sur la justice, un engagement pour le bien-être des femmes et la capacité morale des personnes à prendre de bonnes décisions pour elles-mêmes et leur vie. <www. catholicsforchoice.org>. En anglais.

Center for Health and Gender Equity (CHANGE) milite en faveur de politiques et programmes internationaux du gouvernement des États-Unis propices à la santé et aux droits sexuels et génésiques. <www.genderhealth.org/index.php>. En anglais.

Center for Reproductive Rights est une organisation de plaidoyer juridique qui milite à l'échelle mondiale. Pour ses publications sur les droits sexuels et génésiques des adolescents, voir : <www.reproductiverights.org/pdf/adolescents\%20bp_FINAL.pdf>, $<$ www.reproductiverights.org/pdf/SexualityEducationforAdolescents.pdf $>$ et $<w w w$. reproductiverights.org/pdf/BRB_SexEd.pdf>. En anglais.

CHOICE for Youth and Sexuality est une organisation de jeunes néerlandaise qui milite pour la santé et les droits sexuels et génésiques des jeunes. $<$ www.choiceforyouth.org $>$. En anglais et en néerlandais

Family Care International œuvre pour la prévention de la mortalité et des séquelles de la grossesse et de l'accouchement et pour la santé et les droits sexuels et génésiques partout dans le monde. <www.familycareintl.org/fr/home>. En français, en anglais et en espagnol.

Family Violence Prevention Fund œuvre pour mettre fin à la violence faite aux femmes et aux enfants dans les foyers et les communautés, et pour aider ses victimes, aux États-Unis et dans le monde. <www.endabuse.org>. En anglais.
Human Rights Watch œuvre pour la protection des droits humains partout dans le monde, y compris les droits des femmes, des homo-, bi- et transsexuels, et pour les droits relatifs à la santé, y compris le VIH. <www.hrw.org/fr >. En français.

International Gay and Lesbian Human Rights Commission (IGLHRC) milite pour la reconnaissance complète des droits humains de tous et de toutes, indépendamment de leur orientation ou expression sexuelle, identité ou expression de genre, ou état $\mathrm{VIH}$. <www.iglhrc.org $>$. En anglais

International Lesbian and Gay Association (ILGA) est un réseau mondial d'organisations vouées à l'accès aux droits égaux pour les homo-, bi-, trans- et intersexuels. <www.ilga.org/ilga/fr/index.html>. En français. Également disponible en anglais et en espagnol.

International Planned Parenthood Federation (IPPF), un réseau mondial d'associations membres, assure des soins de santé sexuelle et génésique et militent pour la santé et les droits sexuels et génésiques pour tous. <www.ippf.org/en>. En anglais.

International Women's Health Coalition (IWHC) œuvre pour l'adoption de politiques et programmes démographiques et de santé et l'allocation de ressources propices à la promotion et à la protection des droits et de la santé des filles partout dans le monde. $<$ www.iwhc.org $>$. En français et en anglais.

Ipas œuvre partout dans le monde pour accroître la capacité des femmes d'exercer leurs droits sexuels et génésiques et réduire la mortalité et les séquelles de l'avortement. <www.ipas.org>. En anglais.

Sexuality Information and Education Council of the United States (SIECUS) œuvre pour l'éducation à la sexualité et la santé et les droits sexuels. <www.siecus.org $>$. En anglais.

Women's Global Network for Reproductive Rights (WGNRR) rassemble des groupes et des particuliers voués à la défense des droits génésiques des femmes. $<$ www.wgnrr.org >. En anglais.

World Association for Sexual Health (WAS) œuvre pour la promotion de la santé sexuelle pour tous à travers son organe international, ses fédérations continentales régionales et ses organisations nationales. <www.worldsexology.org>. En anglais.

Youth Coalition, est une organisation internationale de jeunes qui milite pour les droits sexuels et génésiques des jeunes à l'échelle nationale, régionale et internationale et pour la participation utile des jeunes aux décisions qui les affectent. $<$ www.youthcoalition.org $>$. En anglais.

Beaucoup des organisations reprises sur cette page proposent un matériel disponible dans plusieurs langues. 


\section{organisations régionales de plaidoyer et politique}

\section{Amanitare: African Partnership for the Sexual and Reproductive Health and}

Rights of Women and Girls œuvre pour l'intégrité corporelle et les droits sexuels et génésiques des femmes d'Afrique et cherche à attirer l'attention sur les droits des femmes en matière de $\mathrm{VIH} / \mathrm{sida}$, mortalité maternelle et contraception

$<$ www.amanitare.org.za>. En anglais.

Asian-Pacific Resource and Research Centre for Women (ARROW) préconise et défend les droits et les besoins de santé des femmes, en mettant l'accent sur la sexualité et la santé génésique. <www.arrow.org.my/>. En anglais.

\section{ASTRA - Central and Eastern European Women's Network for Sexual and} Reproductive Health and Rights, forme un réseau d'ONG et de particuliers qui militent pour la santé et les droits sexuels et génésiques, en particulier en Europe centrale et de l'Est. <www.astra.org.pl>. En anglais

Centre européen de la Jeunesse de Budapest œuvre pour l'unité européenne et la dignité de ses citoyens à travers le respect des droits humains, la démocratie pluraliste et l'État de droit. Voir la campagne «All different, all equal » : <www.coe.int/ t/dg4/eycb/default_FR.asp?>. Également disponible en anglais.

Centre régional africain de ressources sur la sexualité, sous les auspices d'Action Health Incorporated au Nigeria, cherche à contribuer au développement de programmes et politiques positifs en Afrique, à travers l'éducation, le dialogue public éclairé et le plaidoyer. <www.arsrc.org/fr/index.htm>. En français.

Centro latino-americano em sexualidade e direitos humanos dissémine la connaissance sur la sexualité dans l'optique des droits humains, pour aider à combattre l'inégalité de genre et contribuer à la lutte contre la discrimination des minorités sexuelles dans la région. <www.clam.org.br $>$. En portugais,

Femmes sous lois musulmanes est un réseau de solidarité internationale qui vise à renforcer le mouvement pour l'égalité et les droits des femmes dont la vie est façonnée, conditionnée ou régie par les lois et les coutumes dites dérivées de l'islam. $<w w w . w l u m l . o r g / f r>$. <www.wluml.org/english/index.shtml $>$. En français, autres langues accessibles.
Latin American and Caribbean Women's Health Network (LACWHN) forme un réseau d'organisations et d'individus qui militent pour la santé, les droits humains et la citoyenneté des femmes. <http://reddesalud.org > En espagnol.

National Sexuality Resource Center, à la San Francisco State University, apporte contenu, information et formations aux États-Unis dans une perspective positive de justice sociale sur la sexualité. <http://nsrc.sfsu.edu>. En anglais.

\section{SIECCAN (Sex Information and Education Council of Canada) encourage}

l'éducation publique et professionnelle sur la sexualité humaine à travers

I'information, la consultation, la recherche et la publication. <www.sieccan.org/ index.html>. En anglais.

South and Southeast Asia Resource Centre on Sexuality, sous les auspices de Talking about Reproductive and Sexual Health Issues (TAR SHI) en Inde <www. tarshi.net> cherche à accroître la connaissance sur la sexualité, la santé sexuelle et le bien-être sexuel en Asie du Sud et du Sud-Est. <www.asiasrc.org/plspk/2007_1/ at_resource_centre.asp>. En anglais.

Women for Women's Human Rights (WWHR), en Turquie, œuvre sur la scène nationale et internationale en faveur des droits des femmes. Elle soutient la Coalition pour les droits sexuels et corporels dans les sociétés musulmanes. <www.wwhr.org/ index.php>. En anglais.

YouAct, European Youth Network for Sexual and Reproductive Health and Rights, réseau de jeunes d'Europe actifs dans le domaine des droits sexuels et génésiques, cherche à autonomiser les jeunes pour faire entendre leur voix. <www.youact.org>. En anglais.

La plupart des organisations reprises sur cette page proposent un matériel disponible dans les langues propres à leur région. 


\section{Advocacy Kit for Growing Up Global: The Changing Transitions to Adulthood} in Developing Countries, Advocates for Youth et Population Reference Bureau, 2005. Ce matériel contient des fiches d'information récapitulatives des conclusions d'une analyse experte de la transition des jeunes vers l'âge adulte, avec conseils de plaidoyer. $<w w w$.advocatesforyouth.org/index. php?option=com_content\&task=v iew\&id=918\&Itemid=336> En anglais. Growing Up Global peut être commandé ou lu en ligne : <www.nap.edu/catalog.php?record_id=11174>. En anglais.

\section{Deadly Inertia: A Cross-country Study of Educational Responses to HIV/}

AIDS, Global Campaign for Education, Bruxelles, Belgique, 2005. 52 pages. Cette publication analyse la réponse du secteur de l'éducation à la pandémie du VIH. $<$ www.comminit.com/en/node/218512/38 et www.unesco.org/bpi/aids-iatt/ deadly-inertia.pdf $>$. En anglais.

\section{L'éducation à la sexualité et aux relations: vers une approche intégrée aux} études sociales, Deborah Rogow et Nicole Haberland. 2005. Sex Education 5(4): 333-344. Ce document préconise l'ancrage de l'éducation à la sexualité et aux relations dans le cadre des études sociales, avec mise en valeur du genre, du contexte social et des droits humains. En français sur: <www.popcouncil.org/pdfs/ SE_5_4_fr.pdf >. Original anglais sur: <www.popcouncil.org/pdfs/SE_5_4.pdf>.

Également disponible en espagnol sur: <www.popcouncil.org/pdfs/SE_5_4_esp.pdf>.

Gender and Sexuality Cutting Edge Pack, BRIDGE, 2006 et 2007. Cette série contient trois documents qui examinent le lien entre le genre, la sexualité et les les droits sexuels : Gender and Sexuality Overview Report (51 pages); Supporting Resources Collection (70 pages); et Gender and Development In Brief "Sexuality" (6 pages). <www.bridge.ids.ac.uk/reports_gend_CEP.html\#Sexuality>.

En français, en anglais et en espagnol.

HIV testing: The mutual rights and responsibilities of partners, Ruth DixonMueller et Adrienne Germain. 2007. Lancet 370(9602): 1808-1809. Ce commentaire préconise la reconnaissance des droits et responsabilités mutuels des deux partenaires dans les relations ou les échanges sexuels. <www.ph.ucla.edu/ EPI/seaids/lancet370_1808_1809_2007.pdf>. En anglais.
Ministerial Declaration -“Educating To Prevent." 2008. Cette déclaration reflète l'engagement commun des ministres de la Santé et de l'Éducation d'Amérique latine et des Caraïbes concernant le renforcement des efforts de prévention du VIH moyennant l'assurance de l'accès à une éducation complète à la sexualité et aux services de santé sexuelle et génésique. Elle encourage l'égalité de tous et la lutte contre la discrimination. <http://data.unaids.org/ pub/BaseDocument/2008/20080801_minsterdeclaration_en.pdf>. En anglais. Également accessible en espagnol sur: <http://data.unaids.org/pub/ BaseDocument/2008/20080801_minsterdeclaration_es.pdf>.

Les Principes de Jogjakarta. Ce document concerne l'application des droits humains aux questions d'orientation sexuelle et d'identité de genre. <www. yogyakartaprinciples.org/principles_fr.htm>. En français. Également disponible en anglais, en arabe, en chinois, en espagnol et en russe.

\section{Principes directeurs internationaux sur l'éducation sexuelle. Une approche} factuelle à l'intention des établissements scolaires, des enseignants et des professionnels de l'éducation à la santé, UNESCO, mai 2010. Non pas un programme scolaire, ce document se concentre sur l'explication de ce que représente l'éducation à la sexualité et de son importance. <http://unesdoc.unesco. org/images/0018/001832/183281f.pdf>. En français. Également disponible en anglais et en espagnol.

Triple Danger Pour Les Jeunes Femmes: Adolescence, Violence Sexuelle et VIH/SIDA, International Women's Health Coalition, 2008. 6 pages. Ce dossier présente des données pertinentes, explique le lien entre la violence sexuelle et la vulnérabilité des jeunes filles au VIH et propose une réponse politique et programmatique. $<w w w$.iwhc.org/index.php?option=com_content\&task=view\&id $=3305 \&$ ltemid=74>. En français. Également disponible en anglais, en espagnol et en portugais. 



\section{UN SEUL PROGRAMME}

